Barbara Seidel

Die Einbindung der Bundesrepublik Deutschland in

die Europäischen Gemeinschaften als Problem des Finanzausgleichs 


\section{Die Einbindung der Bundesrepublik Deutschland in die Europäischen Gemeinschaften als Problem des Finanzausgleichs}

Seit ihrer Gründung haben die Europäischen Gemeinschaften für die einzelnen Mitgliedstaaten immer mehr an Bedeutung gewonnen. Die Einbindung der föderativ aufgebauten Bundesrepublik Deutschland in die Europäischen Gemeinschaften wirft eine Reihe von Finanzausgleichsproblemen auf, die nicht zuletzt durch die zunehmende Verlagerung von Aufgaben auf die Gemeinschaftsebene und den wachsenden Haushalt der Gemeinschaften immer drängender geworden sind. In der vorliegenden Arbeit werden diese Probleme anhand der ökonomischen Kriterien der Föderalismustheorie systematisch identifiziert und Lösungsansätze aufgezeigt. Dabei werden sowohl Aspekte der Beziehungen der Europäischen Gemeinschaften zur Bundesrepublik Deutschland insgesamt als auch die Auswirkungen der europäischen Integration auf den deutschen Finanzausgleich behandelt.

Barbara Seidel wurde 1962 in Stuttgart geboren. Sie studierte Volkswirtschaftslehre an der Eberhard-Karls-Universität Tübingen. Seit ihrem Abschluß als DiplomVolkswirt 1987 arbeitet sie dort als wissenschaftliche Angestellte an der Abteilung Volkswirtschaftslehre, insbesondere Finanzwissenschaft, des Wirtschaftswissenschaftlichen Seminars. Promotion 1992. 
Die Einbindung der Bundesrepublik Deutschland in die Europäischen Gemeinschaften als Problem des Finanzausgleichs 


\section{FINANZWISSENSCHAFTLICHE SCHRIFTEN}

Herausgegeben von den Professoren

Albers, Krause-Junk, Littmann, Oberhauser, Pohmer, Schmidt

Band 50

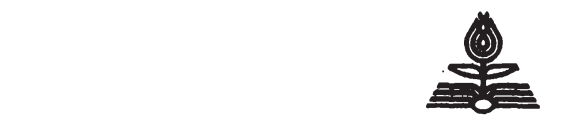

PETER LANG

Frankfurt am Main - Berlin - Bern · New York · Paris - Wien 


\section{Barbara Seidel}

\section{Die Einbindung der Bundesrepublik Deutschland in die Europäischen Gemeinschaften als Problem des Finanzausgleichs}

\section{(3)

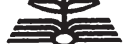 \\ PETER LANG}

Frankfurt am Main - Berlin - Bern · New York - Paris - Wien 
Die Deutsche Blbliothek - CIP-Einheitsaufnahme

Seidel, Barbara:

Die Einbindung der Bundesrepublik Deutschland in die Europäischen Gemeinschaften als Problem des

Finanzausgleichs / Barbara Seidel. - Frankfurt am Main ; Berlin ;

Bern ; New York ; Paris ; Wien : Lang, 1992

(Finanzwissenschaftliche Schriften ; Bd. 50)

Zugl.: Tübingen, Univ., Diss., 1992

ISBN 3-631-45154-7

NE: GT

Open Access: The online version of this publication is published on www.peterlang.com and www.econstor.eu under the international Creative Commons License CC-BY 4.0. Learn more on how you can use and share this work: http://creativecommons. org/licenses/by/4.0.

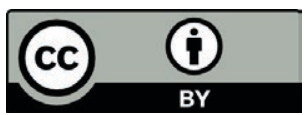

This book is available Open Access thanks to the kind support of ZBW - Leibniz-Informationszentrum Wirtschaft.

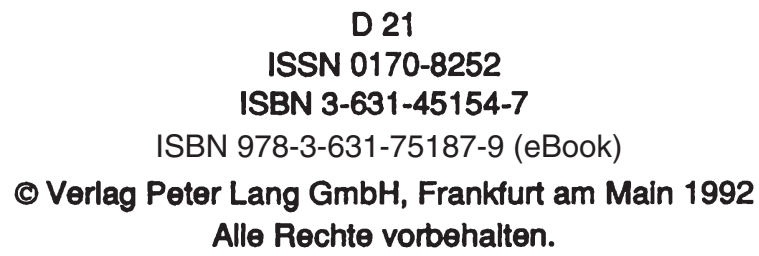

Das Werk einschließlich aller seiner Teile ist urheberrechtlich geschützt. Jede Verwertung außerhalb der engen Grenzen des Urheberrechtsgesetzes ist ohne Zustimmung des Verlages unzulăssig und strafbar. Das gilt insbesondere fūr Vervielfältigungen, Übersetzungen, Mikroverfilmungen und die Einspeicherung und Verarbeitung in elektronischen Systemen.

\section{Printed in Germany $1 \quad 34567$}




\section{Vorwort}

Seit ihrer Gründung haben die Europäischen Gemeinschaften für die Bundesrepublik Deutschland immer mehr an Bedeutung gewonnen. Die Tatsache, daß die Bundesrepublik Deutschland in die Europäischen Gemeinschaften eingebunden ist, wirft eine Reihe von Finanzausgleichsproblemen auf, die nicht zuletzt durch die zunehmende Verlagerung von Aufgaben auf die Gemeinschaftsebene und den wachsenden Haushalt der Gemeinschaften immer drängender geworden sind. Diese Probleme werden in der vorliegenden Arbeit systematisch untersucht. Dabei werden sowohl die Aspekte der Beziehungen der Europäischen Gemeinschaften zur Bundesrepublik Deutschland insgesamt als auch die Auswirkungen der europäischen Integration auf den deutschen Finanzausgleich behandelt.

Die Arbeit wurde im Dezember 1991 abgeschlossen. Neuere Entwicklungen innerhalb der Europäischen Gemeinschaften, insbesondere die im Februar 1992 unterschriebenen Verträge von Maastricht, konnten daher nicht berücksichtigt werden. Das in Kapitel B skizzierte föderative Grundmodell stellt allerdings auch für die Beurteilung dieser Entwicklungen eine Basis dar. Eine erste Durchsicht der beschlossenen Vertragsänderungen legt den Schluß nahe, daß sich die hier beschriebenen Probleme in vielen Bereichen noch verschärfen werden.

Mein besonderer Dank gilt Herrn Professor Dr. Dieter Pohmer, der diese Arbeit anregte und viele wertvolle Hinweise gab. Herzlicher Dank gebührt auch Herrn Professor Dr. Adolf Wagner, der das Korreferat übernahm. Meinen Kollegen, insbesondere Frau Dr. Gisela Jurke, Frau Dr. Françoise Dorison, Herrn Diplom-Volkswirt Jörg Wieland und Herrn Diplom-Volkswirt Thomas Notheis danke ich für manch anregendes Gespräch. Frau Margot Boehme und Frau Magdalena Reisacher haben mir beim Schreiben des Manuskriptes geduldig Hilfe geleistet. Ihnen und allen anderen, die zum Gelingen dieser Arbeit beitrugen, sei nicht zuletzt sehr gedankt.

Tübingen, im März 1992 
Barbara Pflugmann-Hohlstein - 978-3-631-75187-9 Downloaded from PubFactory at 01/11/2019 07:20:05AM

via free access 


\section{Inhaltsverzeichnis}

Vorwort......................................................................... 5

Inhaltsverzeichnis.............................................................. 7

Verzeichnis der Tabellen......................................................... 13

Verzeichnis der Abbildungen.................................................... 17

Verzeichnis der Abkürzungen.................................................... 19

A. Problemstellung ..................................................... 21

B. Elemente einer sinnvollen Gestaltung des Finanzausgleichs .............. 27

I. Vorteile eines föderativen Aufbaus.................................. 27

1. Die Berücksichtigung unterschiedlicher regionaler Präferenzen.. 28

a) Wohlfahrtsgewinne durch eine regional angepaßte

Versorgung mit öffentlichen Leistungen ...................... 28

b) Bessere Information und Kontrolle auf dezentraler Ebene.... 31

2. Wohlfahrtsgewinne durch Mobilität.............................. 32

3. Wohlfahrtsgewinne durch Wettbewerb zwischen den Gebietskörperschaften ............................................... 33

4. Integrationskosten bei zentraler Aufgabenwahrnehmung ......... 33

II. Bedingungen für einen föderativen Aufbau ............................ 38

1. Das Prinzip der fiskalischen Äquivalenz ........................ 38

a) Die Vermeidung von Spillover-Effekten ..................... 39

aa) Allokative Aspekte ....................................... 39

bb) Aspekte der Stabilisierung ............................... 43

cc) Aspekte der Verteilung ................................... 46

b) Grenzen des Prinzips der fiskalischen Äquivalenz ............ 48

2. Die Notwendigkeit einer klaren Zuordnung der Aufgaben ....... 49

3. Das Konnexitätsprinzip .......................................... 50

4. Die Einnahmenautonomie .................................... 52

5. Ansätze zu einer "gerechten" Verteilung der Finanzierungslasten ....................................................... 57

6. Zur Notwendigkeit eines Finanzausgleichs im engeren Sinne..... 59

C. Die Regelungen des Finanzausgleichs in den Europäischen Gemeinschaften und ihre Auswirkungen auf die Bundesrepublik Deutschland.... 61 I. Rechtliche und institutionelle Grundlagen der Europäischen Gemeinschaften ..................................................... 61

1. Die Verträge ................................................... 61 
2. Die Zusammensetzung der Organe der Europäischen Gemeinschaften

3. Der Rechtsetzungsprozeß der Europäischen Gemeinschaften ..... 63

a) Das Zusammenwirken der Organe beim Rechtsetzungsprozeß.

b) Die Instrumente der Rechtsetzung 66

4. Die Durchführung von Rechtsakten der Europäischen Gemeinschaften

II. Die Aufgabenverteilung

1. Die Aufgaben der Europäischen Gemeinschaft für Kohle und Stahl.

2. Die Aufgaben der Europäischen Atomgemeinschaft

3. Die Aufgaben der Europäischen Wirtschaftsgemeinschaft.

a) Errichtung eines Gemeinsamen Marktes .................... 74

b) Gemeinsame Landwirtschaftspolitik ........................ 78

c) Gemeinsame Verkehrspolitik .................................. 79

d) Koordinierung der Wirtschaftspolitik ......................... 81

e) Wirtschaftlicher und sozialer Zusammenhalt ................ 84

f) Forschung und technologische Entwicklung................. 88

g) Umweltpolitik ......................................... 91

4. Die Aufgaben der Gemeinschaften und die nationalen Ebenen Bund, Länder und Gemeinden

a) Die Auswirkungen von Regelungen der Europäischen Gemeinschaften auf Bund, Länder und Gemeinden

aa) Auswirkungen auf den Bund.............................. 93

bb) Auswirkungen auf die Länder ........................... 94

cc) Auswirkungen auf die Gemeinden......................... 95

b) Der Einfluß von Bund und Ländern auf die gemeinschaftliche Rechtsetzung.................................... 96

III. Der Haushalt der Europäischen Gemeinschaften ...................... 102

1. Die Haushaltsinstrumente der Europäischen Gemeinschaften....102

2. Das Haushaltsverfahren für den Gesamthaushalt der Europäischen Gemeinschaften........................................ 104

IV. Die Ausgabenverteilung ............................................... 107

1. Die Ausgaben der Europäischen Gemeinschaften ................. 107

a) Überblick..................................................... 107

b) Die Ausgaben für die Garantie der Agrarmärkte .............110 
c) Die Ausgaben für die Strukturpolitik der Gemeinschaft......112

aa) Die Ausgaben der Strukturfonds .........................112

bb) Sonstige Ausgaben mit struktureller Zweckbestimmung ..............................................114

d) Ausgaben in den Bereichen Energie, Industrie, Technologie und Forschung .

e) Erstattungen an Mitgliedstaaten ...............................116

f) Ausgaben für die Zusammenarbeit mit Entwicklungsländern und Drittländern

2. Die Ausgaben der Europäischen Gemeinschaften und die

Haushalte von Bund und Ländern ...............................118

V. Die Einnahmenverteilung ......................................... 123

1. Das Einnahmensystem der Europäischen Gemeinschaften .......123

a) Die Entwicklung des Einnahmensystems.....................123

aa) Das System der Umlagen und der Finanzbeiträge........123

bb) Der Eigenmittelbeschluß von 1970 ....................... 124

cc) Der Eigenmittelbeschluß von 1985 ...................... 128

b) Das derzeitige Einnahmensystem ............................129

aa) Änderungen bei den "originären" Eigenmitteln durch den Eigenmittelbeschluß von 1988

bb) Mehrwertsteuer-Eigenmittel und Bruttosozialprodukt-Eigenmittel ....................................130

cc) Einführung einer Obergrenze für die Einnahmen insgesamt ............................................. 130

dd) Die Anpassung der Ausgleichsregelung für Großbritannien................................................131

ee) Die Berechnung der Anteile an den Mehrwertsteuer-Eigenmitteln und den BruttosozialproduktEigenmitteln der Mitgliedstaaten für das Haushaltsjahr 1991.............................................133

c) Zur Bedeutung der einzelnen Einnahmenarten.

2. Der Einfluß der Europäischen Gemeinschaften auf die nationalen Einnahmensysteme am Beispiel der Bundesrepublik Deutschland.

a) Einschränkungen der Objekthoheit von Bund und Ländern 
b) Einschränkungen der Ertragshoheit von Bund und Ländern

D. Kritische Würdigung der Finanzausgleichsregelungen in den Europäischen Gemeinschaften unter Berücksichtigung der Situation der Bundesrepublik Deutschland

I. Die mangelnde Berücksichtigung der Vorteile eines föderativen Aufbaus

1. Beachtung des Subsidiaritätsprinzips auf der Gemeinschaftsebene?

2. Die Problematik der unterschiedlichen Präferenzen in den Europäischen Gemeinschaften

a) Grundsätzliches zur Berücksichtigung unterschiedlicher regionaler Präferenzen.

b) Konsensfindungskosten ........................................154

c) Institutionelle Mängel ..................................... 156

3. Überlegungen zu einzelnen Aufgabenbereichen ...................157

a) Die Umweltpolitik .......................................... 158

b) Die Schaffung des Binnenmarktes ............................161

c) Die Technologiepolitik.......................................164

d) Die Regionalpolitik .........................................166

e) Die Agrarpolitik.............................................172

f) Die Stabilisierungspolitik......................................177

II. Das Problem der unklaren Aufgabenzuordnung ..................... 187

1. Die fehlende Transparenz ....................................... 188

2. Zielkonflikte - das Beispiel der Agrarpolitik ...................... 189

3. Koordinierungsprobleme - das Beispiel der Regionalpolitik

III. Probleme der Ausgabenverteilung

1. Die Einteilung in obligatorische und nicht-obligatorische Ausgaben ....

2. Die Dominanz der Agrarausgaben

a) Die grundsätzliche Problematik

b) Ansätze zur Reform der Agrarmarktpolitik.

3. Entlastungen von Bund und Ländern durch die Agrarmarktausgaben der Europäischen Gemeinschaften?. 203

4. Zusätzliche Ausgaben bei Bund und Ländern durch Beschlüsse der Europäischen Gemeinschaften 
5. Die Problematik der Mischfinanzierung - das Beispiel der Strukturfonds ...................................................208

IV. Die Einnahmenautonomie .......................................... 212

1. Die Einnahmenautonomie von Bund und Ländern ..............212

a) Die Anpassung der nationalen Haushalte an den Finanzbedarf der Europäischen Gemeinschaften....................2212

b) Wirkungen auf die nationale Föderalismusstruktur in der Bundesrepublik Deutschland .........................213

c) Nicht-fiskalische Anpassungsprobleme .....................215

2. Die Einnahmenautonomie der Gemeinschaft .....................216

a) Die begrenzten Einnahmenkompetenzen der Europäischen Gemeinschaften.....................................216

aa) Die Einnahmenkompetenzen bei den "originären" Eigenmitteln ............................................216

bb) Die Einnahmenkompetenzen bei den MehrwertsteuerEigenmitteln .........................................2217

cc) Die Einnahmenkompetenzen bei den Bruttosozialprodukt-Eigenmitteln

b) Die Schwierigkeiten bei der Deckung der Gemeinschaftsausgaben ..........................................218

c) Fehlende Rückkopplungseffekte zwischen der Belastung der Bürger mit Abgaben und den Ausgaben der Europäischen Gemeinschaft.......................................220

aa) Die fehlende Merklichkeit .............................220

bb) Sicherung der Rückkopplungseffekte durch eine verstärkte Haushaltsautonomie des Europäischen Parlaments.......................................223

V. Die Diskussion um einen angemessenen Finanzierungsanteil ........225

1. Die Forderung nach "juste retour" ...........................225

2. Die Kappung der Mehrwertsteuer-Bemessungsgrundlage .......235

3. Zur Regressivität der Mehrwertsteuer-Eigenmittel .............240

VI. Zum Finanzausgleich im engeren Sinne ..............................2243

E. Schlußbetrachtung ...................................................251

Literaturverzeichnis ...................................................... 261 
Barbara Pflugmann-Hohlstein - 978-3-631-75187-9 Downloaded from PubFactory at 01/11/2019 07:20:05AM

via free access 
Verzeichnis der Tabellen

Tabelle 1: Die Ziele der Strukturfonds ............................... 86

Tabelle 2: Die Ausgaben des Gesamthaushaltes der Europäischen Gemeinschaften im Vergleich zu den Ausgaben der nationalen Ebenen in der Bundesrepublik Deutschland (in Mrd. DM)

Tabelle 3: $\quad$ Übersicht über die Ausgaben des Gesamthaushaltes der Europäischen Gemeinschaften (in Mio. ECU)

Tabelle 4: Anteil der jährlichen Ausgaben der Abteilung Garantie des Europäischen Ausgleichs- und Garantiefonds für die Landwirtschaft am Gesamtbetrag der Ausgaben (Jährliche Haushaltsausgaben $=100 \%) \ldots \ldots \ldots \ldots . . . . . . . . . . . .112$

Tabelle 5: Der Anteil der Strukturfonds an den Gesamtausgaben in den Jahren 1975 bis 1991 (in \%)

Tabelle 6: Die Aufteilung der Strukturfondsmittel auf die einzelnen Ziele im Jahr 1989 (in Mio. ECU)

Tabelle 7: Die Verteilung der Finanzbeiträge auf die Mitgliedstaaten im Rahmen des siebten Europäischen Entwicklungsfonds (EEF)

Tabelle 8: Ausgaben der Strukturfonds für das Ziel 2, die im Zeitraum 1.1.1989 bis 31.12.1991 in die Bundesrepublik Deutschland fließen (in Mio. ECU)

Tabelle 9: Ausgaben der Strukturfonds für das Ziel 5b, die im Zeitraum1989 bis 1993 in die Bundesrepublik Deutschland (alter Gebietsstand) fließen (in Mio. ECU)...

Tabelle 10: Die Aufbringungsschlüssel des Finanzsystems vor 1970 (in \%)

Tabelle 11: Zur Berechnung des Referenzbetrages

Tabelle 12: Die Finanzierung des Korrekturmechanismus für Großbritannien im Jahre 1991

Tabelle 13: Ermittlung der Mehrwertsteuer(MWSt)-Eigenmittel im Jahre 1991 (in Mio. ECU)

Tabelle 14: Die Bruttosozialprodukt-Eigenmittel 1991 (in Mio. ECU)

Tabelle 15: Die Einnahmen des Gemeinschaftshaushalts nach Einnahmearten in \% der Gesamteinnahmen in den Jahren $1978-1990$ 
Tabelle 16: Die Mehrwertsteuersätze in den Mitgliedstaaten der Europäischen Gemeinschaften im Jahre 1991

Tabelle 17: Übersicht der speziellen Verbrauchsteuern, die nach den Vorschlägen der Kommission aufgehoben oder neu eingeführt werden müßten

Tabelle 18: Verteilung des Umsatzsteueraufkommens in der Bundesrepublik Deutschland im Jahre 1990 148

Tabelle 19: Der Selbstversorgungsgrad bei ausgewählten landwirtschaftlichen Erzeugnissen in den Mitgliedstaaten $1989 / 90$

Tabelle 20: Die Inflationsraten in den Mitgliedsländern der Europäischen Gemeinschaften (in \%)

Tabelle 21: Öffentliche Defizite/Überschüsse in den Mitgliedstaaten in \% des Bruttoinlandsprodukts/Bruttosozialprodukts

Tabelle 22: Staatsschulden der Mitgliedstaaten der Europäischen

Gemeinschaften in \% des Bruttoinlandsprodukts/ Bruttosozialprodukts

Tabelle 23: Erstattungen für Marktordnungsausgaben und nationale Agrarausgaben in der Bundesrepublik Deutschland in den Jahren 1970 bis 1990 (in Mio. DM) .............205

Tabelle 24: Aufgliederung der EFRE-Mittel auf die Länder zwischen 1975 und 1988

Tabelle 25: Die Anteile von Bund, Ländern, Gemeinden und

Europäischen Gemeinschaften am deutschen Steueraufkommen.

Tabelle 26: Nettopositionen und Bruttoinlandsprodukt-pro-Kopf in \% des Gemeinschaftsdurchschnitts der Mitgliedstaaten in den Jahren 1981 bis 1984.

Tabelle 27: Finanzierungsanteile und Anteile am Bruttoinlandsprodukt (BIP) der Mitgliedstaaten 1981 bis 1984

Tabelle 28: Die Relation der Anteile der Agrarabschöpfungen und der Zölle der Mitgliedstaaten zum Anteil am Bruttoinlandsprodukt (BIP) der Gemeinschaft in den Jahren 1986 bis 1988

Tabelle 29: Bruttoinlandsprodukt-pro-Kopf in den Jahren 1986

bis 1988 in \% des Gemeinschaftsdurchschnitts

Tabelle 30: Die Auswirkungen des Korrekturmechanismus im Haushalt 1991

Tabelle 31: Die Mehrwertsteuer-Bemessungsgrundlage vor und nach der Kappung 1989 
Tabelle 32: Die Bruttosozialprodukt(BSP)-Eigenmittel vor und nach der Kappung der Mehrwertsteuer(MWSt)-Bemessungsgrundlage für das Haushaltsjahr 1989

Tabelle 33: Belastungs- und Entlastungswirkungen durch die Kappung in den einzelnen Mitgliedstaaten im Haushaltsjahr 1989

Tabelle 34: Verwendung des Bruttoinlandsproduktes in den Mitgliedstaaten im Jahre 1988 in \% des Bruttoinlandsproduktes zu Marktprei

Tabelle 35: Verteilungswirkungen der Ausgaben des Europäischen Ausgleichs- und Garantiefonds für die Landwirtschaft im Jahr 1988

Tabelle 36: Die Verteilungswirkungen der Ausgaben des Europäischen Fonds für regionale Entwicklung und des Europäischen Sozialfonds im Jahre 1988

Tabelle 37: Die Ausgaben der Strukturfonds insgesamt und gesamte zurechenbare Ausgaben im Jahre 1988 
Barbara Pflugmann-Hohlstein - 978-3-631-75187-9 Downloaded from PubFactory at 01/11/2019 07:20:05AM

via free access 
Verzeichnis der Abbildungen

Abbildung 1: Wohlfahrtsverluste durch ein zentralisiertes Angebot an öffentlichen Gütern ........................................... 29

Abbildung 2: Integrationskosten...................................... 34

Abbildung 3: Meinungsfeld für die Bereitstellung zweier Güter A und $\mathrm{B}$ durch die Kollektivmitglieder $\mathrm{R}, \mathrm{S}, \mathrm{T}, \mathrm{X}, \mathrm{Y}, \mathrm{Z} \ldots \ldots . . .35$

Abbildung 4: Indifferenzkurven gleicher Belastung bei einem Abweichen von der Optimalvorstellung ........................... 36

Abbildung 5: Wirkungen negativer Spillover-Effekte...................... 39

Abbildung 6: Wirkungen positiver Spillover-Effekte ..................... 41

Abbildung 7: Die Zuordnung von Einnahmezuständigkeiten auf die europäischen Gemeinschaften (EG) und die Mitgliedstaaten (MS) 
Barbara Pflugmann-Hohlstein - 978-3-631-75187-9 Downloaded from PubFactory at 01/11/2019 07:20:05AM

via free access 


\section{Verzeichnis der Abkürzungen}

$$
\begin{array}{ll}
\text { BIP } & =\text { Bruttoinlandsprodukt } \\
\text { BSP } & =\text { Bruttosozialprodukt } \\
\text { EAG } & =\text { Europäische Atomgemeinschaft } \\
\text { EAGFL } & =\text { Europäischer Ausgleichs- und Garantiefonds für } \\
& \text { die Landwirtschaft }
\end{array}
$$

EAGV = Vertrag zur Gründung der Europäischen Atomgemeinschaft

EEA = Einheitliche Europäische Akte

EEF = Europäischer Entwicklungsfonds

EFRE = Europäischer Fonds für regionale Entwicklung

EG = Europäische Gemeinschaften

EGKS = Europäische Gemeinschaft für Kohle und Stahl

EGKSV = Vertrag über die Gründung der Europäischen Gemeinschaft für Kohle und Stahl

EIB = Europäischen Investitionsbank

ESF = Europäischer Sozialfonds

EWG = Europäische Wirtschaftsgemeinschaft

EWGV = Vertrag zur Gründung der Europäischen Wirtschaftgemeinschaft

MWSt $=$ Mehrwertsteuer

UStG = Umsatzsteuergesetz 
Barbara Pflugmann-Hohlstein - 978-3-631-75187-9 Downloaded from PubFactory at 01/11/2019 07:20:05AM

via free access 


\section{A. PROBLEMSTELLUNG}

Im Rahmen des Finanzausgleichs werden Probleme behandelt, die sich aus der Vielgliedrigkeit des öffentlichen Sektors, vor allem durch die unterschiedlichen Gebietskörperschaften ${ }^{1}$, ergeben. Entsprechende Probleme werden dann besonders virulent, wenn zu den bestehenden Ebenen ${ }^{2}$ eine neue hinzukommt. Die bisherigen Ebenen müssen Aufgaben abtreten, entsprechend müssen die Ausgaben neu geordnet werden und schließlich ist auch die Einnahmenverteilung anzugleichen. Es entsteht eine neue föderative Struktur.

Die europäische Integration stellt aus der Sicht der Bundesrepublik Deutschland einen solchen Vorgang dar. Zu den drei nationalen Ebenen Bund, Länder und Gemeinden ${ }^{3}$ sind die Europäischen Gemeinschaften als vierte ${ }^{4}$, supranationale Ebene hinzugekommen. Diese ist dadurch entstanden, daß die einzelnen Mitgliedstaaten auf einen Teil ihrer nationalen Souveränität zugunsten gemeinschaftlicher Organe verzichteten, so daß die Gemeinschaft bestimmte, im wesentlichen vertraglich geregelte Zuständigkeiten besitzt. ${ }^{5}$ Die Beziehungen zwischen den Europäischen Gemeinschaften und den Mitgliedstaaten lassen sich nach Peffekoven als eine Form des supranationalen Finanzausgleichs bezeichnen. ${ }^{6}$

In der Regel unterscheidet man einen vertikalen und einen horizontalen Finanzausgleich. Betreffen Finanzausgleichsregelungen Gebietskörperschaften gleicher Ebene, so spricht man von horizontalem Finanzausgleich; beziehen sich die Regelungen auf Gebietskörperschaften unterschiedlicher Ebenen, handelt

1 Die Parafisci, die bei der Gliederung des öffentlichen Sektors grundsätzlich auch - als Erscheinungsformen des funktionalen Föderalismus - zu berücksichtigen sind, werden im folgenden aus der Betrachtung ausgeklammert.

2 Mit dem Begriff "Ebene" soll hier nicht unbedingt ein Über- oder Unterordnungsverhältnis verbunden werden.

3 Rechtlich sind die Gemeinden zwar Teile der Länder. Aus ökonomischer Sicht sind sie jedoch selbständig handelnde Glieder des öffentlichen Sektors.

4 Zum Begriff "vierte Ebene" vgl. u. a. Schüler $=$ Die vierte Ebene $=45 \mathrm{ff}$. und Biskup $=$ Die vierte Ebene $=583$.

$5 \quad \mathrm{Vgl}$. Harbrecht $=\mathrm{EG}=67 \mathrm{f}$.

6 "Bestehen ... überstaatliche Finanzhoheiten, auf die Teile der nationalen Souveränitätsrechte übertragen worden sind, so werden die Beziehungen zwischen den einzelnen Staaten und der von ihnen geschaffenen Organisationen als supranationaler Finanzausgleich bezeichnet." Peffekoven $=$ Finanzausgleich $\mathrm{I}=609$. 


\section{Problemstellung}

es sich um einen vertikalen Finanzausgleich. Die Verteilung von Aufgaben, Ausgaben und Einnahmen ${ }^{7}$ zwischen den nationalen Ebenen und den Europäischen Gemeinschaften ist daher vor allem ein Problem des vertikalen Finanzausgleichs. Das schließt nicht aus, daß hinsichtlich der Verteilungswirkungen der Ausgaben oder Einnahmen der Europäischen Gemeinschaften auch horizontale Effekte zwischen den Mitgliedstaaten auftreten. Man spricht dann von einem vertikalen Finanzausgleich mit horizontaler Wirkung.

Da der Aufbau des öffentlichen Sektors innerhalb der Europäischen Gemeinschaften mehrere Ebenen umfaßt, sind beim vertikalen Finanzausgleich zwei Problembereiche zu trennen: der erste betrifft die Beziehungen der Gemeinschaft zum Mitgliedstaat insgesamt; der zweite betrifft dagegen die Rückwirkungen der Gemeinschaftsaktivitäten auf die Regelungen der nationalen Finanzausgleichssysteme. ${ }^{8}$ Der erste Problembereich ist für alle Mitgliedstaaten ähnlich gelagert; der zweite Aspekt dagegen berührt die Mitgliedstaaten unterschiedlich. Er besitzt für föderativ aufgebaute Staaten größere Bedeutung als für zentralistisch organisierte. Im Rahmen der vorliegenden Arbeit werden beide Aspekte berücksichtigt, d. h. es werden nicht nur die Beziehungen der Europäischen Gemeinschaften zur Bundesrepublik Deutschland insgesamt betrachtet, sondern es werden auch die Auswirkungen der europäischen Integration auf den bundesdeutschen Finanzausgleich berücksichtigt. Dabei liegt der Schwerpunkt der Betrachtung vor allem auf dem Verhältnis zwischen Bund und Ländern, doch werden auch die Gemeinden, wo es notwendig erscheint, in der Betrachtung berücksichtigt.

Die für die Länder getroffenen Aussagen beziehen sich zunächst auf die Länder, die bereits vor dem 3. Oktober 1990 zur Bundesrepublik Deutschland gehörten. Sie betreffen in der Regel jedoch auch die neu hinzugekommenen Länder. Soweit dem nicht so ist, wird dies jeweils vermerkt. Grundsätzlich gilt seit der Herstellung der deutschen Einheit das Europäische Gemeinschaftsrecht

7 Hier wird also in Anlehnung an Peffekoven $=$ Finanzausgleich I $=608 \mathrm{ff}$. und Biehl $=$ Finanzausgleich IV $=691 \mathrm{f}$. von einem weitgefaßten Begriff des Finanzausgleichs ausgegangen. In früheren Untersuchungen des Finanzausgleichs der Europäischen Gemeinschaften wurde häufig nur die finanzielle Sphäre betrachtet, d. h. die Aufgabenverteilung wurde nicht berücksichtigt, so $\mathrm{z}$. B. Ott $=$ Verteilungswirkungen $=$. Noch enger gefaßt wird der Begriff von Franzmeyer, Seidel, die unter dem Finanzausgleich der Europäischen Gemeinschaften nur die Ausgleiçhswirkungen zwischen den Mitgliedstaaten verstehen, vgl. Franzmeyer, Seidel =Überstaatlicher Finanzausgleich $=$.

$8 \mathrm{Zu}$ dieser Unterschiedung vgl. auch Nittka $=$ Finanzierungssystem $=166$. 
- und zwar sowohl die Gemeinschaftsverträge selbst als auch die auf der Grundlage der Gemeinschaftsverträge ergangenen Rechtsakte - auch im Beitrittsgebiet. Für einen Teil des Sekundärrechts der Europäischen Gemeinschaften ${ }^{9}$ wird allerdings eine Anpassung an die Bedingungen des Beitrittsgebietes oder eine Übergangsfrist für die Anwendung der Regelungen eingeräumt. ${ }^{10}$ Dies betrifft vor allem die Bereiche Außenwirtschaft (hinsichtlich der Aufrechterhaltung der Lieferbeziehungen zu den RGW-Staaten), Binnenmarkt (Anforderungen hinsichtlich Sicherheit, Gesundheits- und Verbraucherschutz wurden teilweise bis zum 31.12.1992 ausgesetzt), die Agrarpolitik (Anpassung von Produktionsquoten u. ä.), den Umweltschutz (Ausnahmeregelungen für Altanlagen) und das Wettbewerbs- und Beihilferecht (v. a. Beihilfen für die Stahl- und die Werftindustrie).11

Im ersten Teil der Arbeit wird die Frage gestellt, was bei einer sinnvollen Gestaltung des Finanzausgleichs zu beachten ist. Dabei sollen Kriterien zur Identifizierung von Problemen und Lösungsstrategien entwickelt werden, wobei im wesentlichen auf die ökonomischen Kriterien aus der Föderalismustheorie zurückgegriffen wird. Man mag einwenden, daß die Föderalismustheorie vor allem im Hinblick auf föderative Staatswesen, insbesondere Bundesstaaten, entwickelt wurde, während es sich bei den Europäischen Gemeinschaften aus staatsrechtlicher Sicht lediglich um einen Staatenbund handele. Für den Ökonomen liegt jedoch das Wesen des Föderalismus in der Tatsache, daß die politische Willensbildung und Entscheidung auf mehreren Ebenen erfolgt. Er hat der Frage nachzugehen, welche Kompetenzen welcher Ebene zugeordnet werden sollen, damit ein möglichst hohes Wohlfahrtsniveau für die Bürger des betroffenen Gebietes gewährleistet ist. ${ }^{12}$ Genau diese Problematik der Kompetenzzuordnung auf verschiedene Ebenen stellt sich auch bei den Europäischen Gemeinschaften. Grundsätzlich lassen sich die Überlegungen der Föderalismustheorie daher auch auf die Europäischen Gemeinschaften beziehen. Wenn den Kriterien der Föderalismustheorie allerdings Rahmenbedingungen zugrunde liegen, die in den Europäischen Gemeinschaften (noch) nicht gegeben sind,

9 Nach Angaben der Bundesregierung betrifft dies ca. 20\% des abgeleiteten Gemeinschaftsrechtes. Vgl. Presse- und Informationsamt der Bundesregierung $=$ Einbeziehung $=1$.

10 Vgl. dazu Kommission der EG =Deutsche Vereinigung $1990=129 \mathrm{ff}$.

$11 \mathrm{Zu}$ den Übergangsregelungen und Übergangsfristen vgl. auch Kuschel $=$ Einbeziehung $=80 \mathrm{ff}$.

12 Vgl. Hanusch $=$ Staatsaufgaben $=360$. 


\section{Problemstellung}

wie z. B. ein einheitliches Währungs- oder Rechtsgebiet, müssen sie für die Europäischen Gemeinschaften neu durchdacht und auf diese zugeschnitten werden.

Im übrigen stellt die Föderalismustheorie keine abgeschlossene Konzeption dar, die nur noch anzuwenden wäre; sie liefert lediglich einzelne Elemente für ein föderatives Referenzsystem. ${ }^{13}$ Es geht also nicht darum, eine Gesamtkonzeption für einen optimalen Finanzausgleich zu erarbeiten; dies gilt insbesondere für die Aufgabenverteilung. Da einige Argumente für, andere gegen eine Zentralisierung von Kompetenzen sprechen, bleibt es Aufgabe der Politiker, die Kriterien zu gewichten und letztendlich zu entscheiden. Die theoretischen Überlegungen sollen jedoch helfen, die Ursachen bestimmter Mängel des Finanzausgleichssystems der Europäischen Gemeinschaften aufzuzeigen und Ansatzpunkte für Lösungen zu finden.

Im zweiten Teil der Arbeit wird die föderative Struktur, die sich herausgebildet hat, skizziert. Dabei wird einerseits die Verteilung der Aufgaben, Ausgaben und Einnahmen auf die Europäischen Gemeinschaften und die Mitgliedstaaten dargestellt, andererseits werden die Rückwirkungen der europäischen Integration auf die nationalen Ebenen deutlich gemacht. Es kommt hier nicht darauf an, die Aufgaben der Europäischen Gemeinschaften bis ins letzte Detail nachzuzeichnen - dies würde den Rahmen dieser Arbeit bei weitem sprengen -, sondern es sollen die wichtigsten Aufgabenbereiche skizziert und bestimmte Entwicklungstendenzen deutlich gemacht werden. Bei der Untersuchung der Ausgaben und Einnahmen der Europäischen Gemeinschaften stößt man auf eine Reihe von Einzelproblemen, die den Haushalt der Europäischen Gemeinschaften betreffen. Verstöße gegen Haushaltsgrundsätze u. ä. werden jedoch nur insoweit berücksichtigt, als sie konkrete Auswirkungen im Rahmen des Finanzausgleichssystems haben.

Im letzten Teil der Arbeit werden schließlich die Hauptprobleme des Finanzausgleichs in den Europäischen Gemeinschaften herausgearbeitet, wobei die im ersten Teil gewonnenen Erkenntnisse mit den im zweiten Teil dargestellten Finanzausgleichsregelungen konfrontiert werden.

13 Vgl. dazu auch Biehl, Winter $=$ EG-Finanzierung $=26$. 
In der ganzen Arbeit werden Aufgabenverteilung, Ausgabenverteilung und Einnahmenverteilung weitgehend nacheinander behandelt. Allerdings muß schon hier darauf hingewiesen werden, daß zwischen diesen Bereichen Interdependenzen bestehen, auf die immer wieder aufmerksam gemacht wird. 
Barbara Pflugmann-Hohlstein - 978-3-631-75187-9 Downloaded from PubFactory at 01/11/2019 07:20:05AM

via free access 


\section{B. ELEMENTE EINER SINNVOLLEN GESTALTUNG DES FINANZ- AUSGLEICHS}

\section{Vorteile eines föderativen Aufbaus}

Als wichtigstes Prinzip der Aufgabenverteilung in einem föderativen System gilt das Subsidiaritätsprinzip. Dieses Prinzip ist zuerst als wichtiger sozialphilosophischer Grundsatz der katholischen Soziallehre für den Aufbau der gesamten Gesellschaft in Erscheinung getreten. In "Quadragesimo anno", der Enzyklika über die gesellschaftliche Ordnung, heißt es:

"... wie dasjenige, was der Einzelmensch aus eigener Initiative und mit eigenen Kräften leisten kann, ihm nicht entzogen und der Gesellschaftstätigkeit zugewiesen werden darf, so verstößt es gegen die Gerechtigkeit, das, was die kleineren und untergeordneten Gemeinwesen leisten und zum guten Ende führen können, für die weitere und übergeordnete Gemeinschaft in Anspruch zu nehmen; zugleich ist es überaus nachteilig und verwirrt die ganze Gesellschaftsordnung. Jedwede Gesellschaftstätigkeit ist ja ihrem Wesen und Begriff nach subsidiär, sie soll die Glieder des Sozialkörpers unterstützen, darf sie aber niemals zerschlagen oder aufsaugen.

Bei der Interpretation dieses Prinzips unterscheidet O. v. Nell-Breuning eine positive und eine negative Version, die sich jedoch nicht widersprechen, sondern die "beiden Seiten einer Medaille"2 sind. Nach der negativen Version haben der Einzelne oder die Gliedgemeinschaft in dem Umfang, in dem sie sich selbst helfen können, sowohl die Pflicht als auch das Recht dazu; "erst wo ihre Leistungsfähigkeit überfordert wäre, darf die Gemeinschaft helfend beispringen und ihnen die Leistung abnehmen, sie teilweise oder ganz auf sich übernehmen". 3 Die Hilfe der Gesellschaft (Gemeinschaft) darf die Hilfe zur Selbsthilfe der einzelnen und der "kleineren Lebenskreise" nicht beeinträchtigen; vielmehr fordert die positive Version des Subsidiaritätsprinzips: "Was immer die Gesellschaft (Gemeinschaft) tut, soll den Gliedern 'subsidium afferre', Hilfe bringen, Beistand leisten, förderlich sein." 4

1 Quadragesimo anno: Enzyklika über die gesellschaftliche Ordnung vom 31.5.1931, Ziff. 79; zitiert nach Nell-Breuning $=$ Subsidiaritätsprinzip $=826$.

$2 \quad$ Nell-Breuning $=$ Subsidiaritätsprinzip $=827$.

3 Nell-Breuning $=$ Subsidiaritätsprinzip $=827$.

4 Nell-Breuning $=$ Subsidiaritätsprinzip $=826$. 
Das Subsidiaritätsprinzip erlangte zum einen für die Sozialpolitik große Bedeutung ${ }^{5}$, zum anderen ist es auch grundlegend für den Aufbau föderativer Staatswesen. ${ }^{6}$ Dabei wird die Aufgabenkompetenz grundsätzlich bei der untersten staatlichen Ebene vermutet. "Nur wenn diese nicht imstande ist, eine Aufgabe zu übernehmen, oder die höhere Ebene diese Aufgabe besser wahrnehmen kann, ist die Verlagerung nach oben geboten. ${ }^{7}$

Auch der föderative Aufbau innerhalb der Europäischen Gemeinschaften muß anhand dieser Grundregel beurteilt werden. Dazu muß freilich konkretisiert werden, was "imstande sein" und "besser" bedeutet. Zunächst werden daher die Argumente diskutiert, die für einen möglichst hohen Grad an Dezentralisierung sprechen, so daß deutlich wird, warum der Ansatz des Subsidiaritätsprinzips, bei der Aufgabenverteilung zunächst bei der untersten Ebene zu beginnen, sinnvoll ist. Danach werden die Bedingungen erörtert, die gegeben sein müssen, damit die Vorteile eines föderativen Aufbaus wirksam werden. Dabei werden einerseits Grenzen des Subsidiaritätsprinzips deutlich, andererseits werden Anforderungen an die Ausgabenverteilung und die Einnahmenverteilung als Randbedingungen des Subsidiaritätsprinzips diskutiert.

\section{Die Berücksichtigung unterschiedlicher regionaler Präferenzen}

\section{a) Wohlfahrtsgewinne durch eine regional angepaßte Versorgung mit öffentlichen Leistungen}

Hinter dem Subsidiaritätsprinzip verbergen sich vor allem allokative Überlegungen. Eine einheitliche Versorgung mit öffentlichen Leistungen führt bei unterschiedlichen regionalen Präferenzen im Vergleich zu einer regional angepaßten Versorgung zu Wohlfahrtsverlusten. Unterschiede in den Präferenzen können mehrere Ursprünge haben. Einerseits kann man sie auf unterschiedliche Einstellungen zurückführen, die sich durch unterschiedliche Sprachen, kulturelle Traditionen und geschichtliche Erfahrungen begründen lassen. Andererseits können unterschiedliche sozioökonomische Ausgangspunkte, charakterisiert z. B. durch das Sozialprodukt-pro-Kopf oder auch durch verschiedene politische Institutionen, zu einem beträchtlichen Maß an Meinungsver-

$5 \quad$ Vgl. Nell-Breuning $=$ Subsidiaritätsprinzip $=831$.

6 Vgl. dazu u. a. Isensee $=$ Subsidiaritätsprinzip und Verfassungsrecht $=$.

7 Peffekoven $=$ Finanzausgleich $\mathrm{I}=610$. 
schiedenheiten über Fragen führen, die in einer föderativen Gemeinschaft gemeinsam entschieden werden müssen. 8

Abbildung 1: Wohlfahrtsverluste durch ein zentralisiertes Angebot an öffentlichen Gütern

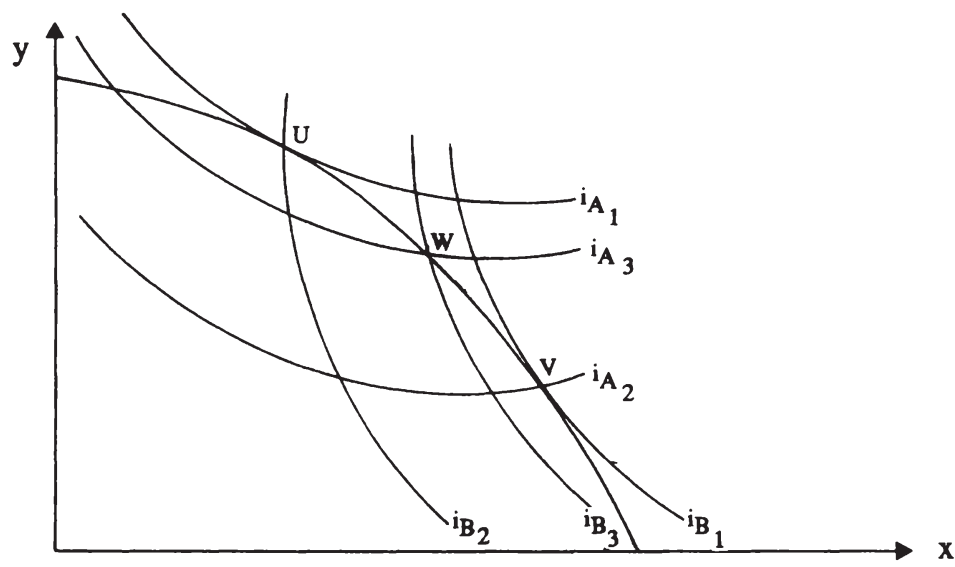

Quelle: Wust $=$ Föderalismus $=23$. Die Originalabbildung wird hier in leicht modifizierter Form wiedergegeben.

An einem einfachen Zwei-Gruppen-zwei-Güter-Modell ${ }^{9}$ lassen sich die Wohlfahrtsgewinne durch eine regional angepaßte Versorgung mit öffentlichen Leistungen verdeutlichen. Dabei soll ein öffentliches Gut y angeboten werden, das jeweils allen Bürgern einer bestimmten Gruppe in gleicher Menge zur Verfügung steht. Die Mitglieder der Gruppe können das Gut gemeinsam und ohne Rivalität nutzen. Die Konsumenten entscheiden sich nur zwischen dem öffentliches Gut y und dem privaten Gut $\mathbf{x}$. Bei der graphischen Darstellung werden der Einfachheit halber nur zwei Gruppen A und B betrachtet. Beide besitzen unterschiedliche gesellschaftliche Wohlfahrtsfunktionen, die sich durch die Indifferenzkurvensysteme $i_{A}$ und $i_{B}$ darstellen lassen.

8 Vgl. Faber, Breyer $=$ Konstitutionelle Aspekte $=215$.

9 Vgl. dazu Wust $=$ Föderalismus $=23 \mathrm{ff}$. 
Läßt man die Bürger über das Angebot der Güter $x$ und $y$, das sie allein in ihrer Gruppe nutzen und finanzieren, selbst entscheiden, so erhält man gruppenspezifische Pareto-Optima in Punkt U und in Punkt V. Wenn eine unabhängige Entscheidung beider Gruppen unzulässig ist und ein einheitliches Güterangebot geschaffen wird, so wird nach dem Mehrheitsprinzip entweder Punkt U oder Punkt V verwirklicht, je nachdem ob Gruppe A oder Gruppe B die Mehrheit besitzt. Die jeweilige Minderheit erleidet eine Nutzeneinbuße. ${ }^{10}$ Wird ein Kompromiß erzielt, so wird ein Punkt zwischen U und V realisiert und beide Bevölkerungsgruppen erreichen nur ein niedrigeres Nutzenniveau. "Eine räumlich einheitliche Versorgung mit dem öffentlichen Gut stellt also mindestens eine Gruppe schlechter." 11

Daß sich die Anpassung des öffentlichen Leistungsangebotes an die Präferenzen der Bürger mit sinkender Gruppengröße verbessern kann, zeigt sich auch an folgenden Überlegungen, die auf Pennock ${ }^{12}$ zurückgehen: Stehen in einer Gruppe die Politik A und die Politik B zur Wahl und ergibt sich eine Mehrheit für Politik A, so wird diese in die Tat umgesetzt. Die Minderheit, die Politik B präferiert hat, erleidet eine Nutzeneinbuße. Spaltet man nun diese Gruppe in zwei Teile auf, die unabhängig voneinander agieren, so wird sich zumindest in einer Teilgruppe eine Mehrheit für die Politik A finden. Entscheidet sich auch die zweite Teilgruppe für die Politik A, so bleibt der Frustrationsgrad der gesamten Gesellschaft gleich. Es ist jedoch auch möglich, daß die zweite Teilgruppe die Politik B präferiert und entsprechend realisiert. Dann steigt insgesamt die Zahl der Mitglieder, die durch die Gruppenentscheidung zufrieden gestellt werden. Allgemein gilt, daß sich mit sinkender Gruppengröße die Frustration der Mitglieder durch Entscheidungen, die ihren Wünschen nicht entsprechen, vermindert. ${ }^{13}$

Insofern verspricht ein dezentrales politisches Entscheidungssystem eine erhöhte ökonomische Effizienz durch eine Angebotskombination öffentlicher Güter, die den Ansprüchen der Konsumenten besser gerecht wird. ${ }^{14}$ Dieses Argument ist vor allem dann bedeutsam, wenn man davon ausgehen kann, daß

10 Oft wird von der "frustrierten Minderheit" gesprochen, so z. B. Forte =Zuordnung ökonomischer Funktionen $=362$.

11 Wust $=$ Föderalismus $=25$.

12 Vgl. Pennock = Disharmony and Frustration $=147 \mathrm{ff}$.

13 Vgl. Tullock $=$ Föderalismus $=29 \mathrm{f}$.

14 Vgl. Peffekoven $=$ Finanzausgleich $\mathrm{I}=611 \mathrm{f}$. 
kleinere Gemeinwesen infolge der Einflüsse der gemeinsamen Umwelt und der kulturellen Tradition homogener sind als größere Gemeinwesen, so daß die "frustrierte Minderheit" geringer ist. ${ }^{15}$ Die Heterogenität der Präferenzen ist in einem Gebilde wie den Europäischen Gemeinschaften, in dem sich Staaten mit unterschiedlicher Geschichte, Entwicklung, Sprache, Kultur und Wirtschaftsstruktur zusammenschließen, weit größer als innerhalb eines Mitgliedstaates. ${ }^{16}$ Daher sind regional angepaßte öffentliche Leistungen in den Europäischen Gemeinschaften noch bedeutsamer als in den Mitgliedstaaten.

\section{b) Bessere Information und Kontrolle auf dezentraler Ebene}

Sollen öffentliche Leistungen "in weitgehender Übereinstimmung mit den Präferenzen der Bürger angeboten werden, so setzt dies die Kenntnis dieser Präferenzen voraus, und es steht $\mathrm{zu}$ vermuten, daß auf den nachgeordneten Ebenen ... diese Bedingung eher erfüllt ist"17. Für die zentrale Ebene gestaltet sich die Beschaffung von Informationen schwieriger und sie gelangt $\mathrm{zu}$ ungenaueren Ergebnissen, da sie mit den Gegebenheiten vor Ort weniger vertraut ist. 18 Bekommt die zentrale Ebene die Informationen von dezentralen Stellen, so ist sie auf deren Zusammenarbeit angewiesen, und es besteht die Gefahr, daß während des Informationsflusses Information verlorengeht. Je länger dabei der Weg ist,

"umso stärker das von der Verwaltung verursachte 'Rauschen' in
den Kommunikationskanälen, umso ausgeprägter die Auswir-
kungen der Präferenzen der Beamten auf die Kollektiventschei-
dung, umso schwerwiegender die Probleme der Informationsüber-
tragung. Diese Kosten steigen . stetig mit wachsender Verwal-
tung; ja es ist nicht auszuschließen, daß sie progressiv ver-
laufen."19

Je näher eine Regierung also dem Gebiet ist, in dem ein Bedarf an öffentlichen Leistungen gedeckt werden soll, desto schneller und genauer kann ihre Verwaltung diese Bedürfnisse ausmachen und entsprechend flexibel kann sie auch auf die Bedürfnisse reagieren. ${ }^{20} \mathrm{Je}$ geringer die Bürgernähe ist, desto weniger

15 Vgl. Forte $=$ Zuordnung ökonomischer Funktionen $=361 \mathrm{f}$.

16 Vgl. dazu auch Biehl, Winter =EG-Finanzierung $=41$.

17 Peffekoven $=$ Finanzausgleich $\mathrm{I}=611$.

18 Vgl. Hanusch $=$ Staatsaufgaben $=363$.

19 Tullock $=$ Föderalismus $=32$.

20 Vgl. Forte $=$ Zuordnung ökonomischer Funktionen $=354$. 
wird die zentrale Ebene bereit sein, unterschiedliche Präferenzen zu berücksichtigen, um so eher wird die Versorgung mit öffentlichen Gütern nivelliert. ${ }^{21}$

Das Leistungsangebot untergeordneter Ebenen kommt den Präferenzen der Bürger auch deshalb näher, weil die Bürger besser kontrollieren können, ob die Leistungen wirklich ihren Bedürfnissen entsprechen. Kleine Verwaltungsapparate können besser durch die Politiker, kleine Regierungsapparate besser durch die Wählerschaft kontrolliert werden. ${ }^{22}$ Geringe Bürgernähe kann zudem zur Folge haben, daß nicht alle Interessen die gleiche Chance haben, gehört zu werden. Gut organisierte Interessenvertretungen haben häufig einen besseren Zugang zum politischen Entscheidungsträger, so daß ihren Wünschen stärker Rechnung getragen wird.

\section{Wohlfahrtsgewinne durch Mobilität}

In einem dezentralen System können Wohlfahrtssteigerungen auch durch die räumliche Mobilität der Bürger erreicht werden. Selbst wenn die Präferenzen stark streuen, und damit einzelne Bürger in einer Region benachteiligt werden, bleibt in einem föderalistischen System der Ausweg, daß Bürger, die ihre Präferenzen in einer Region nicht berücksichtigt sehen, in eine andere "auswandern" können. ${ }^{23}$ Dieses Argument besitzt für die Europäischen Gemeinschaften bisher allerdings noch geringere Bedeutung als für einen Nationalstaat, da die Mobilität zwischen den Mitgliedstaaten geringer ist als innerhalb eines solchen. Obwohl sich die Mobilität mit Fortschritten bei den Freizügigkeits- und Niederlassungsvorschriften ${ }^{24}$ verbessern läßt, bleiben doch zahlreiche Mobilitätshemmnisse im nichtjuristischen Bereich bestehen, wie z. B. Sprachbarrieren.

21 Vgl. Oates $=$ Föderalismusproblem $=20$.

22 Vgl. Forte $=$ Zuordnung ökonomischer Funktionen $=364$.

23 Diese Überlegungen gehen auf Tiebout $=$ Local Expenditures = zurück. Man spricht hier auch von einer "Ábstimmung mit den Füßen".

24 Vgl. Rat der EG =Freizügigkeit $1968=2 \mathrm{ff}$. und Rat der EG = Aufenthaltsrecht $1990=26 \mathrm{f}$. 


\section{Wohlfahrtsgewinne durch Wettbewerb zwischen den Gebietskör- perschaften}

Unter dynamischen Gesichtspunkten lassen dezentrale Entscheidungen erwarten, "daß Innovationen im Angebot kollektiver Dienste häufiger vorkommen"25. Dies ergibt sich aus der Tatsache, daß die dezentralen Entscheidungsträger unter dem Druck des Wettbewerbs stehen. "Sie müssen befürchten, daß die Bürger aus ihrem Gemeinwesen abwandern oder zumindest opponieren, sobald andere Kollektive das gleiche Bedürfnis billiger oder wirksamer befriedigen." 26 Der Wettbewerb zwischen verschiedenen Regionen kann dadurch zu mehr Innovationen führen, sowohl in der Qualität und der Art der öffentlichen Leistung als auch unter administrativen Gesichtspunkten. ${ }^{27}$

\section{Integrationskosten bei zentraler Aufgabenwahrnehmung}

Die oben dargestellten Nutzeneinbußen durch eine zentrale Bereitstellung von öffentlichen Leistungen können aus der Sicht der ökonomischen Verfassungstheorie, die auf Buchanan und Tullock zurückgeht, als Teil der Integrationskosten angesehen werden. Diese entstehen, wenn sich mehrere Kollektivmitglieder ${ }^{28}$ mit unterschiedlichen Interessen zu einer gemeinsamen Politik entschließen. ${ }^{29}$ Für die Europäischen Gemeinschaften ist der Fall, daß in einem Gremium von Vertretern einzelner Gebietskörperschaften über die zentrale Politik entschieden wird, deshalb so interessant, weil genau dies im Ministerrat geschieht.

Aufgrund der unterschiedlichen Interessenlagen ergeben sich für das einzelne Mitglied eines solchen Kollektivs bei einer gemeinschaftlichen Aktivität Kosten. Diese bestehen nicht nur darin, daß den Präferenzen der einzelnen Gruppe dann, wenn sie überstimmt wird, nicht Rechnung getragen wird

25 Wust $=$ Föderalismus $=29$.

26 Wust $=$ Föderalismus $=29 \mathrm{f}$.

27 Vgl. Forte $=$ Zuordnung ökonomischer Funktionen $=365$.

28 Die Kollektivmitglieder sind hier allerdings keine Individuen, sondern Gebietskörperschaften bzw. deren Vertreter.

29 Zur allgemeinen theoretischen Aufarbeitung dieses Problems vgl. Buchanan, Tullock $=$ Calculus of Consent $=$ sowie die Weiterentwicklung durch Kirsch, Theiler $=$ Verallgemeinerung $=$. 
(Expected External Costs) ${ }^{30}$, sondern auch darin, daß jedes Kollektivmitglied versucht, andere Mitglieder zu einer Stimmabgabe in seinem Sinne zu veranlassen (Konsensfindungskosten). Beide Kostenarten gemeinsam stellen die Integrationskosten dar, und diese sind um so höher, je unterschiedlicher die Präferenzen sind. Bei gegebenen Präferenzen ist die Gefahr überstimmt zu werden um so niedriger, je mehr Ja-Stimmen für einen gültigen Beschluß notwendig sind, d. h. die Expected External Costs erreichen bei Einstimmigkeit ihr Minimum. Dagegen sind die Konsensfindungskosten um so höher, je mehr Mitglieder überzeugt werden müssen, d. h. sie erreichen bei Einstimmigkeit ihr Maximum. 31

Abbildung 2: Integrationskosten

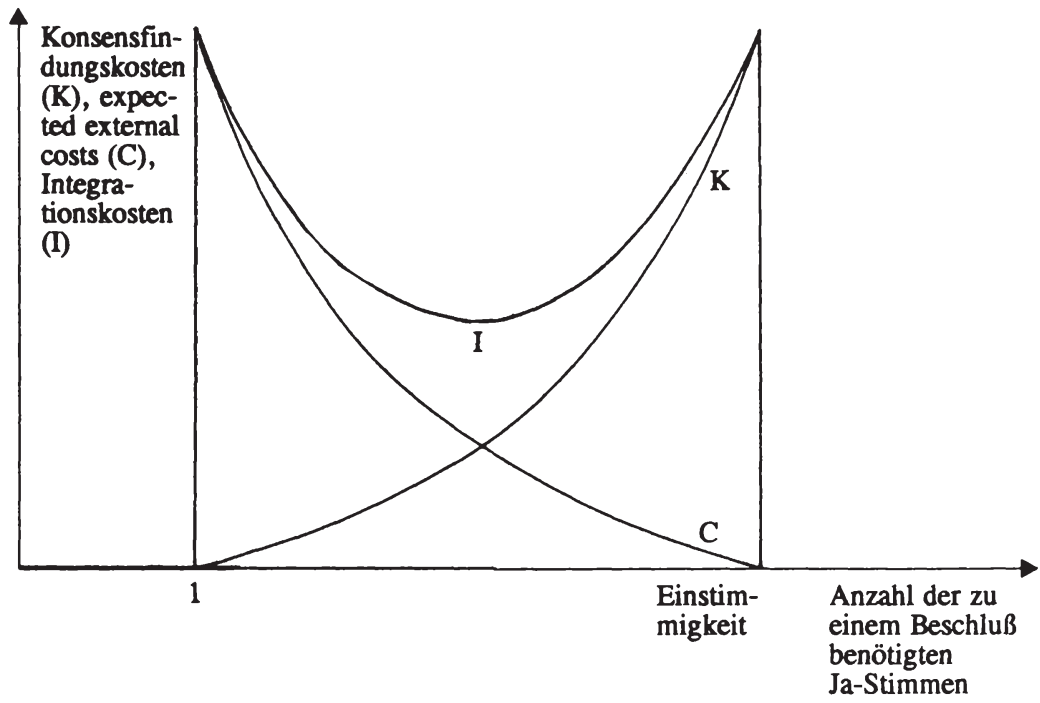

Quelle: Kirsch, Theiler $=$ Verallgemeinerung $=37$. Die Originalabbildung wird hier in leicht modifizierter Form wiedergegeben.

Kirsch und Theiler haben die Konsensfindungskosten weiter untersucht und in drei verschiedene Kategorien eingeteilt. Die erste Kategorie $\left(D_{I}\right)$ umfaßt Kon-

30 Vgl. Buchanan, Tullock $=$ Calculus of Consent $=63$.

31 Vgl. Buchanan, Tullock $=$ Calculus of Consent $=64 f$. 
zessionen auf der Ebene des für das Kollektiv spezifischen Güterangebots. Bei der zweiten Kategorie $\left(D_{\text {II }}\right)$ handelt es sich um Konzessionen in der "Währung" nichtkollektivspezifischer Güter, und die dritte Kategorie ( $\left.D_{\text {III }}\right)$ beinhaltet schließlich Zeit, Nerven, psychologisches Engagement etc. ${ }^{32}$

Da alle drei Kategorien für die Entscheidungsfindung auf Gemeinschaftsebene von Bedeutung sind, sollen sie kurz noch näher betrachtet werden. Dazu werden die unterschiedlichen Interessenlagen der Kollektivmitglieder durch unterschiedliche Positionen im politischen Meinungsfeld dargestellt.

Abbildung 3: Meinungsfeld für die Bereitstellung zweier Güter A und B durch die Kollektivmitglieder R, S, T, X, Y, Z

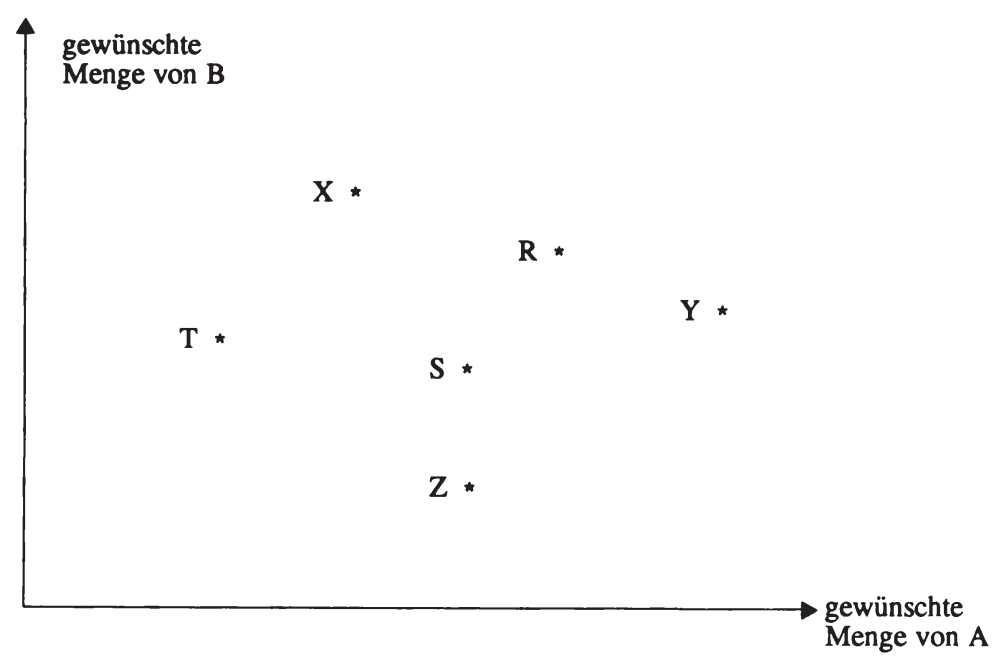

Quelle: Kirsch, Theiler $=$ Verallgemeinerung $=42$. Die Originalabbildung wird hier in leicht modifizierter Form wiedergegeben.

$D_{\mathrm{I}}$ bedeutet konkret, daß das Kollektivmitglied auf die Realisierung des von ihm als optimal angesehenen Leistungsangebots verzichtet und sich dem Standpunkt derer annähert, die es für eine gemeinsame Stimmabgabe gewinnen will. ${ }^{33}$ Den Verzicht, den es dabei leistet, bietet es allen Kollektivmitgliedern

32 Vgl. Kirsch, Theiler $=$ Verallgemeinerung $=43$.

33 Vgl. Kirsch, Theiler $=$ Verallgemeinerung $=43$. 
an, die es erreichen will. Diese werden ihn, je nachdem ob er sich ihren Optimalvorstellungen nähert oder entfernt, positiv oder negativ beurteilen. Ein Abweichen in eine bestimmte Richtung muß dabei nicht immer zu den gleichen Kosten führen wie ein absolut gleich hohes Abweichen in eine andere Richtung. Die Punkte gleicher Belastung durch ein Abweichen von der Optimalvorstellung sind in Abbildung 4 durch Indifferenzkurven dargestellt.

Abbildung 4: Indifferenzkurven gleicher Belastung bei einem Abweichen von der Optimalvorstellung

A

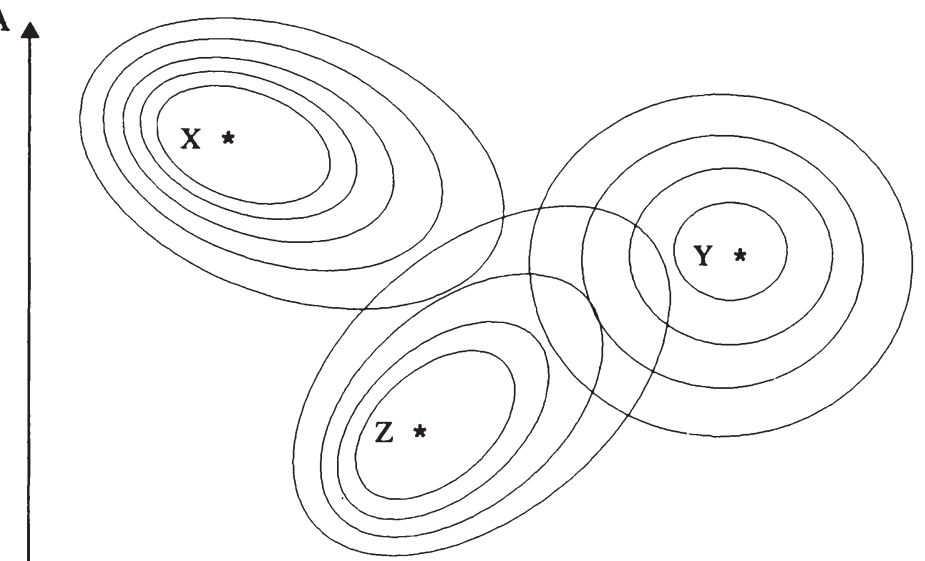

Quelle: Kirsch, Theiler $=$ Verallgemeinerung $=48$. Die Originalabbildung wird hier in leicht modifizierter Form wiedergegeben.

Für die Höhe von $D_{I}$ ist entscheidend, wie weit die zu gewinnenden Kollektivmitglieder entfernt sind, wie eng die Indifferenzkurven auf der Seite zusammenliegen, in deren Richtung das Mitglied seinen Konsenspartner sucht, inwieweit andere von ihrer Position abrücken und wie heterogen die Gruppe möglicher Konsenspartner im politischen Meinungsfeld verteilt ist. ${ }^{34}$

34 Vgl. Kirsch, Theiler $=$ Verallgemeinerung $=47$. 
Bei der zweiten Kategorie $\left(D_{\text {III }}\right)$ handelt es sich ebenfalls um Konzessionen, die jedoch nicht durch kollektivspezifische Güter (im Modell also nicht durch A oder B) erfolgen. ${ }^{35} \mathrm{Um}$ andere Mitglieder dazu zu bewegen, von der Optimalvorstellung abzuweichen, werden Kompensationen angeboten. Diese müssen der Belastung entsprechen, die die Mitglieder zu tragen haben, d. h. hier sind ebenfalls Informationen über die Präferenzen der übrigen Mitglieder notwendig. Auch für die Höhe von $D_{\text {II }}$ ist die Distanz zu den potentiellen Konsensfindungspartnern, die Lage der Indifferenzkurvensysteme und darüber hinaus die Bedeutung des Kompensationsgutes für die Konsensfindungspartner entscheidend. Kompensationszahlungen werden allerdings häufig als anrüchige Praktiken tabuiert ${ }^{36}$. Hier ist das Stichwort Bestechung zu nennen. Aber auch das "log-rolling", bei dem völlig unterschiedliche Probleme zu einem "package-deal" verknüpft werden, ${ }^{37}$ wird oft als "Kuhhandel" disqualifiziert. 38

Auch durch die Konsensfindungskosten der dritten Kategorie $\left(D_{I I I}\right)$, die Zeit, Nerven und psychologisches Engagement beinhaltet, wird versucht, die Kontrahenten zu bewegen, ihren Standpunkt in Richtung auf den eigenen zu verlassen. Dabei spielt wiederum die Distanz zu anderen Kollektivmitgliedern, aber auch die Autorität, die das Kollektivmitglied genießt und die Rigidität der Optimalvorstellung eine Rolle. ${ }^{39}$

Eine gemeinschaftliche Aktivität wird immer nur dann zustande kommen, wenn die Vorteile einer Integration die Nachteile in Form von Integrationskosten übersteigen.

35 Vgl. Kirsch, Theiler $=$ Verallgemeinerung $=47 \mathrm{f}$.

36 Vgl. Kirsch, Theiler $=$ Verallgemeinerung $=49$.

37 Vgl. dazu auch Faber, Breyer $=$ Konstitutionelle Aspekte $=218$.

38 Vgl. Kirsch, Theiler $=$ Verallgemeinerung $=49$.

39 Vgl. Kirsch, Theiler $=$ Verallgemeinerung $=51$. 


\section{Bedingungen für einen föderativen Aufbau}

Die dezentrale Wahrnehmung von Aufgaben hat wichtige Vorteile aufzuweisen. Dies gilt jedoch nur unter bestimmten Voraussetzungen. Die Untersuchung dieser Voraussetzungen zeigt einerseits die Grenzen des Subsidiaritätsprinzips auf, andererseits werden Bedingungen für die konkrete Ausgestaltung des Finanzausgleichs bei der Ausgabenverteilung und der Einnahmenverteilung deutlich.

\section{Das Prinzip der fiskalischen Äquivalenz}

Als erste wichtige Bedingung muß garantiert sein, daß die Grenzen einer Gebietskörperschaft mit der Reichweite der von ihr angebotenen öffentlichen Leistungen (möglichst) übereinstimmen, so daß sichergestellt werden kann, daß jene, die aus den öffentlichen Leistungen einen Nutzen ziehen, auch die sind, die dafür bezahlen (Prinzip der fiskalischen Äquivalenz). Wird dieses Prinzip verletzt, d. h. treten externe Effekte zwischen den Gebietskörperschaften auf, so kommt es zu suboptimalen Lösungen, denn die staatliche Aktivität entfaltet sich in falscher Höhe und Struktur. 1

Externalitäten ergeben sich in Situationen, "in denen die Nutzen des Konsums nicht begrenzt und nicht einem bestimmten Konsumenten zugerechnet werden können, oder Situationen, in denen die ökonomische Aktivität zu Sozialkosten führt, die nicht von dem Produzenten oder Konsumenten bezahlt werden müssen, der sie verursacht"2. Diese zunächst auf Individuen bezogenen Überlegungen kann man auch auf politische Einheiten übertragen: Auch eine Gebietskörperschaft kann öffentliche Leistungen bereitstellen, deren Kostenund/oder Nutzeneffekte über ihre Grenzen hinausgehen. Solche grenzüberschreitenden Effekte werden im folgenden - wie allgemein üblich - als Spillover-Effekte bezeichnet.

$1 \quad$ Vgl. Olson $=$ Fiscal Equivalence $=479 \mathrm{ff}$., hier insbesondere 483.

2 Musgrave, Musgrave, Kullmer $=$ Öffentliche Finanzen $1=54$. 


\section{a) Die Vermeidung von Spillover-Effekten}

\section{aa) Allokative Aspekte}

Die Auswirkungen von negativen Spillover-Effekten werden in Abbildung 5 deutlich. Es wird der Fall dargestellt, daß Land A durch die Bereitstellung des öffentlichen Gutes y in Land B externe Kosten verursacht.

Abbildung 5: Wirkungen negativer Spillover-Effekte

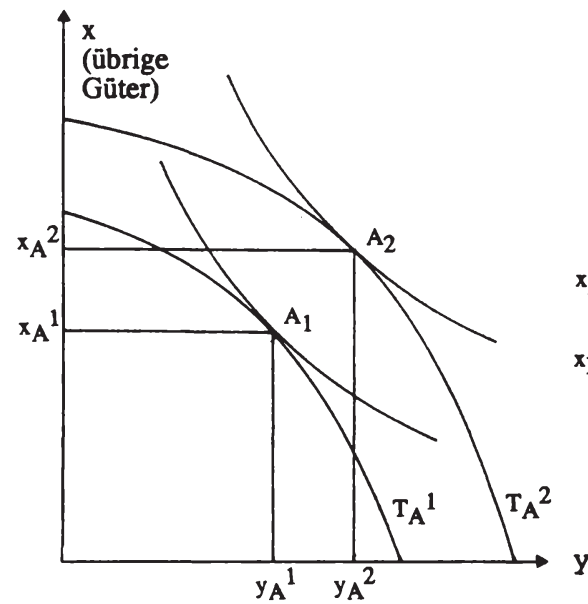

Land $A$

(Verursacher)

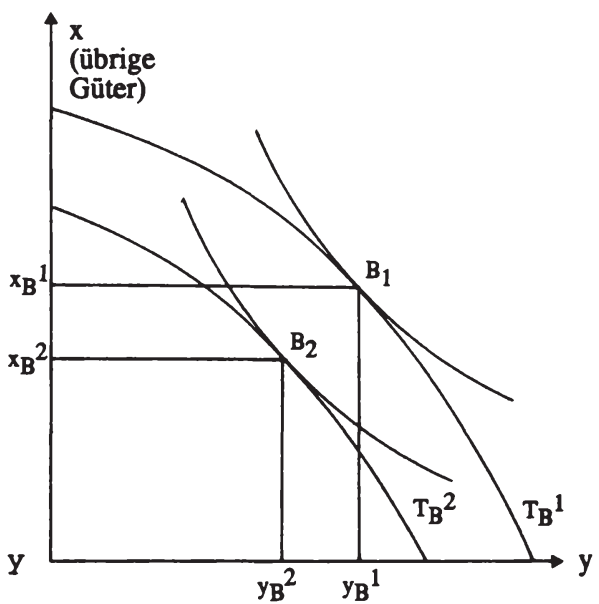

Land $B$

(Belasteter)

$\mathrm{T}_{1}^{1}=$ Transformationskurven in der Ausgangssituation

$\mathrm{T}^{2}=$ Transformationskurven nach dem Auftreten der Spillover-Effekte

$\mathbf{A}_{1}=$ Ausgangssituation in Land $\mathbf{A}$

$A_{2}=$ Situation in Land A nach dem Auftreten von Spillover-Effekten

$\mathrm{B}_{1}=$ Ausgangssituation in Land $\mathrm{B}$

$B_{2}=$ Situation in Land B nach dem Auftreten von Spillover-Effekten

Quelle: Breton $=$ Stabilität des Föderalismus $=133$. Die Originalabbildung wird hier in leicht modifizierter Form wiedergegeben.

Müßte Land A die gesamten Kosten für die Bereitstellung des Gutes y selbst tragen, würde es die Menge $y_{A}^{1}$ bereitstellen. Der Punkt $A_{1}$ wäre pareto-op- 
timal. $^{3}$ Dementsprechend würde Land $\mathrm{B}$ den Punkt $\mathrm{B}_{1}$ mit den Mengen $\mathrm{y}_{\mathrm{B}}{ }^{1}$ und $\mathrm{x}_{\mathrm{B}}{ }^{1}$ realisieren. Fällt jedoch ein Teil der Kosten für die Bereitstellung von $\mathrm{y}_{A}$ anstatt in Land $\mathrm{A}$ in Land $\mathrm{B}$ an, so kann Land A mit den gegebenen Mitteln mehr produzieren, $d$. $h$. seine Transformationskurve $T_{A}$ verschiebt sich vom Ursprung weg $\left(\mathrm{T}_{\mathrm{A}}{ }^{2}\right)$. Land $\mathrm{B}$ kann dagegen unter Berücksichtigung der negativen Spillover-Effekte, die durch Land A anfallen, mit den gegebenen Mitteln nur noch weniger produzieren. Seine Transformationskurve $T_{B}$ verschiebt sich zum Ursprung hin. Land $A$ realisiert nun Punkt $A_{2}$ und stellt damit mehr $y$ bereit als optimal wäre $\left(y_{A}{ }^{2}>y_{A}{ }^{1}\right)$, so daß man in Land $A$ von einer Überversorgung sprechen kann.

Dagegen stellt Land B nur noch $\mathrm{y}_{B}{ }^{2}\left(\left\langle\mathrm{y}_{B}{ }^{1}\right)\right.$ bereit, d. h. in Land B kommt es im Vergleich zur optimalen Situation zu einer Unterversorgung. ${ }^{4}$ Ob insgesamt der Effekt der Überversorgung oder der Unterversorgung überwiegt, kann von vornherein nicht gesagt werden. ${ }^{5}$ Dies hängt zum einen von den Präferenzen der Länder A und B ab, die natürlich auch unterschiedlich sein können, zum anderen von der Verschiebung der Transaktionskurven. Dabei ist zu beachten, daß die Kostenvermeidung in Land A nicht notwendigerweise betragsmäßig gleich groß wie die Kostensteigerung in Land B sein muß.6

Festzuhalten bleibt, daß es gesamtwirtschaftlich fast immer zu einer Situation der Überversorgung oder der Unterversorgung kommt und eine gesamtwirtschaftlich optimale Bereitstellung nur zufällig, meist jedoch gar nicht erreicht wird. ${ }^{7}$ Darüber hinaus bewirken die Spillover-Effekte einen Realvermögenstransfer von Land A nach Land B. Nimmt man die ursprüngliche Verteilung als gerecht an, so ist dieser Realvermögenstransfer ein verteilungspolitisch unerwünschtes und ungerechtes Phänomen. 8

3 Zu den Bedingungen der Pareto-Optimalität vgl. Musgrave, Musgrave, Kullmer =Öffentliche Finanzen $1=80$.

4 Vgl. Neumann $=$ Föderalismus $=506$.

5 Vgl. Williams $=$ Public Goods $=29$ und Neumann $=$ Föderalismus $=506$.

6 Vgl. dazu Kirsch $=$ Die Wahl $=26 \mathrm{ff}$.

7 Vgl. Neumann $=$ Föderalismus $=506$ und Williams $=$ Public Goods $=29$.

8 Vgl. Kirsch $=$ Ökonomische Theorie $=14$. 
Abbildung 6: Wirkungen positiver Spillover-Effekte

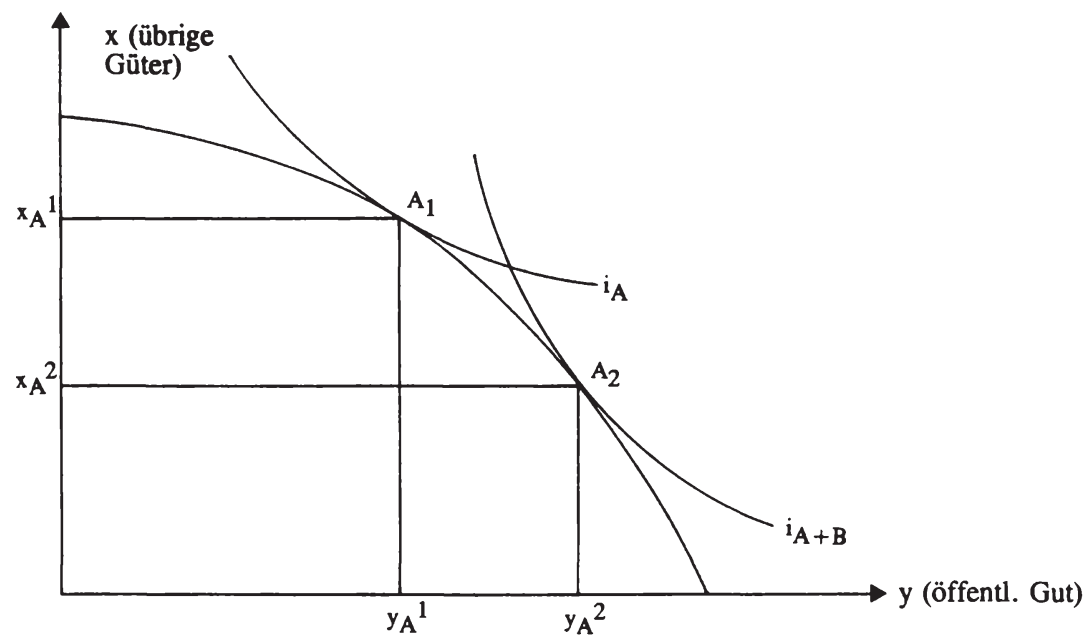

Auch das Auftreten positiver Spillover-Effekte führt zu suboptimalen Lösungen. Abbildung 6 stellt den Fall dar, daß Land A ein öffentliches Gut bereitstellt, dessen Nutzen nicht nur den eigenen Bürgern, sondern auch den Bürgern in Land B zuflieBt. Land A stellt unter ausschließlicher Beachtung der eigenen Präferenzen die Menge $\mathrm{y}_{1}$ des Gutes y bereit. Erfaßt man den zusätzlichen Nutzen, den Gut y auch in Land B stiftet, so erhält man ein neues Indifferenzkurvensystem $I_{A}+B$. Dieses verläuft steiler, da die Grenzrate der Substitution $|-\mathrm{dx} / \mathrm{dy}|$ größer ist. ${ }^{9}$ Unter Berücksichtigung des gesamten Nutzens, den das Gut y stiftet, müßte unter Optimalbedingungen der Punkt $\mathrm{A}_{2}$ realisiert werden. Die optimale Menge des Gutes y ist damit größer als die von Land A unter ausschließlicher Beachtung des eigenen Nutzens bereitgestellte Menge. Die positiven Spillovers bedingen insofern eine Unterversorgungssituation. ${ }^{10}$ Daneben kommt es auch hier zu einer Verschiebung von Realeinkommen, und zwar von Land A nach Land B. ${ }^{11}$

9 Insgesamt wird Gut y jetzt ein größerer Nutzen beigemessen als durch Land A alleine, während Gut $x$ immer noch derselbe Nutzen beigemessen wird. Für die Aufgabe einer kleinen Menge von Gut $y$ werden daher A und B zusammen mehr von Gut $x$ verlangen als Land A alleine.

$10 \mathrm{Vgl}$. Oates $=$ Federal System $=51$.

11 Vgl. Olson, Zeckhauser $=$ Theory of Alliances $=269 \mathrm{f}$. 
Um Spillover-Effekte zu korrigieren, können zunächst Verhandlungen auf horizontaler Ebene geführt werden. Dies ist besonders dann sinnvoll, wenn die Spillover-Effekte nicht nur in einer Richtung auftreten (einseitige Spillovers), sondern die Gebietskörperschaften, von denen Spillover-Effekte ausgehen, ihrerseits von Spillovers anderer Gebietskörperschaften betroffen sind (reziproke Spillovers). ${ }^{12}$ Hier ist das Interesse aller Beteiligten an einer gemeinsamen Lösung recht hoch. Allerdings darf die Zahl der vorhandenen Gebietskörperschaften nicht zu groß sein, denn sonst wächst die Gefahr, daß sich einzelne Gebietskörperschaften als Trittbrettfahrer verhalten, d. h. sie versuchen, sich den Kosten der gemeinsamen Aktivität zu entziehen, wohl wissend, daß sie vom Nutzen nicht ausgeschlossen werden können.

Bei einseitigen Spillover-Effekten ist eine Übereinkunft durch Verhandlungen dann möglich, wenn der Grenzschaden des Betroffenen größer ist als der Grenzgewinn des Verursachers aus der die Belastung hervorrufenden Aktivität. ${ }^{13}$ Eine effizientere Lösung wird hier jedoch auch nur unter sehr restriktiven Bedingungen erreicht: ${ }^{14}$ Die Zahl der Beteiligten darf nicht zu groß sein und Trittbrettfahrer-Verhalten ist auszuschließen. Informations-, Verhandlungs- und Kontrollkosten dürfen nicht anfallen.

Auch wenn diese Voraussetzungen gegeben sind, so daß eine effiziente Lösung erreicht werden könnte, gerät eine solche "freiwillige" Internalisierung durch Verhandlungen möglicherweise mit Restriktionen, die sich aus den Gerechtigkeitsvorstellungen einer Gesellschaft ergeben, in Konflikt. ${ }^{15}$ Solchen Vorstellungen kann die Tatsache, daß der Geschädigte dem Verursacher Zahlungen für die Reduzierung des schädigenden Verhaltens zahlt, widersprechen. Eine Internalisierung der Spillover-Effekte sollte nach diesen Vorstellungen vielmehr dadurch erfolgen, daß der Begünstigte dem Benachteiligten entsprechende Ausgleichszahlungen gewährt. Für beide Seiten wäre damit der Status quo ante wiederhergestellt. ${ }^{16}$ Würde dies auf freiwilliger Basis funktionieren, wäre es unnötig, eine höhere Ebene heranzuziehen. In der Regel sind Kompensa-

12 Vgl. zur Trennung von einseitigen und reziproken Externalitäten Brümmerhoff $=$ Finanzwissenschaft $=63$.

13 Vgl. zu dieser Verhandlungslösung grundlegend Coase $=$ Social Costs $=201 \mathrm{ff}$.

14 Vgl. u. a. Jaeger $=$ Coase-Theorem $=211 \mathrm{ff}$. und Endres $=$ Coase-Kontroverse $=$ $637 \mathrm{ff}$.

15 Vgl. Endres $=$ Coase-Kontroverse $=648$.

16 Vgl. Kirsch $=$ Ökonomische Theorie $=14$. 
tionszahlungen begünstigter Gebietskörperschaften jedoch nicht zu erwarten, "solange keine übergeordnete Entscheidungsebene sie erzwingt"17. Außerdem erfordert die unterschiedliche Einschätzung der anfallenden Kosten oder Nutzen durch beide Seiten ${ }^{18}$ oftmals eine Schlichtung durch übergeordnete Instanzen. "Eine der wichtigsten Funktionen höherer Regierungseinheiten besteht im Fiskal-Föderalismus darin, bei solchen Spillovers den Schiedsspruch zu fällen."19 Auch wenn grundsätzlich die Verhandlungen zwischen den untergeordneten Ebenen geführt werden können, ist es doch sinnvoll, auf übergeordneter Ebene die Rahmenbedingungen für die Verhandlungen festzulegen und die Zahlungen der Kompensationen zu überwachen.

Wenn zu viele Gebietskörperschaften an den Verhandlungen beteiligt sind, so daß ein Trittbrettfahrer-Verhalten nicht ausgeschlossen werden kann, oder wenn die Informations-, Verhandlungs- und Kontrollkosten sehr hoch sind, ist es letztlich sinnvoll, die Entscheidung auf die zentrale Ebene zu verlagern. Insofern kann das Auftreten von Spillover-Effekten zwischen einzelnen dezentralen Gebietskörperschaften Argumente für eine Zentralisierung der Bereitstellung öffentlicher Leistungen liefern.

Für die Aufgabenverteilung in den Europäischen Gemeinschaften bedeutet dies, daß öffentliche Leistungen mit Wirkungen, die (deutlich) über die Grenzen des einzelnen Mitgliedstaates hinausgehen und bei denen bilaterale Verhandlungen zu keinem Ergebnis führen, sinnvollerweise auf der Gemeinschaftsebene erbracht werden sollten.

\section{bb) Aspekte der Stabilisierung}

In der Literatur der Föderalismustheorie wird überwiegend die Meinung vertreten, die zentrale Ebene solle die Hauptverantwortung für die makroökonomische Stabilisierungsfunktion übernehmen. ${ }^{20}$ Die Ziele Preisniveaustabilität und hoher Beschäftigungsstand sind innerhalb eines Wirtschafts- und Währungsraums nicht auf bestimmte Regionen beschränkbar; von den entsprechenden stabilitätspolitischen Maßnahmen einer Region profitieren auch alle

17 Hanusch $=$ Staatsaufgaben $=362$.

18 Vgl. dazu v. a. Kirsch $=$ Die Wahl $=26 \mathrm{ff}$.

19 Tiebout $=$ Fiskalische Dezentralisierung $=49$.

20 Vgl. u. a. Oates $=$ Finanzföderalismus $=297$. 
anderen Regionen. Gelingt es einer Region, die Preise zu stabilisieren, so kommen nicht nur ihre eigenen Einwohner, die (z. B. durch den Verzicht auf zusätzliche öffentliche Leistungen) die Kosten zu tragen haben, in den Genuß der stabilen Preise, sondern auch die Einwohner anderer Regionen, die nun die Güter aus der sich stabilitätsgerecht verhaltenden Region billiger einkaufen können. Nutzen- und Kostenkreise klaffen hier deutlich auseinander; es treten Spillover-Effekte auf. ${ }^{21}$ Eine erfolgreiche Beschäftigungspolitik erhöht das Volkseinkommen der betreffenden Region, dies fördert auch die Nachfrage nach Produkten der anderen Regionen, so daß die Beschäftigung dort ebenfalls tendenziell steigt. Je enger die wirtschaftlichen Verflechtungen zwischen den einzelnen Regionen sind, desto größer sind bei der Stabilitätspolitik die Spillover-Effekte.

Obwohl stabilitätspolitische Maßnahmen im Interesse aller Regionen liegen, sind dezentrale Lösungen nicht erfolgreich, weil aufgrund der Spillover-Effekte jede Region versucht, von der Politik der anderen zu profitieren. Sie hat wenig Anreiz, eigene Maßnahmen zu ergreifen; denn sie fragt sich, weshalb sie allein die daraus entstandenen Nachteile tragen soll, wenn auch andere von den Maßnahmen profitieren. Eigene Maßnahmen sind für die Region wenig effizient, da die Wirkungen räumlich gesehen versickern. ${ }^{22}$

Die Spillover-Effekte treten sowohl bei geldpolitischen als auch bei finanzpolitischen Maßnahmen zur Stabilisierung auf. Es muß eine zentrale Institution vorhanden sein, die das Geldmengenangebot kontrolliert. Wären dezentrale Ebenen zur Geldschöpfung befugt, könnten sie sich mit Hilfe der Druckerpresse Geld für den Kauf realer Güter von benachbarten Gebietskörperschaften beschaffen. Es würde dann im Interesse jeder dezentralen Gebietskörperschaft liegen, ihre Ausgaben eher durch Geldschöpfung zu finanzieren als eigene Mitglieder mit Steuern zu belasten. Das Ergebnis wäre eine wilde Inflation. ${ }^{23}$ Würde sich eine einzelne Gebietskörperschaft geldmengenpolitisch stabilitätsgerecht verhalten, so hätte sie als einzige die Kosten dieser Politik zu tragen, während die umliegenden Gebietskörperschaften auch von den über die wirtschaftlichen Interdependenzen vermittelten Nutzen profitieren würden. Darüber hinaus ist die Wirkung geldpolitischer Maßnah-

21 Vgl. Frey $=$ Föderalismus und Zentralismus $=78$.

22 Vgl. Frey $=$ Föderalismus und Zentralismus $=79$.

23 Vgl. Oates $=$ Föderalismusproblem $=16$. 
men in einer dezentralen Gebietskörperschaft gering, wenn sie zu einem großen Teil von den negativen externen Effekten als Folge des destabilisierenden Verhaltens der umliegenden Gebietskörperschaften kompensiert werden würde. ${ }^{24}$ In welchem Ausmaß entsprechende Wirkungen zu Tage treten, hängt vor allem davon $a b$, wie groß die dezentrale Gebietskörperschaft ist und wie stark sie mit anderen Gebietskörperschaften verflochten ist.

Stehen den dezentralen Gebietskörperschaften monetäre Instrumente nicht zur Verfügung, so bleiben ihnen noch finanzpolitische Instrumente. Doch hier sind die Grenzen für eine aktive, antizyklische Fiskalpolitik ebenfalls sehr eng gezogen: Aufgrund der hohen Importneigung ist der Ausgabenmultiplikator sehr klein. ${ }^{25}$ Damit fallen auch die Wirkungen fiskalpolitischer Stabilisierungsmaßnahmen der dezentralen Gebietskörperschaften als Folge von Spillover-Effekten in der Regel bescheiden aus. ${ }^{26}$

Auch wenn man davon ausgeht, daß eine antizyklische Fiskalpolitik nur dann eingesetzt wird, wenn ein sich selbst verstärkender Abschwung droht, und daß ansonsten eine stetige Ausgabenpolitik betrieben werden soll, bei der die öffentliche Verschuldung innerhalb bestimmter Grenzen zu bleiben hat, muß die Stabilitätspolitik grundsätzlich zentral wahrgenommen werden. Zumindest müssen einige Grundsätze über die Durchführung stabilitätspolitischer Maßnahmen zentral aufgestellt werden.

Hohe Defizite einer Region führen zu einem Zinsanstieg. Dieser bedingt bei freier Kapitalmobilität, die ja in einem einheitlichen Wirtschafts- und Währungsraum gegeben ist, Kapitalimporte. Dadurch werden in den anderen Regionen Investitionen verdrängt. Die Ersparnisse der Bürger dieser Regionen werden zur Finanzierung fremder Haushaltsdefizite beansprucht. Die stabilitätspolitischen Bemühungen in diesen Regionen werden durch die importierte Inflation erschwert. Zwar könnte man einwenden, daß durch die Zinslast der Handlungsspielraum der sich verschuldenden Region sinkt und daß insofern ein Zwang zur Konsolidierung entsteht. Durch die Kapitalimporte verteilen sich die Zinswirkungen allerdings auch auf die anderen Regionen. Dies gilt vor allem dann, wenn sich auch für das verbleibende Bonitätsrisiko Sonderbe-

24 Vgl. Theiler $=$ Föderalismus $=59$.

25 Vgl. Oates $=$ Föderalismusproblem $=16$ und Forte $=$ Zuordnung ökonomischer Funktionen $=367 \mathrm{f}$.

26 Vgl. Theiler $=$ Föderalismus $=59$. 
dingungen ergeben, da der Markt davon ausgeht, daß die Region im Ernstfall nicht sich selbst überlassen bleibt, sondern von den übrigen Regionen gestützt wird. Ob daher der Konsolidierungsdruck rechtzeitig und stark genug einsetzt, um haushaltspolitisches Fehlverhalten zu sanktionieren, ist fraglich. Um die negativen Effekte zu verhindern, kann es daher sinnvoll sein, Grenzen für die Verschuldung der Gebietskörperschaften festzulegen.

W.-D. Füchsel faßt die hier aufgeführten Argumente in dem Fazit zusammen: "Da für stabilitätspolitische Maßnahmen innerhalb eines gemeinsamen Wirtschafts- und Währungsraumes das Ausschlußprinzip nicht gilt, ... muß die stabilitätspolitische Aufgabenkompetenz grundsätzlich zentral wahrgenommen werden. "27 Diese Aussage macht deutlich, daß ein wesentliches Kriterium für die stabilitätspolitische Aufgabenverteilung die Ausschlußmöglichkeit ist, die je nach dem Integrationsstand eines Wirtschafts- und Währungsraumes unterschiedlich stark ausgeprägt sein kann.

\section{cc) Aspekte der Verteilung}

Die Distributionsfunktion muß nach der überwiegenden Meinung der Literatur zum Finanzausgleich zentral wahrgenommen werden. ${ }^{28}$ Der Möglichkeit einer dezentralen Umverteilung werden durch das distributionspolitische Verhalten der übrigen dezentralen Gebietskörperschaften und durch die Mobilität der einzelnen Wirtschaftssubjekte enge Grenzen gesetzt. Wird in einer dezentralen Gebietskörperschaft ein sehr progressives Umverteilungssystem errichtet, so würden - bei ausreichender Mobilität - die Empfänger höherer Einkommen abwandern und ärmere Bürger zuwandern. Das Ergebnis wäre zwar in dieser Gebietskörperschaft eine ausgeglichenere Einkommensverteilung, doch das Einkommensniveau würde sinken. ${ }^{29}$ Die Mobilität der Bürger führt hier zu Externalitäten der distributionspolitischen Entscheidungen dezentraler Gebietskörperschaften. Je geringer die Mobilität allerdings ist, desto eher eröffnen sich dezentralen Gebietskörperschaften eigene Umverteilungsspielräume. Da in den Europäischen Gemeinschaften die Mobilität geringer ist als innerhalb der Mitgliedstaaten, verbleibt diesen noch genügend Spielraum für eine personelle Umverteilung auf nationaler Ebene.

27 Füchsel $=$ Gemeinschaftsaufgaben $=179$.

28 Vgl. u. a. Oates =Finanzföderalismus $=308$ und die dort angegebene Literatur.

29 Vgl. Oates $=$ Föderalismusproblem $=17 \mathrm{f}$. 
Es stellt sich außerdem die Frage, ob die Mobilität gleichermaßen von der Höhe des öffentlichen Leistungsstandards und dem Grad an steuerlicher Belastung verursacht wird. Unter der Annahme, daß die Bürger erst abwandern, wenn die Steuerbelastung erheblich differiert, verbleiben den dezentralen $\mathrm{Ge}$ bietskörperschaften zumindest auf der Ausgabenseite eigenständige Umverteilungsspielräume. ${ }^{30}$

Betrachtet man schließlich noch das regionale Umverteilungsziel "Förderung benachteiligter Regionen", so fällt zwangsläufig das Nutzen- und das Kostenkollektiv auseinander. Folglich muß diese Zielsetzung grundsätzlich zentral wahrgenommen werden. Je stärker allerdings das Gefühl der Solidarität zwischen den Körperschaften ausgeprägt ist, um so weniger braucht die übergeordnete Gebietskörperschaft hierbei aktiv zu werden. ${ }^{31}$

Insgesamt zeigt das Auftreten von Spillover-Effekten, daß einer Dezentralisierung Grenzen gesetzt sein können. Während es uneingeschränkt sinnvoll ist, Aufgaben dezentral wahrzunehmen, wenn die Präferenzen regional sehr stark streuen und wenn keine Spillover-Effekte vorliegen, ist eine Verlagerung von Aufgaben auf eine "höhere" Ebene dann geboten, wenn Spillover-Effekte auftreten, die nicht durch Verhandlungen ausgeglichen werden können, und wenn keine Präferenzunterschiede vorliegen. Zwischen diesen beiden Extremen liegt ein weiter Zwischenbereich, der sowohl durch Präferenzunterschiede als auch durch Spillover-Effekte gekennzeichnet ist. Die sich dadurch ergebenden Vorteile und Nachteile einer Dezentralisierung müssen abgewogen werden. Dies erfordert eine politische Entscheidung. Soll das Subsidiaritätsprinzip dabei korrekt angewandt werden, muß bei einer gleichen Gewichtung der Vorteile und Nachteile die Aufgabe dezentral wahrgenommen werden. Denn in diesem Fall kann die "höhere" Ebene die Aufgabe eben nicht besser wahrnehmen.

Das Prinzip der fiskalischen Äquivalenz hilft also grundsätzlich die Grenzen des Subsidiaritätsprinzips abzustecken. Allerdings ist es selbst ebenfalls Grenzen unterworfen.

30 Vgl. Wust $=$ Föderalismus $=122 \mathrm{f}$.

31 Vgl. Füchsel $=$ Gemeinschaftsaufgaben $=181$. 


\section{b) Grenzen des Prinzips der fiskalischen Äquivalenz}

Geht man vom Prinzip der fiskalischen Äquivalenz aus, so kann es im Extremfall - wenn der Streubereich von Kosten und Nutzen bei jeder öffentlichen Leistung verschieden ist - so viele Kollektive geben, "wie in einer Gesellschaft Bedürfnisse durch Kollektivgüter befriedigt werden sollen"32. Da ein Individuum aber nur an einer begrenzten Anzahl von Kollektiven aktiv gestaltend und auch kontrollierend mitwirken $\mathbf{k a n n}^{33}$, ist eine durchgängige Aufgliederung nach dem Prinzip der fiskalischen Äquivalenz in der Regel unmöglich. 34 Man muß daher versuchen, die Aufgaben so auf eine bestimmte Anzahl von Ebenen zu verteilen, daß die Spillover-Effekte möglichst klein sind. Es ergeben sich dadurch in der Regel Kosteneinsparungen, weil die für die Bereitstellung eines öffentlichen Gutes zu schaffende Organisation in der Regel schon eine so große Entscheidungskapazität aufweist, daß weitere öffentliche Aufgaben mit geringen Grenzkosten übernommen werden können. Der Kostenkalkül wird in der Realität auch davon beeinflußt, ob die einzelnen Ebenen weitgehend unabhängig voneinander ihre Aufgaben erfüllen, oder ob sie über Zuteilung von Teilkompetenzen (in einem System der Politikverflechtung) in gegenseitige Abhängigkeit geraten. Ist letzteres der Fall, steigen mit der Zahl der Ebenen die Interdependenzkosten, und dies wirkt ebenfalls in Richtung auf eine Minimierung der Ebenenzahl. 35

Haben die einzelnen Ebenen des öffentlichen Sektors mehrere Aufgaben zu erfüllen, müssen allerdings Interdependenzen zwischen den Aufgaben beachtet werden. Geht man von der Situation aus, daß eine zentrale Ebene mit einer neuen, zusätzlichen Aufgabe betraut wird, so sind drei Möglichkeiten zu unterscheiden: Berührt die neue Aufgabe die bisherige Aufgabenerfüllung nicht, so sind vor allem die bisher diskutierten Kriterien der Aufgabenerfüllung zu berücksichtigen. Fördert die Erfüllung der neuen Aufgabe jedoch die der alten Aufgaben und umgekehrt, so hätte eine Zentralisierung einen zusätzlichen positiven Effekt. Behindert dagegen die neue Aufgabe die bisherige Aufgabenerfüllung, so wäre eine entsprechende Zentralisierung wesentlich kritischer zu

32 Kirsch $=$ Die Wahl $=12$.

33 "Für einen Bürger, der Mitglied von 5.000 oder gar 50.000 Kollektiven wäre, wäre es unmöglich, eine auch noch so oberflächliche, demokratische Kontrolle auszuüben." Tullock $=$ Föderalismus $=32$.

34 Vgl. dazu auch Kirsch $=$ Die Wahl $=13$.

$35 \mathrm{Vgl}$. Biehl $=$ EG-Finanzverfassung $=73$. 
betrachten. Diese Überlegungen sind für die Europäischen Gemeinschaften insofern wichtig, als dort die Aufgabe der Schaffung eines Gemeinsamen Marktes und einer freien Wettbewerbsordnung starke Auswirkungen auf viele andere Aufgabenbereiche hat.

Die Tatsache, daß eine durchgängige Aufgliederung nach dem Prinzip der fiskalischen Äquivalenz aus Praktikabilitätsgründen nicht möglich ist und in der Regel nur eine begrenzte Zahl von Ebenen gegeben ist, relativiert auch die $\mathrm{Be}-$ deutung des Prinzips der fiskalischen Äquivalenz als mögliche Begrenzung für das Subsidiaritätsprinzip. Treten Spillover-Effekte auf, die Argumente für eine Aufgabenverlagerung auf eine umfassendere Einheit liefern, ist es dennoch sinnvoll, die Aufgaben dezentral wahrzunehmen, wenn nur eine übergeordnete Einheit existiert, die die erforderliche Größe weit übertrifft.

\section{Die Notwendigkeit einer klaren Zuordnung der Aufgaben}

Eine weitere Voraussetzung für die Wirksamkeit der positiven Effekte eines föderativen Aufbaus ist eine klare Zuordnung der Aufgaben. Grundsätzlich besteht die Möglichkeit, ausschließlich einer einzigen Gebietskörperschaft eine bestimmte Kompetenz zu übertragen, oder aber eine solche Vollkompetenz in Teilkompetenzen zu unterteilen und diese verschiedenen Ebenen zuzuordnen. ${ }^{36}$

Bei der Aufgabenverteilung erscheint es zunächst immer sinnvoll, die Aufgaben säuberlich zu trennen und jeweils einer Ebene zuzuordnen. Dafür spricht vor allem der Aspekt der Einfachheit und Klarheit. ${ }^{37}$ Wird eine Aufgabe auf mehrere Ebenen verteilt, so entstehen in der Regel Koordinationsprobleme: Die Einigungs- und Entscheidungsprozesse werden komplizierter und zeitaufwendiger. Das gleichzeitige Tätigwerden verschiedener Ebenen bedingt häufig "Doppelbürokratien". ${ }^{38}$ Die Verantwortlichkeiten werden verwischt, und die fehlende Transparenz erschwert die Kontrolle durch die Parlamente und die Öffentlichkeit.

Häufig wird die Aufgabenkompetenz so aufgeteilt, daß die Gesetzgebungskompetenz und die Ausführungskompetenz unterschiedlichen Ebenen zugeordnet

36 Vgl. Biehl $=$ EG-Finanzverfassung $=74$.

37 Vgl. Fischer-Menshausen $=$ Finanzausgleich $\mathrm{II}=641$.

38 Vgl. Borell $=$ Mischfinanzierungen $=31$. 
werden. Für die Zuordnung der Ausführungskompetenz sind in der Regel andere Kriterien maßgebend als für die Entscheidungskompetenz. Bei ersterer stehen Vollzugskosten in Form von Zweckausgaben und Verwaltungsausgaben im Mittelpunkt. Diese sind häufig größenabhängig: Weist die Durchführung diseconomies of scale auf, so ist sie von dezentralen Aufgabenträgern, weist sie 0 economies of scale auf, ist sie von zentralen Aufgabenträgern zu erledigen. ${ }^{39}$ Die Gesetzgebungskompetenz und die Ausführungskompetenz werden vor allem dann getrennt, wenn erstere die Funktion einer Rahmenkompetenz erfüllen soll. "Diese Lösung liegt nahe, wenn die betreffende Gemeinschaft einerseits Wert auf nicht allzu heterogene Lösungen legt und deswegen einen einheitlichen Rahmen schafft, andererseits aber innerhalb dieses Rahmens nach wie vor Spielräume für unterschiedliche territoriale Lösungen gewährleistet sehen möchte. ${ }^{40}$

Eine Aufteilung von Entscheidungskompetenz und Ausführungskompetenz erhöht jedoch ebenfalls die Abstimmungserfordernisse zwischen den Kompetenzträgern, verwischt die Verantwortlichkeit und erschwert damit Bürgern und Parlament die Beurteilung und politische Bewertung. Man muß daher die durch eine inkongruente Verteilung von Entscheidungskompetenz und Ausführungskompetenz in der Regel erreichbare Reduzierung der Vollzugskosten gegen die bei kongruenter Verteilung in der Regel geringeren Entscheidungskosten abwägen. $\mathbf{4 1}$

\section{Das Konnexitätsprinzip}

Um die positiven Effekte eines föderativen Systems zur Geltung kommen zu lassen, müssen auch bei der Ausgabenverteilung bestimmte Bedingungen erfüllt sein. Die meisten Beiträge zur Theorie des Finanzausgleichs setzen die Aufgabenverteilung und die Ausgabenverteilung gleich. ${ }^{42}$ Dies läßt sich auf die allgemeine Erfahrung zurückführen, daß die Wirtschaftlichkeit am besten abgesichert wird, wenn die Aufgabenerfüllung mit der Kostenübernahme verbunden wird und somit die Ausgabenkompetenz der Aufgabenkompetenz folgt (Konnexitätsprinzip). "Je mehr die einzelne Gebietskörperschaft zur finanziel-

39 Vgl. Hansmeyer, Kops =Aufgabenzuständigkeit $=130 \mathrm{f}$.

40 Biehl $=$ EG-Finanzverfassung $=75$.

41 Vgl. Hansmeyer, Kops =Passiver Finanzausgleich $=11$.

42 Vgl. dazu Peffekoven $=$ Finanzausgleich $\mathrm{I}=617$. 
len Selbstverantwortung befähigt und verpflichtet wird, um so wirksamer kommen in ihrer Verwaltung und in ihren Organen der politischen Willensbildung die Kräfte zum Zuge, die den Erfordernissen einer geordneten und wirtschaftlichen Haushaltsführung Geltung zu verschaffen suchen." 43

Das Konnexitätsprinzip hängt eng mit dem Prinzip der fiskalischen Äquivalenz zusammen. In den Entscheidungsorganen einer Ebene sollten sowohl die Kostenträger als auch die Nutznießer öffentlicher Ausgaben angemessen vertreten sein. Dominieren die Zahler, so werden die einer Ebene zugeteilten Ausgabenkompetenzen in zu geringem Umfange oder mit zu niedriger Intensität genutzt. Sind dagegen die Nutznießer überproportional vertreten, werden ihre Entscheidungen tendenziell zu einem Überangebot öffentlicher Leistungen führen, da sie Kosten auf nicht beteiligte Dritte weiterwälzen können. ${ }^{44}$

Das Konnexitätsprinzip wirft jedoch dann Probleme auf, wenn die Aufgabenkompetenz nicht ungeteilt ist, also etwa die Gesetzgebungskompetenz und die Ausführungskompetenz auseinanderfallen, oder bestimmte Aufgaben von mehreren Ebenen als Gemeinschaftsaufgaben wahrgenommen werden. Im ersten Fall stellt sich die Frage, an welcher Aufgabenkompetenz - der Entscheidungs- oder der Ausführungskompetenz - die Ausgabenkompetenz anknüpfen soll. Dies wird in der Literatur unterschiedlich beantwortet. ${ }^{45}$ Die Entscheidung hängt letztlich davon $a b$, wie groß der Spielraum ist, den die beteiligten Ebenen bei der Aufgabenerfüllung haben. Wenn sämtliche Möglichkeiten der Aufgabengestaltung in der Gesetzgebungskompetenz enthalten sind und somit der Ausführungskompetenz keinerlei Gestaltungsspielräume verbleiben, so ist die Frage einfach zu beantworten: Eine Beteiligung der aufgabenvollziehenden Gebietskörperschaft ist dann nicht sinnvoll. ${ }^{46} \mathrm{Je}$ mehr Spielraum allerdings der durchführenden Ebene verbleibt, desto eher sollte sie auch die Ausgabenkompetenz erhalten.

Die Zuordnung der Ausgabenkompetenz ist auch dann schwierig, wenn sich zwei (oder sogar noch mehr) Aufgabenträger die Entscheidungskompetenz

43 Fischer-Menshausen = Finanzausgleich $\mathrm{II}=643$.

44 Vgl. Biehl, Winter $=$ EG-Finanzierung $=51 \mathrm{f}$.

45 Für die Orientierung der Ausgabenkompetenz an der Ausführungskompetenz tritt z.B. Fischer-Menshausen $=$ Finanzausgleich II $=643$ ein; Wust $=$ Föderalismus $=248$ ist dagegen der Meinung, daß die Ausgabenkompetenz der (Entscheidungs-)Gesetzgebungskompetenz folgen sollte.

46 Vgl. Hansmeyer, Kops $=$ Passiver Finanzausgleich $=13$. 


\section{Bedingungen für einen foderativen Aufbau}

teilen. Da jeder der an der Entscheidung beteiligten Aufgabenträger auf die Höhe der anfallenden Ausgaben einwirken kann, könnte eine Möglichkeit darin bestehen, die Ausgabenverteilung im Verhältnis der Entscheidungsanteile auf die Aufgabenträger vorzunehmen. 47

Mischfinanzierungen bergen jedoch stets die Gefahr, Ausgabenentscheidungen zu verzerren: Die politisch Verantwortlichen können die Vorteile der mischfinanzierten Projekte voll für sich in Anspruch nehmen, die Nachteile in Form von Kosten werden jedoch zum Teil von anderen Ebenen getragen. Diese gespaltene Kosten-Nutzen-Betrachtung der Mischfinanzierungen verleitet dazu, einer Überforderung gesamtstaatlicher Finanzierungsmöglichkeiten Vorschub zu leisten. ${ }^{48}$

\section{Die Einnahmenautonomie}

Eine weitere wichtige Forderung an einen föderativen Aufbau lautet: Jede Ebene muß in der Lage sein, ihre Einnahmen flexibel mit den Aufgaben in Einklang zu bringen. Je höher die Selbstverantwortung, desto besser die Voraussetzungen für eine kostengünstige Aufgabenerfüllung. ${ }^{49}$ Werden einer Ebene nur Erträge aus Steuern zugewiesen, über deren Erhebung auf einer anderen Ebene entschieden wird, so müssen die Ausgaben allein an den zu erwartenden, nicht zu verändernden Einnahmen ausgerichtet werden. Die Körperschaft kann dann nicht mehr frei die zugewiesenen Aufgaben und damit die Ausgaben der Höhe nach variieren. 50

Neben der Deckung der anfallenden Kosten geht es bei der Einnahmenautonomie auch darum, durch variable Einnahmenregelungen für den Bürger fühlbar zu machen, daß die Erfüllung seiner Wünsche mit einer Belastung öffentlicher Haushalte verbunden ist. Es muß eine "Verantwortungseinheit" zwischen der Belastung der Bürger (mit Abgaben) und den Ausgaben geschaffen werden. ${ }^{51}$ In einer Demokratie ist es ein eindeutiges Positivum, wenn

47 Vgl. z. B. Hansmeyer, Kops = Passiver Finanzausgleich $=14$.

48 Vgl. Füchsel = Gemeinschaftsaufgaben $=100$; zu dieser systematischen Verfälschung des Ausgabenkalküls vgl. auch Fischer $=$ Fluch des Kollektivs $=86$.

49 Vgl. Henke =Finanzierung der $\mathrm{EG}=20$.

50 Vgl. Zimmermann, Henke $=$ Finanzwissenschaft $=109$.

51 Vgl. Pfennig $=$ Finanzverfassung $=149 \mathrm{f}$. 
alle Steuerzahler sich der Tatsache bewußt sind, daß sie - in der Regel nach Maßgabe ihrer Leistungsfähigkeit - an der Finanzierung der öffentlichen Ausgaben teilnehmen müssen, die ihnen zugute kommen und über deren Erwünschtheit sie selbst entscheiden, wenn auch meist nur auf dem Wege über die von ihnen gewählten Parlamentsvertreter. Es ist für die Ausbildung und Erhaltung des Gemeinschaftsbewußtseins eine äußerst wichtige politische Aufgabe, allen Bürgern wirtschaftliche Zusammenhänge zwischen Höhe und Struktur öffentlicher Dienstleistungen einerseits und der zu ihrer Kostendeckung notwendigen Steuerforderungen andererseits immer wieder vor Augen zu führen. ${ }^{52}$ Die Merklichkeit der Steuer dient dann als "a proper check on public expenditure"53. Die ökonomische und politische Rationalität der zu bewilligenden öffentlichen Ausgaben wird von den Volksvertretern weit schärfer unter die Lupe genommen, wenn sie gewiß sind, daß sie selbst bzw. die Schichten, deren Interessen sie vornehmlich vertreten, die Zeche in Gestalt höherer Steuerverpflichtungen zu zahlen haben.

Die größtmögliche Einnahmenautonomie ist dann gegeben, wenn jede Ebene die Art der erhobenen Abgaben und deren Ausgestaltung frei bestimmen kann. ${ }^{54}$ Dies ist beim ungebundenen Trennsystem der Fall. Beim gebundenen Trennsystem dagegen werden den einzelnen Ebenen bestimmte Steuerquellen zugewiesen. Können sie dabei die Ausgestaltung der ihnen zugewiesenen Abgaben, z. B. den Steuersatz, selbst festlegen, so liegt ein gebundenes Trennsystem mit Objekthoheit vor, bei dem die Autonomie noch verhältnismäßig groß ist. Wird dagegen die Ausgestaltung zentral festgelegt (gebundenes Trennsystem ohne Objekthoheit), ist die Einnahmenautonomie schon stark eingeschränkt. Bei beiden Ausprägungen des gebundenen Trennsystems kann das Problem auftreten, daß sich die Ergiebigkeit der den Ebenen zugewiesenen Abgaben unterschiedlich entwickelt oder die Abgaben in ihrer Entwicklung nicht mit der des Finanzbedarfs übereinstimmen. Dadurch wird von Zeit zu Zeit eine Änderung der Verteilung der Abgaben erforderlich, die erfahrungsgemäß langwierige Verhandlungen und erhebliche Kosten der Konsensfindung verursacht. 55

52 Vgl. Neumark $=$ Steuerpolitik $=38 \mathrm{f}$.

53 Neumark $=$ Steuerpolitik $=39$.

$54 \mathrm{Vgl}$. Peffekoven $=$ Finanzausgleich $\mathrm{I}=619$.

55 Vgl. Peffekoven $=$ Finanzausgleich $\mathrm{I}=620$. 


\section{Bedingungen fur einen foderativen Aufbau}

Beim Zuschlagssystem kann eine Ebene prozentuale Zuschläge auf die von einer anderen Ebene erhobenen Steuern vornehmen. Kann der Zuschlag beliebig gewählt werden (ungebundener Zuschlag), ist die Einnahmenautonomie im wesentlichen gewährleistet. ${ }^{56}$ Sind die Zuschläge dagegen der Höhe nach festgelegt oder begrenzt (gebundene Zuschläge), ist die Dispositionsfreiheit stark eingeschränkt. 57

Im Rahmen des Verbundsystems wird die Steuer von einer Ebene erhoben, während die anderen Ebenen nach einer im voraus bestimmten Quote am Ertrag partizipieren. "Inwieweit die Autonomie der einzelnen Gebietskörperschaften beim Verbundsystem berücksichtigt wird, hängt davon $a b$, welches Gewicht ihnen bei der Festlegung der Quoten zukommt und inwieweit sie bei der Wahrnehmung der Objekthoheit ... beteiligt sind. "58 Wenn sich beim Verbundsystem das Verhältnis von Einnahmen zu notwendigen Ausgaben der einzelnen Ebenen unterschiedlich entwickelt, müssen Änderungen des Verteilungsschlüssels vorgenommen werden. Die Vereinbarung der Quoten birgt oft Probleme, da es schwierig ist, einen objektiven Maßstab für die Dringlichkeit staatlicher Maßnahmen zu finden. ${ }^{59}$ Außerdem wird durch die Quoten keine Differenzierung innerhalb der einzelnen Ebenen gewährleistet.

Die geringste Einnahmenautonomie ist dann gegeben, wenn eine Ebene nur Zuweisungen (eventuell sogar gebundener Art) von anderen Ebenen erhält. Sie verliert damit jegliche Möglichkeit, die Höhe ihre Einnahmen eigenständig zu variieren und damit den Aufgaben und Ausgaben anzupassen.

Bisher wurden nur die Objekthoheit und die Ertragshoheit berücksichtigt. Es ist jedoch für die Finanzautonomie durchaus auch von Bedeutung, ob eine Ebene die Verwaltungshoheit für ihre Einnahmen besitzt. Hat sie diese nicht, so ist sie zur Durchsetzung ihres Ertragsanspruchs auf die Finanzbehörden einer anderen Ebene angewiesen. Dies schwächt ihre Position, denn die andere Ebene kann Einnahmen zurückhalten oder schleppend erheben, um Druck auszuüben. Aber selbst wenn solche Fälle ausgeschlossen werden können, ist zu

56 Vgl. Nittka $=$ Finanzierungssystem $=190$.

57 Vgl. Peffekoven $=$ Finanzausgleich $\mathrm{I}=620$.

58 Peffekoven $=$ Finanzausgleich $\mathrm{I}=620$.

59 Vgl. Peffekoven $=$ Finanzausgleich $\mathrm{I}=620$. 
berücksichtigen, daß die andere Ebene nicht immer ein Interesse daran hat, eine entsprechende Steuerquelle zu pflegen und auszuschöpfen. Dies gilt vor allem beim Trennsystem, da die erhebende Ebene selbst keinen Nutzen davon hat. Insofern setzt eine starke Finanzautonomie auch voraus, daß die einzelnen Ebenen die Verwaltungshoheit über ihre Einnahmen besitzen.

Je nachdem, wie die Einnahmenzuständigkeiten den einzelnen Ebenen zugeordnet werden, ergeben sich unterschiedliche Zentralitätsprofile, in denen sich dann die Finanzverfassung in bezug auf die Einnahmenautonomie widerspiegelt. Welche Zentralitätsprofile für die Europäische Gemeinschaft und ihre Mitgliedstaaten ${ }^{\mathbf{6 0}}$ grundsätzlich möglich sind, zeigt Abbildung 7.

Beim ungebundenen Trennsystem ist - wie oben ausgeführt - die Finanzautonomie am größten, doch droht bei diesem System die Gefahr einer Mehrfachbesteuerung. Diese zieht in der Regel zusätzliche Verwaltungskosten, Steuerkonflikte, Diskriminierungen etc. nach sich. ${ }^{61}$ Eine gerechte Steuerbelastung der Bürger, die der Forderung, Bürger in der gleichen Situation sollen gleich belastet werden, entspricht, ist nicht mehr zu gewährleisten. Ein solches System führt zu einer Vielzahl von Einzelsteuern, "so daß von einem rationalen Steuersystem meist nicht die Rede sein kann"62. Dem - durchaus berechtigten - Wunsch nach fiskalischer Eigenverantwortung werden insofern Grenzen gesetzt.

60 Die föderative Struktur der Mitgliedstaaten wird dabei außer acht gelassen.

61 Vgl. Kamp, Langheinrich, Stamm =Optimales Finanzsystem $=59$.

62 Peffekoven $=$ Finanzausgleich $\mathrm{I}=619$. 
Abbildung 7: Die Zuordnung von Einnahmenzuständigkeiten auf die Europäische Gemeinschaft (EG) und die Mitgliedstaaten (MS)

\begin{tabular}{|c|c|c|c|c|c|c|c|c|c|c|c|c|}
\hline \multirow{3}{*}{$\begin{array}{l}\text { System der } \\
\text { Einnahmen- } \\
\text { verteilung }\end{array}$} & \multirow{3}{*}{\multicolumn{2}{|c|}{$\begin{array}{l}\text { Zuweisungs- } \\
\text { system von } \\
\text { unten nach } \\
\text { oben }\end{array}$}} & \multirow{3}{*}{\multicolumn{2}{|c|}{$\begin{array}{l}\text { Verbund- } \\
\text { system }\end{array}$}} & \multirow{3}{*}{\multicolumn{2}{|c|}{$\begin{array}{l}\text { Zuschlags- } \\
\text { system }\end{array}$}} & \multicolumn{6}{|c|}{ Trennsystem } \\
\hline & & & & & & & \multicolumn{4}{|c|}{ gebunden } & \multicolumn{2}{|c|}{$\begin{array}{l}\text { unge- } \\
\text { bunden }\end{array}$} \\
\hline & & & & & & & ohn & $\mathrm{eOH}$ & & $\mathrm{OH}$ & & \\
\hline $\begin{array}{l}\text { Einnahmen- } \\
\text { zuständigkeit }\end{array}$ & EG & MS & EG & MS & EG & MS & EG & MS & EG & MS & EG & MS \\
\hline $\begin{array}{l}\text { Objekthoheit } \\
\text {-über die Bemes- } \\
\text { sungsgrundlage } \\
\text {-über den Steuer- } \\
\text { satz }\end{array}$ & & $\mathbf{x}$ & & $x$ & + & $\mathbf{x}$ & & $\mathbf{x}$ & & $\mathbf{x}$ & + & + \\
\hline Ertragshoheit & & $\mathbf{x}$ & & $\mathbf{x}$ & + & $\mathbf{x}$ & + & & & & & \\
\hline $\begin{array}{l}\text { Durchführungs- } \\
\text { hoheit }\end{array}$ & & $\mathbf{x}$ & & $\mathbf{x}$ & & $\mathbf{x}$ & $(+)$ & & $(+)$ & & $(+)$ & $\mathbf{x}$ \\
\hline
\end{tabular}

Quelle: Henke $=$ Finanzierung der EG $=17$. Die Originalabbildung wird hier in überarbeiteter und ergänzter Fassung wiedergegeben. 


\section{Ansätze zu einer "gerechten" Verteilung der Finanzierungslasten}

Das Zuweisungssystem, das nur eine geringe Einnahmenautonomie zuläßt, ist für die Finanzierung internationaler Organisationen sehr verbreitet. ${ }^{63}$ Durch die Gewährung von Finanzierungsbeiträgen behält der einzelne Mitgliedstaat relativ große Einflußmöglichkeiten. Bei der Festlegung der Zuweisungen muß nicht nur bestimmt werden, in welchem Umfang Mittel an die internationale Organisation fließen, sondern es muß auch ausgehandelt werden, wie groß der Finanzierungsanteil des einzelnen Mitgliedstaates sein soll. Es müssen Kriterien für die angemessenen Leistungen der Mitgliedstaaten gefunden werden. Dabei lassen sich zwei Konzeptionen unterscheiden. ${ }^{64}$ Der ersten liegt die Vorstellung zugrunde, daß die Mitgliedstaaten solcher Organisationen entweder entsprechend den anteiligen Nutzen, die ihnen aus der Mitgliedschaft erwachsen (Nutzenäquivalenz), oder gemäß den anteiligen Kosten für beanspruchte Leistungen (Kostenäquivalenz) an der Finanzierung beteiligt werden. ${ }^{65}$ Die Problematik dieses Ansatzes liegt in der Ermittlung und dem Vergleich der anteiligen Nutzen oder Kosten. Bei den Beitragsfestsetzungen könnten z. B. einige Mitgliedstaaten unter der Zielsetzung, den eigenen Finanzierungsanteil zu senken, die Strategie verfolgen, die anderen Verhandlungspartner nicht vollständig oder nicht wahrheitsgemäß über die eigenen Präferenzen zu informieren. Um die Vorteile und Nachteile der Integration zu ermitteln und vergleichbar zu machen, müßten objektivierbare Maßstäbe zur Verfügung stehen. Dies ist in der Regel nicht der Fall. ${ }^{66}$ Insbesondere dann, wenn eine Organisation ein breites Aufgabenspektrum durchführt, wird man kaum einen geeigneten Indikator für die Abschätzung der Nutzen oder Kosten finden können. 67

63 Vgl. dazu auch Peffekoven $=$ Eigene Einnahmen $=316 \mathrm{ff}$.

64 Vgl. dazu Nittka $=$ Finanzierungssystem $=225 \mathrm{ff}$., Biehl $=$ Finanzausgleich IV $=700$, Henke $=$ Finanzierung der $E G=34 \mathrm{ff}$. und Hackenbroch $=$ Supranationaler Finanzausgleich $=112 \mathrm{ff}$.

65 Vgl. Nittka $=$ Finanzierungssystem $=231$.

66 Vgl. Nittka $=$ Finanzierungssystem $=231$.

67 Vgl. Peffekoven $=$ Eigene Einnahmen $=317$. 
Die zweite Konzeption geht davon aus, daß die Mitgliedstaaten gemäß ihrer Wirtschaftskraft ${ }^{68}$ zur Finanzierung herangezogen werden. Dies erfordert zunächst, daß die Frage nach dem Indikator für die Wirtschaftskraft gelöst werden muß. Dabei kann auf unterschiedliche Maßstäbe zurückgegriffen werden, etwa auf Sozialproduktsgrößen (Brutto- oder Nettosozialprodukt zu Marktpreisen, Volkseinkommen), auf den privaten Konsum oder auf andere Größen wie das Volksvermögen, die Bevölkerung, die Devisenreserven, den Staatsanteil, die Wettbewerbsintensität, die Steuerkraft oder das Produktionspotential. Auch ein mehrdimensionaler Indikator ist vorstellbar. ${ }^{69}$ In der Praxis werden allerdings in den meisten Fällen Sozialproduktsgrößen herangezogen. ${ }^{70}$ Probleme können sich dabei durch unterschiedliche nationale Definitions-, Erfassungs- und Bewertungsfragen ergeben sowie bei der Bestimmung des "richtigen" Umrechnungskurses, also der Frage, wie die in nationalen Werteinheiten ausgedrückten Sozialproduktsgrößen in eine einheitliche Werteinheit umzurechnen sind. ${ }^{71}$ Bei Mitgliedstaaten unterschiedlicher Größe kann die Leistungskraft der Volkswirtschaften nur angemessen beurteilt werden, wenn Pro-Kopf-Größen herangezogen werden. Wie die Lastverteilung innerhalb der einzelnen Mitgliedstaaten geregelt wird, interessiert beim Vergleich der Wirtschaftskraft nicht. Die Tatsache, daß es im gesamten föderativen System dazu kommen kann, daß der "arme" Bürger des "reichen" Mitgliedstaates belastet wird, um "reiche" Bürger des "armen" Mitgliedstaates zu alimentieren, ist lediglich unter dem Blickwinkel der individuellen Leistungsfähigkeit problematisch, nicht jedoch für den Finanzausgleich zwischen den Mitgliedstaaten. ${ }^{72}$

Die konkrete Beitragszumessung an die einzelnen Mitgliedstaaten erfolgt in der Regel proportional zur Wirtschaftskraft. Eine progressive Lastverteilung kann

68 Eine Reihe von Autoren sprechen hier vom "Leistungsfähigkeitsprinzip", u. a. Nittka $=$ Finanzierungssystem $=233 \mathrm{ff}$. und Hackenbroch $=$ Supranationaler Finanzausgleich $=117 \mathrm{ff}$. Da jedoch zwischen dem Leistungsfähigkeitsprinzip als Grundsatz für die Steuerlastverteilung auf Individuen und der Verteilung der Finanzbeiträge zwischen den Mitgliedstaaten entsprechend der wirtschaftlichen Lage wichtige Unterschiede bestehen, wird dieser Terminologie nicht gefolgt.

$69 \mathrm{Vgl}$. Henke =Finanzierung der $\mathrm{EG}=35$.

70 Vgl. Hackenbroch $=$ Supranationaler Finanzausgleich $=118 \mathrm{f}$.

71 Vgl. Nittka $=$ Finanzierungssystem $=235$.

72 Zum höheren Aussagewert von wirtschaftlichen Quoten gegenüber absoluten Zahlen vgl. auch Wissenschaftlicher Beirat beim Bundesministerium der Finanzen =Aussagefähigkeit staatswissenschaftlicher Quoten $=849 \mathrm{ff}$. 
auf internationaler Ebene noch viel weniger eindeutig begründet werden als bei der Steuerlastverteilung innerhalb eines Mitgliedstaates auf dessen Bürger. Der auf nationaler Ebene letztlich politisch ausgehandelte Progressionsgrad hängt vor allem von der Bereitschaft zur Solidarität ab. Da diese zwischen Staaten in der Regel geringer ist als innerhalb der einzelnen Staaten, erstaunt es nicht, daß bei internationalen Organisationen in der Regel nur ein proportionaler Tarif bei der Lastenverteilung angewandt wird.

\section{Zur Notwendigkeit eines Finanzausgleichs im engeren Sinne}

Eine noch so gute Regelung der Aufgaben-, Ausgaben- und Einnahmenverteilung (Finanzausgleich im weiteren Sinne) kann nicht immer sicherstellen, daß jede Gebietskörperschaft einer Ebene in der Lage ist, ihre Aufgaben bei gleicher Steueranspannung aus den ihr zugewiesenen Einnahmenquellen zu finanzieren. Unterschiede in bezug auf periphere oder zentrale Lage, Bevölkerungsdichte und Siedlungsstruktur, sektorale Wirtschaftsstruktur und Infrastrukturausstattung können zu erheblichen Abweichungen in der Steuerkraft einzelner Gebiete führen. ${ }^{73} \mathrm{Um}$ sicherzustellen, daß auch finanzschwache Gebietskörperschaften in der Lage sind, ihren Aufgaben nachzukommen, werden sie in der Regel mit finanziellen Zuwendungen unterstützt. Dahinter steht die Überlegung, daß ein föderatives Gemeinwesen langfristig nur stabil sein kann, wenn es sich auf die Zustimmung der Mehrheit der Bürger in allen Teilregionen stützen kann. Eine solche Zustimmung ist nur dann zu erwarten, wenn bei den einzelnen Körperschaften die Versorgung mit öffentlichen Leistungen sowie die Belastung mit Steuern nicht zu sehr auseinanderklaffen. ${ }^{74}$

Geht es nicht nur um die Stabilität einer bestehenden, sondern um die fortschreitende Integration einer sich entwickelnden Gemeinschaft, so sind Zuweisungen noch unter einem anderen Aspekt zu sehen: Selbst wenn die Integration insgesamt Vorteile bringt, können diese unterschiedlich auf die einzelnen Gebietskörperschaften verteilt sein, ja es mag sein, daß einige Gebietskörperschaften zunächst Nachteile in Kauf nehmen müssen. Um diese benachteiligten Gebietskörperschaften dennoch für weitere Integrationsschritte zu gewinnen, werden ihnen Zuweisungen gewährt. ${ }^{75}$

73 Vgl. Biehl, Winter =EG-Finanzierung $=53 \mathrm{f}$.

74 Vgl. Peffekoven $=$ Finanzausgleich $\mathrm{I}=628$.

75 Vgl. Reding $=$ Problematik eines Finanzausgleichs $=203$. 


\section{Bedingungen für einen foderativen Aufbau}

Die Höhe der Zuweisungen hängt einerseits vom Ausmaß der Solidarität $a b$, andererseits aber auch davon, inwieweit die Empfänger von Zuweisungen verpflichtet werden können, diese in einem bestimmten Sinne zu verwenden. Man kann davon ausgehen, daß Gebietskörperschaften desto eher zu Finanzzuweisungen bereit sind, je mehr sie damit rechnen können, daß ihre Mittel im Rahmen eines gemeinsamen Verfassungs- und Rechtssystems nur für allgemein akzeptierte Zwecke verwendet werden. Ist dieser gemeinsame Verfassungsrahmen nicht oder unvollständig vorhanden, haben ungebundene Finanzzuweisungen hohe Präferenzkosten zur Folge und ein explizites System eines Finanzausgleichs i. e. S. ist nicht zu erwarten. In diesem Fall ist auch die Sensibilität der betroffenen Gebietskörperschaften für die regionalen Verteilungseffekte der Einnahmen und Ausgaben sehr hoch. Während innerhalb eines Staates mit vollentwickeltem Verfassungssystem sogar auf die Kenntnis der Verteilungseffekte von Ausgaben weitgehend verzichtet werden kann, wenn man sicher ist, daß die Mittel dazu "gerecht" aufgebracht wurden, werden beim Fehlen eines solchen Konsensrahmens auch die Ausgabeneffekte mißtrauischer verfolgt. ${ }^{76}$

Der Nettoeffekt der Verteilungswirkungen von Ausgaben und Einnahmen, die zumindest primär keine Verteilungsziele verfolgen, kann in Anlehnung an Biehl und Winter als impliziter Finanzausgleich $i$. e. S. bezeichnet werden. ${ }^{77}$ Besteht kein expliziter Finanzausgleich i. e. S., so treten diese Verteilungswirkungen in der politischen Diskussion besonders in den Vordergrund. Die Diskussion um die Verteilungseffekte der Ausgaben und Einnahmen der Europäischen Gemeinschaften (Nettopositionen) muß insbesondere unter diesem Aspekt betrachtet werden.

$76 \mathrm{Vgl}$. Biehl $=$ EG-Finanzverfassung $=77$.

77 Vgl. Biehl, Winter $=$ EG-Finanzierung $=54$. 


\section{DIE REGELUNGEN DES FINANZAUSGLEICHS IN DEN EURO- PÄISCHEN GEMEINSCHAFTEN UND IHRE AUSWIRKUNGEN AUF DIE BUNDESREPUBLIK DEUTSCHLAND}

Nach den grundsätzlichen Überlegungen zur Gestaltung eines föderativen Systems sollen nun die wichtigsten Regelungen des Finanzausgleichs in den Europäischen Gemeinschaften erläutert werden. Diese Darstellung stellt die Grundlage für Teil D dieser Arbeit dar, in dem die Probleme des Finanzausgleichs in den Europäischen Gemeinschaften unter besonderer Berücksichtigung des föderativen Aufbaus in der Bundesrepublik Deutschland herausgearbeitet werden sollen. Obwohl dort nicht alle Regelungen wieder aufgegriffen werden, sondern nur jene, an denen sich die Probleme besonders gut verdeutlichen lassen und die ein entsprechend großes Gewicht aufweisen, ist es hier sinnvoll, einen umfassenderen Überblick zu geben; denn erstens sind auch diese Einzelregelungen in den Gesamtzusammenhang eingebettet und zweitens kann nur so die Breite der Aufgabenverlagerung von den nationalen Ebenen auf die Europäischen Gemeinschaften deutlich gemacht werden.

\section{Rechtliche und institutionelle Grundlagen der Europäischen Gemein- schaften}

\section{Die Verträge}

Die Ursprünge der Europäischen Gemeinschaften gehen auf den 1951 unterzeichneten Vertrag über die Gründung der Europäischen Gemeinschaft für Kohle und Stahl zurück. Die fortschreitende Integration führte 1957 zur Unterzeichnung der Verträge zur Gründung der Europäischen Wirtschaftsgemeinschaft und der Europaischen Atomgemeinschaft. Diese Verträge bilden im wesentlichen die Grundlage für die Politik der Gemeinschaft. Seit dem Vertrag zur Einsetzung eines gemeinsamen Rates und einer gemeinsamen Kommission der Europäischen Gemeinschaften, der am 1.7.1967 in Kraft getreten ist, sind die wichtigsten Organe der drei rechtlich selbständigen Gemeinschaften zusammengelegt; nicht zuletzt deshalb spricht man inzwischen auch von der "Europäischen Gemeinschaft". Die Verträge haben seit der Gründung der Gemeinschaften immer wieder Änderungen und Erweiterungen erfahren. Die bedeutendste Weiterentwicklung stellte die Einheitliche Europäische Akte dar, die am 1.7.1987 in Kraft trat und die Befugnisse der Gemeinschaft deutlich erweiterte. 


\section{Die Zusammensetzung der Organe der Europäischen Gemeinschaften}

Die Organe der Gemeinschaften sind die Kommission, der Rat, das Europäische Parlament und der Europäische Gerichtshof. Da die Kommission, der Rat und das Parlament im folgenden von besonderer Bedeutung sind, soll hier kurz ihre Zusammensetzung erläutert werden. Welche Zuständigkeiten diese Organe haben, wird bei der Betrachtung der Aufgabenverteilung, der Ausgabenverteilung und schließlich auch bei der Einnahmenverteilung näher erläutert werden.

Die Kommission besteht aus 17 Mitgliedern, die aufgrund ihrer allgemeinen Befähigung ausgewählt werden und volle Gewähr für ihre Unabhängigkeit bieten müssen. ${ }^{1}$ Die Mitglieder der Kommission werden von den Regierungen der Mitgliedstaaten in gegenseitigem Einvernehmen ernannt. Ihre Amtszeit beträgt vier Jahre. Eine Wiederernennung ist zulässig. ${ }^{2}$ Die fünf großen Mitgliedstaaten (Deutschland, Frankreich, Großbritannien, Italien und Spanien) stellen je zwei, die sieben kleinen Mitgliedstaaten je einen Kommissar.

Der Rat besteht aus Vertretern der Mitgliedstaaten. Jede Regierung entsendet eines ihrer Mitglieder. ${ }^{3}$ Grundsätzlich steht es jedem Mitgliedstaat frei zu entscheiden, welches Regierungsmitglied er entsenden will; in der Regel wird der für den jeweiligen Beratungsgegenstand zuständige nationale Fachminister mit der Vertretung beauftragt. Man spricht dann vom "Ministerrat". 4 Mindestens zweimal im Jahr tritt darüber hinaus der Europäische Rat zusammen, bei dem sich die Staats- und Regierungschefs der Mitgliedstaaten sowie der Präsident der Kommission treffen. Sie werden von den Ministern für auswärtige Angelegenheiten und einem Mitglied der Kommission unterstützt. ${ }^{5}$ Der Europäische

1 Art. 10 Abs. 1 Satz 1 des Vertrages zur Einsetzung eines gemeinsamen Rates und einer gemeinsamen Kommission der Europäischen Gemeinschaften.

2 Art. 11 des Vertrages zur Einsetzung eines gemeinsamen Rates und einer gemeinsamen Kommisson der Europäischen Gemeinschaften.

3 Vgl. Art. 2 Satz 1 des Vertrages zur Einsetzung eines gemeinsamen Rates und einer gemeinsamen Kommission der Europäischen Gemeinschaften.

4 "Staatssekretäre, auch die nichtparlamentarischen der Bundesrepublik Deutschland, werden nach einer schon im Rahmen der EGKS ständig geübten Praxis als Mitglieder ihrer Regierung angesehen, obwohl sie nach Art. 62 GG nicht Mitglieder der Bundesregierung sind." Ehlermann, Bieber =Handbuch des Europäischen Rechts $=$ Teil I A $61,9 f$.

5 Vgl. Art 2, Satz 1 und 2 der Einheitlichen Europäischen Akte. 
Rat berät über Fragen der Weiterentwicklung der Europäischen Gemeinschaften und faßt gegebenenfalls politische Grundsatzbeschlüsse.

Die Abgeordneten des Europäischen Parlaments werden von den wahlberechtigten Bürgern in den Mitgliedstaaten in allgemeiner, unmittelbarer Wahl gewählt. ${ }^{6}$ Die Zahl der in jedem Mitgliedstaat gewählten Abgeordneten ist nach der Größe der Bevölkerung differenziert, allerdings entsenden die bevölkerungsreichen Mitgliedstaaten relativ weniger Abgeordnete als die bevölkerungsarmen. ${ }^{7}$ Die Abgeordneten werden auf fünf Jahre gewählt; ${ }^{8}$ sie sind weder an Aufträge noch an Weisungen gebunden. ${ }^{9}$

\section{Der Rechtsetzungsprozeß der Europäischen Gemeinschaften}

\section{a) Das Zusammenwirken der Organe beim Rechtsetzungsprozeß}

In der Regel gehen Rechtsakte der Gemeinschaft auf Vorschläge der Kommission zurück. Durch das Vorschlagsrecht sollte die Kommission eigentlich "Initiator der Gemeinschaftspolitik" werden. ${ }^{10}$ Das Initiativrecht der Kommission wurde jedoch zunächst 1966 durch den sogenannten Luxemburger Kompromiß stark abgeschwächt, in dem eine Konsultationspflicht der Kommission gegenüber den Regierungen der Mitgliedstaaten vor der Annahme von Vorschlägen durch die Kommission festgelegt wurde. Die Kommission vermag ihren Vorschlägen nicht immer solche politische Autorität zu verleihen, daß sie im Rat Konsens finden. "Stattdessen bestimmt häufig das vermutete Maß des im Rat Durchsetzbaren den Inhalt eines Vorschlags. "11 Im Zusammenhang mit der Errichtung des Binnenmarktes und den Plänen zur Weiterentwicklung der Gemeinschaft zur Wirtschafts- und Währungsunion hat die Bedeutung der Kommission allerdings deutlich zugenommen. Das politische Gewicht der

6 Art. 1 des Beschlusses über die Einführung allgemeiner unmittelbarer Wahlen der Abgeordneten der Versammlung.

7 Die genauen Zahlen sind in Art. 2 des Beschlusses über die Einführung allgemeiner unmittelbarer Wahlen der Abgeordneten der Versammlung aufgeführt.

8 Art. 3 Abs. 1 des Beschlusses über die Einführung allgemeiner unmittelbarer Wahlen der Abgeordneten der Versammlung.

9 Art. 4 Abs. 1 Satz 2 des Beschlusses über die Einführung allgemeiner unmittelbarer Wahlen der Abgeordneten der Versammlung.

$10 \mathrm{Vgl}$. Harbrecht $=\mathrm{EG}=85$.

11 Beutler u. a. =Europäische Gemeinschaft $=131$. 
Kommission scheint auch vom Ansehen und dem persönlichen Durchsetzungsvermögen ihrer Mitglieder abzuhängen.

Formal ist die Kommission unabhängig; den Mitgliedstaaten ist jeder Versuch der Einflußnahme verboten. "Da aber die Kommission nicht aus Wahlen hervorgegangen und auch über keine sonstige eigenständige Legitimation bei den gesellschaftlichen Kräften in den Mitgliedstaaten verfügt, bleibt sie darauf angewiesen, Legitimation für ihre Entscheidungen aus den sie ernennenden Gremien, also den nationalen Regierungen, abzuleiten. Der Sachverstand ihres bürokratischen Apparats kann diese Legitimation nicht ersetzen. Es bedarf daher gar nicht des Versuchs der Regierungen, ein bestimmtes Kommissionsmitglied zu beeinflussen. Schon durch Auswahl, Ernennung (und das Bemühen um die Unterstützung der Regierungen für die spätere berufliche Tätigkeit) sind die Kommissare mit den Ministerien und Regierungen ihres Herkunftslandes verbunden. Dies fördert die Beibehaltung informeller Kontakt- und damit Einflußmöglichkeiten. "12

Wird der Einfluß der Regierungen der Mitgliedstaaten schon bei der formal unabhängigen Kommission deutlich, so ist der Rat von vornherein als Instrument für die Mitwirkung der nationalen Regierungen am gemeinschaftlichen Entscheidungsprozeß konzipiert. Der Rat besitzt die allgemeine Beschlußkompetenz über die von der Kommission vorgeschlagenen Rechtsakte. Dadurch bestimmt er letztlich die gemeinschaftliche Politik. ${ }^{13}$ Bei der Beschlußfassung ist der Rat normalerweise nicht an bestimmte Fristen gebunden, was manche der langen Zeitspannen zwischen Vorlage und Verabschiedung von Rechtsakten erklärt. ${ }^{\mathbf{1 4}}$ Der Rat sollte prinzipiell mit der Mehrheit seiner Mitglieder beschließen; ${ }^{15}$ bei vielen Entscheidungen, vor allem bei Fragen der Rechtsangleichung nach Art. 100 EWGV, wird den Mitgliedstaaten jedoch ein Vetorecht eingeräumt, so daß hier die Entscheidungen einstimmig getroffen werden müssen. Der Rat tagt in nicht-öffentlichen Sitzungen.

Das Europäische Parlament hat im Vergleich zum Rat nur bescheidene Kompetenzen. So darf das Europäische Parlament zwei klassische parlamentarische Aufgaben überhaupt nicht wahrnehmen, nämlich Gesetze initiieren und die

12 Beutler u. a. =Europäische Gemeinschaft $=130$.

$13 \mathrm{Vgl}$. Harbrecht $=\mathrm{EG}=88$.

14 Vgl. dazu auch Ebers =Institutionelle Struktur $=361$.

15 Vgl. Art. 148 Abs. 1 EWGV. 
Exekutive wählen. 16 Vor der Verabschiedung der Einheitlichen Europäischen Akte besaß es nur das Recht der Anhörung. Durch die Einheitliche Europäische Akte wurde ein neues Kooperationsverfahren zwischen Rat und Parlament im Vertrag zur Gründung der Europäischen Wirtschaftsgemeinschaft verankert. Dabei legt der Rat nach Stellungnahme des Parlaments mehrheitlich einen gemeinsamen Standpunkt fest, den er dem Parlament zuleitet. Der Rat muß dabei das Parlament in allen Einzelheiten über die Gründe für seinen Standpunkt unterrichten. Wenn das Parlament den Standpunkt des Rates nicht billigt, kann es innerhalb von drei Monaten mit absoluter Mehrheit Abänderungen am gemeinsamen Standpunkt des Rates vorschlagen oder diesen völlig ablehnen. Ist letzteres der Fall, so kann der Rat in zweiter Lesung nur einstimmig beschließen. Wird der Standpunkt nicht völlig abgelehnt, schlägt das Parlament also lediglich Änderungen vor, so werden diese Vorschläge zuerst von der Kommission geprüft. Die Kommission übermittelt dann dem Rat zusammen mit dem von ihr geprüften Vorschlag die von ihr nicht übernommenen Abänderungen des Parlaments und nimmt dazu Stellung. Der Rat verabschiedet mit qualifizierter Mehrheit den von der Kommission überprüften Vorschlag, ändern kann er ihn nur einstimmig (Art. 149 EWGV). ${ }^{17}$

Trotz der verstärkten Kooperation mit dem Parlament bleibt der Ministerrat das letztlich entscheidende Gremium im Rahmen des Rechtsetzungsprozesses in den Gemeinschaften.

Hier liegt ein fundamentaler Unterschied zu den bundesstaatlichen Konstruktionen in föderativen Staaten, etwa der Bundesrepublik Deutschland. Formal läßt sich zwar eine bestimmte Ähnlichkeit feststellen: hier Bundestag, dort Europäisches Parlament; hier Bundesrat (Vertreter der Regierungen der Länder), dort Rat. Doch in der Bundesrepublik Deutschland ist der Bundestag das wichtigste Legislativorgan. In den Europäischen Gemeinschaften - die eben nur einen Staatenbund darstellen - nimmt demgegenüber der Rat im wesentlichen die legislativen Aufgaben wahr, obgleich er aus Vertretern der nationa-

$16 \quad$ Vgl. Ebers $=$ Institutionelle Struktur $=362$.

17 Seit Inkrafttreten der Einheitlichen Europäischen Akte wurden 112 Vorschläge des Parlaments angenommen. "Von der Gesamtzahl der in erster Lesung vom Parlament eingebrachten Anderungsanträge wurden mehr als $66 \%$ von der Kommission und etwa $48 \%$ vom Rat übernommen. In zweiter Lesung hat das Parlament 44 gemeinsame Standpunkte des Rates gebilligt und zu 68 Standpunkten Abänderungen vorgeschlagen; von diesen Abänderungen wurden mehr als $49 \%$ von der Kommission und fast $14 \%$ vom Rat übernommen. " Kommission der EG $=$ XXIII. Gesamtbericht $=31$. 
len Exekutivorgane besteht. Das bedeutet: Die Gemeinschaften kennen zur Zeit keine unmittelbar demokratisch legitimierte gesetzgebende Körperschaft. Damit entsprechen die institutionellen Verhältnisse in den Europäischen Gemeinschaften nicht den prinzipiellen Vorstellungen von Gewaltenteilung.

Für die Weiterentwicklung der Gemeinschaften zu einer politischen Union werden zwar erweiterte Befugnisse für das Europäische Parlament diskutiert, die bisherigen Vorschläge laufen jedoch eher auf mehr Macht für den Rat, vor allem für den Europäischen Rat der Staats- und Regierungschefs, hinaus. Im Zusammenhang mit dem Europäischen Parlament sind lediglich ein erweitertes Recht auf Mitentscheidung und ein Recht auf Mitwirkung bei der Bestellung des Kommissionspräsidenten im Gespräch. ${ }^{18}$ Insgesamt bleibt damit auf absehbare Zeit ein großer Spielraum für die Einflußnahme der nationalen Regierungen auf die Entscheidungen der Europäischen Gemeinschaften bestehen.

\section{b) Die Instrumente der Rechtsetzung}

Nach Art. 189 EWGV erläßt die Gemeinschaft zur Erfüllung ihrer Aufgaben Verordnungen, Richtlinien und Entscheidungen. Verordnungen haben allgemeine Geltung, sind in allen ihren Teilen verbindlich und gelten unmittelbar in jedem Mitgliedstaat. Die Entscheidung regelt einen Einzelfall; dabei ist sie ebenfalls in allen ihren Teilen für denjenigen verbindlich, an den sie gerichtet ist.

Das bevorzugte Instrument der Europäischen Gemeinschaften ist jedoch die Richtlinie. ${ }^{19}$ Richtlinien sind für jeden Mitgliedstaat hinsichtlich des zu erreichenden Ziels verbindlich, überlassen jedoch den innerstaatlichen Stellen die Wahl von Form und Mitteln zur Erreichung der Ziele. ${ }^{20}$

Damit wird ein zweistufiges Rechtsetzungsverfahren geschaffen, bei dem die Gemeinschaften lediglich einen bestimmten Rahmen vorgeben. Die Regelungen einer Richtlinie treten nicht an die Stelle der nationalen Rechtsvorschriften, sondern die Mitgliedstaaten werden verpflichtet, ihr Recht an die Bestimmungen der Richtlinie anzupassen. ${ }^{21}$

$18 \mathrm{Vgl}$. Hort =Europa in neuer Gestalt $=1$ und o. V. $=$ Zuständigkeiten $=2$.

19 Vgl. Schmidhuber $=$ Gesetzgebung $=75$.

20 Vgl. Art. 189 Satz 3 EWGV.

21 Vgl. Beutler u. a. =Europäische Gemeinschaft $=76$. 
Diese Zweistufigkeit führt dazu, daß sich die Zahl der Rechtsetzungsakte insgesamt um den "nationalen Faktor" erhöht. 22 Die Mitgliedstaaten sind damit nicht nur über den Ministerrat als dem Entscheidungsorgan am Erlaß der Richtlinien beteiligt, sondern darüber hinaus in eigener Verantwortung zuständig für die Umsetzung der Richtlinien in nationales Recht. Während die Teilnahme an der Rechtsetzung der Europäischen Gemeinschaften durch die Exekutive - also in der Bundesrepublik Deutschland durch die Bundesregierung - erfolgt ${ }^{23}$, ist für die Umsetzung der Richtlinien in nationales Recht die Legislative - in der Bundesrepublik Deutschland in den meisten Fällen der Bundestag $^{24}$, bei reinen Länderangelegenheiten in seltenen Fällen auch die Landesparlamente - zuständig.

Die Rechtsetzung der Gemeinschaften ist normalerweise ein Kompromiß zwischen den Interessen der zwölf Mitgliedstaaten. Die Umsetzung der Richtlinien wird dagegen im wesentlichen dadurch bestimmt, welche Spielräume für die Einbringung eigener nationaler Vorstellungen die Richtlinie den Mitgliedstaaten noch beläßt. ${ }^{25}$ Nach dem seit 1985 geltenden "neuen Ansatz" ${ }^{26}$ soll die Gesetzgebung der Gemeinschaften darauf gerichtet sein, nur die zentralen Fragen eines Rechtsbereichs auf Gemeinschaftsebene zu harmonisieren. Im übrigen soll vom Prinzip der gegenseitigen Anerkennung gleichwertiger nationaler Regelungen ausgegangen werden, so daß dem nationalen Gesetzgeber ein Spielraum bleibt. In letzter Zeit hat sich jedoch gezeigt, daß diese Absicht nicht realisiert wurde und Richtlinien stark "zur Fixierung von Einzelheiten tendieren, die einer Ausgestaltung durch die Mitgliedstaaten kaum mehr Raum lassen, und sie also wie Verordnungen praktisch unverändert übernommen werden"27.

Wenn die Richtlinien Gestaltungsspielräume bei der Umsetzung offenlassen, ermöglichen sie zwar eine gewisse Flexibilität bei der Anpassung gemeinschaftlicher Regelungen an die besonderen Verhältnisse in den Mitgliedstaa-

22 Vgl. Schmidhuber $=$ Gesetzgebung $=76$.

23 Vitzthum spricht hier von "Exekutivföderalismus". Vitzthum =Föderalismus $=286$.

24 Vgl. Schmidhuber $=$ Gesetzgebung $=76$.

25 Vgl. Schmidhuber $=$ Gesetzgebung $=76$.

26 Rat der EG = Technische Harmonisierung und Normung $1985=1 \mathrm{ff}$.

27 Pag =Umsetzung von EG-Vorschriften= 128; Schweitzer bezeichnet derart konkretisierte Richtlinien als "self-executing", Schweitzer =Bundesstaat und EG = 17 . 
ten, ${ }^{28}$ ihre Effizienz wird allerdings in Zweifel gezogen, weil die Umsetzung in nationales Recht oft langwierig ist. ${ }^{29}$ Im Bericht über die Kontrolle der Anwendung des Gemeinschaftsrechtes ${ }^{\mathbf{3 0}}$ stellte die Kommission fest, daß bei knapp 290 von über 780 Richtlinien die Mitgliedstaaten noch nicht die Maßnahmen zu ihrer nationalen Anwendung ergriffen haben, obwohl die Übergangsfristen bereits abgelaufen sind. ${ }^{31}$ Vor allem im Zusammenhang mit der Vollendung des Binnenmarktes wird die mangelnde Umsetzung in nationales Recht beklagt.

\section{Die Durchführung von Rechtsakten der Europäischen Gemeinschaften}

Der Grundsatz der enumerativen Einzelermächtigung gilt prinzipiell auch für die funktionelle Zuständigkeit der Gemeinschaften. Soweit sie sachlich eine Kompetenz besitzt, ist sie in der Regel lediglich für die Rechtsetzung und nur, wenn dies ausdrücklich vorgesehen ist, auch für die Durchführung zuständig. ${ }^{32}$ Die wichtigsten Bereiche, in denen dieser Fall auftritt, sind die Verwaltung der Gemeinschaftsfonds, die Beihilfenaufsicht und die Forschungsförderung.

Die nationalen Ebenen handeln bei der Durchführung von Rechtsakten der Gemeinschaften weitgehend aufgrund eigener mitgliedstaatlicher Zuständigkeit. "In den Mitgliedstaaten ändert die Durchführung von Gemeinschaftsrecht nicht die bestehende interne Zuständigkeitsverteilung. "33 Für die Durchführung von Gemeinschaftsrecht ist somit nicht automatisch der Zentralstaat - in der Bundesrepublik Deutschland der Bund - zuständig; die Durchführungskompetenz der deutschen Länder bleibt prinzipiell bestehen.

28 Vgl. Pag $=$ Umsetzung von EG-Vorschriften $=128$.

29 Vgl. Beutler u. a. =Europäische Gemeinschaft $=185$.

30 Vgl. Kommission der EG = Anwendung des Gemeinschaftsrechtes 1986=1ff.

31 Dabei ist zu beachten, daß die Umsetzung in den einzelnen Mitgliedstaaten sehr unterschiedlich gehandhabt wird, vgl. Philipp =Umsetzung von EG-Richtlinien $=12$.

32 Vgl. Beutler u. a. =Europäische Gemeinschaft $=76$.

33 Beutler $u$. a. $=$ Europäische Gemeinschaft $=79$. Vgl. auch Grabitz $=$ Rechtsetzungsbefugnis $=10 \mathrm{ff}$. 


\section{Die Aufgabenverteilung}

Die Betrachtung des Finanzausgleichs innerhalb der Europäischen Gemeinschaften muß an der Frage der Aufgabenverteilung ansetzen. Die Aufgaben der Europäischen Gemeinschaften werden zunächst durch die Verträge umrissen.

\section{Die Aufgaben der Europäischen Gemeinschaft für Kohle und Stahl}

Mit dem Vertrag über die Gründung der Europäischen Gemeinschaft für Kohle und Stahl wurde ein wichtiger Teil der Industriepolitik dem nationalen Hoheitsbereich entzogen. Nach Art. 2 Abs. 1 EGKSV ist die Europäische Gemeinschaft für Kohle und Stahl dazu berufen, im Einklang mit der Gesamtwirtschaft der Mitgliedstaaten und auf der Grundlage eines Gemeinsamen Marktes, wie er in Artikel 4 näher bestimmt wird, zur Ausweitung der Wirtschaft, zur Steigerung der Beschäftigung und zur Hebung der Lebenshaltung in den Mitgliedstaaten beizutragen. Dabei soll gemäß Art. 3 EGKSV vor allem der technische Fortschritt in der Montanindustrie gefördert und eine Verbesserung der Lebens- und Arbeitsbedingungen der Arbeiter angestrebt werden.

Die Gemeinschaft soll informieren und beraten; sie kann Unternehmen Finanzmittel zur Verfügung stellen und sich an den Lasten der strukturellen Anpassung in den Sektoren Kohle und Stahl beteiligen; sie hat allerdings auch die Möglichkeit, direkt in den Markt einzugreifen, "wenn es die Umstände erfordern"1.

Als mit dem Gemeinsamen Markt nicht vereinbar erklärt wurden Ein- und Ausfuhrzölle, Abgaben gleicher Wirkung, mengenmäßige Beschränkungen des Warenverkehrs, diskriminierende Praktiken, Subventionen und Marktaufteilungen. ${ }^{2}$ Die Gewährung von Beihilfen für die Sektoren Kohle und Stahl sollten der Gemeinschaft vorbehalten bleiben. ${ }^{3}$

Die Europäische Gemeinschaft für Kohle und Stahl ist ihrer wettbewerbspolitischen Aufgabe in der Realität allerdings nur sehr unzureichend gerecht ge-

1 Vgl. Art. 5 EGKSV.

2 Vgl. Art. 4 EGKSV.

3 Vgl. Art. 54-56 EGKSV. 
worden. Durch die Schaffung eines Gemeinsamen Marktes verlagerte man lediglich bis dahin nationale Regulierungen und protektionistische Maßnahmen auf die Gemeinschaftsebene. Der Krise der Stahlindustrie setzten die Europäischen Gemeinschaften lange Zeit vor allem eine Kontingentierungspolitik entgegen. Inzwischen ist das 1980 eingeführte Produktionsquotensystem allerdings ausgelaufen. ${ }^{4}$ Nationale Subventionen wurden entgegen Art. 54-56 EGKSV weiter geduldet und lediglich bestimmten Vorgaben unterworfen. Die seit 1986 geltenden Regelungen lassen nur noch wenige Arten von Beihilfen zu und die Voraussetzungen für ihre Gewährung wurden verschärft. 5

Dennoch bestehen in der Bundesrepublik Deutschland beispielsweise umfangreiche nationale Beihilfen, insbesondere Absatzhilfen für den Kohleverkauf. So werden durch den sogenannten Jahrhundertvertrag die Unternehmen der Energieversorgung verpflichtet, ihre inländischen Steinkohlebezüge von 37 Mio. Tonnen 1981 um jährlich 1 Mio. Tonnen auf 47,5 Mio. Tonnen zu erhöhen. Die Mehrkosten, die durch die Verstromung inländischer Steinkohle gegenüber der Verstromung von schwerem Heizöl entstehen, werden über den "Kohlepfennig", der den Stromverbrauchern angelastet wird, gedeckt. Obwohl die Kommission die Bundesrepublik Deutschland aufgefordert hat, diese Ausgleichszahlungen allmählich abzubauen, wurden sie bisher kaum merklich reduziert. Durch die deutsche Kokskohlenbeihilfe wird der Preisunterschied zwischen inländischer und importierter Kokskohle ausgeglichen. Außerdem bestehen zum Schutz der heimischen Kohle Importkontingente. ${ }^{6}$ Auch für die Eisen- und Stahlindustrie in den neuen Ländern wurden zahlreiche Beihilfen von den Europäischen Gemeinschaften gebilligt. ${ }^{7}$ Der nationale Spielraum ist also bisher nicht allzusehr eingeschränkt.

\section{Die Aufgaben der Europäischen Atomgemeinschaft}

Seit dem 1.1.1958 ist der Vertrag zur Gründung der Europäischen Atomgemeinschaft in Kraft. Nach Art. 1 EAGV soll diese Gemeinschaft die für die schnelle Bildung und Entwicklung von Kernindustrien erforderlichen Voraus-

4 Vgl. Kommission der EG $=$ XXIII. Gesamtbericht $=154$.

5 Vgl. Kommission der EG =Beihilfen der Eisen- und Stahlindustrie 1985= 1ff.

6 Vgl. Sachverständigenrat zur Begutachtung der gesamtwirtschaftlichen Entwicklung $=$ Jahresgutachten $1989 / 90=$ Ziff. $325 \mathrm{f}$.

7 Vgl. Presse- und Informationsamt der Bundesregierung $=$ Einbeziehung $=15$. 
setzungen schaffen und so zur Hebung der Lebenshaltung in den Mitgliedstaaten und zur Weiterentwicklung der Beziehungen mit den anderen Staaten beitragen.

Für die Erfüllung dieser Aufgabe hat die Gemeinschaft vor allem die Forschung zu entwickeln und die Verbreitung technischer Kenntnisse sicherzustellen. ${ }^{8}$ So fördert sie ein Forschungs- und Ausbildungsprogramm zum Strahlenschutz; ${ }^{9}$ weitere gemeinschaftliche Forschungsprogramme befassen sich mit der kontrollierten Kernfusion ${ }^{10}$ und der Bewirtschaftung und Lagerung radioaktiver Abfälle ${ }^{11}$. Im Rahmen der Gemeinsamen Forschungsstelle der Europäischen Gemeinschaften ${ }^{12}$ sind vor allem die vier Institute für Sicherheitstechnologie, Transurane, fortgeschrittene Werkstoffe und Umwelt für die Forschung der Europäischen Atomgemeinschaft zuständig.

Neben den Forschungsaufgaben hat die Europäische Atomgemeinschaft auch den Auftrag, Sicherheitsnormen für den Gesundheitsschutz der Bevölkerung und der Arbeitskräfte aufzustellen und für ihre Anwendung zu sorgen. ${ }^{13}$ Beispiele hierfür sind die Festlegung von Grundnormen für den Strahlenschutz im Hinblick auf einen optimalen Schutz der betroffenen Arbeitskräfte ${ }^{14}$ und die Festlegung von Höchstwerten für die Radioaktivität in Nahrungs- und Futtermitteln im Falle eines nuklearen Unfalls oder einer anderen radiologischen Notsituation 15 .

Die Gemeinschaft soll außerdem Investitionen im Bereich der Kernenergie erleichtern ${ }^{16}$ und für eine regelmäßige und gerechte Versorgung aller Benutzer der Gemeinschaft mit Erzen und Brennstoffen Sorge tragen ${ }^{17}$. Ferner hat sie

8 Vgl. Art. 2 Buchstabe a) EAGV.

9 Vgl. Rat der EG =Strahlenschutzprogramm 1990-1991 = 50ff.

10 Vgl. Rat der EG $=$ Kontrollierte Kernfusion $1988=5 \mathrm{ff}$.

11 Vgl. Kommission der EG =XXIII. Gesamtbericht $=170 \mathrm{f}$.

12 Die Gemeinsame Forschungsstelle umfabt neun Institute in den Standorten Ispra (Italien), Geel (Belgien), Karlsruhe (Bundesrepublik Deutschland) und Petten (Niederlande), vgl. Kommission der EG $=$ XXIII. Gesamtbericht $=158$.

13 Vgl. Art. 2 Buchstabe b) EAGV.

14 Vgl. Rat der EG =Richtlinie zum Strahlenschutz 1980=1ff.

15 Vgl. Rat der EG =Höchstwerte an Radioaktivität $1989=1 \mathrm{ff}$.

16 Vgl. Art. 2 Buchstabe c) EAGV.

17 Vgl. Art. 2 Buchstabe d) EAGV. 
darüber zu wachen, daß die Kernstoffe nicht anderen als den vorgesehenen Zwecken zugeführt werden. 18

\section{Die Aufgaben der Europäischen Wirtschaftsgemeinschaft}

Während die Europäische Gemeinschaft für Kohle und Stahl und die Europäische Atomgemeinschaft sektoral begrenzt sind, ist der Vertrag zur Gründung der Europäischen Wirtschaftsgemeinschaft umfassender angelegt. Die allgemeinen Ziele der Gemeinschaft sind in Art. 2 EWGV festgelegt. Danach soll die Gemeinschaft durch die Errichtung des Gemeinsamen Marktes und die schrittweise Annäherung der Wirtschaftspolitik eine harmonische Entwicklung des Wirtschaftslebens, eine beständige und ausgewogene Wirtschaftsausweitung und eine größere Stabilität, eine beschleunigte Hebung der Lebenshaltung und engere Beziehungen zwischen den Staaten fördern. Für welche konkreten Politikbereiche der Gemeinschaft dabei Zuständigkeiten zufallen, wird durch den Vertrag zur Gründung der Europäischen Wirtschaftsgemeinschaft festgelegt, denn es gilt grundsätzlich das Prinzip der enumerativen Einzelermächtigung. Gemäß Art. 189 Abs. 1 EWGV werden die Rechtsetzungsorgane der Europäischen Gemeinschaften "nach Maßgabe dieses Vertrages" zum Erlaß von Rechtsakten ermächtigt, d. h. die Europäischen Gemeinschaften sind nur dann für bestimmte Aufgaben zuständig, wenn die Verträge ausdrücklich $\mathrm{Zu}$ ständigkeiten von Gemeinschaftsorganen für entsprechende Maßnahmen vorsehen. ${ }^{19}$ Die Bereiche, in denen sich eine Politik der Gemeinschaft entwickeln soll, werden allerdings nur in sehr groben Umrissen durch die Verträge abgesteckt. Es bleibt ein sehr großer Gestaltungsspielraum sowohl für den Bereich der aufgegriffenen Aufgaben als auch für die Intensität der Aufgabenerfüllung. 20

Im ersten Teil des EWG-Vertrages werden die allgemeinen Grundsätze der Gemeinschaft festgelegt. Neben den Zielen des Art. 2 EWGV werden in Art. 3

18 Vgl. Art. 2 Buchstabe e) EAGV. Im Rahmen der Sicherheitsüberwachung der EAG wurden 1989 Buchungs- und materielle Kontrollen über Durchschnittsbestände von ca. $160 \mathrm{t}$ Plutonium, $12 \mathrm{t}$ hochangereichertem Uran, $188.000 \mathrm{t}$ Uran, Thorium und Schwerwasser durchgeführt. Dieses Material war auf 750 kerntechnische Anlagen der Gemeinschaft verteilt. Die Betreiber haben etwa 410.000 Meldezettel über die Materialbewegungen und Bestände übermittelt. Vgl. Kommission der EG =XXIII. Gesamtbericht $=312$.

19 Vgl. dazu auch Beutler u. a. =Europäische Gemeinschaft $=75 \mathrm{f}$.

20 Vgl. Andel =Europäische Gemeinschaften $=313$. 
EWGV Politikbereiche genannt, in denen die Gemeinschaft zunächst tätig werden durfte. Dies waren:

a) die Abschaffung der Zölle und mengenmäßigen Beschränkungen bei der Ein- und Ausfuhr von Waren sowie aller sonstigen Maßnahmen gleicher Wirkung zwischen den Mitgliedstaaten;

b) die Einführung eines gemeinsamen Zolltarifs und einer gemeinsamen Handelspolitik gegenüber Drittländern;

c) die Beseitigung der Hindernisse für den freien Personen-, Dienstleistungsund Kapitalverkehr zwischen den Mitgliedstaaten;

d) die Einführung einer gemeinsamen Politik auf dem Gebiet der Landwirtschaft;

e) die Einführung einer gemeinsamen Politik auf dem Gebiet des Verkehrs;

f) die Errichtung eines Systems, das den Wettbewerb innerhalb des Gemeinsamen Marktes vor Verfälschungen schützt;

g) die Anwendung von Verfahren, welche die Koordinierung der Wirtschaftspolitik der Mitgliedstaaten und die Behebung von Störungen im Gleichgewicht ihrer Zahlungsbilanzen ermöglichen;

h) die Angleichung der innerstaatlichen Rechtsvorschriften, soweit dies für das ordnungsgemäße Funktionieren des Gemeinsamen Marktes erforderlich ist;

i) die Schaffung eines Europäischen Sozialfonds, um die Beschäftigungsmöglichkeiten der Arbeitnehmer zu verbessern und zur Hebung ihrer Lebenshaltung beizutragen;

j) die Errichtung einer Europäischen Investitionsbank, um durch Erschließung neuer Hilfsquellen die wirtschaftliche Ausweitung in der Gemeinschaft zu erleichtern;

k) die Assoziierung der überseeischen Länder und Hoheitsgebiete, um den Handelsverkehr zu steigern und die wirtschaftliche und soziale Entwicklung durch gemeinsame Bemühungen zu fördern.

Den allgemeinen Grundsätzen wurde durch die Einheitliche Europäische Akte vom Februar 1986 der Art. 8a EWGV hinzugefügt, der die Verwirklichung des Binnenmarktes bis zum 31.12.1992 vorsieht. Dieser Binnenmarkt soll ein Raum ohne Binnengrenzen sein, in dem der freie Verkehr von Waren, Personen, Dienstleistungen und Kapital gewährleistet ist.

Der zweite Teil des Vertrages zur Gründung der Europäischen Wirtschaftsgemeinschaft ("Grundlagen der Gemeinschaft") enthält Regelungen über den 


\section{Aufgabenverteilung}

freien Warenverkehr, über die Freizügigkeit und über den freien Dienstleistungs- und Kapitalverkehr. Diese Aufgaben der Europäischen Gemeinschaften sind vor allem ordnungspolitischer Natur. Im zweiten Teil werden darüber hinaus jedoch auch schon die Umrisse zweier wichtiger Bereiche der Gemeinschaftspolitik skizziert, nämlich der gemeinsamen Agrarpolitik und der gemeinsamen Verkehrspolitik.

Im dritten Teil des Vertrages zur Gründung der Europäischen Wirtschaftsgemeinschaft ("Die Politik der Gemeinschaft") werden zunächst als "Gemeinsame Regeln" Wettbewerbsregeln, steuerliche Vorschriften und Regelungen zur Angleichung von Rechtsvorschriften festgelegt. Ein weiterer Titel beschäftigt sich mit der wirtschaftlichen Zusammenarbeit, der Konjunkturpolitik, der Zahlungsbilanz und der Handelspolitik. In Titel III ("Sozialpolitik") werden die Mitgliedstaaten aufgefordert, ihre Sozialordnungen aufeinander abzustimmen und die Zusammenarbeit in sozialen Fragen voranzutreiben. Außerdem werden Grundsätze für die Errichtung, den Zweck und die Arbeit eines Europäischen Sozialfonds aufgestellt. Titel IV enthält genauere Regelungen für die Errichtung und die Aufgaben einer Europäischen Investitionsbank. Erst im Jahre 1987 sind durch die Einheitliche Europäische Akte die letzten drei Titel des Vertrages zur Gründung der Europäischen Wirtschaftsgemeinschaft - "Wirtschaftlicher und sozialer Zusammenhalt", "Forschung und technologische Entwicklung" sowie "Umwelt" - hinzugekommen.

Die wichtigsten Aufgabenbereiche der Europäischen Wirtschaftsgemeinschaft sollen im folgenden genauer betrachtet werden.

\section{a) Errichtung eines Gemeinsamen Marktes}

Das wichtigste Ziel der Gemeinschaft ist die Errichtung eines Gemeinsamen Marktes auf der Basis eines möglichst verzerrungsfreien Wettbewerbs. Darauf zielen insbesondere die unter den Buchstaben a, b, c, f und h des Art. 3 EWGV aufgeführten Tätigkeiten ab.

Beim tarifären Außenhandel wird häufig von einer ausschließlichen Kompetenz $\mathrm{z}^{21}$ der Gemeinschaft gesprochen. ${ }^{22}$ Obwohl eine Zollunion mit Zollfreiheit

21 "'Ausschließliche Kompetenzen' bedeutet, daß nur mehr die EG zuständig ist, die Mitgliedstaaten also zur Rechtsetzung nicht weiter befugt sind." Schweitzer = Bundesstaat und $\mathrm{EG}=18$. 
im Binnenhandel und gemeinsamem Außenzolltarif größtenteils verwirklicht ist, haben die Mitgliedstaaten jedoch noch immer bestimmte Einflußmöglichkeiten. ${ }^{23}$ So sieht Art. 115 EWGV die Möglichkeit nationaler handelspolitischer Schutzmaßnahmen nach Einholung der notwendigen Ermächtigung durch die Kommission vor. Bisher nutzten die Mitgliedstaaten auch noch rege das Instrumentarium der nicht-tarifären Handelshemmnisse. Diese sollen allerdings bis zur Vollendung des Binnenmarktes weiter vereinheitlicht oder ganz abgeschafft werden.

Auch die Gestaltung der vertraglichen Außenhandelspolitik fällt mittlerweile zu großen Teilen in die Zuständigkeit der Gemeinschaft - etwa Verhandlungen über den Abschluß von Handels- und Kooperationsabkommen mit Drittstaaten, von Abkommen auf multilateraler Ebene (verschiedene Rohstoffabkommen) sowie Verhandlungen mit Organen oder Vertretungen außereuropäischer regionaler Wirtschaftsintegrationen. Allerdings bleibt auch hier den Mitgliedstaaten noch Gestaltungsspielraum für nationalstaatliche, bilaterale Maßnahmen, beispielsweise bei der vertraglichen Sicherung der Rahmenbedingungen von Auslandsinvestitionen durch bilaterale Investitionsschutzabkommen oder Doppelbesteuerungsabkommen. ${ }^{24}$

Neben dem gemeinschaftlichen Außenhandel tritt nun verstärkt die Verwirklichung des Binnenmarktes gemäß Art. 8a EWGV in den Vordergrund. Im Juni 1985 legte die Kommission der Europäischen Gemeinschaften ein "Weißbuch zur Vollendung des Binnenmarktes"25 vor, das zahlreiche Vorschläge zur Beseitigung materieller Schranken (wie die durch Grenzkontrollen verursachten Behinderungen und Kosten), technischer Schranken (z. B. durch unterschiedliche Normen, Vorschriften, Zulassungsverfahren und national abgeschottete Märkte im öffentlichen Beschaffungswesen) und Steuerschranken macht. Es geht dabei vor allem um die Beseitigung der Grenzkontrollen, den Abbau von technischen Handelshemmnissen, die Liberalisierung der Dienstleistungen und der Kapitalmärkte, die Öffnung der staatlichen Beschaffungs-

22 Vgl. Beutler u. a. = Europäische Gemeinschaft= 532 .

23 Besondere Regelungen gelten für die neuen Länder. So können in diesen für bestimmte Produkte aus Polen, Ungarn, der Tschechoslowakei, Rumänien, Bulgarien, der UdSSR und Jugoslawien Zollbefreiungen gewährt werden. Vgl. dazu Presse- und Informationsamt der Bundesregierung =Einbeziehung $=2 \mathrm{ff}$.

24 Vgl. Hackenbroch $=$ Supranationaler Finanzausgleich $=49$.

25 Kommission der EG = Vollendung des Binnenmarktes=. 
märkte und die Angleichung der Umsatzsteuer und sonstiger Verbrauchsteuern. ${ }^{26}$ Ein Teil der Kommissionsvorschläge wurde inzwischen verwirklicht; wichtige Fragen, etwa der Abbau von Steuergrenzen, sind jedoch nicht oder noch nicht endgültig geklärt. 27

Die Gemeinschaft versucht, den Binnenmarkt vor allem mit dem Instrument der Harmonisierung von Rechtsvorschriften zu realisieren. Diese Harmonisierung war im Vertrag zur Gründung der Europäischen Wirtschaftsgemeinschaft von vornherein vorgesehen (Art. 3h EWGV). In Verbindung mit den Art. 100ff. EWGV eröffnet dies der Gemeinschaft die Möglichkeit, auf viele Politikbereiche Einfluß zu nehmen, die nach den Verträgen eigentlich nicht zu ihrem Aufgabengebiet gehören. Nach der Generalklausel in Art. 100 EWGV können Vorschriften aus allen Rechtsgebieten harmonisiert werden, soweit sich diese unmittelbar auf das Errichten oder Funktionieren des Gemeinsamen Marktes beziehen. Über diese Regelung wurden beispielsweise einige Umweltrichtlinien verabschiedet, als die Umweltpolitik noch nicht als Aufgabe in den Verträgen verankert war. ${ }^{28}$ Weitere wichtige Beispiele für die Erweiterung der Kompetenzen durch die Harmonisierung von Rechtsvorschriften im Hinblick auf den Binnenmarkt sind die Medienpolitik und die Sozialpolitik. Für diese Bereiche besitzen die Europäischen Gemeinschaften keine vertraglichen $\mathrm{Zu}$ ständigkeiten. ${ }^{29}$ Dennoch hat die Gemeinschaft Kompetenzen an sich gezogen, z. B. durch die Fernsehrichtlinie ${ }^{\mathbf{3 0}}$ oder das Aktionsprogramm zur Anwendung der sozialen Grundrechte ${ }^{31}$.

Der Einsatz des Art. 100 EWGV ist immer nur auf schon bestehende oder geplante nationale Rechtsvorschriften beschränkt. Darüber hinausgehende gestalterische Möglichkeiten eröffnet dagegen Art. 235 EWGV. Dieser Artikel

26 Vgl. dazu auch Hermann, Ochel, Wegner =Auswirkungen= 14 und Dichtl =Europäischer Binnenmarkt $=333 \mathrm{ff}$.

27 Vgl. dazu u. a. Sachverständigenrat zur Begutachtung der gesamtwirtschaftlichen Entwicklung = Jahresgutachten 1990/91 = Ziff. 462ff. und Presse- und Informationsamt der Bundesregierung $=$ Stand und Aussichten $=1 \mathrm{ff}$.

28 Vgl. Bungarten $=$ Umweltpolitik $=186$.

29 Im Vertrag zur Gründung der Europäischen Wirtschaftsgemeinschaft gibt es zwar einen eigenen Titel "Sozialpolitik", doch enthält dieser keine umfassende Regelung der Sozialpolitik. Der Vertrag beschränkt sich darauf, eine Zusammenarbeit der Mitgliedstaaten herbeizuführen und sozialpolitische Teilaspekte zu regeln. Vgl. dazu Beutler u. a. =Europäische Gemeinschaft $=436$.

30 Vgl. Rat der EG $=$ Fernsehrichtlinie $1989=23 \mathrm{ff}$.

31 Vgl. Kommission der EG = Soziale Grundrechte $=$. 
sieht vor, daß der Rat durch einstimmigen Beschluß - auf Vorschlag der Kommission - geeignete Vorschriften erlassen kann, wenn ein Tätigwerden der Gemeinschaft erforderlich scheint, um im Rahmen des Gemeinsamen Marktes eines ihrer Ziele zu verwirklichen und im Vertrag die dazu erforderlichen Befugnisse nicht vorhanden sind. Diese Regelung ist zunehmend " ... als Grundlage für Maßnahmen im Rahmen nur unvollständig im Vertrag geregelter, sog. 'flankierender' Politiken, herangezogen worden ..."32. Im Grunde führt die "Vertragslückenschließung" durch Art. 235 EWGV zu einer Durchbrechung des Prinzips der begrenzten Einzelermächtigung. ${ }^{33}$

Buchstabe f des Art. 3 EWGV weist auf die wettbewerbspolitischen Befugnisse der Europäischen Wirtschaftsgemeinschaft hin, die im dritten Teil des Vertrages genauer geregelt werden. Art. 85-90 EWGV enthalten Vorschriften für Unternehmen, z. B. das Verbot wettbewerbsbehindernder Vereinbarungen oder das Verbot des Mißbrauchs einer den Markt beherrschenden Stellung. Für die Kompetenzverteilung zwischen den Europäischen Gemeinschaften und den Mitgliedstaaten besonders bedeutsam sind die Regelungen für staatliche Beihilfen nach Art. 92-94 EWGV. Die Gemeinschaft hat das Recht, nationale Beihilfen zu prüfen und für den Fall, daß eine Beihilfe mit dem Gemeinsamen Markt unvereinbar ist oder mißbräuchlich angewandt wird, den betreffenden Staat zu verpflichten, die Beihilfe aufzuheben oder umzugestalten. Wird dieser Entscheidung nicht Folge geleistet, kann die Gemeinschaft unmittelbar den Europäischen Gerichtshof anrufen. Die Gemeinschaft hat damit für nationale Beihilfen eine Aufsichts-, Beurteilungs- und Entscheidungskompetenz, wobei ihr ein weiter Ermessensspielraum eingeräumt wird. ${ }^{34}$ Diese Befugnis gibt der Gemeinschaft die Möglichkeit, auf die Bereiche der nationalen Politik, die mit Fördermaßnahmen verbunden sind - wie z. B. die Technologiepolitik oder die Regionalpolitik - Einfluß zu nehmen.

Trotz dieses Aufsichtsrechtes ist das Niveau der Beihilfen in den Mitgliedstaaten relativ hoch. ${ }^{35}$ Es hat den Anschein, daß die nationalen Regierungen ihren Einfluß auf die wirtschaftliche Entwicklung verstärkt mit (versteckten) Beihilfen zu erhalten versuchen, nachdem sie im Zusammenhang mit der erforder-

32 Beutler u. a. $=$ Europäische Gemeinschaft $=77 \mathrm{f}$.

33 Vgl. Schweitzer $=$ Bundesstaat und $E G=15 f$.

34 Vgl. Matthies $=$ Subventionsrecht $=446$.

35 Vgl. Kommission der EG $=$ Staatliche Beihilfen $\mathrm{II}=1$. 
lichen Harmonisierung im Rahmen der Verwirklichung des Binnenmarktes wirtschaftspolitische Souveränität eingebüßt haben. ${ }^{36}$

\section{b) Gemeinsame Landwirtschaftspolitik}

Art. 3 Buchstabe d EWGV fordert die Einrichtung einer gemeinsamen Landwirtschaftspolitik. Die Bedeutung dieser sektoralen Politik wird dadurch deutlich, daß ihr im zweiten Teil des Vertrages zur Gründung der Europäischen Wirtschaftsgemeinschaft ein eigener Titel eingeräumt wurde. Die Agrarpolitik verfolgt nach Art. 39 Satz 1 EWGV das Ziel, die Produktion der Landwirtschaft durch die Förderung des technischen Fortschritts, die Rationalisierung der landwirtschaftlichen Erzeugung und den bestmöglichen Einsatz der Produktionsfaktoren zu steigern. Der landwirtschaftlichen Bevölkerung soll - insbesondere durch die Erhöhung des Pro-Kopf-Einkommens der in der Landwirtschaft tätigen Personen - eine angemessene Lebenshaltung gewährleistet werden; die Agrarmärkte sollen stabilisiert und die Versorgung sichergestellt werden. Außerdem ist für die Belieferung der Verbraucher zu angemessenen Preisen Sorge zu tragen. Aus diesem Zielbündel ist das Ziel der Einkommenssicherung der Landwirte im Laufe der Zeit immer mehr in den Vordergrund getreten. 37

Auch die Agrarpolitik wird häufig zu den ausschließlichen Kompetenzen der Europäischen Gemeinschaften gerechnet. Dies ist jedoch nur für die Agrarmarktpolitik korrekt. ${ }^{38}$ Die Agrarmarktpolitik besteht aus Preisgarantiesystemen für eine große Anzahl von Agrarprodukten, wobei die Gemeinschaft die von ihr fixierten Mindestpreise durch staatlichen Ankauf und staatliche Vorratshaltung im Binnenhandel und durch variable Exporterstattungen und Abschöpfungen im Außenhandel verwirklicht. Auf die Probleme, die die Agrarpolitik seit langem aufwirft, soll erst an späterer Stelle näher eingegangen werden. ${ }^{39}$ Doch haben Reformüberlegungen inzwischen dazu geführt, daß

36 Vgl. dazu Sachverständigenrat zur Begutachtung der gesamtwirtschaftlichen Entwicklung = Jahresgutachten 1990/91 = Ziff. 467.

37 Vgl. dazu vor allem Petersen $=$ Agrarpolitik $=51$. $\mathrm{Zu}$ den volkswirtschaftlichen Kosten der Versuche, durch Marktinterventionen das Pro-Kopf-Einkommen in der Landwirtschaft zu erhöhen, vgl. Schrader $=$ Agrar- und Haushaltspolitik $=3 \mathrm{ff}$.

38 Vgl. Hackenbroch $=$ Supranationaler Finanzausgleich $=47$ und EuGH Rs. 63/193, Slg. 1984, S. 2689.

39 Vgl. dazu Abschnitt D. I. 3. b) und D. III. 2. 
die Agrarmarktpolitik der Europäischen Gemeinschaften durch ein System von sogenannten "Stabilisatoren" ergänzt wurde, durch das die Garantiepreise bei wichtigen Marktordnungsprodukten auf bestimmte Mengen begrenzt werden und ein Überschreiten festgelegter Garantiemengen eine automatische $\mathrm{Ab}$ senkung der Stützpreise zur Folge hat. ${ }^{\mathbf{4 0}}$

Neben der Agrarmarktpolitik wird die Gemeinschaft auch bei der Agrarstrukturpolitik tätig. Die gemeinschaftliche Agrarstrukturpolitik ist allerdings nur eine Ergänzung nationaler Maßnahmen, die in der Bundesrepublik Deutschland sowohl vom Bund als auch von den Ländern - vor allem im Rahmen der Gemeinschaftsaufgabe "Verbesserung der Agrarstruktur und des Küstenschutzes"41 - getroffen werden. Man spricht daher bei der Agrarstrukturpolitik von einer konkurrierenden Kompetenz der Europäischen Gemeinschaften. Auch die Agrarsozialpolitik - als weitere ergänzende Politik - ist trotz der Tätigkeit des Europäischen Sozialfonds immer noch eine Domäne der nationalen Politik, in der Bundesrepublik vor allem des Bundes. Auch auf große Teile des für den Agrarsektor relevanten Steuerrechts haben die Europäischen Gemeinschaften bisher keinen Einfluß. ${ }^{42}$

\section{c) Gemeinsame Verkehrspolitik}

Der Errichtung einer gemeinsamen Verkehrspolitik wird im zweiten Teil des Vertrages zur Gründung der Europäischen Wirtschaftsgemeinschaft ebenfalls ein eigener Titel eingeräumt. Die Gemeinschaft soll gemeinsame Regeln für den grenzüberschreitenden Verkehr aufstellen und die Bedingungen für die Zulassung von Unternehmen zum Verkehr innerhalb eines Mitgliedstaates, in

40 Vgl. Kommission der EG =Lage der Landwirtschaft 1988= 15.

41 Nach Art. 91a GG wirkt der Bund in diesem Bereich bei der Erfüllung von Aufgaben der Länder mit, wenn diese Aufgaben für die Gesamtheit bedeutend sind und die Mitwirkung des Bundes zur Verbesserung der Lebensverhältnisse erforderlich ist. Der Bund beteiligt sich an der Rahmenplanung. Es existiert ein Planungsausschuß, der einen mittelfristigen (auf 4 Jahre konzipierten) Rahmenplan aufstellt. Daran beteiligt sind der zuständige Fachminister des Bundes, der Bundesminister der Finanzen und je ein Minister eines Landes. Die Stimmenzahl des Bundes entspricht der Zahl der Länder. Beschlüsse bedürfen einer 3/4-Mehrheit der Stimmen. Vgl. Frey =Finanzverfassung $=38$. Der Rahmenplan setzt Rahmen- und Eckdaten und legt die Leitlinien der Förderung fest. Die konkrete Ausgestaltung und Durchführung des Rahmenplans bleibt in der Regel Aufgabe der Länder. Vgl. Kaiser = Regionalpolitik $=176$.

Vgl. Scheper $=$ Gemeinsame Agrarpolitik $=108$. 
dem sie nicht ansässig sind (sogenannte Kabotage), festlegen. ${ }^{43}$ Es geht hauptsächlich darum, Wettbewerbsverzerrungen im Gemeinsamen Markt als Folge unterschiedlicher nationaler Vorschriften der Verkehrsordnungen zu beseitigen. Durch den diskriminierungsfreien Zugang der Verkehrsunternehmen aller Mitgliedstaaten zum grenzüberschreitenden Verkehr sowie die Zulassung zur Kabotage soll die Dienstleistungsfreiheit auch im Bereich des Verkehrs verwirklicht werden. ${ }^{44}$ Ein weiteres Ziel ist die Erhöhung der Verkehrssicherheit.

Der Rat hat die Ermächtigung des Vertrages zur Gründung der Europäischen Wirtschaftsgemeinschaft für die Verkehrspolitik zunächst nur sehr zögernd und punktuell genutzt. ${ }^{45}$ Schließlich hat der Europäische Gerichtshof am 22.5.1985 die Verwirklichung des Vertragsziels der gemeinsamen Verkehrspolitik in angemessener Frist in seinem "Untätigkeitsurteil" angemahnt. ${ }^{46}$ Inzwischen haben die Europäischen Gemeinschaften im Rahmen der Maßnahmen zur Vollendung des Binnenmarktes ihre Aufgabenkompetenz im Bereich der Verkehrspolitik stärker wahrgenommen, etwa durch die Festlegung gemeinsamer Sozialvorschriften (Ruhezeiten für Personen- und Güterbeförderung) ${ }^{47}$, die Festlegung einheitlicher beruflicher und finanzieller Voraussetzungen für grenzüberschreitende Verkehrsunternehmer ${ }^{48}$ und durch die Kabotage-Regelung, nach der seit dem 1.7.1990 Straßenverkehrsunternehmen aus den Europäischen Gemeinschaften in begrenztem Umfang Güter innerstaatlich in anderen Mitgliedstaaten transportieren dürfen, ohne daß sie dort über Sitz oder Niederlassung verfügen. Dazu wird eine begrenzte Anzahl (15.000) von befristeten Genehmigungen erteilt, deren Zahl jährlich um $10 \%$ aufgestockt werden soll. 49

43 Vgl. Art. 75 EWGV.

44. Vgl. Eickhof, Berkelova $=$ Verkehrspolitik $=597$.

45 Vgl. Lenz $=$ Verkehrspolitik der $E G=160$.

46 EuGH 22.5.1985, Rs. 13/83 (Parlament ./. Rat), Slg. (1985) $1513=$ EuR (1985) 393 mit Besprechung.

47 Vgl. Rat der EG =Sozialvorschriften im Straßenverkehr 1985=1ff. Die Vorschriften werden allerdings nicht in allen Mitgliedstaaten gleich sorgfältig überwacht.

48 Dabei sollen sämtliche Unternehmer ohne weitere fachliche Prüfung eine Zulassung für den grenzüberschreitenden Transport erhalten, wenn sie eine fünfjährige Berufserfahrung aufweisen können. Auch für das Eigenkapital wurden Mindestregelungen 150 ECU pro Tonne oder mindestens 3.000 ECU je Fahrzeug - festgelegt, vgl. o. V. $=$ Liberalisierter Verkehrsmarkt $=17$.

49 Vgl. Eickhof, Berkelova $=$ Verkehrspolitik $=597$. 
Neben den Maßnahmen zur Vollendung des Binnenmarktes sind die Europäischen Gemeinschaften zunehmend als Partner bei Verhandlungen mit Nachbarstaaten (z. B. Österreich) über den Transitverkehr tätig geworden. ${ }^{50}$ Außerdem beginnt sich in den Europäischen Gemeinschaften die Auffassung durchzusetzen, daß sie auch der Verkehrsinfrastruktur ihre Aufmerksamkeit zuwenden sollten. ${ }^{51}$ Die Ansätze hierfür sind jedoch bisher bescheiden.

\section{d) Koordinierung der Wirtschaftspolitik}

Die wesentlichen wirtschaftspolitischen Aufgaben sind grundsätzlich den Mitgliedstaaten vorbehalten. Art. 3 Buchstabe g EWGV sieht allerdings die Anwendung von Verfahren vor, welche die Koordinierung der Wirtschaftspolitik und die Behebung von Störungen im Gleichgewicht der Zahlungsbilanzen der Mitgliedstaaten ermöglichen. Die Zusammenarbeit in der Wirtschafts- und Währungspolitik wird in Art. 102aff. EWGV näher geregelt. Um die für die Weiterentwicklung der Gemeinschaft erforderliche Konvergenz zu erreichen, sollen die Mitgliedstaaten gemäß den Zielen des Art. 104 EWGV zusammenarbeiten. Als Ziele der nationalen Wirtschaftspolitik nennt Art. 104 EWGV die Wahrung eines hohen Beschäftigungsstandes und eines stabilen Preisniveaus, die Sicherung des Gleichgewichts der Zahlungsbilanz und die Aufrechterhaltung des Vertrauens in die Währung. In neuerer Zeit wurde dabei dem Ziel der Preisniveaustabilität eine größere Bedeutung eingeräumt als früher. ${ }^{\mathbf{5 2}}$

Bei der Zusammenarbeit sind die Erfahrungen, die im Rahmen des Europäischen Währungssystems gesammelt wurden, zu berücksichtigen: Durch die Errichtung dieses Währungsverbundes sollte die währungspolitische Zusammenarbeit verstärkt werden mit dem Ziel, eine Zone der währungspolitischen Stabilität in Europa zu schaffen. Eine zentrale Stellung nimmt dabei der ECU $^{53}$ ein, in den die Währungen aller Mitgliedstaaten mit festen Beträgen eingehen. Die einzelnen Währungen sind über den ECU als Bezugsgröße untereinander verbunden. Für jede nationale Währung wird ein in ECU ausgedrückter Leitkurs festgelegt. Über diese Leitkurse wird für die am

50 Vgl. z. B. o. V. =EG-Verkehrsministerrat $=17$.

51 Vgl. Beutler u. a. $=$ Europäische Gemeinschaft $=470$.

52 Vgl. Hasse $=$ Zweite Stufe $=342$.

53 European Currency Unit. 
Interventionsmechanismus teilnehmenden Währungen ${ }^{\mathbf{5 4}}$ ein Gitter bilateraler Leitkurse abgeleitet. Diese bilateralen Leitkurse fungieren bei der Wechselkursstabilierung als Mittelkurse. Die betreffenden Währungen dürfen nur innerhalb einer gewissen Bandbreite um die bilateralen Leitkurse schwanken. Für die Währungen Frankreichs, der Bundesrepublik Deutschland, der Niederlande, Belgiens, Luxemburgs, Dänemarks, Italiens und Irlands beträgt die Bandbreite 2,25\%, der spanischen und der britischen Währung wurde eine Bandbreite von $6 \%$ eingeräumt. Die zur Einhaltung der vereinbarten Schwankungsbreite erforderlichen Interventionen erfolgen zweiseitig: Erreicht etwa der Kurs der D-Mark gegenüber dem niederländischen Gulden den unteren Interventionspunkt, dann muß die Bundesbank Gulden nachfragen und die Niederländische Bank D-Mark anbieten. ${ }^{55}$ Bei nachhaltigen Spannungen, z. B. aufgrund unterschiedlicher Inflationsentwicklungen, können Wechselkursanpassungen durch die Änderung der Leitkurse vorgenommen werden.

Die Koordinationsbemühungen konzentrierten sich zunächst mit dem Europäischen Währungssystem auf den monetären Bereich. Dabei kann festgestellt werden, daß das Europäische Währungssystem bedingt erfolgreich war. Zwar verringerten sich die Wechselkursschwankungen, und die Inflationsraten näherten sich auf einem vergleichsweise niedrigen Niveau an. ${ }^{56}$ Doch erhielt seit Anfang der achtziger Jahre die Bekämpfung der Inflation weltweit Priorität und die Teilnehmer am Wechselkursmechanismus des Europäischen Währungssystems waren hier nicht erfolgreicher als die Gruppe der Industrieländer insgesamt. ${ }^{57}$ Die relative Konstanz der Wechselkurse konnte in einigen Fällen nur durch eine Ausweitung der Zinsdifferenz erreicht werden. Dadurch ist teilweise ein Anpassungsstau entstanden, der schließlich doch Realignments erzwingen kann. ${ }^{58}$ Außerdem ist die Finanzpolitik in den einzelnen Mitgliedstaaten nach wie vor durch starke Divergenzen gekennzeichnet.

Weitere Fortschritte in der Koordinierung der Wirtschaftspolitik hängen eng mit der Entwicklung der Gemeinschaft zur Wirtschafts- und Währungsunion

54 Dies sind inzwischen alle Währungen der Mitgliedstaaten mit Ausnahme von Portugal und Griechenland.

55 Vgl. Jarchow $=$ Geldmarkt $=67$.

56 Vgl. dazu auch Issing = Europäische Währungsintegration $=115 \mathrm{ff}$.

57 Vgl. Issing = Europäische Währungsintegration $=117$.

58 Vgl. dazu Sachverständigenrat zur Begutachtung der gesamtwirtschaftlichen Entwicklung = Jahresgutachten $1989 / 90=$ Ziff. 399 . 
zusammen. Die erste Phase der Wirtschafts- und Währungsunion, die am 1.7.1990 in Kraft getreten ist, wird charakterisiert durch den Beschluß des Rates über die Zusammenarbeit zwischen den Zentralbanken der Mitgliedstaaten der Europäischen Gemeinschaften ${ }^{59}$ und den Beschluß zur Erreichung einer schrittweisen Konvergenz der Politik und der wirtschaftlichen Ergebnisse während der ersten Stufe der Wirtschafts- und Währungsunion ${ }^{60}$.

Durch den ersten Beschluß erhält der Ausschuß der Zentralbankpräsidenten, der später in die Rolle einer Europäischen Zentralbank hineinwachsen soll, bestimmte Einflußmöglichkeiten. So ist er zu Entscheidungen der nationalen Zentralbanken über die Ausrichtung der Geldpolitik (etwa die jährliche Festlegung nationaler Geldmengenziele und Kreditmengenziele) normalerweise im voraus zu hören. Außerdem kann er Stellungnahmen an die einzelnen nationalen Regierungen und an den Ministerrat zu Maßnahmen abgeben, die die innere oder äußere monetäre Situation in der Gemeinschaft beeinflussen können. Schließlich gibt er - analog zu den Geschäftsberichten der nationalen Notenbanken - einen jährlichen Bericht über die monetäre und finanzielle Situation der Gemeinschaft heraus. ${ }^{61}$

Der tatsächliche Einfluß des Ausschusses der Zentralbankpräsidenten sollte nicht überschätzt werden. Bisher haben die nationalen Entscheidungsträger noch viele Möglichkeiten, sich dem Koordinationsprozeß zu entziehen. ${ }^{62}$ So ist der Ausschuß zwar normalerweise vor nationalen Entscheidungen zu hören. Was "normalerweise" heißt, bleibt allerdings offen. Weiter muß berücksichtigt werden, daß die Mehrzahl der Mitglieder des Ausschusses politisch nicht unabhängig, sondern an Weisungen der nationalen Regierungen gebunden ist. Schließlich können sich für die einzelnen Vertreter der Mitgliedstaaten aus ihrer doppelten Mitgliedschaft sowohl in den nationalen Zentralbanken als auch im Ausschuß Konflikte ergeben.

Im zweiten Beschluß wird die multilaterale Überwachung als Instrument der Koordinierung dargestellt, die frühere Koordinierungsvorschriften ${ }^{63}$ ablöst. Der Rat prüft dabei mindestens zweimal jährlich die wirtschaftliche Lage, die

59 Vgl. Rat der EG =Zusammenarbeit zwischen den Zentralbanken $1990=25 \mathrm{f}$.

$60 \mathrm{Vgl}$. Rat der EG =Schrittweise Konvergenz 1990= 23f.

$61 \mathrm{Vgl}$. Rat der EG =Zusammenarbeit zwischen den Zentralbanken $1990=25 \mathrm{f}$.

62 Vgl. im folgenden Herz, Nürk =Erste Phase $=318$.

63 Vgl. vor allem Rat der EG =Konvergenz 1974=16ff. 


\section{Aufgabenverteilung}

Aussichten und die Wirtschaftspolitik in der Gemeinschaft und ihren Mitgliedstaaten, die Vereinbarkeit der Wirtschaftspolitik in den Mitgliedstaaten und in der Gemeinschaft insgesamt sowie das außenwirtschaftliche Umfeld und seine Wechselwirkungen mit der Wirtschaft der Gemeinschaft. Zur Überwachung zieht der Rat insbesondere regelmäßige Länderberichte und den Jahreswirtschaftsbericht der Gemeinschaft heran. ${ }^{64}$

Der Rat kann wirtschaftspolitische Anregungen geben oder wirtschaftspolitische Empfehlungen aussprechen. ${ }^{65}$ Bezüglich der Haushaltspolitik der einzelnen Mitgliedstaaten sollte er die nationalen Haushaltspläne möglichst vor deren Feststellung überprüfen. Allerdings bleibt diese Regel unverbindlich, da keine Sanktionen vorgesehen sind, die eine Abstimmung der Haushaltspläne erzwingen könnten. Den Mitgliedstaaten wird letztlich nur ein Diskussionsforum zur Verfügung gestellt, innerhalb dessen die internationalen Effekte nationaler Maßnahmen besprochen werden können. ${ }^{66}$ Insgesamt gehen von den bisher getroffenen Koordinierungsregeln keine größeren Impulse hinsichtlich einer verbindlicheren Abstimmung der Fiskalpolitik aus. ${ }^{67}$

Ende Oktober 1990 beschloß der Europäische Rat den Beginn der zweiten Phase der Wirtschafts- und Währungsunion für den 1.1.1994, sofern bestimmte Voraussetzungen erfüllt sind. In ihr soll eine "neue Institution" gegründet werden. ${ }^{68}$ Die Rolle dieser Institution ist nicht ganz klar, da die geldpolitische Souveränität auch in der zweiten Phase grundsätzlich bei den nationalen Zentralbanken bleiben soll. ${ }^{69}$ Erst mit dem Beginn der dritten Phase soll die geldpolitische Verantwortung in vollem Umfang auf die Europäische Zentralbank übergehen.

\section{e) Wirtschaftlicher und sozialer Zusammenhalt}

Der ursprüngliche Vertrag zur Gründung der Europäischen Wirtschaftsgemeinschaft erteilte den Organen keinen generellen Auftrag in bezug auf regio-

64 Vgl. Rat der EG =Schrittweise Konvergenz 1990 $=24$.

65 Vgl. Rat der EG =Schrittweise Konvergenz 1990= 24.

66 Vgl. Herz, Nürk $=$ Erste Phase $=316$ und Hirn $=$ Großes Vorbild Fed $=32$.

67 So auch Herz, Nürk =Erste Phase $=317$ und Hirn $=$ Großes Vorbild Fed $=32$.

68 Vgl. Europäischer Rat $=$ Schlußfolgerungen $1990=1334 \mathrm{f}$.

69 Vgl. dazu: Sachverständigenrat zur Begutachtung der gesamtwirtschaftlichen Entwicklung = Jahresgutachten 1990/91 = Ziff. 486. 
nalpolitische Maßnahmen. Die Gründungsmitglieder der Gemeinschaft gingen davon aus, daß die Marktintegration der geeignete Weg sei, um das erklärte Ziel der Verringerung der interregionalen Disparitäten zu erreichen.

Diese Einstellung änderte sich im Laufe der sechziger Jahre grundlegend, da man festzustellen meinte, daß die Marktintegration den Verdichtungsräumen tendenziell stärker zugute komme als den peripheren Regionen. Es wurde in zunehmendem Maße eine aktive Regionalpolitik gefordert, die schließlich am 18. März 1975 in Form des Euopäischen Fonds für regionale Entwicklung verwirklicht wurde. ${ }^{70}$ Durch die Einheitliche Europäische Akte wurde 1987 die Regionalpolitik auch vertraglich verankert.

Nach Art. 130a Satz 2 EWGV verfolgt die Gemeinschaft das Ziel, den Abstand zwischen den verschiedenen Regionen und den Rückstand der am wenigsten begünstigten Gebiete zu verringern. Die Mitgliedstaaten sollen ihre Wirtschaftspolitik in Abstimmung mit diesem Ziel führen und koordinieren. Auch bei der Durchführung der gemeinsamen Politik der Gemeinschaft und bei der Errichtung des Binnenmarktes ist dieses Ziel zu beachten. Die Gemeinschaft unterstützt diese Bemühungen durch ihre Politik im Rahmen der Strukturfonds (Europäischer Ausrichtungs- und Garantiefonds für die Landwirtschaft - Abteilung Ausrichtung, Europäischer Sozialfonds und Europäischer Fonds für regionale Entwicklung) sowie durch die Europäische Investitionsbank. ${ }^{71}$

Durch Art. 130c EWGV wurde der schon seit 1975 bestehende Europäische Fonds für regionale Entwicklung im Vertrag verankert und seine Aufgaben wie folgt definiert: Ausgleich der wichtigsten regionalen Ungleichgewichte durch Beteiligung an der Entwicklung der rückständigen Gebiete und Umstellung der Industriegebiete mit rückläufiger Entwicklung.

In Art. 130d EWGV wurde schließlich eine Reform der Strukturfonds angekündigt, die die Effizienz der Fonds erhöhen und ihre Tätigkeit sowohl untereinander als auch im Verhältnis zu anderen Finanzierungsinstrumenten koordinieren sollte. Diesem Auftrag wurde durch die Verordnung "Über Aufgaben und Effizienz der Strukturfonds und über die Koordinierung ihrer Interventionen untereinander sowie mit denen der Europäischen Entwick-

$70 \mathrm{Vgl}$. zu dieser Entwicklung Franke = Regionalpolitik der EG=10f.

71 Vgl. Art. 130 b EWGV. 
lungsbank und der anderen vorhandenen Finanzierungsinstrumente"72 und den entsprechenden Durchfuihrungsverordnungen ${ }^{73}$ Rechnung getragen.

Aus den allgemeinen Zielvorgaben der Art. 130a und 130c EWGV wurden fünf vorrangige Ziele abgeleitet, zu deren Verwirklichung die drei Fonds beitragen sollen.

Tabelle 1: Die Ziele der Strukturfonds

Ziele

beteiligte Fonds

1. Förderung der Entwicklung und der strukturellen Anpassung der Regionen mit Entwicklungsrückstand

EAGFL-Ausrichtung EFRE, ESF

2. Umstellung der Regionen, Grenzregionen und TeilreEFRE, ESF gionen (einschließlich Arbeitsmarktregionen und städtischer Verdichtungsräume), die von der rückläufigen industriellen Entwicklung stark betroffen sind

3. Bekämpfung der Langzeitarbeitslosigkeit ESF

4. Erleichterung der Eingliederung der Jugendlichen ESF in das Erwerbsleben

5. Im Hinblick auf die Reform der Gemeinsamen Agrarpolitik:

a) beschleunigte Anpassung der Agrarstrukturen EAGFL-Ausrichtung

b) Förderung der Entwicklung des ländEAGFL-Ausrichtung lichen Raums EFRE, ESF

EFRE = Europäischer Fonds für regionale Entwicklung

ESF = Europäischer Sozialfonds

EAGFL = Europäischer Ausgleichs- und Garantiefonds für die Landwirtschaft

72 Rat der EG = Aufgaben und Effizienz der Strukturfonds $1988=9 \mathrm{ff}$.

73 Vgl. Rat der EG =Koordinierung der Strukturfonds 1988=1ff. 
Die Ziele sollen in enger Zusammenarbeit zwischen der Kommission und dem entsprechenden Mitgliedstaat verfolgt werden. Die Strukturpolitik läßt sich damit eindeutig zu den konkurrierenden Aufgaben der Gemeinschaft zählen. Zunächst stellen nationale Behörden regionale Pläne (für die Ziele Nr. 1, 2 und 5b)) und nationale Pläne (für die Ziele Nr. 3 und 4) auf. ${ }^{74}$ Die eingereichten Pläne sind auf derjenigen nationalen Ebene auszuarbeiten, die für am geeignetsten gehalten wird. "Sie werden von den zuständigen Stellen, die der Mitgliedstaat auf nationaler, regionaler oder sonstiger Ebene benennt, erstellt und von dem Mitgliedstaat der Kommission vorgelegt." 75 Diese Pläne sollen mit den Plänen abgestimmt werden, die sich innerhalb eines Mitgliedstaates auf das gleiche Ziel beziehen, und darüber hinaus auch mit Projekten koordiniert werden, die sich im Rahmen verschiedener Fonds auf das gleiche Ziel beziehen. In der Bundesrepublik Deutschland werden die Pläne von den Landesbehörden ausgearbeitet. ${ }^{76}$ Sie enthalten eine sozioökonomische Analyse der Region, des Sektors oder der betreffenden Probleme, die Entwicklungsstrategie, die gewählten Verfahren, die eingesetzten Mittel, nationale und regionale Finanzierungen, bereits laufende Gemeinschaftsmaßnahmen, zu finanzierende Förderschwerpunkte und die beantragten Gemeinschaftsmittel, aufgeschlüsselt nach den Strukturinstrumenten. ${ }^{77}$

Buchstabe j des Art. 3 EWGV sieht schließlich die Schaffung einer Europäischen Investitionsbank vor. Diese soll zu einer ausgewogenen und reibungslosen Entwicklung des Gemeinsamen Marktes beitragen. Sie besitzt eine eigene Rechtspersönlichkeit und erleichtert durch Gewährung von Darlehen und Bürgschaften Vorhaben zur Erschließung der weniger entwickelten Gebiete, Vorhaben zur Modernisierung oder Umstellung von Unternehmen oder zur Schaffung neuer Arbeitsmöglichkeiten und Vorhaben von gemeinsamem Inter-

74 Maßnahmen für das Ziel 5a) werden von der Gemeinschaft geplant.

75 Rat der EG =Koordinierung der Strukturfonds $1988=4$.

76 Vgl. Deutscher Städtetag $=$ Binnenmarkt und Städte $=22$.

77 Da eine Festlegung der Regionen und Gebiete nach den einzelnen Zielen wegen der fehlenden Datenbasis für die neuen Länder nicht möglich war, wurde für sie ein gemeinschaftliches Förderkonzept mit erleichterten Anspruchsvoraussetzungen entwikkelt. Vgl. dazu Presse- und Informationsamt der Bundesregierung =Einbeziehung= 14. 
esse für mehrere Mitgliedstaaten. ${ }^{\mathbf{7 8}}$ Die Vorhaben werden insbesondere dann von der Europäischen Investitionsbank unterstützt, wenn sie wegen ihres Umfangs oder ihrer Art mit den in den einzelnen Mitgliedstaaten vorhandenen Mitteln nicht vollständig finanziert werden können. Zur Kreditvergabe greift die Europäische Investitionsbank sowohl auf eigene Mittel der Bank (eingezahlte Mittel und Mittel aus Anleihen, die die Bank begeben hat) als auch auf Mittel aus dem sogenannten Neuen Gemeinschaftsinstrument zurück. Bei letzterem gibt die Europäische Investitionsbank Darlehen aus Mitteln, die die Kommission im Namen der Europäischen Wirtschaftsgemeinschaft auf den Kapitalmärkten aufgenommen hat. ${ }^{79} \mathrm{Da}$ die Europäische Investitionsbank keinen Erwerbszweck verfolgt, berechnet sie für Kredite, zu deren Finanzierung Darlehen auf dem Kapitalmarkt aufgenommen wurden, nur die Beschaffungskosten, so daß der Kreditnehmer in den Genuß der erstklassigen Kreditwürdigkeit der Europäischen Investitionsbank kommt. ${ }^{80}$

\section{f) Forschung und technologische Entwicklung}

Durch die Einheitliche Europäische Akte wurde auch der Titel VI "Forschung und technologische Entwicklung" dem Vertrag zur Gründung der Europäischen Wirtschaftsgemeinschaft neu hinzugefügt (Art. 130f bis 130q EWGV). Bis 1987 bestanden nur sektorale Forschungszuständigkeiten, z. B. im Agrarsektor (Art. 41 EWGV), auf dem Gebiet der Kernforschung (Art. 4ff. EAGV) und im Rahmen des Vertrages zur Gründung der Europäischen Gemeinschaft für Kohle und Stahl auf dem Gebiet der Stahl- und Kohleforschung (Art. 55 EGKSV).

Die Forschungs- und Technologiepolitik der Gemeinschaft hat die Aufgabe, die wissenschaftlichen und technischen Grundlagen der europäischen Industrie zu stärken (Art. 130f EWGV). Um dies zu erreichen, soll die Gemeinschaft folgende Maßnahmen treffen, die die in den Mitgliedstaaten durchgeführten Aktionen ergänzen: ${ }^{81}$

78 Neben regionalpolitisch motivierten Finanzierungen gewährt die Europäische Investitionsbank auch Kredite für andere Ziele, wie z. B. die Versorgungssicherheit bei Energie, den Umweltschutz oder die Stärkung der Wettbewerbsfähigkeit der klein- und mittelständischen Industrie.

79 Vgl. Rat der EG = Neues Gemeinschaftsinstrument $=34 \mathrm{ff}$. und Abschnitt C. III. 1.

80 Vgl. dazu Schäfers, Starbatty $=$ Kohäsion $=6$.

81 Vgl. Art. 130g EWGV. 
a) Durchführung von Programmen für Forschung, technologische Entwicklung und Demonstration unter Förderung der Zusammenarbeit mit Unternehmen, Forschungszentren und Hochschulen;

b) Förderung der Zusammenarbeit mit Drittländern und internationalen Organisationen auf dem Gebiet der gemeinschaftlichen Forschung, technologischen Entwicklung und Demonstration;

c) Verbreitung und Auswertung der Ergebnisse dieser Tätigkeit;

d) Förderung der Ausbildung und der Mobilität der Forscher aus der Gemeinschaft.

Eine Konkretisierung erfährt die gemeinschaftliche Forschungs- und Technologiepolitik durch die Aufstellung von mehrjährigen Rahmenprogrammen. In diesen werden die wissenschaftlichen und technischen Ziele, ihre jeweilige Prioritätsstufe und die Grundzüge der geplanten Aktionen festgelegt. Außerdem werden der für notwendig erachtete Betrag und die Einzelheiten der finanziellen Beteiligung der Gemeinschaft hinsichtlich Höhe und Aufteilung auf die verschiedenen geplanten Aktionen festgeschrieben. ${ }^{82}$ Zur Umsetzung des Rahmenprogramms werden spezifische Programme aufgestellt. ${ }^{83}$ Dabei gibt es verschiedene Gestaltungsmöglichkeiten: ${ }^{84}$

1. Bei den direkten Aktionen betreibt die Gemeinschaft Eigenforschung in der Gemeinsamen Forschungsstelle. Diese wurde zunächst auf der Basis des Vertrags zur Gründung der Europäischen Atomgemeinschaft eingerichtet, ihre Forschungsschwerpunkte erweiterten sich jedoch im Laufe der Zeit. Die Gemeinsame Forschungsstelle hat öffentlich-rechtlichen Charakter und wird bisher voll aus Gemeinschaftsmitteln finanziert.

2. Indirekte Aktionen beinhalten Programme mit Forschungsverträgen auf der Basis der Kostenteilung. Unternehmen und Forschungsinstitute können transnationale Projekte vorschlagen, die zum vorgegebenen Rahmenprogramm passen. Die genehmigten Projekte werden in der Regel mit $50 \%$ der entstehenden Kosten gefördert. Diese Förderungsform nimmt den größten Umfang innerhalb der Forschungsförderung ein.

82 Vgl. Art. 130i EWGV.

83 Vgl. Art. 130k EWGV.

84 Vgl. zu den folgenden Ausführungen Starbatty, Vetterlein $=$ Technologiepolitik= $67 \mathrm{ff}$. 
3. Bei konzertierten Aktionen versucht die Gemeinschaft supranationale Forschungsaktivitäten anzustoßen und zu koordinieren. Dabei leistet die Gemeinschaft keinen Beitrag zur Finanzierung der Projekte, "sondern organisiert - allerdings auf eigene Kosten - die Zusammenarbeit in der wissenschaftlichen und technischen Forschung über die nationalstaatlichen Grenzen hinweg"85. Kooperationspartner sind in der Regel öffentliche Forschungseinrichtungen; die Verträge werden auf Regierungsebene geschlossen. Bei der angestrebten weitergehenden Abstimmung nationaler und gemeinschaftlicher Technologiepolitik kamen die Ansätze bisher noch nicht über eine empirische Erfassung der techologiepolitischen Aktivitäten in den einzelnen Mitgliedstaaten und die Verabschiedung wohlklingender Absichtserklärungen hinaus.

4. Schießlich gibt es noch horizontale Aktionen, die einen Beitrag zur $\mathrm{Zu}$ kunftsforschung, zur Bewertung und Stimulierung von Forschungsaktivitäten allgemein und zum Wissenschaftsaustausch liefern sollen. Diese Aktionen werden hauptsächlich als "Vertragsforschung" organisiert, wobei sich die Gemeinschaft mit bestimmten Sätzen an den einzelnen Projekten beteiligt.

Den nationalen Ebenen verbleiben noch wichtige Aufgabenfelder im Rahmen der Technologiepolitik; so finanziert in der Bundesrepublik Deutschland der Bund weiterhin Projekte der Großforschung, schafft den Ordnungsrahmen (etwa das Patentrecht) und kann steuerliche Anreize zur Innovationsförderung setzen. Die Länder liefern einen Beitrag zur Technologiepolitik im Rahmen ihrer Bildungs- und Hochschulpolitik. Oft trägt die von den Ländern betriebene Mittelstandsförderung ebenfalls technologiepolitische Züge. ${ }^{86}$ Von Seiten der Europäischen Gemeinschaft besteht allerdings die Tendenz, nationale Forschungsprogramme mit Hilfe ihrer Beihilfeaufsichtskompetenz nach Art. 93 EWGV zu kontrollieren. Die Problematik der Beihilfeaufsicht, die sich nicht nur bei der Forschungsförderung stellt, wird später genauer diskutiert. Die Gemeinschaft betont zusehends stärker ihre Zuständigkeit bei Fragen der Normung und dem Setzen von Standards. ${ }^{87}$ Die Aufgabenverlagerung hin zur Gemeinschaftsebene bei konkreten Forschungsvorhaben vollzieht sich außerdem

85 Starbatty, Vetterlein $=$ Technologiepolitik $=71$.

86 Vgl. dazu auch Starbatty, Vetterlein $=$ Technologiepolitische Aufgabenteilung $=96$.

87 Vgl. Starbatty, Vetterlein $=$ Technologiepolitik $=73$. 
häufig nach dem folgenden Muster: "Die Gemeinschaft beweist ... mit zunächst kleinen Programmen ihre fachliche Zuständigkeit; diese kleinen Programme werden später ausgedehnt und so miteinander verknüpft, daß ein komplettes Netzwerk an technologiepolitischen Aktivitäten entsteht, die den nationalen Programmen den Rang ablaufen." 88

\section{g) Umweltpolitik}

Schließlich wurde durch die Einheitliche Europäische Akte auch die gemeinschaftliche Umweltpolitik in den Verträgen verankert. Diese verfolgt das Ziel, die Umwelt zu erhalten, zu schützen und ihre Qualität zu verbessern, zum Schutz der menschlichen Gesundheit beizutragen sowie eine umsichtige und rationelle Verwendung der natürlichen Ressourcen zu gewährleisten (Art. 130r Abs. 1 EWGV). Die Gemeinschaft soll im Bereich der Umwelt nur insoweit tätig werden, als die gemeinsamen umweltpolitischen Ziele besser auf Gemeinschaftsebene erreicht werden können als auf der Ebene der Mitgliedstaaten (Art. 130r Abs. 4 Satz 1 EWGV).

Die Gemeinschaft hat zahlreiche umweltpolitische Richtlinien erlassen. ${ }^{89}$ Schwerpunkte der gemeinschaftlichen Umweltpolitik bilden der Gewässerschutz (z. B. mit Richtlinien über die Einleitung gefährlicher Stoffe in die Gewässer der Gemeinschaft ${ }^{\mathbf{9 0}}$, über den Schutz des Grundwassers ${ }^{91}$ und über die Qualität von Wasser für den menschlichen Verbrauch ${ }^{92}$ ) und die Luftreinhaltung (z. B. mit der Richtlinie über Grenzwerte und Leitwerte der Luftqualität für Schwefeldioxid und Schwebstaub ${ }^{\mathbf{9 3}}$ oder der Richtlinie zur Begrenzung von Schadstoffemissionen von Großfeuerungsanlagen ${ }^{94}$ ). Im Mittelpunkt der gemeinschaftlichen Umweltforschung steht die Erforschung der Auswirkungen von Schadstoffen auf die Gesundheit und die Risiken im Zusammenhang mit Chemikalien und bei der Klimaforschung. ${ }^{95}$

88 Starbatty, Vetterlein $=$ Technologiepolitik $=118$.

89 Einen Überblick bietet Rat der EG =Aktionsprogramm Umweltschutz 1987-1992=.

90 Vgl. Rat der EG = Ableitung gefährlicher Stoffe 1976= 23ff.

91 Vgl. Rat der EG $=$ Schutz des Grundwassers $1979=43 \mathrm{f}$.

92 Vgl. Rat der EG =Wasserqualităt $1980=11 \mathrm{ff}$.

93 Vgl. Rat der EG $=$ Grenzwerte der Luftqualität $1980=30$ f.

94 Vgl. Rat der EG =Schadstoffemissionen von Großfeuerungsanlagen 1988=1ff. 
In der Regel sind die Mitgliedstaaten für die Finanzierung und die Durchführung der umweltpolitischen Gemeinschaftsmaßnahmen verantwortlich (Art. 130r Abs. 4 Satz 2). Sehen die umweltpolitischen Richtlinien der Europäischen Gemeinschaften konkrete Normen vor, so bleibt den Mitgliedstaaten kein Spielraum; sie sind an diese Vorgaben gebunden. Die Umsetzung von Umweltrichtlinien in nationales Recht erfolgt allerdings häufig erst mit Verzögerung und nach Mahnungen durch die Kommission.96

Für die nationale Umweltpolitik sind aber nicht nur die Umweltrichtlinien der Europäischen Gemeinschaften bedeutsam, sondern auch die wettbewerbspolitischen Vorgaben der Europäischen Gemeinschaften. Das Postulat der einheitlichen Wettbewerbsbedingungen verhindert vielfach die Durchsetzung strengerer nationaler Normen. Probleme gibt es vor allem bei produktbezogenen Umweltvorschriften, die häufig als nicht-tarifäre Handelshemmnissse betrachtet werden. 97

\section{Die Aufgaben der Gemeinschaften und die nationalen Ebenen Bund, Länder und Gemeinden}

Die bisherigen Ausführungen haben deutlich gemacht, daß die Europäischen Gemeinschaften die Zahl ihrer Aufgabenfelder im Laufe der Zeit stark erhöht hat: Erstens sind Aufgaben, die die Europäischen Gemeinschaften de facto ohne rechtliche Grundlage wahrgenommen haben, schließlich in den Verträgen verankert worden, so z. B. die Umweltpolitik und die Regionalpolitik durch die Einheitliche Europäische Akte. Zweitens beeinflussen die Europäischen Gemeinschaften durch die Harmonisierungsvorschriften im Rahmen der Vollendung des Binnenmarktes zunehmend nationale Aufgaben, für die sie auch heute noch keine vertraglichen Zuständigkeiten besitzen, wie z. B. bei der Fernsehrichtlinie. Drittens ist festzustellen, daß auch die Intensität der gemeinschaftlichen Wahrnehmung von Aufgaben bei Politikbereichen steigt, in denen sowohl die Europäischen Gemeinschaften als auch die Mitgliedstaaten Kompetenzen besitzen, wofür insbesondere die Technologiepolitik ein gutes Beispiel bietet.

95 Vgl. Rat der EG =STEP und EPOCH 1989=9ff.

96 Vgl. dazu Kommission der EG $=$ XXIII. Gesamtbericht $=239$. Ein aktueller Fall in der Bundesrepublik Deutschland ist die Umsetzung der Richtlinie über die Qualität von Trinkwasser, vgl. dazu o. V. =Trinkwasserverordnung $=4$.

97 Vgl. dazu genauer Abschnitt D. I. 3. a). 
Sofern die Zunahme der Aufgaben der Europäischen Gemeinschaften nicht zu einer Ausweitung des Bestandes an Aufgaben des öffentlichen Sektors insgesamt führt, muß dieser Funktionszuwachs auf Kosten der Aufgaben darunter liegender föderativer Ebenen gehen. ${ }^{98}$ In der Literatur finden sich zu dieser Frage zwei unterschiedliche Haltungen. Auf der einen Seite wird die Meinung geäußert, daß vor allem der Bund - bedrängt von den stärker werdenden Europäischen Gemeinschaften und den Ländern - derjenige sei, von dem Kompetenzen nach Brüssel abwandern. ${ }^{99}$ Zimmermann spricht hier von der "SandwichThese" ${ }^{100}$. Auf der anderen Seite wird die Ansicht vertreten, daß der Bund seine Machtposition weitgehend behalte, während die Länder an Bedeutung verlören. ${ }^{101}$ Diese Auffassung würde "zu einer Art generalisierter Popitzscher These passen, daß auf Dauer eine Tendenz zur Zentralisierung im Gefüge der Ebenen besteht"102. Welche dieser beiden Auffassungen die größere Plausibilität für sich beanspruchen kann, soll im folgenden geprüft werden.

\section{a) Die Auswirkungen von Regelungen der Europäischen Gemeinschaften auf Bund, Länder und Gemeinden}

\section{aa) Auswirkungen auf den Bund}

Die Verlagerung von Aufgaben auf die Gemeinschaften betrifft zunächst vor allem die Ebene des Bundes. Die ausschließliche Gesetzgebungskompetenz des Bundes wird insbesondere in den Bereichen der Zoll- und Handelspolitik, der Währungspolitik, der Freizügigkeit und der Verkehrspolitik berührt. Die Weiterentwicklung der Gemeinschaften hin zur Wirtschafts- und Währungsunion wird für den Bund weitere Kompetenzverluste bei der Wirtschaftspolitik mit sich bringen. Die konkurrierende Gesetzgebung des Bundes wird durch Regelungen der Europäischen Gemeinschaften zur Niederlassungsfreiheit, zum Bergbau, zur Energiewirtschaft, zum Arbeitsrecht sowie zur Förderung der landwirtschaftlichen und forstwirtschaftlichen Erzeugung beschnitten.

98 Vgl. Zimmermann $=$ Gewichtsverlagerungen $=453$.

99 So z. B. Wessels = Deutsche Länder in der EG-Politik= $181 \mathrm{ff}$, , insbesondere 182 und Hellwig $=$ Rolle der Bundesländer $=297 \mathrm{ff}$.

100 Zimmermann $=$ Gewichtsverlagerungen $=453$.

101 So vor allem Hrbek $=$ Politikverflechtung $=38 \mathrm{ff}$.

102 Zimmermann $=$ Gewichtsverlagerungen $=454$. 
Es liegt in der Natur der Sache, daß in erster Linie zentralstaatliche Funktionen auf die Gemeinschaften übertragen werden. Doch auch die Länder und die Gemeinden werden mit Gemeinschaftsregelungen konfrontiert, die ihre Aufgaben betreffen und die den politischen Gestaltungsspielraum dieser Ebenen einschränken.

\section{bb) Auswirkungen auf die Länder}

Durch die Maßnahmen zur Vollendung des Binnenmarktes sehen sich die Länder Maßnahmen zur Angleichung der Rechts- und Verwaltungsvorschriften gegenüber, die in die ihnen nach dem Grundgesetz zugeordneten Aufgabenbereiche hineinwirken. Dies gilt besonders für die Bereiche der Medienpolitik $^{103}$, der Bildungspolitik ${ }^{104}$, der Umweltpolitik ${ }^{105}$, des Verbraucherschutzes und der Gesundheitspolitik ${ }^{106}$.

Auch bei der Regionalpolitik, die die Länder vor allem im Rahmen der Gemeinschaftsaufgabe "Verbesserung der regionalen Wirtschaftsstruktur" gemeinsam mit dem Bund, aber auch durch eigene Fördermaßnahmen wahrnehmen, wird der Einfluß der Europäischen Gemeinschaften deutlich. Diese sind einerseits bei der Durchführung der gemeinschaftlichen Regionalpolitik auf die Mitarbeit des Bundes und der Länder angewiesen, andererseits üben sie durch ihre Beihilfenkontrolle nach Art. 92 und 93 EWGV direkten Einfluß auf die deutsche Regionalpolitik aus. Gemäß Art. 93 Abs. 2 EWGV überprüft die Kommission, ob die Beihilfen der Mitgliedstaaten mit dem Gemeinsamen Markt nach Art. 92 EWGV unvereinbar sind oder mißbräuchlich angewandt werden. So muß beispielsweise der Rahmenplan der Gemeinschaftsaufgabe jährlich der Kommission notifiziert und von ihr gebilligt werden. ${ }^{107}$ Die Kommission kann von den Mitgliedstaaten ausgewiesene Fördergebiete für

103 Vgl. z. B. Rat der EG $=$ Fernseh-Richtlinie $1989=23 \mathrm{ff}$.

104 Vgl. z. B. Rat der EG $=$ Kinder von Wanderarbeitnehmern $1976=32 \mathrm{ff}$. und Rat der $\mathrm{EG}=$ Anerkennung von Hochschuldiplomen $1988=16 \mathrm{ff}$.

105 Da die Länder insbesondere für den Gewässerschutz zuständig sind, sei hier als Beispiel die Richtlinie über die Qualitätsanforderungen an Oberflächenwasser für die Trinkwassergewinnung genannt, vgl. Rat der EG =Qualitätsanforderungen $1975=$ $34 \mathrm{ff}$.

106 Z. B. durch die Richtlinie über Zusatzstoffe für Lebensmittel und die diesbezügliche Kennzeichnung sowie über kosmetische, pharmazeutische und sonstige chemische Produkte, vgl. dazu Beutler u. a. =Europäische Gemeinschaft $=502$.

$107 \mathrm{Vgl}$. Bundesministerium für Wirtschaft $=$ Regionalpolitik $=33$. 
förderungsunwürdig erklären und veranlassen, daß entsprechende Gebiete aus der Förderung herausgenommen werden. So mußten etwa nach der Fördergebietsabgrenzung der Gemeinschaftsaufgabe 1981 neun der als förderbedürftig angesehenen Regionen aufgrund der Verbotsentscheidung der Kommission aus der Förderung herausgenommen werden. ${ }^{108}$ Auch gegen Förderprogramme einzelner Länder hat die Kommission nach Art. 93 EWGV Wettbewerbsverfahren eingeleitet, z. B. gegen die Beihilfen des Landes Baden-Württemberg im Jahre $1984^{109}$, gegen die Vergabe von Regionalbeihilfen des Landes Nordrhein-Westfalen im Jahre $1985^{\mathbf{1 1 0}}$ und gegen das Beihilfeprogramm zur Abwanderungsverminderung der Freien und Hansestadt Hamburg im Jahre 1991111. Die Tatsache, daß die Förderung des Landes Baden-Württemberg für die Ansiedlung eines Werkes der Firma Daimler Benz im strukturschwachen Raum Rastatt auf Entscheidung der Kommission zurückgenommen werden mußte, stellt ein weiteres aktuelles Beispiel für die Anwendung der Beihilfeaufsicht der Europäischen Gemeinschaften dar. ${ }^{112}$ Ähnliche Beispiele lassen sich im übrigen auch für die Agrarstrukturpolitik anführen. ${ }^{113}$

\section{cc) Auswirkungen auf die Gemeinden}

Die Gemeinschaftsregelungen wirken auf einigen Gebieten bis in den kommunalen Bereich. Die gemeinschaftliche Beihilfenkontrolle hat für die kommunale Wirtschaftsförderung ähnliche Konsequenzen wie für die Regionalpolitik auf Landesebene, denn sie richtet sich unmittelbar gegen alle von den Gemeinden an Unternehmen gewährten Leistungen. "Dies betrifft nicht nur Geldleistungen, sondern auch sonstige geldwerte Vorteile, etwa durch Verzicht auf

$108 \mathrm{Vgl}$. Planungsausschuß für regionale Wirtschaftsstruktur $=16$. Rahmenplan $=24$.

109 Als nicht vereinbar mit dem Gemeinsamen Markt wurden u. a. die Beihilfen nach den Richtlinien des Ministeriums für Wirtschaft, Mittelstand und Verkehr des Landes Baden-Württemberg für die Gewährung von Zuwendungen in den Fördergebieten des Landes erklärt. Vgl. Kommission der EG = Regionale Beihilfen $1984=3$.

110 Die Kommission sprach sich gegen die Vergabe von Regionalbeihilfen in den Arbeitsmarktregionen Borken-Bocholt, Siegen und Gummersbach aus. Vgl. Kommission der EG $=$ Wirtschaftsförderungsprogramm Nordrhein-Westfalen $1985=28 \mathrm{ff}$.

111 Vgl. Kommission der EG = Beihilfen der Freien und Hansestadt Hamburg $=1 \mathrm{ff}$.

112 Vgl. Kommission der EG = Bulletin 1987= 47. Zu der Position der Landesregierung vgl. Rengeling $=$ Subventionsrecht $=459$. Zu den Differenzen zwischen der Bundesrepublik Deutschland und der Gemeinschaft in der Frage der Beihilfen zugunsten der Automobilindustrie allgemein, vgl. Kommission der EG = Beihilferegelungen zugunsten der Kfz-Industrie $1990=55 \mathrm{ff}$.

113 Vgl. Rösslein $=$ Landesparlamente $=82$. 
Gebühren und Beiträge, Verbilligung von Grundstückspreisen, Übernahme von Garantien und Bürgschaften sowie von sonstigem Aufwand, den ein wirtschaftliches Unternehmen im normalen Wettbewerb selbst zu tragen hätte. Damit sind weite Teile der traditionellen kommunalen Wirtschaftsförderung berührt, aber auch die Erfüllung kommunaler Aufgaben mit Unternehmen in privater Rechtsform."114 Durch Gemeinschaftsregelungen tangiert werden auch das Bauwesen und das Bauvergabewesen ${ }^{115}$ sowie die Vergabe anderer öffentlicher Aufträge ${ }^{116}$. Schließlich könnte in der Zukunft der Vorschlag für eine Richtlinie über das Wahlrecht der Staatsangehörigen der Mitgliedstaaten bei den Kommunalwahlen im Aufenthaltsstaat für die Gemeinden bedeutsam werden. ${ }^{117}$

Insgesamt wird deutlich, daß die vierte Ebene auf alle Ebenen des föderativen Aufbaus der Bundesrepublik Deutschland einwirkt. Allerdings sei noch einmal betont, daß sich die meisten Auswirkungen auf der Ebene des Bundes zeigen. Diese Tatsache scheint zunächst die "Sandwich-These" zu stützen. Andererseits ist jedoch zu beachten, daß der Bund im Gegensatz zu Ländern und Gemeinden über eine viel größere Zahl von Aufgaben verfügt. Er verkraftet insofern gewisse Kompetenzeinbußen leichter. Die Länder sehen sich dagegen schnell in ihrem Kern angegriffen. ${ }^{118}$ Außerdem besitzt der Bund auch die größten Mitwirkungsmöglichkeiten beim Prozeß der gemeinschaftlichen Rechtsetzung. Auf diesen Aspekt soll im folgenden Abschnitt näher eingegangen werden.

\section{b) Der Einfluß von Bund und Ländern auf die gemeinschaftliche Recht- setzung}

Durch Art. 24 Abs. 1 GG ist der Bund berechtigt, Hoheitsrechte auf zwischenstaatliche Einrichtungen zu übertragen. Nach herrschender Meinung ermäch-

114 Deutscher Städtetag = Binnenmarkt und Städte $=24$.

115 Seit 1988 ist die Bauproduktrichtlinie in Kraft, für den Bereich der Bauleistungen ist die novellierte Baukoordinierungsrichtlinie im Sommer 1988 verabschiedet worden, vgl. Deutscher Städtetag $=$ Binnenmarkt und Städte $=29 \mathrm{f}$. Vgl. auch Rat der EG $=$ Vergabe öffentlicher Bauaufträge 1989=1ff.

116 Vgl. dazu Deutscher Städtetag $=$ Binnenmarkt und Städte $=30 \mathrm{ff}$. und $0 . \mathrm{V}$. $=$ Offentliche Aufträge $=2$.

117 Vgl. Kommission der EG = Vorschlag Kommunalwahlrecht $=4 \mathrm{ff}$.

118 Vgl. Graf Vitzthum =Föderalismus $=291$. 
tigt diese Bestimmung den Bund, nicht nur eigene, sondern auch Kompetenzen der Länder auf die Europäischen Gemeinschaften zu übertragen, und zwar durch einfaches Gesetz. 119

Die Verlagerung von Aufgaben sowohl des Bundes als auch der Länder auf die Europäischen Gemeinschaften hat Auswirkungen auf das Verhältnis zwischen Bund und Ländern. Werden Gesetzgebungskompetenzen des Bundes auf die Gemeinschaften übertragen, so verliert zwar auf den ersten Blick vor allem der Bund Kompetenzen, doch sind auch die Länder indirekt davon betroffen, da sie bisher durch den Bundesrat auf entsprechende Gesetzesbeschlüsse Einfluß nehmen konnten. Zwar könnte man den Verlust des Mitwirkungsrechtes des Bundesrates im Hinblick darauf akzeptieren, daß jetzt auch andere Bundesorgane - z. B. der Bundestag - von der Regelung der Materie ausgeschlossen sind, doch ist die Bundesregierung aufgrund ihrer Mitwirkungsrechte nach Gemeinschaftsrecht - vor allem durch ihren Vertreter im Ministerrat - tatsächlich von der Beteiligung bei der Regelung dieser Materie nicht vollständig ausgeschlossen ${ }^{120}$. Allerdings bedeutet das Stimmrecht im Rat eine bescheidenere Machtposition als eine eigene Regelungskompetenz, zumal bei Mehrheitsbeschlüssen die Gefahr besteht, überstimmt zu werden. Dennoch kann die Bundesregierung den Verlust alter Kompetenzen teilweise durch neue Kompetenzen im Rat ausgleichen, während bei Bundestag und Bundesrat ein sehr starker Kompetenzverlust zu verzeichnen ist: "Die 'Europäisierung' einer dem Bund zur Regelung zustehenden Materie hat nicht nur einen Verlust parlamentarisch-demokratischer Substanz, sondern auch an Bundesstaatlichkeit zur Folge."121

Auch durch die Verlagerung von Länderaufgaben auf die Gemeinschaftsebene wird die Stellung des Bundes gegenüber den Ländern gestärkt; denn soweit sich die Tätigkeit der Europäischen Gemeinschaften auf Gebiete erstreckt, die in die ausschließliche Kompetenz der Länder fallen, beschäftigen sich die Vertreter der Bundesregierung im Rat mit Angelegenheiten, bei denen der Bund im innerstaatlichen Verhältnis zu den Ländern keine Sachzuständigkeit besitzt. Das Ausgreifen der Gemeinschaften auf Kompetenzbereiche der Län-

119 Vgl. Haas $=$ Mitwirkung der Länder $=615$.

$120 \mathrm{Vgl}$. Rudolf $=$ Bundesländer und Gemeinschaftsrecht $=124$.

121 Rudolf $=$ Bundesländer und Gemeinschaftsrecht $=124$. 
der hat insofern auch eine Ausdehnung der Zuständigkeiten des Bundes zur Folge. "Für die Länder ergibt sich ein doppelter Erosionseffekt." 122

Um eine Stärkung der Rechte der Länder im europäischen Einigungsprozeß zu sichern, haben die Länder Nordrhein-Westfalen, Bayern, Hessen und Rheinland-Pfalz einen Antrag zur Änderung des Grundgesetzes eingebracht, wonach jede Übertragung von Hoheitsrechten auf zwischenstaatliche Einrichtungen nach Art. 24 GG nur mit Zustimmung des Bundesrates zulässig sein soll. ${ }^{123}$

Das europäische Gemeinschaftsrecht sieht prinzipiell keine Beteiligung von Gliedstaaten eines Mitgliedstaates am Rechtsetzungsprozeß der Gemeinschaft vor. Daher versuchen die Länder, eine stärkere Beteiligung am innerstaatlichen Willensbildungsproze $\beta$ zu erhalten. Schon in Art. 2 des Ratifikationsgesetzes zu den Römischen Verträgen (1957) wurde festgelegt, daß die Bundesregierung alle Vorschläge für Verordnungen, Richtlinien und Entscheidungen, die von der Kommission an das Ratssekretariat übermittelt werden, sowohl Bundestag als auch Bundesrat zuleiten muß. Diese Vorlagen werden in den Fachausschüssen beraten und das Ergebnis der Bundesregierung in Form einer Empfehlung übermittelt. Bis zum Abschluß der Einheitlichen Europäischen Akte blieb es allerdings dem Ermessen der Bundesregierung überlassen, inwieweit die Vertreter der Bundesregierung bei den Verhandlungen im Rat diese Empfehlungen beachteten. Im Gesetz zur Einheitlichen Europäischen Akte vom 28.2.1986 wurde dagegen festgelegt, daß die Bundesregierung von einer Stellungnahme, die ausschließliche Gesetzgebungsmaterien der Länder betrifft, nur aus unabweisbaren außen- und integrationspolitischen Gründen abweichen darf. "Im Falle einer Abweichung von der Stellungnahme des Bundesrates zu einer ausschließlichen Gesetzgebungsmaterie der Länder und im übrigen auf Verlangen, teilt die Bundesregierung die maßgeblichen Gründe mit."124 Allerdings ist "unabweisbar" ein unbestimmter Rechtsbegriff und die tatsächlichen Folgen für die Bundesregierung für den Fall, daß sie von der Stellungnahme der Länder abweicht, sind nicht ersichtlich.

Um der Informationsflut aus den Europäischen Gemeinschaften zu begegnen, hat der Bundesrat im Sommer 1988 eigens eine "EG-Kammer"125 eingerichtet,

122 Oschatz, Risse $=$ Deutscher Föderalismus $=10$.

$123 \mathrm{Vgl}$. =Gesetzesantrag der Länder $=$.

124 =Gesetz zur Einheitlichen Europäischen Akte $=1102$.

125 =Ergänzung der Geschäftsordnung des Bundesrates=. 
deren Beschlüsse die Wirkung von solchen des Bundesratsplenums haben sollen. ${ }^{126}$ Dadurch soll der Bundesrat schneller und flexibler auf die Entwicklung der Verhandlungssituation in Brüssel reagieren können. ${ }^{127}$ Der Bundesrat versucht so, seine politische Handlungsfähigkeit zu bewahren. Die Notwendigkeit dieser Maßnahme läßt sich daran ablesen, daß von den 1987 im Bundesratsplenum behandelten 503 Vorlagen 171 (also etwa ein Drittel) Vorlagen der Europäischen Gemeinschaften waren. 128

Auch in Anbetracht dieser stärkeren Einbeziehung des Bundesrates darf jedoch nicht übersehen werden, "daß der Bundesrat nicht ein allgemeines und umfassendes Repräsentativorgan der Länder ist. Die Länder werden in ihrem $\mathrm{Zu}$ ständigkeitsbereich keineswegs durch den Bundesrat mediatisiert." 129 Die Interessen der Länder gegenüber den Europäischen Gemeinschaften müssen nicht immer homogen sein, in Fragen des Zugangs zu Fördermitteln können durchaus auch Konkurrenzsituationen entstehen. ${ }^{130}$ Die Länder haben daher schon früh versucht, noch andere Instrumente der Einflußnahme zu schaffen.

Bereits während der Verhandlungen über die Römischen Verträge im Jahre 1956 wurde die Einrichtung des Beobachters der Länder bei den Europäischen Gemeinschaften geschaffen. Der Beobachter nimmt an den Ratssitzungen und an den vorbereitenden Sitzungen der Bundesministerien teil und wird von der Bundesregierung offiziell der deutschen Ratsdelegation zugeordnet. ${ }^{131}$ Obwohl seine Funktion als Informationsbeschaffungsstelle für die Länder nützlich ist, "ist seine personelle und technisch-administrative Ausstattung nicht so üppig, daß er eine zentrale Schaltstelle im EG-bezogenen Kommunikationsprozeß werden könnte ${ }^{132 " .}$

Die Länder suchten darüber hinaus verstärkt den direkten Kontakt zu den Brüsseler Institutionen. Dazu zählen einerseits Besuche von Mitgliedern der

126 "Im Unterschied zu den Ausschüssen des Bundesrates besitzt die EG-Kammer in EGFragen die gleichen Rechte und Kompetenzen wie das Plenum." Renzsch =Deutsche Länder und Europäische Integration $=31$.

$127 \mathrm{Vgl}$. Schweitzer $=$ Bundesstaat und $\mathrm{EG}=25$.

128 Vgl. o. V. =EG-Kammer $=5$.

129 Blumenwitz $=$ EG und Rechte der Länder $=218$.

$130 \mathrm{Vgl}$. Borchmann = Verbindungsbüros $=218$.

131 Vgl. dazu u. a. Hrbek = Doppelte Politikverflechtung = 26 und Blumenwitz =EG und Rechte der Länder $=222$.

132 Hrbek = Doppelte Politikverflechtung $=26$. 
Landesregierungen einschließlich der Ministerpräsidenten, andererseits aber auch die Einrichtung eigenständiger Länderbüros in Brüssel. 133 "Auf diese Art und Weise wird Information und Konsultation erreicht, die Möglichkeit der Beeinflussung der gemeinschaftlichen Rechtsetzung geschaffen und die politische Kontrolle der Bundesregierung erleichert." ${ }^{134}$ Allerdings sind diese Aktivitäten als "Nebenaußenpolitik" nicht unumstritten. ${ }^{135}$ Häufig wird eingewandt, daß dazu eine Kompetenz der Länder zur Pflege auswärtiger Beziehungen notwendig wäre. ${ }^{136}$

Daß die Länder in jüngster Zeit verstärkt in Entscheidungen der Europäischen Gemeinschaften einbezogen werden, zeigt sich daran, daß bei den Verhandlungen über die weitere Entwicklung der Europäischen Gemeinschaften im Dezember 1990 erstmals vier Länder mit offiziellem Delegationsstatus teilnehmen konnten. An der Regierungskonferenz über die politische Union nahmen Vertreter der Länder Baden-Württemberg und Nordrhein-Westfalen, an der Konferenz über die Wirtschafts- und Währungsunion Vertreter der Länder Bayern und Hamburg teil. ${ }^{137}$

Auch die Kommission ist den Ländern (und anderen regionalen Gebietskörperschaften in der Europäischen Gemeinschaft) entgegengekommen, indem sie im Juni 1988 einen "Beirat der regionalen und lokalen Gebietskörperschaften" einsetzte. ${ }^{138}$ Der Beirat besteht aus 42 Mitgliedern, die auf regionaler oder lokaler Ebene ein Wahlmandat innehaben. Die Mitglieder des Beirats werden von der Kommission auf Vorschlag der drei internationalen Verbände von Gebietskörperschaften (Versammlung der Regionen Europas, Internationaler Gemeindeverband und Rat der Gemeinden und Regionen Europas) ernannt. Der Beirat soll die Kommission in allen Fragen der regionalen Entwicklung, der Ausarbeitung und Durchfuihrung der europäischen Regionalpolitik, aber

133 Vgl. Hrbek = Doppelte Politikverflechtung = 32f. Im April 1989 hat das Land Hessen als letztes Land ein Informationsbüro für europäische Angelegenheiten offiziell eröffnet; vgl. o. V. =Europa-Büro $=6$.

134 Schweitzer $=$ Bundesstaat und $E G=22$.

$135 \mathrm{Vgl}$. dazu Borchmann $=$ Verbindungsbüros $=218 \mathrm{ff}$.

136 Vgl. Schweitzer $=$ Bundesstaat und $E G=22$; vgl. auch Hellwig $=$ Rolle der Bundesländer $=301 \mathrm{f}$.

137 Vgl. o. V. = Bundesländer $=2$.

138 Vgl. Kommission der EG =Einsetzung eines Beirats $1988=23 \mathrm{ff}$. 
auch in allen anderen Politikbereichen der Gemeinschaften mit regionalen und lokalen Auswirkungen beraten.

Diese Einrichtung ist insofern bemerkenswert, als sie die erste institutionelle Einbindung von Gebietskörperschaften der Mitgliedstaaten in ein Hilfsorgan darstellt und damit die eigenständigen Interessen dieser Gebietskörperschaften offiziell anerkannt wurden. Andererseits kann der Beirat für die Länder keine befriedigende Lösung bieten, weil durch die beschränkte Mitgliederzahl die meisten Länder überhaupt nicht im Beirat vertreten sind ${ }^{\mathbf{1 3 9}}$ und der Beirat nur beratende Funktion hat; "die Kommission ist in keiner Weise an dessen Äußerungen und Beschlüsse gebunden"140.

Aus den bisherigen Überlegungen läßt sich der Schluß ziehen, daß auch die These, die Länder verlören durch die europäische Integration am meisten an Bedeutung, zumindest relativiert werden muß. Die Länder haben durchaus die Möglichkeit, ihren Einfluß geltend zu machen, wenngleich dies häufig auf informellem Wege geschieht.

139 So sind für die Bundesrepublik sechs Sitze vorgesehen, wovon drei auf Vertreter der Länder und drei auf kommunale Vertreter entfallen. Vgl. Schweitzer =Bundesstaat und $\mathrm{EG}=23$.

140 Schweitzer $=$ Bundesstaat und $E G=23$. 


\section{Der Haushalt der Europäischen Gemeinschaften}

Bevor die Ausgabenverteilung und die Einnahmenverteilung in den Europäischen Gemeinschaften genauer betrachtet werden, sind zunächst einige grundsätzliche Bemerkungen zum Haushalt der Gemeinschaft erforderlich.

\section{Die Haushaltsinstrumente der Europäischen Gemeinschaften}

Vor dem Jahr 1968 stellte jede der drei Gemeinschaften eigene Haushaltspläne auf. Für die Europäische Gemeinschaft für Kohle und Stahl wurde ein Haushalt für die Verwaltungsausgaben (Art. 49 bis 56 EGKSV) und ein Funktionshaushaltsplan (Art. 78 bis $78 f$ EGKSV) aufgestellt. Der Vertrag zur Gründung der Europäischen Wirtschaftsgemeinschaft von 1957 sah für diese einen alle Einnahmen und Ausgaben umfassenden Haushalt vor (Art. 199 bis 209 EWGV), während der zur gleichen Zeit entstandene Vertrag zur Gründung der Europäischen Atomgemeinschaft einen Verwaltungshaushaltsplan sowie einen Forschungs- und Investitionshaushaltsplan für die Europäische Atomgemeinschaft vorschrieb (Art. 171 bis 183 EAGV).

Durch den Vertrag zur Einsetzung eines gemeinsamen Rates und einer gemeinsamen Kommission der Europäischen Gemeinschaften wurden der Verwaltungshaushaltsplan der Europäischen Gemeinschaft für Kohle und Stahl und die Haushaltspläne der Europäischen Atomgemeinschaft mit dem Haushaltsplan der Europäischen Wirtschaftsgemeinschaft verschmolzen. ${ }^{1}$ Zur Zeit gibt es daher im wesentlichen zwei Haushaltspläne: den Gesamthaushaltsplan der Europäischen Gemeinschaften und den Funktionshaushaltsplan der Europdischen Gemeinschaft für Kohle und Stahl. ${ }^{2}$

Die beiden Haushaltspläne wurden noch nicht zusammengefaßt, weil der Funktionshaushaltsplan der Europäischen Gemeinschaft für Kohle und Stahl einige besondere Merkmale aufweist; vor allem die Finanzierung mit Hilfe von Umlagen und Krediten unterscheidet ihn vom Gesamthaushaltsplan. Außerdem fiel im Rahmen der Europäischen Gemeinschaft für Kohle und Stahl die Entscheidungsbefugnis für den Haushalt von Beginn an der Hohen Behörde

1 Vgl. Art. 20 des Vertrages zur Einsetzung eines gemeinsamen Rates und einer gemeinsamen Kommission der Europäischen Gemeinschaften.

2 Auf die finanzielle Tätigkeit der Europäischen Investitionsbank wird hier nicht näher eingegangen, da sie eine rechtlich selbständige Institution darstellt. 
und damit jetzt der Kommission ${ }^{3}$ zu. Die Haushaltsbefugnisse der Europäischen Wirtschaftsgemeinschaft und der Europäischen Atomgemeinschaft lagen jedoch entsprechend den Römischen Verträgen zunächst ausschließlich in der Zuständigkeit des Ministerrates, der allein die Haushaltsbehörde darstellte; später wurde auch das Parlament an den Haushaltsbefugnissen beteiligt.

Nicht im Gesamthaushalt erfaßt werden außerdem die Tätigkeit des Europäischen Entwicklungsfonds und die Darlehens- und Anleihetätigkeit der Gemeinschaft. Über den Europäischen Entwicklungsfonds wird ein Großteil der finanziellen Entwicklungshilfe der Europäischen Gemeinschaften abgewickelt. ${ }^{4}$ Für einen Zeitraum von jeweils fünf Jahren wird ein bestimmtes Finanzvolumen zur Erfüllung von Entwicklungshilfeaufgaben festgesetzt. Solch ein FünfjahresFinanzvolumen wird 'Entwicklungsfonds' genannt. Der Europäische Entwicklungsfonds wird über Beiträge der Mitgliedstaaten finanziert. ${ }^{5}$ Diese Abweichung vom Grundsatz der Einheitlichkeit des Haushaltes ist politisch motiviert. Die Mitgliedstaaten wollen durch eine autonome Finanzregelung und über vom allgemeinen Recht abweichende Verwaltungsverfahren ihren direkten Einfluß auf bestimmte finanzielle Maßnahmen der Entwicklungshilfe erhalten.

Obwohl das Europäische Parlament und die Kommission seit langem die Einbeziehung des Europäischen Entwicklungsfonds in den Gesamthaushaltsplan gefordert haben und seit 1981 zwei den Europäischen Entwicklungsfonds betreffende Haushaltskapitel in den Gesamthaushaltsplan eingefügt wurden - allerdings wegen der fehlenden Rechtsgrundlage nur in Form eines Ansatzes "zur Erinnerung" (p. m.) -, einigte sich der Europäische Rat im Februar 1988 auf die Beibehaltung der abweichenden Finanzregelungen für den Europäischen Entwicklungsfonds und beide Kapitel wurden im Haushaltsplan 1989 wieder gestrichen. ${ }^{6}$

3 Nach Art. 9 des Vertrages zur Einsetzung eines gemeinsamen Rates und einer gemeinsamen Kommission der Europäischen Gemeinschaften wurden Rechte und Pflichten der Hohen Behörde seit 1967 durch die (gemeinsame) Kommission der EG wahrgenommen. Auf eine Umbenennung der Organe im Vertrag über die Gründung der Europäischen Gemeinschaft für Kohle und Stahl wurde jedoch - auch bei späteren Vertragsänderungen - verzichtet.

4 Vgl. Europäisches Parlament $=$ Haushaltsplan $1990=913$.

5 Vgl. Internes Abkommen über die Finanzierung und Verwaltung der Hilfe der Gemeinschaft im Rahmen des Vierten AKP-EWG-Abkommens.

6 Vgl. Kommission der EG $=$ Öffentliche Finanzen $=36$. 
Die Anleihe- und Darlehenstätigkeit der Europäischen Gemeinschaft für Kohle und Stahl und der Europäischen Atomgemeinschaft waren von vornherein in den Verträgen vorgesehen. ${ }^{7}$ Im Rahmen der Europäischen Gemeinschaft für Kohle und Stahl kann die Kommission Anleihen aufnehmen, sofern diese Mittel zur Gewährung von Krediten für die Finanzierung von Investitionen oder Umstellungsprogrammen im Kohle- und Stahlsektor verwendet werden. Die Aufnahme von Anleihen auf der Grundlage des Vertrages zur Gründung der Europäischen Atomgemeinschaft diente Zuschüssen zur Finanzierung von Kraftwerken.

Im Vertrag zur Gründung der Europäischen Wirtschaftsgemeinschaft waren Anleihe- und Darlehenstätigkeiten nicht ausdrücklich vorgesehen, seit 1975 werden sie allerdings für bestimmte Zwecke auf der Grundlage von Art. 235 EWGV genehmigt. Diese Zwecke sind die Stützung von Zahlungsbilanzen und die Förderung von Investitionen im Rahmen des sogenannten Neuen Gemeinschaftsinstruments. Durch dieses Instrument wird die Kommission ermächtigt, im Namen der Europäischen Wirtschaftsgemeinschaft Anleihen aufzunehmen, aus denen Darlehen für Investitionsvorhaben gewährt werden können, die zur industriellen Anpassung und zur Wettbewerbsfähigkeit der Gemeinschaft (insbesondere durch die Anwendung neuer Technologien und Innovationen) beitragen. ${ }^{8}$ Die gesamte Anleihe- und Darlehenstätigkeit der Gemeinschaft wird nicht im Haushalt erfaßt, es werden lediglich im Anhang einige Informationen dazu ausgewiesen. 9

\section{Das Haushaltsverfahren für den Gesamthaushalt der Europäischen Gemeinschaften}

Wie schon erwähnt, lag ursprünglich die Haushaltsbefugnis für den Gesamthaushalt allein beim Ministerrat. Im Jahre 1970 wurden die Haushaltsrechte geändert. ${ }^{10}$ Damals wurde die heute noch bedeutsame Einstufung der Ausgaben in obligatorische und sonstige, nicht-obligatorische Ausgaben eingeführt. Obligatorische Ausgaben sind solche, die sich zwingend aus den Verträgen

7 Vgl. Art. 49 EGKSV und Art. 172 Abs. 4 EAG.

8 Vgl. Rat der EG $=$ Neues Gemeinschaftsinstrument $=34 \mathrm{ff}$. $\mathrm{Zu}$ genaueren Erläuterungen vgl. Selmer $=$ Anleihekompetenzen $=21 \mathrm{ff}$.

9 Vgl. Kommission der EG =Öffentliche Finanzen $=38$.

10 Vgl. dazu Reister $=$ Haushalt der $\mathrm{EG}=120 \mathrm{ff}$. 
oder aus den aufgrund der Verträge erlassenen Rechtsakten ergeben. Für die Jahre 1971-1974 galten Übergangsvorschriften nach Art 203a EWGV: Das Parlament wurde ermächtigt, dem Rat Änderungen zum Entwurf des Haushaltsplans vorzuschlagen. Änderungsvorschläge, die keine Erhöhung des Gesamtbetrages der Ausgaben eines Organs zur Folge hatten - etwa dann, wenn Einsparungen an anderer Stelle vorgenommen wurden -, konnten nur durch eine qualifizierte Mehrheit des Rates abgelehnt werden. Kam ein Ablehnungsbeschluß nicht zustande, war der Änderungsvorschlag angenommen. ${ }^{11}$ Dieses sogenannte Prinzip der umgekehrten Mehrheit des Rates bedeutete einen ersten Schritt auf eine Mitentscheidung des Parlaments im Gegensatz zur Stellungnahme im Rahmen des alten Artikels 203.12

1975 wurden schließlich die wesentlichen gegenwärtigen Regelungen festgelegt. ${ }^{13}$ Jedes Organ der Gemeinschaft stellt vor dem 1. Juli einen Haushaltsvoranschlag für seine Ausgaben auf. Die Kommission faßt diese Voranschläge in einem Vorentwurf für den Haushaltsplan zusammen, der den Ansatz der Ausgaben und der Einnahmen enthält. ${ }^{14}$ Der Vorentwurf wird dem Rat vorgelegt, der auf dieser Grundlage mit qualifizierter Mehrheit einen Entwurf des Haushaltsplans beschließt und ihn an das Europäische Parlament weiterleitet. ${ }^{15}$ Nimmt das Parlament den Entwurf an, so ist der Haushaltsplan festgestellt. ${ }^{16}$ Lehnt das Parlament den Entwurf völlig ab, dann muß der Rat einen neuen Entwurf vorlegen. ${ }^{17}$ Das Parlament kann jedoch auch nur Änderungen am Haushalt vornehmen. Dann kommt die Unterscheidung zwischen obligatorischen und nicht-obligatorischen Ausgaben zum Tragen: Nicht-obligatorische Ausgaben können mit der Mehrheit der Mitglieder des Parlaments geändert werden, für die obligatorischen Ausgaben können nur Änderungsvorschläge gemacht werden, wobei die absolute Mehrheit der abgegebenen Stimmen erforderlich ist. 18

11 Vgl. Art. 203a Abs. 5 EWGV.

12 Vgl. Reister $=$ Haushalt der $E G=120$.

13 Vgl. Kommission der EG =Öffentliche Finanzen $=18$.

14 Vgl. Art. 203 Abs. 2 EWGV.

15 Vgl. Art. 203 Abs. 3 EWGV.

16 Vgl. Art. 203 Abs. 4 Satz 3 EWGV.

17 Vgl. Art. 203 Abs. 8 EWGV.

18 Vgl. Art. 203 Abs. 4 Satz 2 EWGV. 
Über den vom Parlament geänderten Haushalt befindet der Rat binnen 15 Tagen. Lehnt er innerhalb dieser Frist die Änderungen des Parlaments zu den nicht-obligatorischen Ausgaben und - soweit keine Erhöhung der Gesamtausgaben bewirkt wird - zu den obligatorischen Ausgaben nicht ab, so ist der Haushalt festgestellt. ${ }^{19}$ Hat der Rat seinerseits Änderungen an den Vorschlägen des Parlaments zu den nicht-obligatorischen Ausgaben beschlossen oder die Vorschläge zu den obligatorischen Ausgaben abgelehnt, dann wird das Parlament erneut befaßt. ${ }^{20}$ Es kann jedoch nur noch über die nicht-obligatorischen Ausgaben bestimmen. Binnen 15 Tagen kann es mit Mehrheit der Stimmen in letzter Instanz über den Umfang dieser Ausgaben entscheiden. Damit stellt es schließlich auch den Haushaltsplan fest. ${ }^{21}$

Die Befugnis des Europäischen Parlaments, Abänderungen bei den nicht-obligatorischen Ausgaben vorzunehmen, wird allerdings durch einen sogenannten Höchstsatz begrenzt, der sich aus verschiedenen Indikatoren ergibt, z. B. der Entwicklung des Sozialproduktes der Gemeinschaft und der durchschnittlichen Veränderung der Haushaltspläne der Mitgliedstaaten. ${ }^{22}$ Dieser Höchstsatz kann im Einvernehmen zwischen Rat und Europäischem Parlament grundsätzlich erhöht werden. Für die Jahre 1988 bis 1992 sollten gemäß der interinstitutionellen Vereinbarung zwischen Rat und Parlament die Höchstsätze akzeptiert werden. ${ }^{23}$ Die tatsächlichen Steigerungsraten der nicht-obligatorischen Ausgaben lagen jedoch in diesen Jahren stets über dem festgelegten Höchstsatz. ${ }^{24}$

19 Vgl. Art. 203 Abs. 5 Satz 3 EWGV.

20 Vgl. Art. 203 Abs. 5 Satz 4 EWGV.

21 Vgl. Art. 203 Abs. 6 EWGV.

22 Vgl. dazu Art. 203 Abs. 9 EWGV. Zu den Höchstsätzen der Jahre 1975 bis 1989 vgl. Kommission der EG =Öffentliche Finanzen $=68$.

23 Vgl. Rat der EG = Interinstitutionelle Vereinbarung 1988= 33ff., hier insbesondere S. 35.

24 Vgl. Bundesministerium der Finanzen $=$ EG-Haushalt 1990= Anlage 8. 


\section{Die Ausgabenverteilung}

Ebenso wie die Aufgabenverteilung war auch die Ausgabenverteilung in den Europäischen Gemeinschaften Wandlungen unterworfen, jedoch in weit geringerem Maße. Dies mag daran liegen, daß viele Politikbereiche (z. B. die Rechtsangleichung im Hinblick auf den Binnenmarkt) nur in Form einer Rahmensetzung von den Europäischen Gemeinschaften erfüllt werden. Die Frage, welche Aufgaben tatsächlich auch Ausgaben für die Europäischen Gemeinschaften nach sich ziehen und welche Konsequenzen sich daraus für die Ausgaben der nationalen Ebenen ergeben, ist Inhalt des folgenden Abschnitts.

\section{Die Ausgaben der Europäischen Gemeinschaften}

\section{a) Überblick}

Während sich die Ausgaben im ersten Jahr des Gesamthaushaltes der Gemeinschaft 1968 nur auf knapp fünf Mrd. DM beliefen, ${ }^{1}$ stiegen sie bis 1989 auf 88,5 Mrd. DM an. Der Haushalt der Europäischen Gemeinschaften hat dennoch zur Zeit eine Größenordnung von nicht mehr als ca. 3\% der Haushalte aller Mitgliedstaaten zusammen oder rund $1 \%$ ihres gesamten Bruttosozialproduktes. $^{2}$ Sein Volumen umfaßt jetzt knapp ein Drittel des Bundeshaushalts (Tabelle 2). Seit 1983 ist der Haushalt der Europäischen Gemeinschaften jedoch größer als der Haushalt des größten Bundeslandes, Nordrhein-Westfalen.

Der Haushalt der Europäischen Gemeinschaften gliedert sich nach den Organen Europäisches Parlament, Ministerrat, Kommission, Europäischer Gerichtshof und Europäischer Rechnungshof in fünf Einzelpläne. Der Hauptanteil der Ausgaben (1991 ca. 98\%) entfällt dabei auf die Kommission, "da sie mit der Finanzierung und Durchführung der Gemeinschaftspolitik beauftragt ist; bei allen übrigen Organen fallen fast ausschließlich Verwaltungsausgaben $a^{\prime \prime 3}$. Die Verwaltungsausgaben aller Organe machen ca. 5\% der Gesamtausgaben aus. Die Ausgaben für die Finanzierung der Gemeinschaftspolitik (operationell verwendete) Mittel haben einen Anteil von ca. 95\% an den Gesamt-

$1 \quad$ Vgl. = Finanzen der $\mathrm{EG}=799$.

2 Vgl. Kommission der EG =Finanzbericht $1988=6$.

$3 \quad$ Nottelmann $=$ Haushalt der $\mathrm{EG}=52$. 
Tabelle 2: Die Ausgaben des Gesamthaushaltes der Europäischen Gemeinschaften im Vergleich zu den Ausgaben der nationalen Ebenen in der Bundesrepublik Deutschland (in Mrd. DM)

\begin{tabular}{|c|c|c|c|c|c|c|c|c|c|c|}
\hline & 1981 & 1982 & 1983 & 1984 & 1985 & 1986 & 1987 & 1988 & 1989 & 1990 \\
\hline Ausgaben der EG & 44,7 & 48,5 & 55,2 & 61,6 & 62,6 & 72,8 & 73,2 & 85,5 & 88,5 & 90,8 \\
\hline $\begin{array}{l}\text { Ausgaben des Bundes }{ }^{1)} \\
\text { Ausgaben der Länder }\end{array}$ & 233,0 & 244,6 & 246,7 & 251,8 & 257,1 & 261,5 & 269,0 & 275,3 & 289,9 & 380,2 \\
\hline $\begin{array}{l}\text {-Schleswig-Holstein } \\
\text {-Niedersachsen } \\
\text {-Nordrhein-Westfalen } \\
\text {-Hessen } \\
\text {-Rheinland-Pfalz } \\
\text {-Baden-Württemberg } \\
\text {-Bayern } \\
\text {-Saarland } \\
\text {-Hamburg } \\
\text {-Bremen } \\
\text {-Berlin }\end{array}$ & $\begin{array}{r}8,1 \\
23,1 \\
43,1 \\
16,9 \\
11,6 \\
30,3 \\
33,0 \\
3,5 \\
10,6 \\
4,6 \\
17,3\end{array}$ & $\begin{array}{r}8,4 \\
24,3 \\
55,2 \\
17,5 \\
11,9 \\
30,9 \\
34,2 \\
3,9 \\
11,2 \\
4,7 \\
17,9\end{array}$ & $\begin{array}{r}8,9 \\
24,4 \\
54,6 \\
17,8 \\
12,2 \\
31,7 \\
34,8 \\
4,0 \\
11,7 \\
4,8 \\
18,6\end{array}$ & $\begin{array}{r}8,9 \\
25,0 \\
55,5 \\
18,6 \\
12,4 \\
33,0 \\
35,5 \\
4,1 \\
11,9 \\
4,9 \\
19,4\end{array}$ & $\begin{array}{r}9,3 \\
26,3 \\
56,5 \\
19,6 \\
13,0 \\
33,7 \\
37,4 \\
4,7 \\
12,2 \\
5,3 \\
20,3\end{array}$ & $\begin{array}{r}9,7 \\
27,9 \\
58,4 \\
20,8 \\
13,6 \\
35,2 \\
38,7 \\
4,3 \\
12,6 \\
5,2 \\
20,7\end{array}$ & $\begin{array}{r}10,3 \\
28,0 \\
60,1 \\
21,7 \\
14,1 \\
37,1 \\
39,8 \\
4,5 \\
12,9 \\
5,5 \\
21,6\end{array}$ & $\begin{array}{r}10,5 \\
28,3 \\
60,8 \\
22,4 \\
14,4 \\
38,6 \\
41,0 \\
4,7 \\
127 \\
5,8 \\
22,3\end{array}$ & $\begin{array}{r}10,9 \\
29,7 \\
62,9 \\
23,6 \\
15,2 \\
40,1 \\
42,9 \\
5,0 \\
13,0 \\
6,1 \\
23,8\end{array}$ & $\begin{array}{r}11,5 \\
31,2 \\
67,4 \\
25,2 \\
16,3 \\
43,4 \\
45,2 \\
6,4 \\
14,1 \\
6,4 \\
24,8\end{array}$ \\
\hline Länder insgesamt ${ }^{1)}$ & 210,6 & 217,6 & 221,1 & 226,7 & 235,1 & 244,0 & 252,3 & 257,9 & 269,5 & 286,1 \\
\hline $\begin{array}{l}\text { Ausgaben der } \\
\text { Gemeinden } 1 \text { ) }\end{array}$ & 152,1 & 153,0 & 151,7 & 154,7 & 162,9 & 172,5 & 179,2 & 184,4 & 193,1 & 208,1 \\
\hline
\end{tabular}

Quelle: Bundesministerium der Finanzen =Finanzbericht $1987=242$, =Finanzbericht $1991=133$ und $294 \mathrm{f}$. und $=$ Finanzbericht $1992=127,155$ und $286 f$. 
Tabelle 3: Übersicht über die Ausgaben des Gesamthaushaltes der Europäischen Gemeinschaften (in Mio. ECU)

\begin{tabular}{|c|c|c|c|c|c|c|c|c|}
\hline & 1985 & 1986 & 1987 & 1988 & 1989 & 1990 & $1991^{*}$ & $1992^{*}$ \\
\hline Parlament (EP I) & 228,5 & 287,3 & 322,3 & 369,9 & 378,2 & 429,7 & 512,2 & 588,8 \\
\hline Rat (EP II) & 148,4 & 177,4 & 194,2 & 203,7 & 227,9 & 279,8 & 350,0 & 347,1 \\
\hline $\begin{array}{l}\text { Kommission (EP III) } \\
\text { davon Verwaltungsmittel } \\
\text { davon operationelle Mittel: } \\
\text {-EAGFL Garantie (Titel 1 und 2) } \\
\text {-Agrarstrukturen (Titel 3) } \\
\text {-Fischerei- und Seepolitik (Titel 4) } \\
\text {-Regionalentwicklung, Verkehr (Titel 5) } \\
\text {-Sozialpolitik (Titel 6) } \\
\text {-Energie- Industrie-. Technologie- }\end{array}$ & $\begin{array}{r}27672,2 \\
869,5 \\
26802,7 \\
19725,9 \\
738,6 \\
81,8 \\
1725,5 \\
1490,7\end{array}$ & $\begin{array}{r}33669,4 \\
998,6 \\
32670,8 \\
22120,0 \\
766,5 \\
115,8 \\
2585,1 \\
2419,2\end{array}$ & $\begin{array}{r}34739,3 \\
1098,5 \\
33641,0 \\
22951,8 \\
888,6 \\
157,8 \\
2687,3 \\
2852,5\end{array}$ & $\begin{array}{r}40632,9 \\
1253,8 \\
39379,1 \\
26389,6 \\
1194,6 \\
260,0 \\
3348,4 \\
2501,3\end{array}$ & $\begin{array}{r}40441,6 \\
1362,3 \\
39073,3 \\
24460,4 \\
1413,1 \\
261,9 \\
4144,3 \\
2964,1\end{array}$ & $\begin{array}{r}42692,1 \\
1510,0 \\
41182,1 \\
225069,1 \\
2075,3 \\
305,9 \\
4922,5 \\
3226,2\end{array}$ & $\begin{array}{r}55114,6 \\
1719,4 \\
53395,2 \\
32419,0 \\
2572,9 \\
379,1 \\
6861,5 \\
4091,9\end{array}$ & $\begin{array}{r}61772,9 \\
1872,7 \\
59900,2 \\
36022,0 \\
3225,6 \\
351,5 \\
9477,8 \\
4834,1\end{array}$ \\
\hline & 707,5 & 838,4 & 900,3 & 1196,5 & 1497,3 & 1817,59 & 1812,4 & 2373,7 \\
\hline $\begin{array}{l}\text { gliedstaaten (Titel 8) } \\
\text {-Zusammenarbeit mit Entwicklungs- }\end{array}$ & 1248,0 & 2972,4 & 2408,9 & 3447,4 & 3268,5 & 2393,7 & 1740,2 & 1150,6 \\
\hline ländern und Drittländern (Titel 9) & 1084,7 & 853,4 & 793,8 & 1041,3 & 1063,8 & 1816,7 & 2008,9 & 2486,7 \\
\hline $\begin{array}{l}\text { Gerichtshof (EP IV) } \\
\text { Rechnungshof (EP V) }\end{array}$ & $\begin{array}{l}31,1 \\
18,5\end{array}$ & $\begin{array}{l}37,1 \\
21,6\end{array}$ & $\begin{array}{l}43,9 \\
24,5\end{array}$ & $\begin{array}{l}46,7 \\
25,7\end{array}$ & $\begin{array}{l}54,8 \\
28,4\end{array}$ & $\begin{array}{l}66,0 \\
53,9\end{array}$ & $\begin{array}{l}75,1 \\
33,5\end{array}$ & $\begin{array}{l}82,1 \\
36,7\end{array}$ \\
\hline Summe & 28098,7 & 34192,8 & 35324,4 & 41278,9 & 41125,0 & 43521,5 & 56085,4 & 62827,6 \\
\hline
\end{tabular}

* = Haushaltsansätze (Soll-Größen)

Seit 1991 hat sich die Einteilung der Titel im Gesamthaushalt geändert. Die Einzelpläne der Organe wurden zunächst in Teileinzelpläne unterteilt und erst danach in Titel gegliedert; zum Teil wurden neue Abgrenzungen der Titel eingeführt. Um die Einheitlichkeit zu wahren, wurde die neue an die frühere Einteilung angepaßt.

Quelle: Europäischer Rechnungshof =Jahresbericht $1988=226$, =Jahresbericht $1989=72$ und Europäisches Parlament $=$ Haushaltsplan $1991=$ und $=$ Haushaltsplan $1992=$. 


\section{Ausgabenverteilung}

ausgaben. Die Verteilung der Mittel auf die einzelnen Titel kann Tabelle 3 entnommen werden.

Im folgenden sollen die großen Ausgabenkategorien, die sich im Haushalt unterscheiden lassen, näher betrachtet werden.

\section{b) Die Ausgaben für die Garantie der Agrarmärkte}

Den größten Anteil an den Gesamtausgaben der Europäischen Gemeinschaften haben seit Jahren die Ausgaben für die Garantie der Agrarmärkte. Die Ausgaben für das Agrarmarktordnungssystem werden im Rahmen des 1962 gegründeten Europäischen Ausgleichs- und Garantiefonds für die Landwirtschaft, Abteilung Garantie, ausgewiesen. Der Europäische Ausgleichs- und Garantiefonds für die Landwirtschaft ist kein Fonds im eigentlichen Sinne, da er weder über eine eigene Rechtsfähigkeit noch über Finanzautonomie verfügt. Er war bis 1967 Bestandteil des Haushaltes der Europäischen Wirtschaftsgemeinschaft und ist seither in den Gesamthaushalt integriert. Seine Ausgaben werden grundsätzlich nach dem Non-Affektationsprinzip 4 aus den gesamten dem Gemeinschaftshaushalt zur Verfügung stehenden Einnahmen finanziert. ${ }^{5}$

Der größte Teil der landwirtschaftlichen Erzeugnisse unterliegt dem gemeinsamen Marktordnungssystem, das unmittelbar der Stabilisierung der Preise für diese Erzeugnisse dient, wobei das zu stabilisierende Preisniveau in der Regel höher liegt als die entsprechenden Weltmarktpreise. Der Ministerrat legt alljährlich einheitliche Richtpreise für die einzelnen Marktordnungsprodukte fest. Diese Richtpreise haben für das Instrumentarium der gemeinsamen Agrarpolitik große Bedeutung:

1. Die Gemeinschaft ist zu Interventionskäufen im Binnenmarkt gezwungen, wenn die heimische Nachfrage nicht ausreicht, um die heimische Produktion voll zu absorbieren. Aus den Richtpreisen werden sogenannte Interventionspreise abgeleitet, zu denen die Interventionsstellen zum Ankauf der ihnen angebotenen Erzeugnisse verpflichtet sind und die damit eine Mindestpreisgarantie darstellen.

4 Vgl. zum Non-Affektationsprinzip auch Abschnitt D. III. 2. a).

$5 \quad$ Vgl. Ott $=$ Verteilungswirkungen $=151$. 
2. Durch eine variable Importausgleichsabgabe werden Importe aus Drittstaaten auf das Binnenpreisniveau angehoben.

3. Bei der Ausfuhr von Agrarprodukten in Drittstaaten ist das Gemeinschaftspreisniveau Bestimmungsgröße für die Höhe der Exporterstattungen, die die Differenz zu den Weltmarktpreisen ausgleichen.

Nach der Art der agrarpolitischen Maßnahmen lassen sich Erstattungen bei der Ausfuhr und Interventionen im Binnenmarkt unterscheiden. Das Schwergewicht der Ausgaben liegt auf den Binnenmarktinterventionen, die ungefähr zwei Drittel der Ausgaben beanspruchen, während ungefähr ein Drittel auf die Ausfuhrerstattungen entfällt. ${ }^{6}$ Bei den Binnenmarktinterventionen haben neben den Ankäufen von Agrarprodukten und der Lagerhaltung vor allem die Beihilfen zum Preisausgleich großes Gewicht. Diese werden entweder als Erzeugerhilfen oder als Verarbeitungshilfen gewährt. Erstere werden an die Produzenten direkt bezahlt und stellen für sie ein zusätzliches Einkommen im Sinne einer Transferzahlung dar, während die Beihilfen zur Verarbeitung der Grunderzeugnisse eine Absatzgarantie bewirken. ${ }^{7}$

Vom Europäischen Ausgleichs- und Garantiefonds für die Landwirtschaft, Abteilung Garantie, wird außerdem das 1988 vom Agrarministerrat beschlossene Programm zur Förderung von Flächenstillegungen mitgetragen. ${ }^{8}$ Die Ausgaben, die im Rahmen dieses Programms von der Gemeinschaft getragen werden müssen, werden je zur Hälfte von der Abteilung Garantie und der Abteilung Ausrichtung des Agrarfonds finanziert.

Der hohe Anteil der Abteilung Garantie des Agrarfonds am Gesamtbetrag der Ausgaben wird in Tabelle 4 deutlich. Bis 1988 lag der Anteil mit Schwankungen bei etwa zwei Drittel der Gesamtausgaben, seit 1989 scheint sich eine Senkung des Anteils durchzusetzen. Gleichwohl ist der Anteil mit fast $60 \%$ noch immer sehr hoch. Außerdem muß beachtet werden, daß für die Jahre 1990 und 1991 nur Haushaltsansätze, nicht aber tatsächliche Werte angegeben werden können.

6 Vgl. Kommission der EG =Lage der Landwirtschaft 1990= Tabellenteil 88f. und eigene Berechnungen.

$7 \quad$ Vgl. dazu auch Ott $=$ Verteilungswirkungen $=154$.

8 Vgl. Rat der EG =Stillegung von Ackerflächen $1988=28 \mathrm{ff}$. 
Tabelle 4: Anteil der jährlichen Ausgaben der Abteilung Garantie des Europäischen Ausgleichs- und Garantiefonds für die Landwirtschaft am Gesamtbetrag der Ausgaben (Jährliche Haushaltsausgaben $=100 \%$ )

\begin{tabular}{|llllllllll|}
\hline 1980 & 1981 & 1982 & 1983 & 1984 & 1985 & 1986 & 1987 & 1988 & 1989 \\
69,4 & 61,6 & 60,6 & 64,9 & 66,6 & 70,2 & 64,7 & 65,0 & 64,9 & 59,9 \\
\hline & & & & & & & & & \\
$1990^{*}$ & $1991^{*}$ \\
58,6 & 58,4 & & & & & & & & \\
\hline
\end{tabular}

Quelle: Europäischer Rechnungshof =Jahresbericht $1988=$ und eigene Berechnungen.

\section{c) Die Ausgaben für die Strukturpolitik der Gemeinschaft}

\section{aa) Die Ausgaben der Strukturfonds}

Während die Marktordnungsausgaben durch die Abteilung Garantie des Agrarfonds voll finanziert werden, ergänzen die Strukturfonds lediglich mitgliedstaatliche Aktivitäten. Je nach Art der getroffenen Maßnahme kommen bei den Interventionen der Strukturfonds unterschiedliche Finanzierungsformen zum Einsatz. ${ }^{9}$ Die wichtigste Form ist die Kofinanzierung sogenannter operationeller Programme; diese werden von den Mitgliedstaaten entworfen und durchgeführt, aber von der Gemeinschaft mitfinanziert. ${ }^{10}$ Daneben gibt es jedoch auch Globalzuschüsse, die von einer vom Mitgliedstaat bezeichneten, zwischengeschalteten Stelle verwaltet und von dieser in Form von Einzelzuschüssen an Endbegünstigte weitergeleitet werden. Damit sollen insbesondere unternehmerische Initiativen gefördert werden. Schließlich muß noch die Kofi-

9 Vgl. dazu im folgenden Kommission der EG = Reform der Strukturfonds 1989= $33 \mathrm{ff}$.

10 Früher nannte man entsprechende Programme "nationale Programme von gemeinschaftlichem Interesse". 
nanzierung von Einzelprojekten (insbesondere von Infrastrukturmaßnahmen) erwähnt werden. Die Finanzierung von Maßnahmen im Rahmen der Strukturfonds erfolgt in enger Zusammenarbeit zwischen der Kommission und den Behörden der Mitgliedstaaten.

Die Strukturfonds spielten bisher im Vergleich zur Abteilung Garantie des Agrarfonds eine wesentlich geringere Rolle. Ihr Anteil an den Gesamtausgaben lag für die einzelnen Fonds unter 10\%; in den letzten Jahren hat sich der Anteil jedoch deutlich vergrößert (siehe Tabelle 5). Das erklärte Ziel der Europäischen Gemeinschaften ist es, die Mittel der Strukturfonds bis 1993 gegenüber dem Stand von 1987 real zu verdoppeln.

Tabelle 5: Der Anteil der Strukturfonds an den Gesamtausgaben in den Jahren 1975 bis 1991 (in \%)

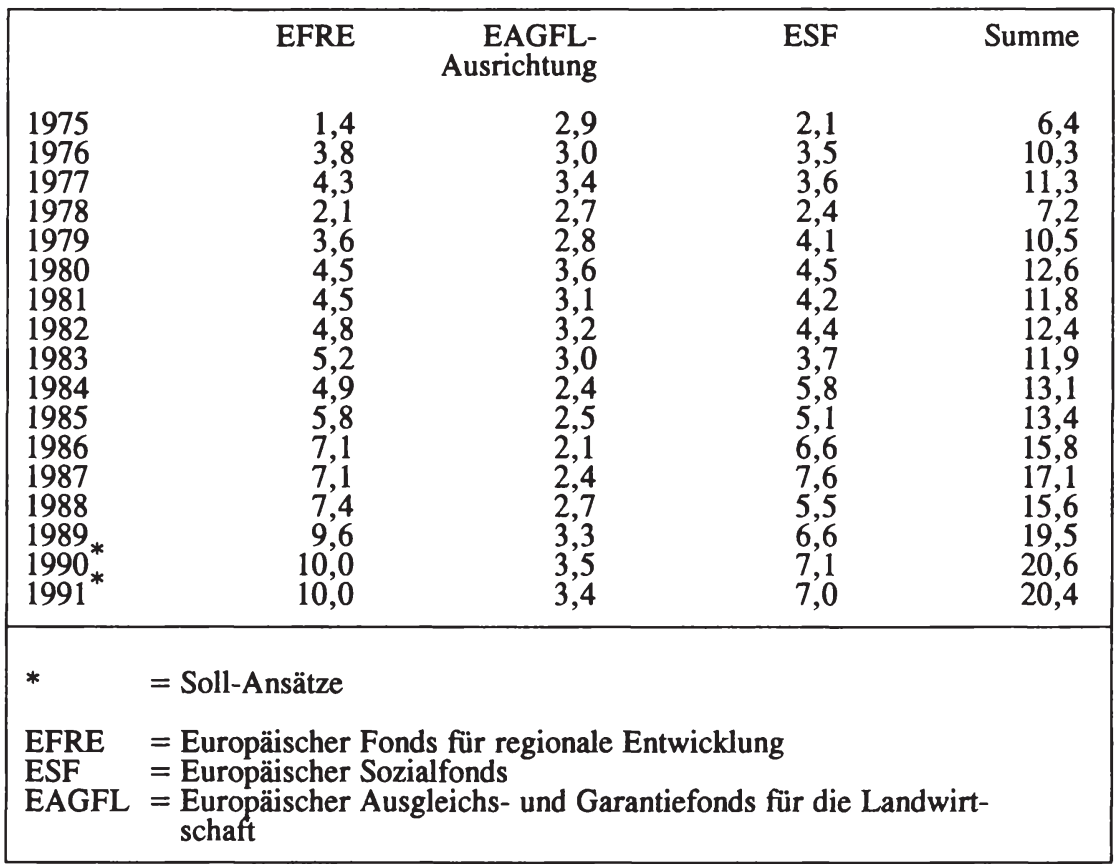

Quelle: Europäischer Rechnungshof =Jahresbericht 1989= und Europäisches Parlament $=$ Haushaltsplan 1991 = sowie eigene Berechnungen. 


\section{Ausgabenverteilung}

Die Verteilung der Strukturfondsmittel auf die festgelegten Ziele wird in Tabelle 6 deutlich. Der größte Teil der Strukturfondsmittel (fast zwei Drittel) wird für das Ziel Förderung der Entwicklung und der strukturellen Anpassung der Regionen mit Entwicklungsrückstand (Ziel Nr. 1) verwendet, an dem alle drei Fonds beteiligt sind. Die Ziele Nr. 3 und 4 (also die arbeitsmarktpolitischen Ziele), die nur vom Europäischen Sozialfonds getragen werden, nehmen mit einem deutlich geringeren Betrag die zweite Position ein, gefolgt von Ziel Nr. 2 (Umstellung der Regionen, die von rückläufiger industrieller Entwicklung stark betroffen sind). Der geringste Teil der Mittel fließt den agrarpolitischen Zielen (Nr. 5a und 5b) zu.

Tabelle 6: Die Aufteilung der Strukturfondsmittel auf die einzelnen Ziele im Jahr 1989 (in Mio. ECU)

\begin{tabular}{|lrrrr|}
\hline & $\begin{array}{r}\text { EAGFL-Aus- } \\
\text { richtung }\end{array}$ & EFRE & ESF & Summe \\
Ziel Nr. 1 & 862 & 3.630 & 1.645 & 6.137 \\
Ziel Nr. 2 & - & 758 & 302 & 1.060 \\
Ziele Nr. 3+4 & $51 \overline{6}$ & - & 1.332 & 1.332 \\
Ziel 5a & 27 & 115 & 90 & 2316 \\
Ziel 5b & 57 & 164 & 109 & 330 \\
Ubergangsmaß- & & & & \\
nahmen u. ä. & & & & \\
\hline EFRE = Europäischer Fonds für regionale Entwicklung & \\
ESF = Europäischer Sozialfonds \\
EAGFL = Europäischer Ausgleichs- und Garantiefonds für die Landwirt- \\
schaft
\end{tabular}

Quelle: Kommission der EG $=$ Durchführung der Strukturfonds $=94$.

\section{bb) Sonstige Ausgaben mit struktureller Zweckbestimmung}

Die Gemeinschaft finanziert noch eine Reihe weiterer struktureller Maßnahmen, allerdings mit deutlich bescheidenerem Finanzaufwand. ${ }^{11} \mathrm{Zu}$ nennen sind hier die integrierten Mittelmeerprogramme, ${ }^{12}$ das Programm zur

11 Vgl. dazu auch Kommission der EG =Öffentliche Finanzen $=66$.

$12 \mathrm{Zu}$ diesen mehrjährigen Programmen vgl. Rat der EG =Integrierte Mittelmeerprogramme $1985=1 \mathrm{ff}$. 
Förderung der industriellen Entwicklung Portugals, ${ }^{13}$ die gemeinsame Fischerei- und Seepolitik, ${ }^{14}$ bestimmte Ausgaben im Bereich der Landwirtschaft, die nicht im Rahmen des Europäischen Ausgleichs- und Garantiefonds für die Landwirtschaft abgewickelt werden, 15 Maßnahmen für den Verkehr und den Fremdenverkehr, 16 Ausgaben auf dem Gebiet der allgemeinen und der beruflichen Bildung und der Jugendpolitik, Ausgaben für Beschäftigung, sozialen Schutz, Gesundheit und Katastrophenhilfe. ${ }^{17}$ Diese Ausgaben machen jedoch zusammen nur ca. $2,5 \%$ der Gesamtausgaben aus.

\section{d) Ausgaben in den Bereichen Energie, Industrie, Technologie und For- schung}

Die Ausgaben in den Bereichen Energie, Industrie, Technologie und Forschung wurden bis 1990 in Titel 7 des Haushaltsplans der Europäischen Gemeinschaften eingestellt. Seit 1991 setzt man diese Ausgaben in drei getrennten Teileinzelplänen an. In einem ersten werden die Ausgaben für die Energiepolitik, die Sicherheitsüberwachung im Rahmen der Europäischen Atomgemeinschaft und umweltpolitische Maßnahmen zusammengefaßt. Diese Ausgaben haben lediglich einen Anteil von unter einem Prozent an den Gesamtausgaben. Ein weiterer Teileinzelplan umfaßt Maßnahmen zum Verbraucherschutz, Hilfen für den Wiederaufbau nach Katastrophenfällen, Aktionen im Bereich des Binnenmarktes, Maßnahmen auf dem Gebiet der Informationstechnologien und der Telekommunikation, Maßnahmen im Bereich der gewerblichen Wirtschaft sowie Ausgaben für statistische Informationen. Auch der Anteil dieser Ausgaben an den Gesamtausgaben ist sehr gering. In bedeutend höherem Umfang fließen Mittel in den dritten Teileinzelplan "Forschung und technologische Entwicklung". Hier werden die Ausgaben für die gemeinsame Forschungsstelle und die Ausgaben, die sich aus der Beteiligung der Europäischen Gemeinschaft an Forschungsprojekten ergeben, veranschlagt. Insgesamt macht dieser $\mathrm{Be}-$ reich ca. 3,5\% der Gesamtausgaben aus.

13 Vgl. Rat der EG = Verordnung über PEDIP 1988= 21ff.

14 Vgl. Titel B2 Kapitel 9 des Einzelplans der Kommission.

15 Z. B. Programme zur Tilgung von Seuchen in der EG und Kontroll- und Betrugsbekämpfungsmaßnahmen.

16 Vgl. Titel B2 Kapitel 7 des Einzelplans der Kommission.

17 Im Haushaltsplan 1991 wurden diese Ausgaben erstmals von den Strukturmaßnahmen getrennt und in einem gesonderten Teileinzelplan "Sozialmaßnahmen" ausgewiesen. 


\section{Ausgabenverteilung}

\section{e) Erstattungen an Mitgliedstaaten}

Titel 8 des Einzelplans der Kommission ${ }^{18}$ umfaßt vor allem die Erstattungen für die "neuen" Mitgliedstaaten. ${ }^{19}$ Die Beitrittsakte Spaniens und Portugals ${ }^{20}$ sah in Art. 187 und Art. 374 vor, daß diesen beiden Staaten zeitlich befristet ${ }^{21}$ ein Teil ihrer Zahlungen an die Europäischen Gemeinschaften ${ }^{22}$ erstattet werden. Es wurde davon ausgegangen, daß neue Mitgliedstaaten erst nach einigen Jahren der Zugehörigkeit zu den Europäischen Gemeinschaften in den vollen Genuß der Mitgliedschaft kämen und insofern eine volle Leistungspflicht von Anfang an nicht angemessen sei. ${ }^{23}$ Anstatt von vornherein weniger Einnahmen zu erheben, wird dieser Ausgleich auf der Ausgabenseite durchgeführt. Vergleichbare Erstattungen hatten bei ihrem Beitritt auch Großbritannien, Irland, Dänemark und Griechenland erhalten. Im Jahre 1989 hatte Spanien noch Erstattungen in Höhe von 1.023,3 Mio. ECU und Portugal in Höhe von 120,0 Mio. ECU erhalten. Für 1991 sind entsprechend der Degression nur noch 360,7 Mio. ECU für Spanien und 38,5 Mio. ECU für Portugal vorgesehen. ${ }^{24}$

18 Seit 1991 Titel 11 (Erstattungen und Ausgleichszahlungen) des Teileinzelplans B0 der Kommission. Vgl. Anmerkung zu Tabelle 3.

19 Bis 1988 wurden hier auch die Erstattungen an die Mitgliedstaaten für die Erhebung der eigenen Mittel der Europäischen Gemeinschaft in Höhe von 10\% der Mittel eingestellt. Seit 1988 wird dieser Betrag jedoch direkt von den Einnahmen abgezogen. Vgl. dazu genauer B. III. 2. a).

20 Vgl. Akte über den Beitritt des Königreichs Spanien und der Portugiesischen Republik und die Anpassung der Verträge, in: Amtsblatt der EG, Nr. L 302, vom 15.11.1985.

21 Die Befristung läuft bis 1991. Die Prozentsätze der Erstattungen sind degressiv gestaffelt:

1986: $87 \%$

1987: $70 \%$

1988: $55 \%$

1989: $40 \%$

1990: $25 \%$

1991: $5 \%$.

22 Dies bezog sich zunächst auf die Mehrwertsteuer-Zahlungen; 1988 wurden die Erstattungen auf die Bruttosozialprodukt-Eigenmittel ausgedehnt. Seit 1988 erhalten Spanien und Portugal auch Erstattungen für ihre Beiträge zur Finanzierung des Ausgleichs für Großbritannien; vgl. dazu II. C. 2.

23 Vgl. Akte über die Bedingungen des Beitritts des Königreichs Spanien und der Portugiesischen Republik und die Anpassung der Verträge, in: Amtsblatt der EG, Nr. L 302, vom 15.11.1985, S. 23.

24 Vgl. Europäisches Parlament $=$ Haushaltsplan $1991=1316$. 


\section{f) Ausgaben für die Zusammenarbeit mit Entwicklungsländern und Drittländern}

Die Ausgaben für die Zusammenarbeit mit Entwicklungsländern und Drittländern umfassen vor allem Nahrungsmittelhilfen in Form von Getreide, Milcherzeugnissen, Zucker, Olivenöl etc., die finanzielle und technische Zusammenarbeit mit Entwicklungsländern in Asien und Lateinamerika, Hilfe für Flüchtlinge und Vertriebene, Katastrophenhilfe für Entwicklungsländer, aber auch Ausgaben für die Zusammenarbeit mit den Staaten des Mittelmeerraumes.

Wie bereits erwähnt, spiegelt jedoch der Mittelansatz im Haushalt nicht die tatsächliche Bedeutung der Entwicklungspolitik der Gemeinschaft wider, da die gesamte Politik der Zusammenarbeit mit bestimmten Staaten Afrikas, der Karibik und des Pazifischen Raums (in den Dokumenten der Europäischen Gemeinschaften AKP-Staaten genannt) ${ }^{\mathbf{2 5}}$ außerhalb des Haushaltes über den Europäischen Entwicklungsfonds finanziert wird. Dabei fallen nochmals Ausgaben in etwa gleicher Höhe an. Der Fonds finanziert sich aus Beiträgen der Mitgliedstaaten. Diese werden nach einem Ad-hoc-Aufbringungsschlüssel gemäß Art. 1 des internen Abkommens über die Finanzierung und Verwaltung der Hilfen der Gemeinschaft im Rahmen des vierten AKP-EWG-Abkommens von den Mitgliedstaaten aufgebracht.

Die Ausgaben für die Zusammenarbeit mit Drittländern werden in den nächsten Jahren noch an Bedeutung gewinnen, da hier die finanziellen Hilfen der Europäischen Gemeinschaft für die Staaten Osteuropas eingestellt werden. ${ }^{26}$ Für 1990 und 1991 sind Zahlungen in Höhe von 335 bzw. 310 Mio. ECU für diese Hilfen angesetzt. Die wachsende Bedeutung der Ausgaben für die $\mathrm{Zu}$ sammenarbeit mit Entwicklungsländern und Drittländern insgesamt zeigt sich daran, daß ihr Anteil an den Gesamtausgaben von etwa 2,4\% im Jahre 1988 auf 3,9\% für 1991 angestiegen ist.

25 Vgl. Rat der EG und Kommission der EG = Viertes AKP-EWG-Abkommen = $1 \mathrm{ff}$.

26 Vgl. u. a. Rat der EG =Wirtschaftshilfe für Ungarn und Polen 1989=11ff. 


\section{Ausgabenverteilung}

Tabelle 7: Die Verteilung der Finanzbeiträge auf die Mitgliedstaaten im Rahmen des siebten Europäischen Entwicklungsfonds (EEF)

\begin{tabular}{|lrr|}
\hline $\begin{array}{l}\text { Mitglied- } \\
\text { staat }\end{array}$ & $\begin{array}{r}\text { Schlüssel } \\
\text { in \% }\end{array}$ & $\begin{array}{r}\text { Gesamtausstattung } \\
\text { des 7. EEF } \\
\text { in Mio. ECU }\end{array}$ \\
\hline Belgien & 3,96 & 433,2340 \\
Dänemark & 2,08 & 227,0320 \\
Deutschland & 25,96 & 2840,4800 \\
Griechenland & 1,22 & 133,9200 \\
Spanien & 5,89 & 644,9990 \\
Frankreich & 24,37 & 2665,8920 \\
Irland & 0,55 & 60,0325 \\
Italien & 12,96 & 1417,7720 \\
Luxemburg & 0,19 & 20,7385 \\
Niederlande & 5,57 & 609,1200 \\
Portugal & 0,88 & 96,1400 \\
Großbritannien & 16,37 & 1790,6400 \\
Insgesamt & 100,00 & 10940,0000 \\
\hline
\end{tabular}

Quelle: Art. 1 des internen Abkommens über die Finanzierung und Verwaltung der Hilfen der Gemeinschaft im Rahmen des vierten AKP-EWG-Abkommens.

\section{Die Ausgaben der Europäischen Gemeinschaften und die Haushalte von Bund und Ländern}

Stellt man die Ausgaben der Gemeinschaften den nationalen Finanzen gegenüber, so kann man bei der Verteilung der Ausgabenhoheit unterschiedliche Gruppen nicht rückzahlbarer Ausgaben der Gemeinschaftsebene unterscheiden. 27

Zunächst gibt es Ausgaben der Europäischen Gemeinschaften, die früher von nationalen Gebietskörperschaften geleistet wurden und nunmehr allein von der Gemeinschaft getragen werden. Ein Beispiel dafür sind die Marktordnungsausgaben im Agrarbereich. Rein technisch werden die Agrarmarktordnungsausgaben zunächst vom Bund geleistet, der die entsprechenden Beträge jedoch in voller Höhe von den Gemeinschaften erstattet bekommt. ${ }^{28}$

27 Vgl. ähnlich Strasser $=$ Finanzen Europas $=114$.

28 Im Jahr 1991 werden dem Bund voraussichtlich Marktordnungsausgaben in Höhe von 12,9 Mrd. DM erstattet; vgl. Bundesministerium der Finanzen =Finanzbericht $1991=207$. 
Tabelle 8: Ausgaben der Strukturfonds für das Ziel 2, die im Zeitraum 1.1.1989 bis 31.12.1991 in die Bundesrepublik Deutschland fließen (in Mio. ECU)

\begin{tabular}{|c|c|c|c|c|}
\hline Gebiet & EFRE & ESF & $\begin{array}{l}\text { EFRE } \\
+ \text { ESF }\end{array}$ & $\begin{array}{c}\text { Strukturfonds- } \\
\text { mittel ergän- } \\
\text { zende natio- } \\
\text { nale Mittel }\end{array}$ \\
\hline Stadt Emden & 1,6 & 0,7 & 2,3 & 3,9 \\
\hline $\begin{array}{l}\text { Stadt Saarbrücken } \\
\text { und Landkreis } \\
\text { Saarlouis }\end{array}$ & 14,0 & 6,0 & 20,0 & 3,8 \\
\hline $\begin{array}{l}\text { Stadt Salzgitter } \\
\text { und Landkreis } \\
\text { Peine }\end{array}$ & 3,7 & 1,6 & 5,3 & 8,5 \\
\hline $\begin{array}{l}\text { Städte Pirmasens } \\
\text { und Zweibrücken } \\
\text { und Landkreis } \\
\text { Pirmasens }\end{array}$ & 8,2 & 2,0 & 10,2 & 25,9 \\
\hline Berlin & 39,0 & 21,0 & 60,0 & 70,8 \\
\hline $\begin{array}{l}\text { Nordrhein-West- } \\
\text { falen }\end{array}$ & 111,0 & 46,0 & 157,0 & 187,1 \\
\hline Bremen & 18,2 & 7,3 & 25.5 & 35,2 \\
\hline
\end{tabular}

Quelle: Kommission der EG =GFK Ziel 2 1990= 35ff.

Daneben gibt es Ausgaben, die die Gemeinschaft gemeinsam mit und in Ergänzung zu den Mitgliedstaaten vornimmt. Wichtigstes Beispiel sind hier die Ausgaben im Rahmen der Strukturfonds. Die Bundesrepublik Deutschland ${ }^{29}$ erhält dabei für das Ziel Nr. 1 (Förderung der Entwicklung und der strukturellen Anpassung der Regionen mit Entwicklungsrückstand) allerdings keine Mittel. ${ }^{30}$ Welche Mittel im Rahmen des zweiten Ziels (Umstellung der Regio-

29 Die folgenden Aussagen gelten nur für die Bundesrepublik Deutschland entsprechend dem Gebietsstand vor dem 3.10.1990.

30 Vgl. dazu Rat der EG = Aufgaben und Effizienz der Strukturfonds $1988=20$. 
nen, die von der rückläufigen industriellen Entwicklung besonders betroffen sind) in welche Regionen der Bundesrepublik Deutschland fließen und welche Mittel auf nationaler Ebene zusätzlich bereitzustellen sind, wird in Tabelle 8 aufgelistet.

Die Ausgaben für die Ziele Nr. 3 und 4 (Bekämpfung der Langzeitarbeitslosigkeit und Erleichterung der Eingliederung Jugendlicher in das Erwerbsleben) werden für das gesamte Gebiet eines Mitgliedstaates gewährt. Für den Zeitraum 1.1.1990 bis 31.12.1992 sind für entsprechende Maßnahmen auf dem Gebiet der Bundesrepublik Deutschland (entsprechend dem Gebietsstand vor dem 3.10.1990) insgesamt Mittel in Höhe von 1,4 Mrd. ECU vorgesehen. Davon gehen 573 Mio. ECU zu Lasten des Europäischen Sozialfonds, den Rest tragen nationale, regionale oder kommunale Behörden. ${ }^{31}$

Entsprechend den "Gemeinschaftlichen Förderkonzepten" zur Entwicklung der ländlichen Gebiete (Ziel Nr. 5b) unterstützen die Strukturfonds die von den Ländern vorgesehenen Entwicklungsmaßnahmen mit umfangreichen Fördermitteln. Die Gesamtkosten der Maßnahmen der Länder werden auf mehr als 1,6 Mrd. ECU veranschlagt; die Europäischen Gemeinschaften beteiligen sich mit 30 bis $50 \%$ an den einzelnen Ausgaben. ${ }^{32}$ Die Verteilung der Mittel auf die einzelnen Länder ${ }^{33}$ kann Tabelle 9 entnommen werden.

Bei Maßnahmen, die im Rahmen der Gemeinschaftsaufgaben von den Europäischen Gemeinschaften mitfinanziert werden, fließen die Mittel zunächst an den Bund, der dann die Rückvergütungen der Europäischen Gemeinschaften den Ländern anteilig erstattet. ${ }^{34}$ Es gibt jedoch auch Mittel, die direkt an die Länder fließen, wie z. B. beim Sonderprogramm für das Saarland oder beim RESIDER-Programm ${ }^{35}$, in dessen Genuß ebenfalls das Saarland, aber auch einige Gebiete in Nordrhein-Westfalen (Duisburg/Oberhausen, Bochum, Dortmund/Unna) kommen. Letzteres wird vor allem zur Sanierung von brachlie

31 Vgl. Kommission der EG =Gemeinschaftliches Förderkonzept Ziele 3 und 4 1990= $18 \mathrm{f}$.

32 Vgl. = Agrarbericht $1991=100$.

33 Die neuen Länder sind hierbei nicht enthalten.

34 Vgl. z. B. Haushaltsplan des Saarlandes für das Haushaltsjahr 1990, Einzelplan 08, S. 49.

35 Vgl. Rat der EG $=$ RESIDER-Programm $=1 \mathrm{ff}$. 
Tabelle 9: Ausgaben der Strukturfonds für das Ziel 5b, die im Zeitraum 1989 bis 1993 in die Bundesrepublik Deutschland fließen (in Mio. ECU)

\begin{tabular}{|c|c|c|c|c|}
\hline Gebiet & $\begin{array}{l}\text { EAGFL- } \\
\text { Ausrichtung }\end{array}$ & EFRE & ESF & $\begin{array}{r}\text { Struktur- } \\
\text { fonds } \\
\text { insgesamt }\end{array}$ \\
\hline Bayern & 88,805 & 108,17 & 160,233 & 257,199 \\
\hline Schleswig-Holstein & 24,736 & 10,221 & 1,517 & 36,474 \\
\hline Nordrhein-Westfalen & 4,208 & 11,798 & 3,528 & 19,534 \\
\hline Niedersachsen & 35,597 & 54,618 & 17,750 & 107,967 \\
\hline Baden-Württemberg & 10,341 & 15,254 & 3,157 & 28,752 \\
\hline Hessen & 18,757 & 16,511 & 2,729 & 37,997 \\
\hline Rheinland-Pfalz & 10,758 & 15,533 & 4,939 & 31,230 \\
\hline Saarland & 1,207 & 3,381 & 1,259 & 5,847 \\
\hline
\end{tabular}

Quelle: =Agrarbericht 1991 $=100$. 


\section{Ausgabenverteilung}

genden Industrieflächen verwendet, wobei die betroffenen Gemeinden vom Land für entsprechende Vorhaben Zuweisungen erhalten. ${ }^{36}$

Schließlich gibt es auch Ausgaben, die durch die Existenz der Gemeinschaft bedingt sind. Als Beispiele lassen sich hier die Verwaltungsausgaben der Gemeinschaft oder aber die Ausgaben für die Forschungseinrichtungen der Gemeinschaft nennen. Diese Ausgaben berühren die nationalen Haushalte nicht direkt.

36 Vgl. Haushaltsplan des Saarlandes für das Haushaltsjahr 1990, Einzelplan 08, S. 30 f. 


\section{Die Einnahmenverteilung}

\section{Das Einnahmensystem der Europäischen Gemeinschaften}

\section{a) Die Entwicklung des Einnahmensystems}

\section{aa) Das System der Umlagen und der Finanzbeiträge}

Um die gegenwärtige Ausgestaltung des Einnahmensystems der Europäischen Gemeinschaften zu verstehen, ist es sinnvoll, zunächst einen Blick auf seine Entwicklung zu werfen. Die Europäische Gemeinschaft für Kohle und Stahl verfügte von Beginn an über eigene Einnahmen, die sich im wesentlichen aus Umlagen und Anleihen zusammensetzen. Art. 49 EGKSV berechtigt die Kommission zur Erhebung von Umlagen auf die Erzeugnisse von Kohle und Stahl. Die umlagepflichtigen Erzeugnisse werden mit einem einheitlichen Satz belastet, der jährlich von der Kommission festgelegt wird. Die Gemeinschaft erhebt die Umlage ohne Mitwirkung der Finanzverwaltungen der Mitgliedstaaten unmittelbar von den Unternehmen in der Gemeinschaft. Die Unternehmen sind zu monatlichen Produktionsmeldungen an das Umlagebüro der Kommission verpflichtet und müssen die Höhe ihrer Umlageschuld selbst berechnen (Selbstveranlagungsverfahren). Die Umlage weist alle Charakteristika einer (Produktion-)Steuer auf, und ihre Kennzeichnung als erste europäische Steuer ist durchaus gerechtfertigt. ${ }^{1}$

Dagegen erfolgte die Finanzierung der Ausgaben der Haushalte der Europäischen Wirtschaftsgemeinschaft und der Europäischen Atomgemeinschaft und auch der Ausgaben des ab 1965 für beide Gemeinschaften aufgestellten Gesamthaushaltes bis zum Jahre 1974 ausschließlich über Finanzbeiträge. Diese wurden für die einzelnen Mitgliedstaaten nach einem allgemeinen Aufbringungsschlüssel, einem Schlüssel für den Sozialfonds und einem Schlüssel für die Forschungs- und Investitionsausgaben im Rahmen der Europäischen Atomgemeinschaft bestimmt.

1 Vgl. dazu Bickel $=$ Finanzausgleich $=759$ und Nittka $=$ Finanzierungssystem $=29$. 


\section{Einnahmenverteilung}

Tabelle 10: Die Aufbringungsschlüssel des Finarızsystems vor 1970 (in Prozent)

\begin{tabular}{|lrrr|}
\hline $\begin{array}{l}\text { Mitglied- } \\
\text { staat }\end{array}$ & $\begin{array}{r}\text { allgemeiner } \\
\text { Schlüssel }\end{array}$ & $\begin{array}{r}\text { Schlüssel } \\
\text { für den } \\
\text { Sozialfonds }\end{array}$ & $\begin{array}{r}\text { Schlüssel } \\
\text { für den For- } \\
\text { schungs- und } \\
\text { Investitions- } \\
\text { haushalt der }\end{array}$ \\
Belgien & & & EAG \\
Deutschland & & & 9,9 \\
Frankreich & 7,9 & 32,0 & 30,0 \\
Italien & 28,0 & 32,0 & 30,0 \\
Luxemburg & 28,0 & 20,0 & 23,0 \\
Niederlande & 28,0 & 0,2 & 0,2 \\
\hline
\end{tabular}

Quelle: Art. 200 EWGV und Art. 172 EAGV.

\section{bb) Der Eigenmittelbeschluß von 1970}

Es war jedoch von vornherein vorgesehen, nach Ablauf einer Übergangszeit die Finanzbeiträge durch ein System der eigenen Mittel zu ersetzen. ${ }^{2}$ Nach den Vorstellungen der Kommission sind Eigenmittel unmittelbar von Einzelpersonen oder Unternehmen aufzubringen und unterliegen nicht den Beschlüssen der Mitgliedstaaten. Selbst wenn die eigenen Mittel von den Mitgliedstaaten eingezogen werden, geschieht dies im Namen der Gemeinschaft. "Die Mittel sind nicht Teil der Einnahmen der Mitgliedstaaten, und brauchen nicht im Haushaltsplan der Mitgliedstaaten ausgewiesen und durch die mitgliedstaatlichen Parlamente genehmigt zu werden."3

Am 21.4.1970 wurde der Beschluß für ein System der Eigenmittel ${ }^{4}$ gefaßt, der der Gemeinschaft als eigentliche eigene Mittel Zölle und Agrarabschöpfungen und für die Restfinanzierung des Haushaltes Mehrwertsteuer-Eigenmittel zuwies.

2 Vgl. Art. 201 EWGV.

3 Kommission der EG $=$ Finanzierung des Gemeinschaftshaushalts $=17$.

4 Vgl. Rat der EG = Eigenmittelbeschluß 1970= 19ff. 
Agrarabschöpfungen und Zölle werden auch als "traditionelle"5 oder "originäre"6 Eigenmittel bezeichnet. Nach den oben genannten Kriterien stellen sie echte eigene Mittel dar. Sie beruhen unmittelbar auf der Existenz eines einheitlichen Zollraumes und ihre Zuordnung an die Mitgliedstaaten ist rechtlich oder praktisch nicht möglich. ${ }^{7}$ Ihr gesamtes Aufkommen geht direkt in das Eigentum der Gemeinschaft über. Die Entscheidungen über die Höhe dieser Abgaben werden von den Organen der Gemeinschaft gefaßt. ${ }^{8}$

Bei den Agrarabschöpfungen lassen sich die Abschöpfungen im eigentlichen Sinne sowie Zucker- und Isoglukoseabgaben unterscheiden. Durch die eigentlichen Agrarabschöpfungen sollen die im Rahmen der Gemeinsamen Agrarmarktordnungen festgelegten Richtpreise nach außen gesichert werden. Entsprechenden Agrarprodukten aus Drittländern, die auf dem Weltmarkt zu niedrigeren Preisen angeboten werden, wird beim Import die Differenz zwischen Weltmarktpreis und Richtpreis der Gemeinschaft als Importausgleichsabgabe auferlegt. Die Zuckerabgaben zahlen die Unternehmen des Zuckersektors. Auf die von der Gemeinschaft festgesetzten Produktionsquoten, für die es auf dem Zuckermarkt Preisgarantien gibt, werden Abgaben in Höhe eines bestimmten Prozentsatzes des garantierten Interventionspreises erhoben (Produktionsabgabe). Daneben wird eine Lagerkostenabgabe erhoben, mit der die Kosten der Lagerhaltung abgedeckt werden sollen und die von den Zuckerfabriken beim Verkauf von Zucker erhoben wird. Die Abgaben auf die Isoglukoseproduktion haben dieselbe Funktion wie die Zuckerabgaben. ${ }^{9}$

Obwohl Zölle grundsätzlich zu den "originären" Eigenmitteln gehören, wurden zunächst die Zölle auf Erzeugnisse, die unter den Vertrag zur Gründung der Europäischen Gemeinschaft für Kohle und Stahl fallen, nicht von der Gemeinschaft, sondern von den Mitgliedstaaten erhoben.

Von den Agrarabschöpfungen und den Zöllen wurden bis zur Neuregelung 1988 den Mitgliedstaaten 10\% der abzuführenden Beträge für die Erhebungskosten erstattet.

5 Vgl. Kommission der EG =Öffentliche Finanzen $=57$.

6 Vgl. Messal $=$ EG-Finanzierung $=9$.

7 Vgl. Kommission der EG =Öffentliche Finanzen $=57$.

8 Vgl. Kommission der EG =Finanzierung des Gemeinschaftshaushalts $=17$.

9 Vgl. Kommission der EG =Öffentliche Finanzen $=58$, vgl. dazu auch D. III. 2. b). 


\section{Einnahmenverteilung}

Im Beschluß vom 21.4.1970 waren neben den "originären" Eigenmitteln auch Mehrwertsteuer-Eigenmittel vorgesehen, die den Haushalt durch Anwendung eines einheitlichen Satzes von maximal 1\% auf eine für alle Mitgliedstaaten nach Gemeinschaftsvorschriften einheitlich bestimmte Mehrwertsteuer-Bemessungsgrundlage ausgleichen sollte. Die erforderliche Harmonisierung der nationalen Bemessungsgrundlagen konnte allerdings nur mit großen Verzögerungen erreicht werden, so daß die Mehrwertsteuer-Einnahmen von sämtlichen Mitgliedstaaten erst mit Beginn des Jahres 1980 abgeführt wurden. ${ }^{10}$

Die Mehrwertsteuer-Bemessungsgrundlage eines Mitgliedstaates besteht aus der Summe aller harmonisierten steuerpflichtigen Umsätze (auf der Stufe des Endverbrauchs). Für ihre Ermittlung sind zwei Methoden zulässig: die Einnahmenmethode und die Methode der Festlegung anhand der Steuererklärungen. ${ }^{11}$

Bei der inzwischen ausschließlich verwendeten Einnahmenmethode werden die nationalen Mehrwertsteuer-Einnahmen zunächst um diejenigen Einnahmen korrigiert, die auf Umsätzen beruhen, welche nach den gemeinschaftlichen Vorschriften nicht Teil der harmonisierten Mehrwertsteuer-Bemessungsgrundlage sind, wie etwa Umsätze, die auf der Tätigkeit der öffentlichen Rundfunkund Fernsehanstalten beruhen. 12 Auf diese Nettoeinnahmen wird ein geschätzter gewogener mittlerer Satz angewandt, d. h. es wird ein auf die verschiedenen Kategorien steuerbarer Gegenstände anwendbarer Durchschnittssatz geschätzt. Gäbe es nur einen einzigen Mehrwertsteuersatz, so könnte man die Nettoeinnahmen einfach durch diesen dividieren, um die Bemessungsgrundlage zu erhalten. Da jeder Mitgliedstaat jedoch zwei oder mehr Mehrwertsteuersätze anwendet, muß aus diesen Sätzen ein Durchschnitt gebildet werden.

Die Gewichte der Sätze sind dabei die den jeweiligen Mehrwertsteuersätzen zurechenbaren Anteile der Umsätze am Gesamtumsatz des Mitgliedstaates. Die entsprechenden Daten aus den Volkswirtschaftlichen Gesamtrechnungen stehen

$10 \mathrm{Vgl}$. Kommission der EG =Öffentliche Finanzen $=57$.

11 Der Europäische Rechnungshof ist der Auffassung, daß die beiden Berechnungsmethoden nicht ergebnisneutral sind, da das Einnahmenverfahren zu nicht unerheblich geringeren Mehrwertsteuerabführungen führt. Vgl. Europäischer Rechnungshof =Jahresbericht $1985=31$.

12 Vgl. zu diesen Ausnahmen Rat der EG = Sechste Richtlinie zur Umsatzsteuerharmonisierung = Anhang $\mathrm{E}$ in Verbindung mit Rat der EG = Achtzehnte Richtlinie zur Umsatzsteuerharmonisierung $=$ Art. 1 Nr. 4 . 
nur mit zeitlicher Verzögerung zur Verfügung, daher wird der gewogene Satz eines bestimmten Jahres aus Daten errechnet, die zwei, manchmal sogar drei Jahre zurückliegen. 13

Schließlich werden zu der so errechneten Bemessungsgrundlage noch diejenigen Umsätze hinzugerechnet, die eigentlich nach den Gemeinschaftsvorschriften Bestandteil der harmonisierten Mehrwertsteuer-Bemessungsgrundlage sind, aber aufgrund von Ausnahmeregelungen nicht der nationalen Besteuerung unterliegen. Ein wichtiges Beispiel hierfür ist die Steuerbefreiung der Umsätze von Gold, das nicht für industrielle Zwecke bestimmt ist. ${ }^{14}$

In Anlehnung an Messal ${ }^{15}$ läßt sich die Berechnung der Mehrwertsteuer-Bemessungsgrundlage (X) eines Mitgliedstaates (i) in folgender Formel zusammenfassen:

$$
X_{i}^{t}=\left(e_{i}^{t}-C_{i, 1}{ }^{t}\right): s_{i}^{t-2}+C_{i, 2}{ }^{t}
$$

ei = die nationalen Mehrwertsteuer-Einnahmen

$\mathrm{C}_{\mathrm{i}, 1}{ }^{\mathrm{t}}=$ erstes Korrektiv (Einnahmen, die auf Umsätzen beruhen, die nach den Harmonisierungsvorschriften nicht Teil der Bemessungsgrundlage sind)

$\mathrm{s}_{\mathrm{i}} \quad=$ der gewogene mittlere Mehrwertsteuer-Satz

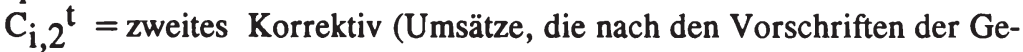
meinschaft Bestandteil der harmonisierten Bemessungsgrundlage sind, aber aufgrund von Ausnahmeregelungen nicht der nationalen Besteuerung unterliegen)

t $\quad=$ Zeitindex

13 Vgl. Messal =EG-Finanzierung $=15$.

14 Vgl. Rat der EG = Sechste Richtlinie zur Umsatzsteuerharmonisierung = Anhang F in Verbindung mit Rat der EG = Achtzehnte Richtlinie zur Umsatzsteuerharmonisierung = Art. 1 Nr. 4.

15 Vgl. Messal $=$ EG-Finanzierung $=16$. 


\section{Einnahmenverteilung}

Entsprechend dem Eigenmittelbeschluß von 1970 dienten die MehrwertsteuerEigenmittel der Restfinanzierung des Haushaltes; der Teil der Ausgaben, der nicht durch originäre Eigenmittel und sonstige Einnahmen ${ }^{16}$ gedeckt war, wurde durch die Mehrwertsteuer-Eigenmittel aufgebracht. Dementsprechend wurde im Haushaltsverfahren ein Satz festgelegt, der auf die MehrwertsteuerBemessungsgrundlage der Mitgliedstaaten angewandt wurde. Dieser Satz war auf $1 \%$ für jeden Mitgliedstaat begrenzt.

\section{cc) Der Eigenmittelbeschluß von 1985}

Durch den Eigenmittelbeschluß vom 7.5.1985 wurde der maximale Abführungssatz der Mehrwertsteuer auf 1,4\% der Bemessungsgrundlage angehoben. ${ }^{17}$ Im selben Jahr wurde ein Korrekturmechanismus für Großbritannien eingeführt, der für ein bestimmtes Jahr stets im Haushalt des Folgejahres über die Mehrwertsteuer-Eigenmittel abzuwickeln war. Großbritannien erhielt einen Ausgleichsanspruch in Höhe von $66 \%$ der Differenz zwischen seinem Mehrwertsteuer-Eigenmittelanteil und seinem Anteil ${ }^{\mathbf{1 8}}$ an den umlegbaren Ausgaben ${ }^{19}$ zuerkannt. Dieser Anspruch war von den anderen Mitgliedstaaten zu finanzieren. Für die Bundesrepublik Deutschland (neben Großbritannien der einzige Nettozahler) war allerdings eine Sonderregelung getroffen worden: Sie hatte nur zwei Drittel ihres Anteils aufzubringen. Dies führte dazu, daß sich der Satz auf die Mehrwertsteuer-Bemessungsgrundlage für Großbritannien verminderte, dagegen für die übrigen Mitgliedstaaten erhöhte. Da außerdem die Sonderregelung für die Bundesrepublik Deutschland beachtet werden muß,

16 Zu den sonstigen Einnahmen zählen vor allem Überschüsse aus vorhergehenden Haushaltsjahren sowie Steuem und Abzüge auf Löhne und Gehälter von bei den Europäischen Gemeinschaften Beschäftigten. Dabei wird je nach Einkommenshöhe ein Steuersatz zwischen $8 \%$ und $45 \%$ auf die monatlichen Bezüge der bei den Europäischen Gemeinschaften Beschäftigten erhoben. Vgl. dazu Europäisches Parlament =Haushaltsplan $1991=261$.

17 Vgl. Rat der EG =Eigenmittelbeschluß 1985=.

18 Da die Mehrwertsteuer-Eigenmittel geringer sind als der Gesamtbetrag der Ausgaben, muß auf die Anteile zurückgegriffen werden. Vom Mehrwertsteuer-Eigenmittelanteil wird der Rückflußanteil abgezogen. Diese Differenz (ein Prozentpunktesatz) wird dann multipliziert mit dem Gesamtvolumen der Ausgaben und so erhält man einen absoluten Betrag (in ECU): einen fiktiven Nettosaldo als Basis für den Ausgleichsanspruch. Vgl. 'Messal =EG-Finanzierung $=28$ und Augstein =Finanzwirtschaft der $\mathrm{EG}=329$.

19 Nicht alle Ausgaben können einzelnen Mitgliedstaaten zugerechnet werden; so fließen etwa die Ausgaben für die Entwicklungshilfe überhaupt nicht in die Mitgliedstaaten zurück. Daher können nur die zurechenbaren Ausgaben berücksichtigt werden. 
ergeben sich für die Mehrwertsteuer-Eigenmittelsätze der einzelnen Mitgliedstaaten unterschiedliche Höhen. ${ }^{20}$

Kein Mitgliedstaat durfte insgesamt mehr als $1,4 \%$ seiner MehrwertsteuerBemesungsgrundlage abführen. Daher stieß die Gemeinschaft an diese Grenze von $1,4 \%$, als diejenigen Mitgliedstaaten, die sich an der Finanzierung des Ausgleichs zu beteiligen hatten, einschließlich ihrer Beiträge zu diesem Ausgleich einen Satz von $1,4 \%$ erreicht hatten. Die Obergrenze für den anzuwendenden Satz wurde für die einzelnen Mitgliedstaaten und nicht für den Gemeinschaftsdurchschnitt definiert. Folglich blockierte die Abwicklung des Korrekturmechanismus für Großbritannien Finanzmittel, die damit nicht zur Ausgabenfinanzierung zur Verfügung standen. ${ }^{21}$

\section{b) Das derzeitige Einnahmensystem}

aa) Änderungen bei den "originären" Eigenmitteln durch den Eigenmittelbeschluß von 1988

Die vorerst letzte Änderung des Einnahmensystems erfolgte durch den Eigenmittelbeschluß von 1988. ${ }^{22}$ Nach diesem Beschluß werden außer den bisherigen "originären" Eigenmitteln auch die Zölle auf die unter den Vertrag zur Gründung der Europäischen Gemeinschaft für Kohle und Stahl fallenden Erzeugnisse nicht mehr von den Mitgliedstaaten, sondern von der Gemeinschaft erhoben. Die Abwicklung der Pauschalabgeltung der Erhebungskosten für die "originären" Mittel wurde insofern geändert, als sie nicht mehr als Ausgaben, sondern als negative Einnahmen behandelt und unmittelbar von den Einnahmen abgezogen werden. Dies entspricht allerdings nicht der ursprünglichen Vorstellung der Kommission, die die Erstattung abschaffen wollte, "da sie keineswegs leicht bezifferbaren und den einzelnen Mitgliedstaaten zurechenbaren Ist-Ausgaben entspricht" 23 .

20 Im Jahr 1986 betrug der Satz für Großbritannien 0,68\%, für die Bundesrepublik Deutschland 1,34\% und für die übrigen Mitgliedstaaten 1,4\%.

$21 \mathrm{Vgl}$. Messal =EG-Finanzierung = 20f. und 31f.

22 Vgl. Rat der EG =System der Eigenmittel $1988=24 \mathrm{ff}$.

23 Kommission der EG = Öffentliche Finanzen $=58$. 


\section{Einnahmenverteilung}

\section{bb) Mehrwertsteuer-Eigenmittel und Bruttosozialprodukt-Eigenmittel}

Bei den Mehrwertsteuer-Eigenmitteln wurde beschlossen, die Bemessungsgrundlage bei der Berechnung der abzuführenden Mehrwertsteuer-Eigenmittel nur noch bis zu einer Höhe von $55 \%$ des Bruttosozialproduktes des jeweiligen Mitgliedstaates heranzuziehen. ${ }^{24}$ Das bedeutet, daß die Mehrwertsteuer-Bemessungsgrundlage $\left(\mathrm{X}_{\mathrm{i}}\right)$ eines jeden Mitgliedstaates mit seinem Bruttosozialprodukt verglichen wird. Bei den Mitgliedstaaten, deren Mehrwertsteuer-Bemessungsgrundlage 55\% des Bruttosozialprodukts überschreitet, wird der Mehrwertsteuer-Eigenmittelsatz nicht mehr auf die gesamte Mehrwertsteuer-Bemessungsgrundlage angewandt, sondern nur noch auf den Teil, der 55\% des Bruttosozialprodukts entspricht (Kappung der Mehrwertsteuer-Bemessungsgrundlage). Der Mehrwertsteuer-Eigenmittelhöchstsatz blieb für jeden Mitgliedstaat bei 1,4\%. Die Funktion der Restfinanzierung des Haushaltes wird nun allerdings nicht mehr von den Mehrwertsteuer-Eigenmitteln, sondern von einer vierten Eigenmittelart übernommen. Wenn Zölle, Agrarabschöpfungen, Mehrwertsteuer-Eigenmittel und sonstige Einnahmen die Ausgaben nicht decken, wird der Rest durch die Anwendung eines flexiblen, aber für alle Mitgliedstaaten (grundsätzlich) einheitlichen Satzes auf das Bruttosozialprodukt zu Marktpreisen aufgebracht (Bruttosozialprodukt-Eigenmittel).

\section{cc) Einführung einer Obergrenze für die Einnahmen insgesamt}

Neu ist auch, daß eine Obergrenze für alle Eigenmittelarten zusammen in Höhe von $1,2 \%$ des gesamten Bruttosozialproduktes der Gemeinschaft eingeführt wurde. Im Zeitraum von 1988 bis 1992 darf der Gesamtbetrag der Eigenmittel zusätzlich folgende Grenzen nicht überschreiten:

1988: $1,15 \%$

1989: $1,17 \%$

1990: $1,18 \%$

1991: $1,19 \%$

1992: $1,20 \% .^{25}$

24 Vgl. Rat der EG $=$ System der Eigenmittel $1988=25$.

25 Vgl. Rat der EG $=$ System der Eigenmittel $1988=26$. 
Durch diese neue Obergrenze und die vierte Eigenmittelart wurde der Finanzrahmen der Europäischen Gemeinschaften deutlich erweitert.

\section{dd) Die Anpassung der Ausgleichsregelung für Großbritannien ${ }^{26}$}

Die Finanzierung der Ausgleichsregelung für Großbritannien wurde fortgesetzt. Allerdings sind die Auswirkungen durch die Änderung des Finanzierungssystems zu berücksichtigen. Der Ausgleichsanspruch Großbritanniens errechnet sich aus dem Anspruch, wie er seit 1985 bestand, ${ }^{27}$ abzüglich der finanziellen Auswirkungen der Änderung des Finanzierungssystems.

Diese errechnen sich dadurch, daß der Anteil Großbritanniens an den Mehrwertsteuer-Eigenmitteln, der sich ohne die Berücksichtigung der Kappung der Bemessungsgrundlage - also wie 1985 - ergibt, multipliziert wird mit der Summe aus den Mehrwertsteuer-Einnahmen mit Berücksichtigung der Kappung und aus den Bruttosozialprodukt-Einnahmen. Davon werden die Mehrwertsteuer-Eigenmittel, die Großbritannien unter Berücksichtigung der Kappung leistet, und die von Großbritannien gezahlten Bruttosozialprodukt-Beiträge abgezogen. Der so berechnete neue Ausgleichsbetrag wird Referenzbetrag genannt.

Zur Finanzierung des Ausgleichs werden die übrigen Mitgliedstaaten gemäß ihrem Anteil am Bruttosozialprodukt herangezogen, wobei die Bundesrepublik Deutschland nur zwei Drittel ihres Anteils trägt. Die Abwicklung der Finanzierung des Korrekturmechanismus erfolgt jedoch weiterhin über die Mehrwertsteuer-Eigenmittel. Im Extremfall kommt es zu zwölf verschiedenen Mehrwertsteuer-Abführungssätzen. Allerdings gilt auch hier die Obergrenze von $1,4 \%$ für jeden Mitgliedstaat. Stößt ein Mitgliedstaat an diese Grenze, wird die Finanzierung des Ausgleichs auch auf die Bruttosozialprodukt-Eigenmittel ausgeweitet. Im folgenden seien diese Vorgänge am Beispiel des Haushaltsjahres 1991 veranschaulicht.

26 Vgl. dazu Messal $=$ EG-Finanzierung $=74 \mathrm{ff}$.

27 Vgl. C. V. 1.a) cc). 


\section{Einnahmenverteilung}

Tabelle 11: Zur Berechnung des Referenzbetrages

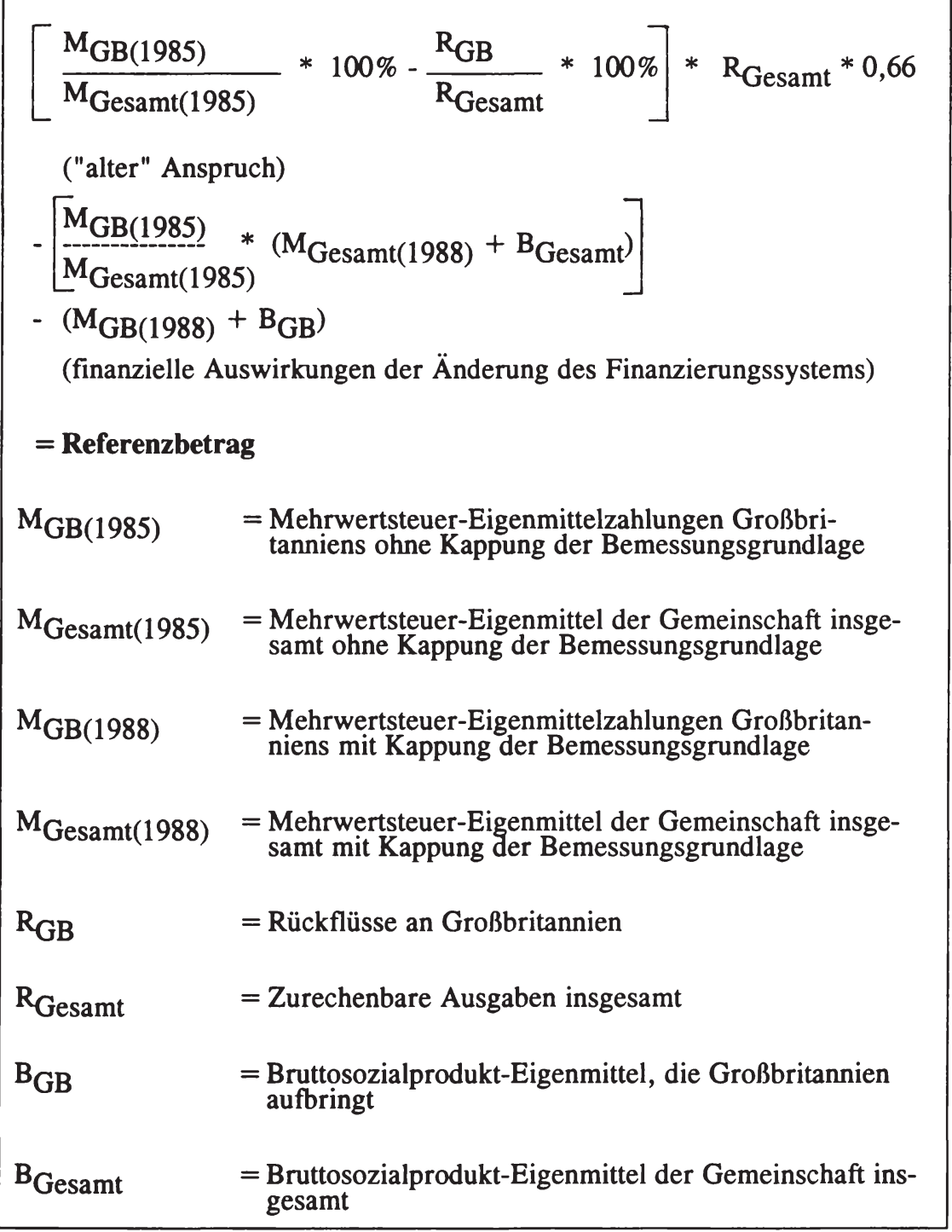


ee) Die Berechnung der Anteile an den Mehrwertsteuer-Eigenmitteln und den Bruttosozialprodukt-Eigenmitteln der Mitgliedstaaten für das Haushaltsjahr $1991^{28}$

Vom Gesamtbetrag der Ausgaben in Höhe von 55.556,1 Mio. ECU werden die Zölle und Agrarabschöpfungen sowie die sonstigen Einnahmen in Höhe von 16.562,1 Mio. ECU abgezogen. Von den verbleibenden 38.994,0 Mio. ECU werden 30.666,2 Mio. ECU über die Mehrwertsteuer-Eigenmittel finanziert. Dieser Betrag ergibt sich, indem auf die Mehrwertsteuer-Bemessungsgrundlage der Mitgliedstaaten insgesamt ein Satz von 1,4\% angewandt und davon der Bruttobetrag des Referenzbetrages für den Ausgleich zugunsten Großbritanniens abgezogen wird. "Der Bruttobetrag ist der Betrag der Ausgleichszahlung, der wegen der Nichtbeteiligung des Vereinigten Königreichs an der Finanzierung seines eigenen Anteils und der Senkung des Anteils der Bundesrepublik Deutschland um ein Drittel entsprechend angepaßt wird."29

Da die Mehrwertsteuer-Bemessungsgrundlage aller Mitgliedstaaten ${ }^{30}$ zusammen 25.258,5 Mio. ECU beträgt, läge der (hypothetische) einheitliche Satz auf die Mehrwertsteuer-Bemessungsgrundlage bei 30.666,2 : 25.258,5 = $1,2141 \%$. Dieser Satz kommt jedoch wegen der Korrektur für Großbritannien in Höhe von 3.344 Mio. ECU nicht zum Tragen. Die Verteilung der Finanzierung der Korrektur wird in Tabelle 12 aufgezeigt. Die Sonderregelung für die Bundesrepublik Deutschland bewirkt dabei, daß der deutsche Anteil an der Finanzierung des Korrekturmechanismus kleiner ist als der Frankreichs und Italiens.

Tabelle 13 zeigt die Berücksichtigung des Korrekturmechanismus für Großbritannien bei der Ermittlung der endgültigen Mehrwertsteuer-Eigenmittelsätze. Durch die 1,4\%-Obergrenze bei den Mehrwertsteuer-Eigenmitteln muß ein Teil der Korrektur für Großbritannien über die Bruttosozialprodukt-Eigenmittel abgewickelt werden.

28 Vgl. dazu Europäisches Parlament $=$ Haushaltsplan $1991=97 \mathrm{ff}$.

29 Rat der EG =System der Eigenmittel 1988= 26.

30 Unter Berücksichtigung der Kappung bei $55 \%$ des Bruttosozialprodukts. 
Tabelle 12: Die Finanzierung des Korrekturmechanismus für Großbritannien im Jahre 1991

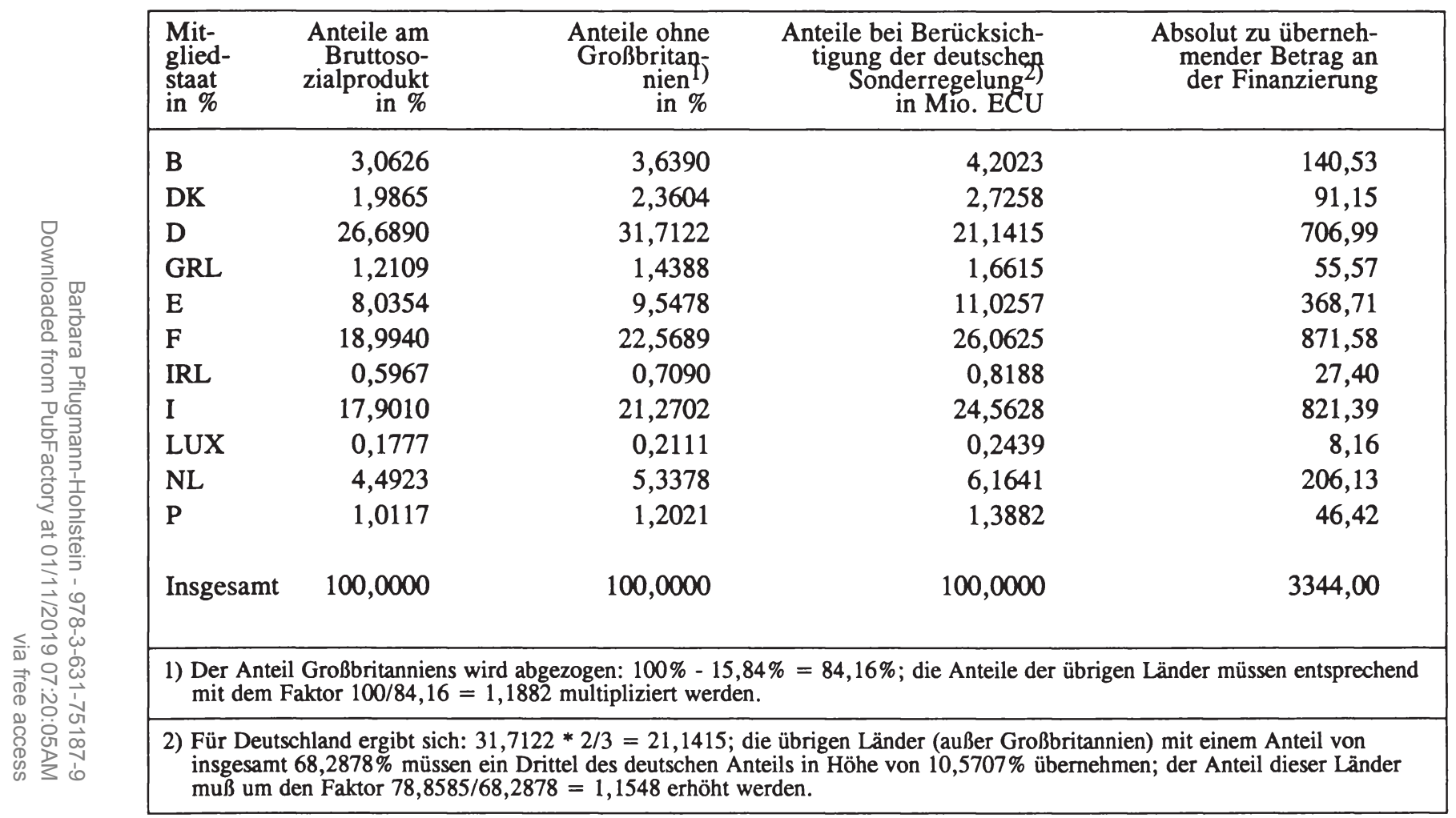


Tabelle 13: Ermittlung der Mehrwertsteuer(MWSt)-Eigenmittel im Jahre 1991 (in Mio. ECU)

\begin{tabular}{|c|c|c|c|c|c|c|c|c|}
\hline $\begin{array}{l}\text { Mit- } \\
\text { glied- } \\
\text { staat }\end{array}$ & $\begin{array}{l}1 \% \text { der } \\
\text { MWSt-Be- } \\
\text { messungs- } \\
\text { grundlage }\end{array}$ & $\begin{array}{r}\text { MWSt-Ei- } \\
\text { genmittel } \\
\text { bei ein- } \\
\text { heitl. } \\
\text { Satz1) }\end{array}$ & $\begin{array}{r}\text { Korrektur } \\
\text { für } \mathrm{GB}^{2} \text { ) }\end{array}$ & $\begin{array}{l}\text { Insgesamt } \\
(\mathrm{Sp} .3+4)\end{array}$ & $\begin{array}{r}\text { Grenze in } \\
\text { Höhe von } \\
1,4 \%\end{array}$ & $\begin{array}{r}\text { Über BSP- } \\
\text { Eigenmittel } \\
\text { abzuwickeln }\end{array}$ & $\begin{array}{r}\text { Abzuführen- } \\
\text { de MWSt- } \\
\text { Mittel }\end{array}$ & $\begin{array}{r}\text { Tatsäch- } \\
\text { licher } \\
\text { Satz } \\
\text { in \% }\end{array}$ \\
\hline $\begin{array}{l}\text { B } \\
\text { DK } \\
\text { D } \\
\text { GRL } \\
\text { E } \\
\text { F } \\
\text { IRL } \\
\text { I } \\
\text { LUX } \\
\text { NL } \\
\text { P } \\
\text { UK }\end{array}$ & $\begin{array}{r}730,4 \\
434,9 \\
6637,7 \\
341,0 \\
2150,0 \\
5120,0 \\
168,0^{4)} \\
3725,0 \\
50,14) \\
1156,0 \\
284,94) \\
\left.4460,5^{4}\right)\end{array}$ & $\begin{array}{r}886,8 \\
528,0 \\
8058,8 \\
414,0 \\
2610,3 \\
6216,2 \\
204,0 \\
4522,5 \\
60,7 \\
1403,5 \\
345,9 \\
5415,5\end{array}$ & $\begin{array}{r}140,5 \\
91,2 \\
707,0 \\
55,6 \\
368,7 \\
871,5 \\
27,4 \\
821,4 \\
8,2 \\
206,1 \\
46,4 \\
-3344,0\end{array}$ & $\begin{array}{r}1027,3 \\
619,2 \\
8765,8 \\
469,6 \\
2979,0 \\
7087,7 \\
231,4 \\
5343,9 \\
68,9 \\
1609,6 \\
392,3 \\
2071,5\end{array}$ & $\begin{array}{r}1022,6 \\
608,9 \\
9292,8 \\
477,4 \\
3010,0 \\
7168,0 \\
235,2 \\
5215,0 \\
70,1 \\
1618,4 \\
398,9 \\
6244,7\end{array}$ & $\begin{array}{r}4,7 \\
10,3 \\
- \\
- \\
- \\
- \\
- \\
128,9 \\
- \\
- \\
- \\
-\end{array}$ & $\begin{array}{r}1022,6 \\
608,9 \\
8765,8 \\
469,6 \\
2979,0 \\
7087,7 \\
231,4 \\
5215,0 \\
68,9 \\
1609,6 \\
392,3 \\
2071,5\end{array}$ & $\begin{array}{l}1,400 \\
1,400 \\
1,321 \\
1,377 \\
1,386 \\
1,384 \\
1,377 \\
1,400 \\
1,377 \\
1,392 \\
1,377 \\
0,464\end{array}$ \\
\hline Summe ${ }^{3)}$ & 25258,5 & 30666,2 & 0,0 & 30666,2 & 35361,9 & 143,9 & 28431,7 & - \\
\hline \multicolumn{9}{|c|}{$\begin{array}{l}\text { 1) Hypothetischer einheitlicher Satz von } 1,2141 \% \text {. } \\
\text { 2) Vgl. Tabelle } 12 ; \text { hier gerundete Werte. } \\
\text { 3) Kleine Unstimmigkeiten sind durch Rundungen bedingt. } \\
\text { 4) In diesen Ländern wurde die Bemessungsgrundlage gekappt, d. h. die Mehrwertsteuer-Bemessungsgrundlage wird nur bis } \\
\text { zu einer Höhe von } 55 \% \text { des Bruttosozialprodukts berücksichtigt. }\end{array}$} \\
\hline
\end{tabular}


Tabelle 14: Die Bruttosozialprodukt-Eigenmittel 1991 (in Mio. ECU)

\begin{tabular}{|lrrrr|}
\hline $\begin{array}{l}\text { Mit- } \\
\text { glied- } \\
\text { staat }\end{array}$ & 1\% des BSP & $\begin{array}{r}\text { BSP-Einnahmen } \\
\text { zum einheitlichen } \\
\text { Satz von 0,1627\% }\end{array}$ & $\begin{array}{r}\text { Beträge der nicht } \\
\text { durch MWSt-Anteile } \\
\text { gedeckten Korrektur }\end{array}$ & $\begin{array}{r}\text { Insgesamt } \\
\text { abzunuhren- } \\
\text { der Betrag }\end{array}$ \\
\hline B & 1568,0 & 255,1 & 4,7 & 259,8 \\
DK & 1017,1 & 165,4 & 10,3 & 175,7 \\
D & 13664,5 & 2222,7 & - & 2222,7 \\
E & 4114,0 & 669,2 & - & 669,2 \\
F & 9725,0 & 1581,9 & - & 1581,9 \\
GRL & 620,0 & 100,8 & - & 100,8 \\
IRL & 305,5 & 49,7 & 128,9 & 49,7 \\
I & 9165,0 & 1490,8 & - & 1619,7 \\
LUX & 91,0 & 14,8 & - & 14,8 \\
NL & 2300,0 & 374,1 & - & 84,1 \\
P & 518,0 & 84,3 & 143,9 & 1319,2 \\
UK & 8110,0 & 1319,2 & & 8471,8 \\
Summe & 51198,1 & 8327,9 & & \\
\hline BSP = Bruttosozialprodukt & & & - \\
MWSt = Mehrwertsteuer & & & & \\
\hline
\end{tabular}


Zur Berechnung der Bruttosozialprodukt-Eigenmittel muß zunächst der nach Abzug von "originären" Einnahmen, sonstigen Einnahmen und Mehrwertsteuer-Eigenmittel verbleibende Restbetrag ermittelt werden. Den Bruttosozialprodukt-Eigenmittelsatz erhält man, indem der Restbetrag durch die gesamte Bruttosozialprodukt-Bemessungsgrundlage geteilt wird. Im Jahr 1991 betrug dieser Satz daher 8.327,9/5.119.810,0 *100\% =0,1627\%. Zu den nach diesem Satz berechneten Beträgen muß jedoch noch bei den Mitgliedstaaten, die an die 1,4\%-Obergrenze bei den Mehrwertsteuer-Eigenmittel gestoßen sind, der entsprechende Restbetrag hinzuaddiert werden. Dies ist in Tabelle 14 dargestellt.

\section{c) Zur Bedeutung der einzelnen Einnahmenarten}

Die Bedeutung der einzelnen Einnahmen der Gemeinschaft läßt sich aus der Tabelle 15 ablesen. Das Gewicht der "originären" Eigenmittel hat im Laufe der Zeit deutlich abgenommen. Stellten sie im Jahre 1975 noch einen Anteil von über $50 \%$ der Gesamteinnahmen, so fiel ihr Anteil auf ca. $30 \% \mathrm{im} \mathrm{Jahr}$ 1989. Während bei den Zöllen trotz fallendem relativen Anteil in der Regel noch absolut steigende Einnahmen zu verzeichnen waren, sanken die Agrarabschöpfungen nicht nur relativ, sondern teilweise in absoluten Beträgen.

Bis zur vollständigen Einführung der Mehrwertsteuer-Eigenmittel im Jahre 1980 war der Anteil der Finanzbeiträge noch recht beachtlich. Auch nach der Verwirklichung des Eigenmittelbeschlusses von 1970 wurden die Finanzbeiträge nicht völlig abgeschafft, sondern wurden für die neu beigetretenen Mitgliedstaaten während einer Übergangsfrist als deren Beitrag zum Gemeinschaftshaushalt beibehalten. Seit das Mehrwertsteuer-Eigenmittelsystem eingerichtet wurde, hat der Anteil dieser Mittel (mit einigen Schwankungen) deutlich zugenommen. Die Mehrwertsteuer-Einnahmen erhöhen sich entsprechend der durch das wirtschaftliche Wachstum bedingten Zunahme des Endverbrauches im Laufe der Zeit sozusagen "automatisch". Dies ist bei den "originären" Einnahmen nicht in gleichem Maße der Fall. Durch die Einführung der Bruttosozialprodukt-Eigenmittel in Verbindung mit der 1,4\%-Grenze wird die Steigerung des Mehrwertsteuer-Eigenmittelanteils allerdings begrenzt. Der Anteil der Bruttosozialprodukt-Eigenmittel hängt seinerseits weitgehend vom Volumen des Gemeinschaftshaushaltes ab, da diese Mittel der Restfinanzierung dienen. 
Tabelle 15: Die Einnahmen des Gemeinschaftshaushalts nach Einnahmearten in \% der Gesamteinnahmen in den Jahren 1978 - 1990

\begin{tabular}{|c|c|c|c|c|c|c|c|c|c|c|c|c|c|}
\hline & 1978 & 1979 & 1980 & 1981 & 1982 & 1983 & 1984 & 1985 & 1986 & 1987 & 1988 & 1989 & 1990 \\
\hline $\begin{array}{l}\text { Zölle } \\
\text { Agrarab- }\end{array}$ & 36,0 & 35,6 & 37,1 & 34,6 & 31,8 & 28,2 & 30,6 & 29,6 & 24,3 & 25,0 & 24,7 & 24,7 & 24,6 \\
\hline $\begin{array}{l}\text { schöpfungen } \\
\text { Zuckerabgaben } \\
\text { Erhebungs- }\end{array}$ & $\begin{array}{r}15,4 \\
3,4\end{array}$ & $\begin{array}{r}11,5 \\
3,2\end{array}$ & $\begin{array}{l}9,7 \\
2,9\end{array}$ & $\begin{array}{l}6,9 \\
2,6\end{array}$ & $\begin{array}{l}7,1 \\
3,3\end{array}$ & $\begin{array}{l}5,4 \\
3,8\end{array}$ & $\begin{array}{l}4,8 \\
4,5\end{array}$ & $\begin{array}{l}4,0 \\
3,8\end{array}$ & $\begin{array}{l}3,5 \\
3,3\end{array}$ & $\begin{array}{l}4,5 \\
4,1\end{array}$ & $\begin{array}{l}3,6 \\
3,3\end{array}$ & $\begin{array}{l}3,2 \\
2,9\end{array}$ & $\begin{array}{l}2,5 \\
2,0\end{array}$ \\
\hline $\begin{array}{l}\text { kosten 1) } \\
\text { MWST-Eigen- }\end{array}$ & - & - & - & - & - & - & - & - & - & - & $-3,2$ & $-3,1$ & $-2,7$ \\
\hline & - & 32,4 & 46,3 & 52,8 & 55,4 & 54,6 & 54,7 & 54,5 & 67,1 & 64,6 & 58,1 & 58,5 & 59,0 \\
\hline $\begin{array}{l}\text { mittel } \\
\text { Eigenmittel }\end{array}$ & - & - & - & - & - & - & - & - & - & - & $10,7^{3)}$ & 8,7 & 0,2 \\
\hline insgesamt & 54,8 & 82,7 & 96,0 & 96,9 & 97,9 & 92,0 & 94,6 & 91,9 & 98,2 & 98,2 & 97,2 & 94,9 & 85,4 \\
\hline $\begin{array}{l}\text { Finanzbeiträge } \\
\text { verfügbare }\end{array}$ & 43,8 & 15,8 & - & 0,8 & 0,9 & 0,9 & 0,9 & 0,9 & 0,6 & 0,6 & 0,5 & - & 0,2 \\
\hline Überschüsse & - & 0,2 & 2,9 & 1,3 & - & 6,0 & 1,2 & - & 0,2 & - & 1,2 & 4,5 & 13,2 \\
\hline $\begin{array}{l}\text { Vorschüsse } \\
\text { verschiedene }\end{array}$ & - & - & - & - & - & - & 2,3 & 5,9 & 0,2 & - & - & - & \\
\hline Einnahmen & 1,4 & 1,3 & 1,1 & 1,0 & 1,2 & 1,1 & 1,0 & 1,3 & 0,8 & 1,2 & 1,1 & 0,6 & 1,3 \\
\hline $\begin{array}{l}\text { sonstige Einnah- } \\
\text { men insgesamt }\end{array}$ & 45,2 & 17,3 & 4,0 & 3,1 & 2,1 & 8,0 & 5,4 & 8,1 & 1,8 & 1,8 & 2,8 & 5,1 & 9,9 \\
\hline
\end{tabular}

Quelle: Bundesministerium der Finanzen =EG-Haushalt 1986= Anlage 10, Bundesministerium der Finanzen =EG-Haushalt $1990=$ Anlage 9 und 10 und Kommission der EG $=$ Haushaltsvademekum $=47$. 


\section{Der Einfluß der Europäischen Gemeinschaften auf die nationalen Ein- nahmensysteme am Beispiel der Bundesrepublik Deutschland}

Die nationalen Einnahmensysteme sind durch die Europäischen Gemeinschaften auf mehrfache Weise betroffen. Zum einen können die nationalen Ebenen ihre Einnahmensysteme nicht mehr völlig frei gestalten, wenn durch die Steuerharmonisierung innerhalb der Europäischen Gemeinschaften ihr Steuerfindungsrecht oder ihr Recht zur Ausgestaltung von Steuern eingeschränkt wird. Entsprechende Regelungen der Europäischen Gemeinschaften berühren die Objekthoheit der Mitgliedstaaten. Zum anderen wird jedoch auch die Ertragshoheit der Mitgliedstaaten betroffen, wenn Einnahmen, die früher den Mitgliedstaaten zustanden, nun an die Europäischen Gemeinschaften abgeführt werden müssen, wie dies z. B. bei den Zöllen der Fall ist. Betroffen sind die Mitgliedstaaten aber auch dann, wenn sie für Einnahmen der Europäischen Gemeinschaften aufkommen müssen, die es vorher in dieser Form nicht gab, denn auch dadurch reduziert sich ihr eigener finanzieller Spielraum. Der Einfluß der Europäischen Gemeinschaften auf die Objekthoheit und Ertragshoheit von Bund und Ländern soll im folgenden genauer untersucht werden.

\section{a) Einschränkungen der Objekthoheit von Bund und Ländern}

Für die Harmonisierung der "indirekten" Steuern bildet Art. 99 EWGV die Grundlage: Der Rat erläßt Bestimmungen zur Harmonisierung der Rechtsvorschriften über die Umsatzsteuer, die Verbrauchsabgaben und sonstige indirekte Steuern, soweit diese Harmonisierung für die Errichtung und das Funktionieren des Binnenmarktes innerhalb der in Artikel 8a EWGV gesetzten Frist ${ }^{31}$ notwendig ist. Für die Errichtung und das Funktionieren des Binnenmarktes ist der Grundsatz der Wettbewerbsneutralität ${ }^{32}$ maßgeblich. Die Steuerharmonisierung in den Europäischen Gemeinschaften steht insofern stets in dem Spannungsverhältnis zwischen der Vermeidung von Wettbewerbsverzerrungen im Handel zwischen den Mitgliedstaaten und der Beschränkung der nationalen Steuerhoheit. ${ }^{33}$

31 D. h. also bis zum 31.12.1992.

32 In Art. 3f EWGV wird die Errichtung eines Systems gefordert, das den Wettbewerb innerhalb des Gemeinsamen Marktes vor Verfälschungen schützt.

33 Über dieses Spannungsverhältnis darf jedoch auch die Frage nach der Steuerkonzeption nicht aus den Augen verloren werden. 


\section{Einnahmenverteilung}

Unterschiedliche Steuersätze der Umsatzsteuer galten bisher als nicht wettbewerbsverzerrend, da durch den Grenzausgleich, d. h. die Rückerstattung der Steuer im Exportland und die steuerliche Belastung im Importland, die Waren im Bestimmungsland unabhängig von ihrer Herkunft mit dem gleichen Satz belastet waren. In jedem Mitgliedstaat wurde der Verbrauch gemäß den dort geltenden Steuersätzen belegt und auch das daraus resultierende Steueraufkommen floß dem betreffenden Staat zu. Die grenzüberschreitende Besteuerung folgte damit dem Bestimmungslandprinzip. ${ }^{34}$ Dies entspricht im übrigen auch einer Belastungskonzeption der Umsatzsteuer als Verbrauchsteuer. ${ }^{35}$ Vor allem hatte das Bestimmungslandprinzip für die einzelnen Mitgliedstaaten den Vorteil, daß sie ihre steuerliche Autonomie weitgehend wahren konnten: Struktur, Niveau und Differenzierung der Belastung konnten nach nationalen Erwägungen gestaltet werden. ${ }^{36}$

Allerdings erforderte dieses System Kontrollen an den Grenzen. Diese sind nicht mit dem Binnenmarkt vereinbar. Die Kommission hat daher Vorschläge zum Abbau der Steuergrenzen ${ }^{37}$ vorgelegt, nach denen Transaktionen zwischen den Mitgliedstaaten genauso behandelt werden wie entsprechende Tatbestände innerhalb der Mitgliedstaaten. Ist der Empfänger im gemeinschaftlichen Handel ein vorsteuerabzugsberechtigter Unternehmer, kann er als Importeur den Vorsteuerabzug anwenden, so daß die Umsatzsteuer des exportierenden Staates als Vorsteuer abgezogen und die Umsatzsteuer des importierenden Staates aufgeschlagen. wird. Soweit es sich um steuerpflichtige Unternehmen handelt, wird damit weiterhin dem Bestimmungslandprinzip entsprochen. Der Wettbewerb wird auch hier nicht verzerrt. "Da der Importeur die ihm in Rechnung gestellte Steuer absetzen kann, bewirken höhere oder niedrigere Steuersätze im Ausland keinen Wettbewerbsvorteil oder -nachteil für die betreffenden Güter." 38 Eine Harmonisierung der Steuersätze wäre damit nicht notwendig.

34 Zu den Prinzipien der Besteuerung im internationalen Handel vgl. Peffekoven =Internationale Finanzordnung $=219 \mathrm{ff}$.

$35 \mathrm{Vgl}$. Pohmer $=$ Grenzausgleich $=256 \mathrm{f}$.

36 Vgl. Parsche, Seidel, Teichmann $=$ Beseitigung von Steuergrenzen $=27$.

37 Vgl. Kommission der EG =Harmonisierung indirekter Steuern 1987=; vgl. dazu im folgenden auch Pflugmann =Steuergrenzen= 324 .

38 Sachverständigenrat zur Begutachtung der gesamtwirtschaftlichen Entwicklung =Jahresgutachten $1989 / 90=$ Ziff. 429. 
Wettbewerbsverzerrungen ergeben sich allerdings bei grenzüberschreitenden Transaktionen mit Empfängern, die nicht umsatzsteuerpflichtig sind, insbesondere mit Endverbrauchern. Hier bleibt die Steuer des Ursprungslandes auf der Ware erhalten. Direktimporte dieser Art werden also entsprechend dem Ursprungslandprinzip belastet. Für Käufe von Kraftfahrzeugen und für den Versandhandel hat die Kommission zwar Sonderregelungen vorgeschlagen, die diese Transaktionen dem Bestimmungslandprinzip unterwerfen, sie hielt die übrigen privaten Direktkäufe jedoch für eine Quelle gravierender Wettbewerbsverzerrungen und hat deshalb auch Vorschläge zur Harmonisierung der Steuersätze gemacht.

Die Vorschläge der Kommission konnten bisher keine Mehrheit finden, so daß für eine begrenzte Zeit (1.1.1993 bis 31.12.1996) eine Übergangsregelung getroffen wurde. Dabei wird der Grenzausgleich an der geographischen Grenze selbst abgeschafft, die Steuerrückerstattungen und die Belastung der Einfuhren werden in die Unternehmen verlagert. Dies soll durch ein Kontrollverfahren, insbesondere durch einen Informationsaustausch auf der Grundlage gespeicherter Daten, realisiert werden. ${ }^{39}$ Auch hier unterliegen Importe durch Letztverbraucher dem Ursprungslandprinzip.

Grundsätzlich liegt bei den nationalen Mehrwertsteuern bisher eine zwischen der Europäischen Gemeinschaft und den Mitgliedstaaten geteilte Objekthoheit vor. Die Gemeinschaft schreibt ein einheitliches Mehrwertsteuersystem und eine einheitliche Bemessungsgrundlage vor. Die Objekthoheit bei der Mehrwertsteuer-Bemessungsgrundlage liegt damit prinzipiell bei den Europäischen Gemeinschaften. Allerdings bleibt den Mitgliedstaaten durch Ausnahmeregelungen noch ein gewisser Spielraum. Vor allem aber konnten die Mehrwertsteuer-Sätze bisher von den Mitgliedstaaten noch frei gewählt werden. Der Vorschlag der Kommission, nur noch einen Normalsatz zwischen $14 \%$ und $20 \%$ und einen ermäßigten Satz zwischen $4 \%$ und $9 \%$ zuzulassen $^{40}$, konnte im Rat keine Mehrheit finden. Schon die Anzahl der Sätze ist in den einzelnen Mitgliedstaaten unterschiedlich, erst recht die Höhe der Sätze (Siehe Tabelle 16). Anscheinend stellt eine solche Harmonisierung einen $\mathrm{zu}$ weitgehenden Eingriff in die nationale Steuerautonomie dar.

39 Vgl. Rat der EG = Mehrwertsteuer-Übergangsregelung 1990=.

40 Vgl. Kommission der EG $=$ Harmonisierung indirekter Steuern $1987=6$. 
Tabelle 16: Die Mehrwertsteuersätze in den Mitgliedstaaten den Europäischen Gemeinschaften im Jahre 1991

\begin{tabular}{|c|c|c|c|c|}
\hline \multirow{2}{*}{$\begin{array}{l}\text { Mitglied- } \\
\text { staat }\end{array}$} & \multicolumn{4}{|c|}{ Steuersätze in \% } \\
\hline & $\begin{array}{c}\text { Normalsatz } \\
\text { Sätze }^{1)}\end{array}$ & $\begin{array}{c}\text { ermäßigte } \\
\text { Sätze }^{2}\end{array}$ & erhöhte & Nullsatz $\left.{ }^{3}\right)$ \\
\hline $\begin{array}{l}\text { B } \\
\text { DK } \\
\text { D } \\
\text { F } \\
\text { GRL } \\
\text { IRL } \\
\text { I } \\
\text { LUX } \\
\text { NL } \\
\text { P } \\
\text { E } \\
\text { UK }\end{array}$ & $\begin{array}{c}19 \\
22 \\
14 \\
18,6 \\
18 \\
21 \\
19 \\
12 \\
18,5 \\
17 \\
12 \\
15\end{array}$ & $\begin{array}{c}1 ; 6 ; 17 \\
\overline{7} \\
2,1 ; 5,5 ; 13 \\
3 ; 8 \\
10 ; 12,5 \\
4 ; 9 \\
3 ; 6 \\
6 \\
8 \\
6 \\
-\end{array}$ & $\begin{array}{c}25 ; 33 \\
- \\
- \\
224) \\
36 \\
- \\
38 \\
- \\
- \\
30 \\
33 \\
-\end{array}$ & $\begin{array}{c}\mathrm{ja} \\
\mathrm{ja} \\
- \\
- \\
- \\
\mathrm{ja}^{5)} \\
- \\
- \\
- \\
\left.\mathrm{ja}^{6}\right) \\
\mathrm{ja}^{5}\end{array}$ \\
\hline \multicolumn{5}{|c|}{$\begin{array}{l}\text { 1) Insbesondere für bestimmte Warengruppen des lebensnotwenigen Bedarfs und für bestimmte Dienst- } \\
\text { leistungen im Sozial- und Kulturbereich. } \\
\text { 2) Für bestimmte Warengruppen des gehobenen Bedarfs. } \\
3 \text { Nullsatz= Steuerbefreiung mit Vorsteuerabzug. } \\
\text { 4) Für Kraftfahrzeuge und einige Güter des gehobenen Bedarfs ab 8.9.1989. } \\
\text { 5) Für Nahrungsmittel, Getränke, Medikamente u. a. } \\
\text { 6) Für Grundnahrungsmittel und landwirtschaftliche Produkte. }\end{array}$} \\
\hline
\end{tabular}

Quelle: Kommission der EG $=$ Steuergrenzen $1990=8$ und Barre $=$ EC Tax Scene $=284$. 
Tabelle 17: Übersicht der speziellen Verbrauchsteuern, die nach den Vorschlägen der Kommission aufgehoben oder neu ein geführt werden müßten

\begin{tabular}{|llc|}
\hline & aufzuhebende Steuer auf & $\begin{array}{r}\text { neu einzuführende } \\
\text { Steuer auf }\end{array}$ \\
\hline Belgien & Mineralwasser, Limonade, Zucker, &.$/$. \\
Dänemark & $\begin{array}{l}\text { Kaffee, Tee, Süßwaren, Speiseeis, Mineralwasser, Parfum, Körperpflegemittel, } \\
\text { Kfz-Registrierung, Zündwaren, Leuchtmittel, Unterhaltungselektronik, Haus- } \\
\text { haltsgeräte, Verpackungen, Spielkarten, Charterflüge, Stein- und Braunkohle }\end{array}$ &.$/$. \\
Deutschland & Kaffee, Tee, Zucker, Salz, Leuchtmittel, Erdgas & Wein \\
Frankreich & Zucker, Tee, Kakao, Kfz-Registrierun \\
Griechenland & Spielkarten, Salz, Kfz-Zusatzverbrauchsteuer \\
Irland & Mineralwasser, Zündwaren, Ersterwerb von Kfz, Fernseh- und Video-Geräte, & Wein \\
Italien & Kchallplatten, Kreditkarten &.$/$. \\
Luxemburg & Zuckee, Kakao, Zucker, Glukose, Pflanzenöl, Margarine, Bananen, Zündhölzer, & Wein \\
Niederlande & Zucker, Mineralwasser, Limonade, Fruchtsaft, Kfz-Zusatzverbrauchsteuer, \\
Portugal & Zündhölzer & Wein aus inl. Trauben \\
Spanien & ./. &.$/$. \\
Vereinigtes & Zündwaren, Ersterwerb von Kfz & Wein \\
Königreich & & Wein \\
\hline
\end{tabular}

Quelle: Wissenschaftlicher Dienst des deutschen Bundestages $=$ Harmonisierung $=19$. 


\section{Einnahmenverteilung}

Für die Bundesrepublik Deutschland hätten die Vorschläge zwar keine Auswirkungen auf die Satzstruktur und die Satzhöhe gehabt, allerdings hätte es Änderungen bei der Zuordnung zu dem ermäßigten Satz gegeben. Nach den Kommissionsvorschlägen müßten z. B. bestimmte kulturelle Leistungen von Theatern, Orchestern etc., die nach $\S 12$ Abs. 2 Nr. 7 UStG nur dem ermäBigten Satz unterliegen, dem vollen Satz unterworfen werden. Andererseits müßte der ermäßigte Satz z. B. auf nichtalkoholische Getränke oder pharmazeutische Erzeugnisse ausgedehnt werden.

Nachdem der Bandbreitenvorschlag keine Mehrheit fand, schlug die Kommission einen Mindestsatz für die Mehrwertsteuer vor. Diesem Vorschlag folgte schließlich auch der Rat, wobei er die ab 1993 gültige Untergrenze für den Normalsatz bei $15 \%$ und für den ermäßigten Satz bei $5 \%$ festlegte. Die "Nullsätze" für bestimmte Güter des lebensnotwendigen Bedarfs dürfen beibehalten werden. ${ }^{41}$ Dies bedeutet für die Bundesrepublik Deutschland, aber auch für Luxemburg und Spanien, daß der Normalsatz angehoben werden muß.

Bei den speziellen Verbrauchsteuern legte die Kommission ebenfalls Vorschläge zur Steuerharmonisierung vor. Nach diesen Vorschlägen wäre das Steuerfindungsrecht der Mitgliedstaaten durch die Europäische Gemeinschaft eingeschränkt worden, da vorgesehen war, daß lediglich auf bestimmte Güter spezielle Verbrauchsteuern erhoben werden dürfen. Vorschläge der Kommission $^{42}$ sahen vor, daß nur noch alkoholische Getränke, Tabakwaren und Mineralöl mit einer speziellen Verbrauchsteuer belegt werden dürfen. Diese Vorschläge wurden allerdings bisher noch nicht vom Rat gebilligt. Dies liegt nicht zuletzt daran, daß die speziellen Verbrauchsteuern innerhalb der Europäischen Gemeinschaften sehr vielfältig sind, so daß die Zahl der aufzuhebenden oder neu einzuführenden Steuern relativ groß ist (Siehe Tabelle 17). Da mit den speziellen Verbrauchsteuern nicht nur fiskalische, sondern auch andere, z. B. gesundheitspolitische Ziele verfolgt werden, würde der nationale Handlungsspielraum auch auf diesen Gebieten eingeschränkt.

Auch für die Harmonisierung der Steuersätze der speziellen Verbrauchsteuern lagen seit 1987 Vorschläge der Kommission vor, wobei auch hier die zunächst

41 Vgl. o. V. =Europäische Steuerangleichung $=15$.

42 Vgl. Kommission der EG = Harmonisierung indirekter Steuern $1987=8$ f. und Kommission der EG $=$ Mehrwertsteuer- und Verbrauchsteuersätze 1987 $=2$. 
vorgesehenen einheitlichen Steuersätze ${ }^{\mathbf{4 3}}$ keine Zustimmung fanden. Die Kommission legte daraufhin Vorschläge vor, die Mindestsätze bzw. Steuermargen sowie längerfristige Zielsätze enthielten. Der Rat beschloß im Juni 1991 auch bei den speziellen Verbrauchsteuern auf alkoholische Getränke, Tabakwaren und Mineralöl Mindestsätze, allerdings mit einigen Ausnahmeregelungen: nicht in die Regelungen einbezogen wurden beispielsweise leichtes Heizöl, Spirituosen und Wein. Die deutschen Steuersätze liegen fast alle über den beschlossenen Mindestgrenzen - als einzige Ausnahme muß die Biersteuer erhöht werden -, so daß sich für die deutschen Verbrauchsteuern keine großen Änderungen ergeben, zumal keine Mindestgrenze für die Besteuerung von Wein festgelegt wurde. 44

Im Zusammenhang mit der Verwirklichung des Binnenmarktes hat auch die Diskussion über die Harmonisierung von Unternehmen- und Kapitalertragsteuern zugenommen. Bisher wurden allerdings lediglich zwei Richtlinien verabschiedet. Eine Richtlinie sieht ein gemeinsames Steuersystem vor für Fusionen, Spaltungen und die Einbringung von Unternehmensteilen, die Gesellschaften verschiedener Mitgliedstaaten betreffen. ${ }^{45}$ Die andere Richtlinie schreibt ein gemeinsames Steuersystem für Mutter- und Tochtergesellschaften verschiedener Mitgliedstaaten vor. ${ }^{46}$

Gewisse Auswirkungen hatten die Harmonisierungsbestrebungen der Europäischen Gemeinschaften in der Bundesrepublik Deutschland insofern, als die Diskussion um die - allerdings ohnehin überfällige - Abschaffung der Börsenumsatzsteuer und der Gesellschaftsteuer u. a. unter dem Aspekt der Steuerharmonisierung in den Europäischen Gemeinschaften geführt wurde. Auch die Harmonisierung der Kapitalertragsteuern wurde in Verbindung mit dem Binnenmarkt verstärkt diskutiert, vor allem mit Blick auf die Liberalisierung des Kapitalverkehrs. Diese Diskussion wurde zunächst durch die Einführung der Quellensteuer in der Bundesrepublik verschärft. ${ }^{47}$ Eine Einigung im Rat konnte auch hier nicht erreicht werden, zumal die Abschaffung der Quellensteuer das Interesse Deutschlands an der Harmonisierung schwächer

43 Vgl. Kommission der EG = Harmonisierung indirekter Steuern 1987=9.

44 Vgl. o. V. =Europäische Steuerangleichung $=15$ und 17.

$45 \mathrm{Vgl}$. Rat der EG =Fusionsrichtlinien 1990= $1 \mathrm{ff}$.

46 Vgl. Rat der EG =Konzernrichtlinien $1990=6 \mathrm{ff}$.

47 Vgl. Schemmel =Steuerharmonisierung $=146$. 


\section{Einnahmenverteilung}

werden ließ und Luxemburg, Großbritannien und Griechenland ohnehin eine Harmonisierung ablehnten. Ein Richtlinienvorschlag der Kommission für eine Quellensteuer vom Frühjahr 1989 stieß im Rat auf Ablehnung. 48

Die erwähnten tatsächlichen und potentiellen Einschränkungen der nationalen Objekthoheit betreffen in der Bundesrepublik Deutschland vor allem den Bund, dem de facto die Objekthoheit für fast alle Steuern in der Bundesrepublik Deutschland obliegt, da er die konkurrierenden Gesetzgebungskompetenzen vollständig an sich gezogen hat. Lediglich bei einer Harmonisierung der örtlichen Verbrauch- und Aufwandsteuern wären die Länder betroffen.

\section{b) Einschränkungen der Ertragshoheit von Bund und Ländern}

Die nationale Ertragshoheit kann in zweifacher Hinsicht durch die Europäischen Gemeinschaften berührt werden. Erstens besteht die Möglichkeit, daß sich mit dem Wegfall des Grenzausgleichs im innergemeinschaftlichen Handel auch die Aufteilung des Steueraufkommens zwischen den Mitgliedstaaten ändert. Wird der Konzeption der Kommission gefolgt, so wird die Umsatzsteuer, die der Exporteur in seinem Staat zu entrichten hat, beim Importeur von der Steuerschuld abgezogen, so daß das Importland ein entsprechend gemindertes Aufkommen erhält. Das bedeutet aber auch, daß dem Mitgliedstaat, in dem der Verbrauch stattfindet, nicht mehr die volle Umsatzsteuer, die auf einem Gut liegt, zusteht. "Die Aufteilung des Steueraufkommens richtet sich einerseits nach der Höhe des Mehrwertes, andererseits aber auch nach der unterschiedlichen Höhe der Steuersätze. "49 Die Kommission hat daher ein Clearing-Verfahren vorgeschlagen, das die ursprüngliche Steueraufteilung sichern soll. Dieses Verfahren ist jedoch mit einigen technischen Schwierigkeiten ${ }^{50}$ verbunden, so daß gewisse Verschiebungen in der Verteilung des Steueraufkommens nicht völlig ausgeschlossen werden können.

Zweitens beruhen schwerwiegende Eingriffe in die nationalen Ertragshoheiten darauf, daß die Mitgliedstaaten zugunsten der Gemeinschaft auf bestehende nationale Einnahmen verzichten müssen. Dies war bisher strenggenommen nur bei den Zöllen der Fall. Die anderen Einnahmen gab es zuvor auf nationaler

48 Vgl. Schemmel = Steuerharmonisierung $=147$.

49 Pflugmann $=$ Steuergrenzen $=329$.

50 Vgl. dazu v. a. Parsche, Seidel, Teichmann $=$ Beseitigung von Steuergrenzen $=433 \mathrm{ff}$. 
Ebene nicht, wie die Agrarabschöpfungen, oder sie besitzen den Charakter von Finanzzuweisungen, deren interne Aufbringung nicht von vornherein an eine bestimmte nationale Einnahmenquelle gebunden ist, wie z. B. die Bruttosozialprodukt-Eigenmittel. Bei den Mehrwertsteuer-Eigenmitteln wird häufig von einer geteilten Ertragshoheit gesprochen. ${ }^{51}$ Doch eine Bestimmung der Europäischen Gemeinschaften, daß Mehrwertsteuer-Eigenmittelzahlungen aus den nationalen Mehrwertsteuer-Einnahmen zu leisten sind, gibt es nicht. "Die EG ist ... nicht Gläubigerin der nationalen Umsatzsteuer - sie hat keinen Anspruch auf Teile der Umsatzsteuer-, sondern sie hat nur einen Anspruch gegen die Mitgliedstaaten auf Zahlung eigener Einnahmen. Mithin sind die Mitgliedstaaten Schuldner der Mehrwertsteuer-Eigenmittel und nicht der einzelne Steuerpflichtige." 52 Die Regelung in der Bundesrepublik Deutschland, nach der die Mehrwertsteuer-Eigenmittel bei den Mehrwertsteuer-Einnahmen des Bundeshaushaltes abgesetzt werden, ist nicht zwingend. Dies zeigt, daß die Mehrwertsteuer-Eigenmittel sich de facto noch nicht sehr weit von Finanzbeiträgen entfernt haben. Der für Steuer charakteristische Tatbestand, daß unentgeltliche Leistungen von Privaten an den Staat fließen, wird bei den MehrwertsteuerEigenmitteln nicht unmittelbar erfüllt. Die Kommission räumt selbst ein, daß es sich bei ihnen "nicht ganz um echte eigene Mittel" handelt. 53

Aus dem Blickwinkel der nationalen Haushalte ist allerdings eine allgemeine Einnahmenkonkurrenz in dem Sinn gegeben, daß sich das für die Finanzierung nationaler Aufgaben verfügbare Finanzvolumen in dem Umfang, in dem Mehrwertsteuer-Eigenmittelzahlungen an die Europäischen Gemeinschaften geleistet werden, verringert. Von einem Verbundsystem, vergleichbar mit der Verteilung der Mehrwertsteuer zwischen Bund und Ländern, kann man zwar rein formal nicht sprechen. ${ }^{54}$ Doch läßt sich in Deutschland eine Art "mittelbares Verbundsystem" feststellen. Der Anteil der Mehrwertsteuer-Eigenmittelzahlungen an den Mehrwertsteuer-Einnahmen weist in der Bundesrepublik

51 Vgl. z. B. Reister $=$ Haushalt der $\mathrm{EG}=35$.

52 Augstein $=$ Finanzwirtschaft der EG $=331$.

53 Kommission der EG = Finanzierung des Gemeinschaftshaushaltes $=17$.

54 Eine Regelung über eine einheitliche Quotenbeteiligung der Gemeinschaftsebene an den nationalen Mehrwertsteuer-Einnahmen in allen Mitgliedstaaten scheitert schon daran, daß das Mehrwertsteuer-Eigenmittelsystem bei den nationalen MehrwertsteuerBemessungsgrundlagen ansetzt, nicht bei den nationalen Mehrwertsteuer-Einnahmen. Vgl. Messal $=$ EG-Finanzierung $=132$. 


\section{Einnahmenverteilung}

Deutschland nur geringe Schwankungen auf. ${ }^{55}$ Insofern existiert hier rein materiell eine "mittelbare Verbundquote". Zwar besitzt die unmittelbare Beteiligung von Bund und Ländern an den Mehrwertsteuer-Einnahmen eine andere Qualität als die der Europäischen Gemeinschaften; um gleichwohl die materiell enge Bindung zwischen Mehrwertsteuer-Eigenmitteln und nationalen Mehrwertsteuer-Einnahmen herauszustellen, könnte man von einer 'QuasiVerbundsbeteiligung' der Europäischen Gemeinschaften an den nationalen Mehrwertsteuer-Einnahmen sprechen. ${ }^{56}$ Dies gilt um so mehr, als der Bund aus seiner Sicht die Verteilung des Mehrwertsteueraufkommens folgendermaßen ausweist:

Tabelle 18: Verteilung des Umsatzsteueraufkommens ${ }^{*}$ in der Bundesrepublik Deutschland im Jahre 1990

\begin{tabular}{|lrrr|}
\hline & in Mrd. DM & $\begin{array}{r}\text { in \% des } \\
\text { Gesamtauf- } \\
\text { kommens }\end{array}$ & $\begin{array}{r}\text { in \% des } \\
\text { Bundesauf- } \\
\text { kommens }\end{array}$ \\
$\begin{array}{l}\text { Gesamtaufkommen } \\
\begin{array}{l}\text { Anteil des Bundes } \\
\text { Ergänzungsanteil }\end{array}\end{array}$ & 141800 & 100,0 & 100,0 \\
$\begin{array}{l}\text { an finanzschwache } \\
\text { Länder }\end{array}$ & 92170 & 65,0 & 3,1 \\
$=\begin{array}{l}\text { Bundesanteil nach } \\
\text { Ergänzungszuweisung }\end{array}$ & 2854 & 2,0 & 96,9 \\
- EG-MWSt-Eigenmittel & 15100 & 63,0 & 16,4 \\
= verfügbarer Bundes- & 74216 & 52,3 & 80,5 \\
\hline anteil & 74216 & & \\
\hline * EinschlieBlich Einfuhrumsatzsteuer. & & & \\
\hline
\end{tabular}

Quelle: Bundesministerium der Finanzen =EG-Haushalt 1990= Anlage 15.

Auch die Einschränkungen der Ertragshoheit betreffen in der Bundesrepublik Deutschland unmittelbar nur den Bund. Er hat die Ertragshoheit an den Zöllen abgetreten, er kommt für die Mehrwertsteuer-Eigenmittelzahlungen der Bundesrepublik Deutschland an die Europäischen Gemeinschaften durch den Verzicht auf einen Teil seiner Mehrwertsteuer-Einnahmen auf und er muß aus sei-

55 Vgl. dazu Messal $=$ EG-Finanzierung $=136$.

56 Vgl. Messal $=$ EG-Finanzierung $=136 \mathrm{f}$. 
nem Haushalt auch die Bruttosozialprodukt-Eigenmittelzahlungen leisten. Theoretisch besteht für den Bund die Möglichkeit, die Belastungen durch die Europäischen Gemeinschaften im Rahmen der Umsatzsteuerverteilung zwischen Bund und Länder geltend zu machen und einen höheren Anteil durchzusetzen. Davon hat er bisher allerdings keinen Gebrauch gemacht. 
Barbara Pflugmann-Hohlstein - 978-3-631-75187-9 Downloaded from PubFactory at 01/11/2019 07:20:05AM

via free access 


\section{KRITISCHE WÜRDIGUNG DER FINANZAUSGLEICHSREGE- LUNGEN IN DEN EUROPÄISCHEN GEMEINSCHAFTEN UNTER BERÜCKSICHTIGUNG DER SITUATION DER BUNDESREPU- BLIK DEUTSCHLAND}

Bei der Darstellung des Finanzausgleichs innerhalb der Europäischen Gemeinschaften wurden schon an verschiedenen Stellen Zweifel an der Zweckmäßigkeit bestimmter Regelungen angedeutet. Im folgenden Teil der Arbeit sollen die in Teil $\mathbf{C}$ dargestellten Regelungen unter den eingangs entwickelten Kriterien für einen föderativen Aufbau eingehend diskutiert werden. Dabei steht der Problemkreis der Beziehungen der Europäischen Gemeinschaften zur Bundesrepublik Deutschland insgesamt im Vordergrund. Die grundsätzlich für alle Mitgliedstaaten ähnlich gelagerten Probleme ergeben sich selbstverständlich auch für die Bundesrepublik Deutschland. Dazu kommen allerdings noch jene spezifischen Schwierigkeiten, die sich aus dem föderativen Aufbau der Bundesrepublik Deutschland ergeben.

\section{Die mangelnde Berücksichtigung der Vorteile eines föderativen Auf- baus}

\section{Beachtung des Subsidiaritätsprinzips auf der Gemeinschaftsebene?}

Zunächst muß die Frage gestellt werden, ob die Vorteile eines föderativen Aufbaus in den Europäischen Gemeinschaften genügend Beachtung finden. Die Kommission vertritt formal die Ansicht, daß ein konkretes Tätigwerden der Gemeinschaft im Rahmen der Verträge nur dann erfolgen solle, wenn die Gemeinschaft die Aufgabe "besser" lösen kann als die nationalen Ebenen. ${ }^{1}$ Der Grundsatz der Subsidiarität taucht seit geraumer Zeit verstärkt in Gemeinschaftsdokumenten auf. Im Rahmenprogramm für Forschungs- und Technologiepolitik wurde festgeschrieben, daß Gemeinschaftsaktionen nur in den Fällen zu rechtfertigen sind, "in denen sie im Vergleich zu nationalen und anderen internationalen Tätigkeiten ... hinsichtlich der Effizienz und der Finanzierung oder aus wissenschaftlicher und technischer Sicht kurz-, mittel- oder langfristig Vorteile (grösseren Nutzen) aufzuweisen haben"2. Im sozialpolitischen Ak-

1 Vgl. dazu Kommission der EG =Europäische Union = 11 und Delors $=$ Treffen mit den Ministerpräsidenten $=341$.

$2 \quad$ Rat der EG = Rahmenprogramm 1987-1991 = 23. 
tionsprogramm der Kommission vom November 1989 wird in der Einleitung explizit von der "Anwendung des Subsidiaritätsprinzips" gesprochen. ${ }^{3}$ Für die Umweltpolitik wurde das Subsidiaritätsprinzip durch die Einheitliche Europäische Akte sogar in den Verträgen verankert: Nach Art. 130r Abs. 4 EWGV soll die Gemeinschaft nur dann umweltpolitisch tätig werden, wenn die Ziele der Umweltpolitik auf der Gemeinschaftsebene besser erreicht werden können als auf der Ebene der Mitgliedstaaten. Außerdem soll das Subsidiaritätsprinzip nach dem Willen des Europäischen Rates beim Aufbau der Europäischen Wirtschafts- und Währungsunion ${ }^{4}$ und bei der Entwicklung zur Politischen Union ${ }^{5}$ beachtet werden.

Tatsächlich ist jedoch in vielen Bereichen eine Tendenz zur Zentralisierung von Aufgaben auf der Gemeinschaftsebene festzustellen. Dieser "Kompetenzsog" weg von den nationalen Ebenen hin zu den Gemeinschaftsorganen hat in den letzten Jahren, insbesondere im Hinblick auf Maßnahmen zur Verwirklichung des Binnenmarktes, stark zugenommen. Dies gilt sowohl für die von den Europäischen Gemeinschaften aufgegriffenen Aufgabenfelder insgesamt, als auch für die Verteilung der Kompetenzen zwischen den Europäischen Gemeinschaften und nationalen Ebenen im Rahmen der "konkurrierenden" Aufgaben.

Bei manchen Tätigkeitsbereichen der Europäischen Gemeinschaften drängt sich besonders stark die Frage auf, ob die Vorteile eines dezentralen Aufbaus gesehen werden, nämlich dann, wenn die Europäischen Gemeinschaften Aufgaben an sich ziehen, für die bisher - entsprechend der Aufgabenverteilung in der Bundesrepublik Deutschland - die Länder zuständig waren. Auch in der Bundesrepublik Deutschland wird vom Subsidiaritätsprinzip ausgegangen; ${ }^{6}$ wenn also Aufgaben bei den Ländern verblieben sind, so ist zu vermuten, daß die Aufgaben dort durch größere Bürgernähe besser erfüllt werden können als auf Bundesebene. Eine Verlagerung solcher Aufgaben auf die Gemeinschaftsebene muß besonders kritisch betrachtet werden.

3 Kommission der EG = Soziale Grundrechte $=4$.

4 Europäischer Rat $=$ Schlußfolgerungen $1989=607$.

5 Europäischer Rat $=$ Schlußfolgerungen $1990=1333$.

6 Das Subsidiaritätsprinzip liegt dem Grundgesetz zumindest mittelbar zugrunde. Vgl. Dürig =Kommentar = Art. 1 Abs. II Rdnr. 54 . 
Hier wird ein grundsätzliches Problem der Konkretisierung des Subsidiaritätsprinzips auf Gemeinschaftsebene deutlich: Das Subsidiaritätsprinzip geht von der Fähigkeit zur Problemlösung der unterschiedlichen Ebenen aus. Wenn sich jedoch nachgeordnete Instanzen in einem Mehr-Ebenen-Modell auf unterschiedlichen Entwicklungsniveaus befinden, "dann führt die Frage nach der Fähigkeit zur Problemlösung zu keiner eindeutigen Aufgabenzuordnung auf unterschiedliche Ebenen"7. Dies gilt in den Europäischen Gemeinschaften sowohl für die Mitgliedstaaten als auch für die Regionen.

Daraus kann jedoch nicht geschlossen werden, daß den Europäischen Gemeinschaften all jene Aufgaben zu übertragen wären, die etwa von der griechischen Regierung nicht erfüllt werden können. Das Subsidiaritätsprinzip muß vielmehr so interpretiert werden, daß Aufgaben, die grundsätzlich auf einzelstaatlicher Ebene am besten gelöst werden können, langfristig nicht auf die Gemeinschaft übertragen werden dürfen. Können weniger entwickelte Mitgliedstaaten diese Aufgaben nicht bewältigen, sollten sie von der Europäischen $\mathrm{Ge}-$ meinschaft dabei solange unterstützt werden, bis sie selbst dazu in der Lage sind. Eine vollständige und dauerhafte Übernahme dieser Aufgaben durch die Gemeinschaft würde dagegen zu langfristigen Effizienzverlusten führen. ${ }^{8}$ Allerdings dürfen solche Unterstützungen nicht zu Dauereinrichtungen werden. Sie müssen vielmehr so konzipiert werden, daß für den Mitgliedstaat ein Anreiz zur Selbsthilfe bestehen bleibt.

In den folgenden Abschnitten wird gezeigt, daß das Subsidiaritätsprinzip innerhalb der Europäischen Gemeinschaften nicht immer beachtet wird. Den Vorteilen einer dezentralen Wahrnehmung von Aufgaben wird häufig zu wenig Bedeutung beigemessen. Zur Beurteilung der Aufgabenverteilung wird auf die im Teil B zur Konkretisierung des Subsidiaritätsprinzips herangezogenen Kriterien zurückgegriffen.

$7 \quad$ Starbatty, Vetterlein $=$ Technologiepolitische Aufgabenteilung $=97$.

8 So auch Starbatty, Vetterlein $=$ Technologiepolitische Aufgabenteilung $=97$. 
2. Die Problematik der unterschiedlichen Präferenzen in den Europäischen Gemeinschaften

\section{a) Grundsätzliches zur Berücksichtigung unterschiedlicher regionaler Präferenzen}

Hinter dem Subsidiaritätsprinzip steht vor allem die Überlegung, daß die Zentralisierung von Aufgaben auf der Gemeinschaftsebene zu einer geringeren Berücksichtigung unterschiedlicher regionaler Präferenzen und damit zu Wohlfahrtsverlusten führt. Dieses Argument ist für die Europäische Gemeinschaft von großer Bedeutung, da die Präferenzen innerhalb der Gemeinschaft in der Regel heterogener sind als innerhalb eines Mitgliedstaates, so daß es bei einer zentralen Wahrnehmung von Aufgaben auch in verstärktem Maße zu "frustrierten Minderheiten" kommt.

Die Unterschiede in den Präferenzen haben vielfältige Ursachen. Einmal existieren unterschiedliche kulturelle Traditionen, zum anderen beruhen die unterschiedlichen Beurteilungen in den Mitgliedstaaten auch auf verschiedenartigen geschichtlichen Erfahrungen. So wird z. B. die Gefahr einer Inflation von der deutschen Bevölkerung stärker gefürchtet als in den meisten anderen Mitgliedstaaten. Unterschiedliche Einschätzungen können in manchen Politikbereichen auch einfach auf geographische Gegebenheiten zurückgeführt werden. So ergeben sich beispielsweise Differenzen in der Bewertung von Umweltbelastungen aus der unterschiedlichen Regenerationsfähigkeit der Natur in den verschiedenen Mitgliedstaaten. ${ }^{9}$ Unterschiede in den Präferenzen können schließlich auch sozioökonomische Ursachen haben. Je nach Wohlstandsniveau eines Mitgliedstaates wird bestimmten Fragen ein unterschiedliches Gewicht beigemessen werden.

\section{b) Konsensfindungskosten}

Die heterogenen Präferenzen in den Europäischen Gemeinschaften führen häufig zu hohen Konsensfindungskosten. Im Entscheidungsprozeß des Rates finden sich alle drei von Kirsch und Theiler herausgearbeiteten Formen von Kon-

9 Vgl. dazu Weinstock $=$ Europäische Umwelt $=23 \mathrm{f}$. 
sensfindungskosten. So weichen die Mitgliedstaaten zum Teil sehr weit von ihren Optimalvorstellungen ab, um überhaupt zu einer Einigung zu kommen. ${ }^{10}$

Auch Konsensfindungskosten der zweiten Kategorie lassen sich im Entscheidungsprozeß der Europäischen Gemeinschaften feststellen: Häufig werden sogenannte "package-deals" geschnürt. Dabei werden von der Sache her höchst unterschiedliche Probleme und die ihnen zugedachten Lösungen so zu einer Gesamtheit verknüpft, daß für jeden Mitgliedstaat Leistung und Gegenleistung, sorgsam ausbalanciert, einander entsprechen. Ein solches Verfahren des "logrolling" kompliziert Entscheidungen in mehr als einer Hinsicht:

"Die Vorschläge stehen oft in einem losen und manchmal in gar
keinem Zusammenhang. Eine Revision einer schon verabschiede-
ten Angelegenheit ist häufig aufgrund neuer Informationen eigent-
lich erforderlich, jedoch kann sie nicht vorgenommen werden, da
dann das ganze Paket in Frage gestellt würde. Das bedeutet, daß
es extrem schwierig ist, einmal getroffene Entscheidungen zu kor-
rigieren. Hinzu kommt, daß Vorschläge nicht selten in Form von
inhaltlich vagen Deklarationen verabschiedet werden, die später zu
Interpretationsschwierigkeiten führen."

Schließlich findet sich auch die dritte Kategorie von Konsensfindungskosten in Form von Zeit, Nerven und Engagement. Dies wird einerseits durch die z. T. sehr lange Dauer zwischen der Vorlage und der Entscheidung bei Rechtsakten, andererseits durch die Marathonsitzungen, die der Ministerrat abhält, deutlich. ${ }^{12}$

Die Konsensfindungskosten der Europäischen Gemeinschaften waren besonders hoch, solange de facto das Einstimmigkeitsprinzip galt. ${ }^{\mathbf{1 3}}$ Durch die Einheitliche Europäische Akte wurde für viele Bereiche der ursprünglich in den Verträgen vorgesehene, seit 1966 aber durch den "Luxemburger Kompromiß" außer Kraft gesetzte ${ }^{14}$ Mehrheitsbeschluß wieder eingeführt. Dadurch wurde zwar der Entscheidungsprozeß beträchtlich beschleunigt und vereinfacht, je-

10 Ein Beispiel dafür stellt die Initiative der deutschen Regierung zur Einführung abgasärmerer Autos 1983-1985 dar, bei der die Regierung immer größere Abstriche von ihrer Position machen mußte.

11 Faber, Breyer $=$ Konstitutionelle Aspekte $=218 \mathrm{f}$.

12 Vgl. Faber, Breyer $=$ Konstitutionelle Aspekte $=218 \mathrm{f}$.

13 Teilweise waren die Konsensfindungskosten so hoch, daß sie die Vorteile einer gemeinsamen Handlung überstiegen. Dann endeten die Verhandlungen in einer Sackgasse.

14 Vgl. dazu u. a. Harbrecht $=E G=95 f$. 
doch erhöhte sich für das einzelne Mitgliedsland die Gefahr, daß Entscheidungen getroffen werden, die nicht in seinem Sinne sind.

\section{c) Institutionelle Mängel}

Die Tatsache, daß die wichtigen Entscheidungen der Europäischen Gemeinschaften auf Vorschlag von Fachbeamten der Kommission im Ministerrat getroffen werden, führt zu demokratischen Defiziten ${ }^{15}$, die unter dem Blickwinkel der Föderalismustheorie durchaus als Kosten im Sinne von Nutzeneinbußen der Bürger betrachtet werden können. Für die Bürger ist es sehr viel schwieriger, ihre Interessen deutlich zu machen und durch ein bestimmtes Wahlverhalten zu wahren als auf nationaler Ebene. Direkt wählen können sie lediglich das Europäische Parlament. Doch während in vielen Bereichen die nationalen Parlamente nicht mehr zuständig sind, hat das Europäische Parlament bisher nur begrenzte Einflußmöglichkeiten erhalten. Der Ministerrat beschließt Gesetze hinter verschlossenen Türen und entzieht sich der direkten Beurteilung durch den Bürger schon allein durch die Tatsache, daß nationale Minister nicht einfach abgewählt werden können. Die Kommission stellt für den Bürger häufig eine ferne, in demokratische Mechanismen nicht sichtbar eingebundene Bürokratie dar. ${ }^{16}$ Ihre Arbeit kann durch ihn letztlich überhaupt nicht kontrolliert werden.

Die politische Macht liegt damit weitgehend in der Hand derer, die keinem Wahlmechanismus unterliegen und sich für ihr politisches Tun nicht vor den Wählern verantworten müssen. Die Wähler verlieren angesichts der für sie undurchsichtigen Verteilung politischer Macht häufig das Interesse für politische Probleme. ${ }^{17}$ Eine an den Präferenzen der Bürger ausgerichtete Politik wird dadurch erschwert, und diese "Präferenzkosten" dürften in den Europäischen Gemeinschaften nicht zu vernachlässigen sein.

Mit der geringen Bürgernähe tritt noch ein weiteres Problem auf: Nicht alle Interessen haben die gleichen Chancen gehört zu werden, denn gut organisierte Interessenvertretungen haben normalerweise einen besseren Zugang zum poli-

15 Graf Vitzthum spricht von "parlamentarischen Kosten". Vitzthum =Föderalismus= 285.

16 Vgl. Kielmansegg $=$ Weg zur Politischen Union $=13$.

17 Vgl. dazu Fischer =Fluch des Kollektivs $=84 \mathrm{ff}$, hier insbesondere 87, der dieses Problem allerdings nur für die Kompetenzverflechtung in der Bundesrepublik erörtert. 
tisch-administrativen Entscheidungsträger. Dies gilt schon für den nationalen Bereich, durch die größere Entfernung zum Bürger jedoch noch weit mehr für die Europäischen Gemeinschaften. Die komplexen und intransparenten Entscheidungsprozesse auf Gemeinschaftsebene können zu ganz spezifischen "Interessenfilterungen" führen. Die Bedeutung des Lobbyismus läßt sich nicht zuletzt an der großen Zahl von Büros ("Lobbyfilialen") in Brüssel ablesen. ${ }^{18}$ Es besteht insofern bei einer Zentralisierung von Aufgaben nicht nur die Gefahr, daß die unterschiedlichen Interessen der Bürger nicht beachtet werden, sondern daß nicht einmal den Präferenzen der Mehrheit entsprochen wird, da die Kompetenzverlagerung für die Durchsetzung gut organisierter Einzelinteressen mißbraucht werden kann. ${ }^{19}$

Grundvoraussetzung für die Berücksichtigung der unterschiedlichen Interessen der Bürger ist, daß die Entscheidungen im Parlament - als dem einzigen demokratisch legitimierten Organ - getroffen werden. Dadurch werden die Entscheidungsprozesse für die Bürger transparenter, und nur so können die Bürger überhaupt durch Wahlen Einfluß nehmen. Auch die mit dem Entscheidungsfindungsprozeß im Rat verbundenen Probleme, wie z. B. das Verfahren des "log-rolling", könnten so vermieden werden.

In Anbetracht der Überlegungen zu der Berücksichtigung unterschiedlicher regionaler Präferenzen und zu den Konsensfindungskosten ist eine Verlagerung von Aufgaben auf die Gemeinschaft nur dann sinnvoll, wenn gewichtige Vorteile die Nachteile einer Zentralisierung wettmachen können.

\section{3. Überlegungen zu einzelnen Aufgabenbereichen}

Die Übertragung von Aufgaben auf die Gemeinschaft bietet dann Vorteile, wenn bei der Bereitstellung öffentlicher Güter auf Gemeinschaftsebene Skalenerträge genutzt werden können oder wenn grenzüberschreitende SpilloverEffekte auftreten, bei denen ein Ausgleich auf horizontaler Ebene nicht möglich ist.

18 Vgl. dazu o. V. = Pfadfinder im EG-Labyrinth $=18$.

19 Vgl. dazu Weidner, Knoepfel =Implementierungschancen $=62$. 


\section{a) Die Umweltpolitik}

Eine Zuständigkeit der Europäischen Gemeinschaften für die Umweltpolitik bei grenzüberschreitenden Umweltbelastungen scheint zunächst ein gutes Beispiel für eine solche Argumentation zu sein. Die Ausdehnung bestimmter Umweltmedien über den Raum entspricht nur in den seltensten Fällen den verwaltungstechnischen Grenzen, auch nicht immer den Staatsgrenzen. ${ }^{20}$ Entsprechende grenzüberschreitende Umweltbelastungen stellen nichts anderes als negative Spillover-Effekte dar. Dabei lassen sich grundsätzlich zwei Fälle unterscheiden: erstens Einwegbelastungen und zweitens reziproke transnationale Umweltbelastungen.

Bei Einwegbelastungen führen die Emissionen eines Staates in einem oder mehreren anderen Staaten zu Belastungen, aber nicht umgekehrt. ${ }^{21}$ Dies ist vor allem bei Flüssen der Fall; in Europa ist das beste Beispiel der Rhein. Wälzt ein Staat am Oberlauf des Flusses einen Teil seiner "Umweltkosten" auf den Nachbarstaat ab, der flußabwärts liegt, indem belastende Stoffe in den Fluß eingeleitet werden, liegen negative Spillover-Effekte vor. Der am Oberlauf liegende Staat hat unter der ausschließlichen Berücksichtigung seiner eigenen Interessen keine Veranlassung zur Verminderung der Verschmutzung. "The export of pollutants does not affect the environmental quality of the area and the regional authority has no incentive to include exported pollutants in its calculations." 22

Der betroffene Nachbarstaat kann versuchen, den Verursacher in bilateralen Verhandlungen dazu zu bewegen, seine die Verschmutzung verursachende Aktivität zu reduzieren. Er wird jedoch nur erfolgreich sein, wenn er dem verursachenden Staat Kompensationszahlungen anbietet. Auf diese Weise kann zwar eine effizientere Lösung erreicht werden, die allerdings nicht gängigen Gerechtigkeitsvorstellungen entspricht. Nach diesen müßte der Verursacher dem betroffenen Staat Entschädigungen für die durch ihn verursachten Schäden zahlen. Freiwillige Zahlungen des Verursachers sind jedoch nicht zu erwarten, solange keine übergeordnete Instanz das Verursacherprinzip garantiert und entsprechende Kompensationen überwacht. Es ist daher notwendig, in einer umfassenderen Gemeinschaft gemeinsame Regeln zu vereinbaren, deren Ein-

20 Vgl. Siebert $=$ Environmental Allocation $=505$.

21 Vgl. Walter $=$ Pollution $=141 \mathrm{f}$.

22 Siebert $=$ Environmental Allocation $=505$. 
haltung auch vor dem Europäischen Gerichtshof eingeklagt und von der Kommission durchgesetzt werden kann. 23

Bei reziproken transnationalen Umweltbelastungen ist die Problematik ein wenig anders gelagert: Hier gibt ein Staat zwar auch Emissionen an andere ab, wird aber gleichzeitig durch die Emissionen dieser Staaten belastet. ${ }^{24}$ Dies ist beispielsweise bei der Verschmutzung der Luft oder der Meere mit mehreren Anrainerstaaten, wie etwa der Nordsee, der Fall. Da in diesem Fall die verschmutzenden Staaten gleichzeitig Betroffene der Umweltbelastungen sind, ist davon auszugehen, daß sie schneller bereit sein werden, kooperativ an einer Lösung $\mathrm{zu}$ arbeiten. ${ }^{25} \mathrm{Je}$ mehr Staaten allerdings beteiligt sind und je weniger genau die Verursacher identifiziert werden können, wie z. B. bei der Luftverschmutzung, desto eher stellt sich die Trittbrettfahrer-Problematik; d. h. jeder Staat hofft, daß er von den Umweltanstrengungen der anderen profitiert, ohne selbst Maßnahmen zu ergreifen. Dann ist es durchaus sinnvoll, durch die höhere Ebene, in unserem Fall die Europäischen Gemeinschaften, Umweltnormen festzulegen.

Die umweltpolitischen Maßnahmen der Europäischen Gemeinschaften, die sich auf die grundsätzlichen Rahmenbedingungen (etwa die Beachtung des Verursacherprinzips) oder bei multilateralen Problemen, wie bei der Luftverschmutzung oder beim Schutz der Nordsee, auch auf die Festlegung konkreter Umweltnormen beziehen, sind also durchaus gerechtfertigt.

Es ist jedoch zu beachten, daß es beim Umweltschutz vielfältige Interessenunterschiede zwischen den Mitgliedstaaten gibt. So ergeben sich von vornherein Differenzen bei der Bewertung von Umweltbelastungen aus der unterschiedlichen Regenerationsfähigkeit der Natur in den einzelnen Staaten. ${ }^{26}$ Während die Flüsse in Großbritannien außerordentlich kurz sind und ihre Regenerationsfähigkeit daher groß ist, weisen die Kontinentalgewässer aufgrund ihrer relativen Länge einen hohen Verschmutzungsgrad auf. Daher sprachen sich die Kontinentalstaaten für restriktivere Emissionsnormen aus, während Großbritannien lockere Immissionsnormen befürwortet. ${ }^{27}$ Neben diesen natürlichen

23 Vgl. Klemmer $=$ Harmonisierung der Umweltpolitik $=267 \mathrm{f}$.

24 Vgl. Dierkes, Hartje $=$ Internationale Aspekte $=98$.

25 Vgl. Dierkes, Hartje $=$ Internationale Aspekte $=98$.

26 Vgl. Knödgen, Sprenger $=U$ Umweltschutz $=30$.

$27 \mathrm{Vgl}$. Weinstock $=$ Europäische Umwelt $=23 \mathrm{f}$. 
Gegebenheiten spielt auch die industrielle Struktur und die Bevölkerungsdichte eines Staates eine Rolle für den Stellenwert der Umweltpolitik. So nimmt beispielsweise das dünner besiedelte Frankreich häufig eine andere Haltung ein als die Bundesrepublik Deutschland. "In dem weithin dünn besiedelten Südwesten Frankreichs hat z. B. eine 'wilde' Müllkippe eine andere Bedeutung, als in der ausgeglichen besiedelten Bundesrepublik. "28

Die unterschiedlichen Interessen der Mitgliedstaaten schlagen sich in den Verhandlungen des Rates nieder. Da nach Art. 130s EWGV der Rat einstimmig über umweltpolitische Fragen entscheiden muß, führen die Entscheidungen des Rates zu Lösungen auf dem kleinsten gemeinsamen Nenner. Damit wird in der Regel nur ein geringer Mindestschutz zu erzielen sein. Nach Art. 130t EWGV werden die einzelnen Mitgliedstaaten grundsätzlich nicht daran gehindert, verstärkte Schutzmaßnahmen beizubehalten oder zu ergreifen, allerdings nur, wenn diese Maßnahmen mit dem Vertrag zur Gründung der Europäischen Wirtschaftsgemeinschaft vereinbar sind. Hinter diesem Zusatz verbergen sich vor allem Restriktionen im Hinblick auf den Gemeinsamen Markt und damit auf die Vermeidung von Wettbewerbsverzerrungen.

$\mathrm{Da}$ im Grunde jede Umweltvorschrift Auswirkungen auf den Wettbewerb hat, muß entschieden werden, welche Störungen noch hingenommen werden können und welche nicht. Dabei kann nicht von vornherein davon ausgegangen werden, daß nur einheitliche Emissionsnormen Wettbewerbsverzerrungen vermeiden. Vielmehr ist zu bedenken, daß voneinander abweichende nationale Umweltbestimmungen auch die Folge divergierender nationaler Immissionsgegebenheiten (wie die Assimilationskapazität der Natur, Besiedlungsdichte, Industriestrukturen etc.) sind. Diese müssen als Teil der Standortbedingungen betrachtet werden. Kostenunterschiede, die auf unterschiedlichen Standortbedingungen beruhen, sollen durchaus wettbewerbswirksam werden. Eine Anpassung an die Standortfaktoren sichert eine effiziente Anordnung der Güterproduktion im Raum. So ist beispielsweise nicht einzusehen, warum in wirtschaftlich unterdurchschnittlich entwickelten Gebieten mit bisher geringer Emissionstätigkeit und noch nicht ausgelasteter natürlicher Selbstreinigungskapazität der Umweltmedien die gleichen Emissionsminderungsanforderungen erfüllt werden müssen wie in hoch verdichteten Gebieten. Damit würde man diesen Regionen von vornherein verbieten, ihre natürlichen und siedlungsstruktu-

28 Weinstock $=$ Europäische Umwelt $=6$. 
rellen Standort- und Wettbewerbsvorteile wahrzunehmen. ${ }^{29}$ Einer ungehemmten Harmonisierung ist daher hier entgegenzutreten. Es genügt, Höchstimmissionsstandards festzulegen, die von nationalen und regionalen Entscheidungsträgern aber durchaus restriktiver ausgestaltet werden können.

Besondere Schwierigkeiten hinsichtlich eines ungestörten Wettbewerbs ergeben sich bei restriktiveren nationalen produktbezogenen Umweltnormen. ${ }^{30}$ Diese werden in der Regel sofort von anderen Mitgliedstaaten als nicht-tarifäre Handelshemmnisse betrachtet. Wird dieser Sichtweise gefolgt, müßten die umweltbedingten Produktanforderungen vollständig harmonisiert werden. Die Präferenzen der Bürger in den Regionen, die restriktivere Umweltschutzvorschriften wollen, kämen nicht zum Zuge; umweltpolitische Fortschritte in diesen Mitgliedstaaten würden gehemmt. Eine solche Einschränkung der nationalen Handlungsfähigkeit entspricht nicht dem Subsidiaritätsprinzip.

Der beschriebene Konflikt zwischen nationaler Autonomie und der Harmonisierung von Rechtsvorschriften durch die Europäischen Gemeinschaften betrifft nicht nur die Umweltpolitik, sondern stellt ein generelles Problem bei der Verwirklichung des Binnenmarktes dar.

\section{b) Die Schaffung des Binnenmarktes}

Die Vorteile eines Binnenmarktes liegen auf der Hand. Sie ergeben sich vor allem aus dem Abbau von Schranken für den innergemeinschaftlichen Handel, aus der Beseitigung von Grenzkontrollen, aus der Nutzung von Größenvorteilen in der Produktion sowie aus dem verstärkten Wettbewerb. ${ }^{31}$ Eine solche Veränderung der Rahmenbedingungen des wirtschaftlichen Handelns ist aus der Sicht des einzelnen Mitgliedslandes nur im Verbund mit anderen sinnvoll, da jeder Mitgliedstaat befürchten muß, daß der Nachbar von der Öffnung des eigenen Marktes profitiert, seinen Markt dagegen nicht öffnet. Diese Aufgaben können daher nur gemeinsam (oder zentral) wahrgenommen werden.

Beim Abbau von Hemmnissen, insbesondere technischer Art - denn diese wirken ja als nicht-tarifäre Handelshemmnisse - wurden zwei unterschiedliche Wege heftig diskutiert. Die Verfechter des Liberalisierungsgedankens vertre-

29 Vgl. Klemmer $=$ Harmonisierung der Umweltpolitik $=266 \mathrm{f}$.

30 Vgl. dazu Knödgen, Sprenger $=$ Umweltschutz $=46$.

31 Vgl. dazu v. a. Cecchini $=$ Europa ' $92=$. 
ten die Auffassung, daß es genüge, wenn im Wege der gegenseitigen Anerkennung jedem Produkt, das in einem anderen Mitgliedsstaat rechtmäßig hergestellt oder vertrieben wird, der Zugang zum heimischen Markt gestattet wird. Den Vertretern des Harmonisierungsprinzips erscheint dies zu kurz zu greifen; sie wollen die technischen Vorschriften vereinheitlichen. ${ }^{32}$

Die Kommission sah im Weißbuch zur Vollendung des Binnenmarktes vor, beide Strategien entsprechend dem Grundsatz zu verbinden: so wenig Harmonisierung wie möglich, so viel Harmonisierung wie nötig. ${ }^{33}$ In der Realität wird die Gemeinschaft diesem Anspruch nicht gerecht.

Einerseits zeigt ein Blick auf die Verkehrspolitik, daß sinnvolle Harmonisierungsmaßnahmen nicht immer getroffen werden. Die vorhandenen Verkehrsanlagen sind im wesentlichen auf die nationalen Bedürfnisse zugeschnitten; die Bedürfnisse des grenzüberschreitenden Verkehrs standen und stehen dabei oft im Hintergrund. Mit zunehmendem Binnenhandel muß jedoch auch das Interesse der Gemeinschaft steigen, bei der Beseitigung von Verkehrsengpässen und bei Vorhaben, die eine starke Auswirkung auf den Handel zwischen den Mitgliedstaaten haben, tätig zu werden. ${ }^{34}$ Damit der Verkehr nicht zu einem Engpaß für den Europäischen Binnenmarkt wird, muß dem Binnenmarktkonzept eine europaweit orientierte Verkehrspolitik zur Seite stehen. ${ }^{35}$ Nur mit einem gesamteuropäischen Verkehrskonzept könnte vermieden werden, daß z. B. huckepackverladene deutsche Lastwagen nicht durch italienische Eisenbahntunnels passen, belgische und französische Loks mit unterschiedlichem Strom fahren, und die Flugüberwachung Europas in 42 Kontrollzentren mit 22 verschiedenen technischen Systemen arbeitet. ${ }^{36}$ Hier wären Maßnahmen der Harmonisierung sinnvoll.

32 Vgl. dazu auch Dichtl = Europäischer Binnenmarkt $=335$.

33 Die Kommission sah vor, die Harmonisierung von Rechtsvorschriften künftig darauf zu beschränken, zwingende Erfordemisse für Gesundheit und Sicherheit festzulegen, die dann in das Recht aller Mitgliedștaaten übernommen werden müssen. In allen anderen Fällen sollte das sogenannte Äquivalenzprinzip gelten: Ein Produkt, das in einem Mitgliedstaat rechtmäßig hergestellt und in den Verkehr gebracht worden ist, ist in allen anderen Mitgliedstaaten ebenfalls zum Verkauf zugelassen. Vgl. Kommission der $\mathrm{EG}=$ Vollendung des Binnenmarktes $=18$.

$34 \mathrm{Vgl}$. Beutler u. a. =Europäische Gemeinschaft $=470$.

35 Vgl. Sachverständigenrat zu Begutachtung der gesamtwirtschaftlichen Entwicklung =Jahresgutachten 1990/91 = Ziff. 465.

36 Vgl. Hirn, Stimpfel $=$ Stau ohne Grenzen $=52 \mathrm{ff}$. 
Andererseits zeigt sich bei nebensächlicheren Fragen eine Tendenz zur Überreglementierung 37 - auch in Detailfragen -, die z. T. schon grotesk wirkt. So wird z. B. in der Richtlinie zur "Harmonisierung der Anforderungen zum Schutz von Sicherheit und Gesundheit für den Verkehr mit Spielzeug"38 über mehrere Seiten detailliert etwa die Entflammbarkeit von Nikolausbärten und die notwendige Bezeichnung von Wasserbällen (Vorschlag der Kommission: Nur in flachem Wasser unter Aufsicht verwenden!) geregelt. Ein weiteres Beispiel ist die Richtlinie "Zur Angleichung der Rechtsvorschriften über den zulässigen Schallpegel bei Rasenmähern", bei der zunächst ein Rasenmäher als "jede motorbetriebene Vorrichtung, die speziell für die Pflege grasbewachsener Flächen für Freizeit-, Dekorations- oder ähnliche Zwecke durch Schneiden, ungeachtet des Schneidemechanismus" definiert wird. ${ }^{39}$

Der Einwand, daß es häufig produktbezogene nationale Regelungen gibt, die als nicht-tarifäre Handelshemmnisse wirken, und daher eine Harmonisierung notwendig sei, trifft bei solchen unwichtigen Regelungen nicht zu. Das Problem der nicht-tarifären Handelshemmnisse ließe sich eben auch durch die gegenseitige Anerkennung der Normen nach dem Äquivalenzprinzip beseitigen. Auch hier genügt es also, daß die Gemeinschaft den Rahmen setzt; einzelne Detailregelungen sind nicht nötig.

In den Europäischen Gemeinschaften scheint sich jedoch Niskanens Theorie der Bürokratie auf besonders eindrucksvolle Weise zu bestätigen. Seine These, daß der einzelne Bürokrat ein Interesse daran hat, daß sich der Umfang öffentlicher Aktivitäten vergrößert, da ihm dies Vorteile in Form von Macht, Prestige etc. bringt, ${ }^{40}$ scheint hier voll zuzutreffen. Ansehen und Beförderungschancen von Mitarbeitern der Bürokratie hängen vor allem von der Zahl der vorbereiteten Rechtsakte ab. Daher werden auf allen erdenkbaren Gebieten Richtlinienvorschläge erarbeitet. ${ }^{\mathbf{4 1}}$

Die oben genannten Vorteile des Binnenmarktes erlauben es jedenfalls nicht, ein solches $\mathrm{Ma} ß$ an Harmonisierung von Rechtsvorschriften zu rechtfertigen.

371989 wurden insgesamt 5739 Rechtsakte von der Kommission verabschiedet, vgl. Kommission der EG $=$ XXIII. Gesamtbericht $=40$.

38 Vgl. Rat der EG = Spielzeug-Richtlinie 1988=1ff.

39 Vgl. Kommission der EG = Rasenmäher-Richtlinie $1987=22 \mathrm{ff}$.

$40 \mathrm{Vgl}$. Niskanen $=$ Bureaucracy $=25$.

41 Vgl. dazu insbesondere Hirn, Thelen $=$ Einheit, Vielfalt, Einfalt $=15 \mathrm{f}$. 


\section{c) Die Technologiepolitik}

Positive Spillover-Effekte und die Ausnutzung von economies of scale werden schließlich auch für die Rechtfertigung der Technologiepolitik auf Gemeinschaftsebene ins Feld geführt. Die Internationalisierung der Wissenschaft nimmt schon lange keine Rücksicht auf nationale Grenzen mehr. Eine Bündelung der Kräfte Europas im Technologie- und Forschungsbereich erhöht die Wirksamkeit und verhindert Doppelforschungen. Vor allem die Verbreitung der Ergebnisse der Grundlagenforschung und die Förderung der Mobilität der Wissenschaftler lassen positive externe Effekte erwarten. ${ }^{\mathbf{4 2}}$ Die Erhöhung der Transparenz auf dem Gebiet der Forschung und eine arbeitsteilige Kooperation können von Vorteil sein. Dennoch ist zu beachten, daß sich eine zu starke Koordinierung der Forschung auch hemmend auswirken kann, nämlich dann,
"wenn die Bearbeiter bestimmter Aufgaben keinem Wett- bewerbsdruck mehr unterliegen, weil sie wissen, daß außer ihnen sonst niemand mehr in Europa am selben Thema arbeitet. Er- klärungen dafür, warum man denn mit seiner durch EG-weite Ab- stimmung zugewiesenen. Teilaufgabe nicht rechtzeitig fertig geworden ist, finden sich immer. Der Wettbewerb um die mit der ersten Veröffentlichung zu einem neuen Sachgebiet verbundenen Reputation ist in der öffentlichen Forschung mangels überzeu- gender anderer Anreizsysteme besonders wichtig."

Im übrigen verhindert ein gewisser Wettbewerb das Risiko des Fehlschlags, wenn gleichzeitig an alternativen Lösungen gearbeitet wird. ${ }^{\mathbf{4 4}}$ Die derzeit starke Tendenz zur Verlagerung von technologiepolitischen Kompetenzen auf die Gemeinschaft zeigt, daß diese Argumente nicht genügend berücksichtigt werden. Die Zentralisierung forschungspolitischer Kompetenzen geht weit über das hinaus, was allokationspolitisch sinnvoll wäre.

Allerdings zielt die europäische Technologiepolitik nicht nur auf die Förderung der Spitzenforschung ab, sondern durch die Beteiligung weniger entwickelter Mitgliedstaaten an den Forschungsprojekten soll auch ein kostenloser Technologietransfer in diese Mitgliedstaaten stattfinden. ${ }^{45}$ Damit soll ihnen beim Aufholen ihres technologischen Rückstandes geholfen werden. Für die weiter ent-

42 Vgl. Starbatty, Vetterlein $=$ Technologiepolitik $=38$.

43 Starbatty, Vetterlein $=$ Technologiepolitik $=134$.

44 Vgl. Starbatty, Vetterlein $=$ Technologiepolitik $=38$.

45 Vgl. dazu im folgenden Starbatty, Vetterlein =Technologische Aufgabenteilung= $98 \mathrm{ff}$. 
wickelten Staaten - wie die Bundesrepublik Deutschland -, die ausgeprägte Forschungsbürokratien besitzen, bedeutet eine entsprechende Kompetenzverlagerung auf die Gemeinschaftsebene eine Verlangsamung der Aktivitäten und eine breitere Streuung bisher national eingesetzter Mittel. Sie versuchen daher, möglichst große Teile der Technologiepolitik unter nationaler Kontrolle zu halten. Bei den Projekten auf Gemeinschaftsebene plädieren sie für eine strikte Trennung zwischen ökonomischen und integrationspolitischen (d. h. in der Regel verteilungspolitischen) Zielen und für deren Umsetzung in unterschiedlichen Programmen.

In den weniger entwickelten Mitgliedstaaten, die keine ausgebildete Forschungsbürokratie besitzen und denen auch die nötigen Mittel für die Technologiepolitik fehlen, ist dagegen die Bereitschaft groß, die weder ausgeübten noch finanzierbaren Kompetenzen in diesem Politikbereich nach Brüssel zu verlagern, um dann Forderungen an die dortige Bürokratie zu stellen. Da diese Mitgliedstaaten bei Abstimmungen im Rat großes Gewicht besitzen, versucht die Kommission von vornherein einerseits die Spitzenforschung und andererseits die Entwicklung benachteiligter Gebiete in den Programmen zu berücksichtigen. Die Technologiepolitik der Europäischen Gemeinschaften steht damit in einem Spannungsverhältnis zwischen allokativen und distributiven Zielen.

Diese Vermischung von Zielen ist jedoch problematisch. Auf der einen Seite wird eine effiziente Technologiepolitik um so mehr gefährdet, je stärker der Einfluß der verteilungspolitischen Zielsetzungen wird. Auf der anderen Seite ist grundsätzlich zu fragen, ob mit der Technologiepolitik überhaupt verteilungspolitische Zielsetzungen verfolgt werden sollten. Gerade für Mitgliedstaaten, die in ihren Wirtschaftsstrukturen erhebliche Differenzen aufweisen und die unterschiedliche komparative Kostenvorteile besitzen, scheint eine gemeinsame Forschung nicht (von vornherein) sinnvoll. Der Technologietransfer zeigt nur dann Wirkung, wenn er zur Wirtschaftsstruktur paßt, d. h. wenn er von der Wirtschaft genutzt werden kann. Die gemeinsame Forschungspolitik kann nur bedingt dazu beitragen, das wirtschaftliche Gefälle auszugleichen. Der wirtschaftliche Rückstand bestimmter Mitgliedstaaten muß mit anderen Maßnahmen aufgeholt werden. 
Die Vermischung allokativer und distributiver Zielsetzungen wird bei zwei der bedeutendsten Politikbereiche der Gemeinschaft, der Regionalpolitik und der Agrarpolitik, noch deutlicher.

\section{d) Die Regionalpolitik}

Die Regionalpolitik soll einerseits interregionale Wohlstandsunterschiede ausgleichen, andererseits soll sie helfen, die Wachstumspotentiale entwicklungsfähiger Regionen besser zu nutzen. Beide Ziele müssen nicht von vornherein konfliktär sein. Existieren "überagglomerierte und unteragglomerierte" Regionen nebeneinander, kann eine interregionale Ressourcenumverteilung zur Maximierung des gesamtwirtschaftlichen Wachstums (und damit zu einer optimalen Ressourcenallokation) führen. Denn durch die Umverteilung werden die wirtschaftsschwachen Regionen in die Lage versetzt, vorhandene Ressourcen besser zu nutzen, was dann insgesamt zu einer Produktivitätssteigerung und auch zu einer Wohlfahrtssteigerung führt. ${ }^{46}$ Insofern kann es einen gewissen Umverteilungsspielraum geben, "innerhalb dessen sowohl das Wachstum der EG insgesamt erhöht und gleichzeitig die regionalen Disparitäten verringert werden können"47. Die Förderung schwächer entwickelter Regionen kann also durchaus von allgemeinem Interesse sein, wenn sie zumindest mittelbar Spillover-Effekte (z. B. durch eine verbesserte Arbeitsteilung oder eine Stimulierung des Wachstums) hervorbringt.

Dies gilt allerdings nur innerhalb eines gewissen Spielraums. Wird das Ziel der Umverteilung zu stark betont, besteht die Gefahr, daß die Regionalpolitik implizit mögliches Wachstum in den weiterentwickelten Regionen bremst, auch wenn sie vorwiegend darauf ausgerichtet ist, das Wachstum der benachteiligten Regionen zu beschleunigen. Es ist jedoch ein Trugschluß zu glauben, durch ein Abbremsen der Wachstumsprozesse in den höher entwickelten Staaten wandere das Kapital in die weniger entwickelten. Das Kapital wandert vielmehr in die wachstumsträchtigeren Industrien außerhalb der Gemeinschaft. ${ }^{48}$ Bestehende Wettbewerbsvorteile der entwickelteren Staaten sollten nicht beseitigt, sondern genutzt werden, "denn damit steigt das Einkommen

46 Vgl. dazu Biehl $=$ Europäische Regionalpolitik $=132 \mathrm{f}$.

47 Biehl =Europäische Regionalpolitik $=155$.

48 Vgl. dazu Noé $=$ Regionalpolitische Versuche $=15$. 
der Gesamtwirtschaft und entsprechend auch der Teil, der für etwaige Umverteilung zur Verfügung gestellt werden könnte" 49.

Außerdem darf nicht übersehen werden, daß die regionalen Disparitäten auch sehr stark durch die nationale Wirtschaftspolitik beeinflußt werden. Die Regionalpolitik der Gemeinschaft sollte nicht dazu dienen, die Fehler der nationalen Politik zu korrigieren. Damit wird deutlich, daß die europäische Regionalpolitik von vornherein nur subsidiär tätig werden kann: sie kann Einzelstaaten nur helfen, ihre regionalen Disparitäten abzubauen, wenn auch die nationale Politik darauf zugeschnitten ist. Ist diese Voraussetzung nicht gegeben, dann nützt es auch nichts, den Europäischen Fonds für regionale Entwicklung mit dem Argument, die Wirksamkeit sei ausgeblieben, weil nicht genügend Mittel zur Verfügung stehen, immer weiter aufzublähen. ${ }^{\mathbf{5 0}}$

Dafür, daß der Europäische Fonds für regionale Entwicklung nur unterstützend tätig wird und die Planung und Durchführung von regionalpolitischen Maßnahmen auf dezentraler Ebene erfolgen soll, sprechen auch folgende Argumente:

"Die Politikgestaltung rückt räumlich näher an die Probleme heran, regionalspezifische Engpaßfaktoren könnten eher und präziser ausgemacht, auf neue Entwicklungen und Erfahrungen könnte flexibler reagiert werden, und vielleicht ließen sich in manchen Fällen auch bürokratische Hemmnisse vermeiden. Die dezentral betriebene Suche nach Möglichkeiten, Entwicklungen anzustoßen, würde vermutlich auch zu einem größeren Erfahrungsschatz hinsichtlich der Tauglichkeit und der Kosten verschiedener Förderkonzepte und Instrumente führen. "

Außerdem kann man einen verstärkten interregionalen Wettbewerb erwarten. Ein Wettbewerb in der Qualität des Förderinstrumentariums umgeht das Risiko eines einheitlichen, möglicherweise falsch angelegten Fördersystems und intensiviert die Suche nach Entwicklungsanreizen, die spezifischen Problemen besser gerecht werden. $\mathbf{5 2}$

Es gibt zahlreiche Beispiele dafür, daß regionale Gegebenheiten von der Kommission nicht ausreichend berücksichtigt werden. So werden in Irland als häu-

49 Krieger-Boden $=$ Regionalpolitik der $E G=95$.

50 Vgl. dazu Noé $=$ Regionalpolitische Versuche $=15 \mathrm{f}$.

51 Sachverständigenrat zur Begutachtung der gesamtwirtschaftlichen Entwicklung =Jahresgutachten 1984/85= Ziff. 416.

52 Vgl. Fleckenstein $=$ Regionale Wirtschaftsförderung $=178$. 
figstes Problem im Industriesektor die hohen Energiekosten genannt. Im Rahmen des Europäischen Fonds für regionale Entwicklung wird jedoch der Entwicklung von Wasserkraftwerken und der verstärkten Nutzung von Torf, d. h. der kostenintensivsten Form der Energieerzeugung, Vorrang eingeräumt. In Frankreich wurden für die Auvergne Maßnahmen des Europäischen Fonds für regionale Entwicklung im Bereich der Wärmekraft vorgesehen. Da sie jedoch nicht an den Bedarf angepaßt waren, fanden sie kaum Anwendung. ${ }^{53}$ Im übrigen scheint die Kommission selbst kein großes Interesse an einer Überprüfung der Wirksamkeit der europäischen Regionalpolitik zu haben. ${ }^{\mathbf{5 4}}$

Insgesamt ist festzuhalten, daß die europäische Regionalpolitik weit über das hinausgeht, was ökonomisch geboten ist, und daß es zahlreiche Gründe für eine stärkere Dezentralisierung der Regionalpolitik gibt.

Die Verteilung der Kompetenzen bei der Regionalpolitik ist erst dann vollständig überprüft, wenn auch der Einfluß der Beihilfeaufsicht der Gemeinschaft auf die nationale Regionalpolitik berücksichtigt wird.

Die nationalen Maßnahmen zur Strukturförderung unterliegen insofern nicht mehr nur den nationalen Ebenen, als sie der Beihilfekontrolle der Kommission nach Art. 92ff. EWGV unterworfen werden. Diese Kontrolle ist wettbewerbspolitisch motiviert: Die Beihilfen dürfen den Wettbewerb nicht verfälschen. Grundsätzlich besteht eine Meldepflicht für alle staatlichen Beihilfen, auch für die der Länder. ${ }^{55}$ Nach der Meldung werden die Beihilfen von der Kommission geprüft. Kommt sie zu einem positiven Ergebnis oder macht sie innerhalb einer Frist von zwei Monaten keine Einwände, kann der Mitgliedstaat die Beihilfe durchführen. Andernfalls eröffnet die Kommission das sog. "förmliche Verfahren". In diesem Fall darf der Mitgliedstaat die Beihilfe nicht vor einer

53 Vgl. Europäischer Rechnungshof = Jahresbericht $1989=134$.

54 "In der Praxis hat die Kommission bis zum heutigen Tage keine Arbeitsmethodik zur Bewertung der Vorhaben oder Programme entwickelt. Darüber hinaus sind die allgemein festgestellte mangelnde Präzisierung der Ziele und die unzureichende Erläuterung der Mechanismen dieser Ziele nicht dazu geeignet, mögliche Bewertungen zu erleichtern. Ferner waren die Dienststellen der Kommission kaum darum bemüht, die notwendigen Informationen ... zu erhalten. Im Jahr 1989 hatten drei Mitgliedstaaten noch nicht die statistischen Übersichten für das Jahr 1984 übermittelt; die neuesten jährlichen statistischen Informationen, über die die Kommission verfügt, stammen aus dem Jahr 1987 und betreffen nur einen einzigen Mitgliedstaat. " Europäischer Rechnungshof =Jahresbericht $1989=135$.

Vgl. dazu im folgenden Matthies $=$ Subventionsrecht $=452$. 
abschließenden Entscheidung der Kommission gewähren (Sperrwirkung); er hat außerdem die abschließende Entscheidung zu respektieren.

Bei der Beurteilung, welche Beihilfen mit dem Gemeinsamen Markt als vereinbar angesehen werden können und welche nicht, ist zwischen Art. 92 Abs. 3 Buchstabe a und Buchstabe c EWGV zu unterscheiden. Nach Art. 92 Abs. 3 Buchstabe a können Beihilfen zur Förderung der wirtschaftlichen Entwicklung von Gebieten, in denen die Lebenshaltung außergewöhnlich niedrig ist oder eine erhebliche Unterbeschäftigung herrscht, als mit dem Gemeinsamen Markt vereinbar angesehen werden. Der Europäische Gerichtshof hat in seinem Urteil zur Entscheidung der Kommission, die vom Land Nordrhein-Westfalen vorgesehenen Beihilfen in den Arbeitsmarktregionen Borken-Bocholt und Siegen für mit dem Gemeinsamen Markt unvereinbar zu erklären, festgestellt, daß sich die Vorschriften des Art. 92 Abs. 3 Buchstabe a EWGV auf Gebiete beziehen, in denen die wirtschaftliche Lage im Vergleich zur gesamten $\mathrm{Ge}$ meinschaft äußerst ungünstig ist. ${ }^{56}$ Für die Beurteilung der Förderungswürdigkeit wird hier also das Gemeinschaftsniveau als Bezugsgröße herangezogen. Das bedeutet für die reicheren Mitgliedstaaten, daß ihre benachteiligten Gebiete sehr stark vom nationalen Durchschnitt abweichen müssen, um überhaupt als förderungswürdig angesehen zu werden. Für die Bundesrepublik Deutschland - in der Gestalt vor dem 3.10.1990 - hat die Kommission mehrfach festgestellt, daß ihre wirtschaftliche und soziale Lage weder im Ganzen noch in Teilgebieten die Anwendung von Art. 92 Abs. 3 Buchstabe a rechtfertigt. ${ }^{57}$ Die regionalen Beihilfen der Bundesrepublik Deutschland werden von der Kommission also nur nach Art. 92 Abs. 3 Buchstabe b genehmigt.

Nach diesem Artikel können sektorale und regionale Beihilfen zulässig sein, wenn sie die Handelsbedingungen nicht in einer Weise verändern, die dem gemeinsamen Interesse zuwiderlaufen. Die Kommission ist dabei der Ansicht, daß die von einer Regionalbeihilfe ausgehende Veränderung der Handelsbedingungen nur dann als dem gemeinsamen Interesse nicht zuwiderlaufend angesehen werden kann, wenn sich feststellen läßt,

- daß die betroffenen Regionen im Gemeinschaftsrahmen unter erheblichen Schwierigkeiten $\mathrm{zu}$ leiden haben,

56 Vgl. EuGH Rs. 248/84, zitiert nach Rengeling $=$ Subventionsrecht $=466$.

57 Vgl. Kommission der EG = Regionale Beihilfen $1986=22$. 


\section{Mangelnde Berücksichtigung der Vorteile eines föderativen Aufbaus}

- daß die Marktkräfte ohne die Beihilfen diese Schwierigkeiten nicht beseitigen würden,

- daß die Höhe der Beihilfe diesen Schwierigkeiten angepaßt ist und

- daß die Beihilfevergabe nicht in bestimmten Wirtschaftszweigen den Wettbewerb im Übermaß verfälscht. 58

Zur Überprüfung nationaler Beihilfen legte die Kommission konkrete Schwellenwerte, ausgedrückt durch Arbeitslosigkeit und Bruttowertschöpfung pro Einwohner, fest. Diese Schwellenwerte werden aus der Relation des jeweiligen Durchschnitts des Einzelstaates zum Gemeinschaftsdurchschnitt abgeleitet. In der Bundesrepublik Deutschland sind Gebiete dann förderungwürdig, wenn ihre Bruttowertschöpfung pro Einwohner $74 \%$ des Bundesdurchschnitts unterschreitet oder ihre Arbeitslosenquote im Fünfjahresdurchschnitt mehr als $143 \%$ des Bundesdurchschnitts beträgt. 59

Da die Schwellenwerte am Gemeinschaftsniveau anknüpfen, ziehen sie die Förderungswürdigkeit um so enger, je günstiger die allgemeine Wirtschaftslage des betreffenden Mitgliedstaates ist. ${ }^{60}$ Bei der Beurteilung der Beihilfen wird damit jedoch das Wettbewerbsziel der Gemeinschaft vom Ausgleichsziel überlagert: Die eigentlich strikt wettbewerbsbezogenen Vertragsnormen der Beihilfeaufsicht werden dazu verwendet, ein auf Regionen bezogenes Konvergenzziel der Kommission durchzusetzen. ${ }^{61}$ Die Tatsache, daß nationale Beihilfen, die sich an nationalen Maßstäben orientieren, anhand des gemeinschaftlichen Ausgleichsziel beurteilt werden, das an Durchschnittswerten der Gemeinschaft ansetzt, führt unweigerlich zu Friktionen. Zielt ein Mitgliedstaat mit weit über dem Gesamtdurchschnitt liegender Wirtschaftsentwicklung auf einen Ausgleich wirtschaftlicher Ungleichgewichte verschiedener innerstaatlicher Regionen, so kann dies aus Sicht der Gemeinschaft als weitere Verschärfung der regionalen Unterschiede in der Gemeinschaft und damit als unerwünschte Entwicklung betrachtet werden. "Daß eine weniger entwickelte Region eines Mitgliedstaates mehr entwickelt sein kann als eine mehr entwickelte Region ei-

$58 \mathrm{Vgl}$. Kommission der EG = Regionale Beihilfen 1986= 22 .

59 Vgl. Kommission der EG $=$ Beihilfen der Freien und Hansestadt Hamburg $=6$.

60 Vgl. Püttner, Spannowsky = Verhältnis europäischer zu deutscher Regionalpolitik= 198.

61 Vgl. dazu auch Zimmermann =EG-Begrenzungen für deutsche Regionalpolitik= 97. 
nes anderen Mitgliedstaates, ergibt sich aus dem wirtschaftspolitischen Entwicklungsgefälle der Mitgliedstaaten. "62

Eine Orientierung am Wettbewerbsziel verlangt, daß Beihilfen mit vergleichbaren Wirkungen auf den Wettbewerb in allen Mitgliedstaaten gleich behandelt werden. Die Festlegung der Förderwürdigkeit anhand von Schwellenwerten kann dies verhindern. Während in Gebieten, die von den Europäischen Gemeinschaften als nicht förderungswürdig angesehen werden, auch die Förderung von mittleren Unternehmen des Handwerksbereiches, die nur geringe Handelsbeeinträchtigungen verursachen, unzulässig ist, können in Gebieten, die als förderungswürdig anerkannt werden, einzelne Beihilfen (z. B. für ein Großunternehmen) zugelassen werden. Dieser Einwand gegen die Prüfmethode der Kommission darf nicht dahingehend mißverstanden werden, daß generelle Beihilfen in den Mitgliedstaaten ohne Einschränkung befürwortet werden. Vielmehr geht es darum, daß die Beihilfe unter dem Wettbewerbsziel und nicht unter dem Ausgleichsziel zu beurteilen ist. Widerspricht sie dem Wettbewerbsziel, ist sie abzulehnen. Die pauschale Festlegung der Förderungswürdigkeit durch die Schwellenwerte erlaubt dagegen nicht immer die Differenzierung nach unterschiedlichen Beihilfen - mit unterschiedlichen Wettbewerbswirkungen. ${ }^{63}$

Die Festlegung der Schwellenwerte bedeutet für die reichen Mitgliedstaaten wie die Bundesrepublik Deutschland eine weit größere Einschränkung des Handlungsspielraumes als für die ärmeren Mitgliedstaaten. Wenn die Kommission für die reicheren Mitgliedstaaten restriktivere Grenzwerte setzt, so daß erst größere Abweichungen vom nationalen Durchschnitt die Anerkennung als Fördergebiet rechtfertigen, stuft sie das Ausgleichsanliegen der reicheren Staaten teilweise als nicht anerkennenswert ein. Dadurch wird den Mitgliedstaaten vorgegeben, welche Lebensverhältnisse noch als einheitlich anzusehen sind und welche nicht. ${ }^{64}$

Die Beihilfenaufsicht sollte sich am Wettbewerbsziel orientieren. Dieses Ziel muß klar vom Ausgleichsziel getrennt werden. Die Beihilfen sollten entspre-

62 Vgl. Püttner, Spannowsky = Verhältnis europäischer zu deutscher Regionalpolitik= 140.

63 Vgl. Püttner, Spannowsky = Verhältnis europäischer zu deutscher Regionalpolitik= $212 \mathrm{ff}$. und Bundesministerium für Wirtschaft $=$ Regionalpolitik $=34$.

64 Vgl. Püttner, Spannowsky = Verhältnis europäischer zu deutscher Regionalpolitik= 230. 
chend ihren Wettbewerbseffekten beurteilt werden und nicht danach, ob sie in einem reichen oder einem armen Mitgliedstaat gewährt werden. Nur so kann auch den reicheren Mitgliedstaaten ein angemessener Spielraum zur selbständigen Bekämpfung regionaler Strukturprobleme eingeräumt werden. ${ }^{65}$ Das Ausgleichsziel sollte im Rahmen des Strukturfonds verfolgt werden. Dazu ist es notwenig, die Mittel dieses Fonds auf die schwächsten Regionen der Gemeinschaft zu konzentrieren.

\section{e) Die Agrarpolitik}

Eine Agrarpolitik auf Gemeinschaftsebene kann grundsätzlich mit gewissen Spillover-Effekten begründet werden. Ein echter gemeinsamer Agrarmarkt, bei dem jeder Mitgliedstaat seine Standortvorteile im Wettbewerb nutzen kann, hätte allen Mitgliedstaaten wirtschaftliche Vorteile gebracht. Auch das Argument der für alle Mitgliedstaaten gesicherten Versorgung läßt sich hier anführen. ${ }^{66}$ Tatsächlich war die Agrarpolitik jedoch von Anfang an gesellschaftspolitisch motiviert. Sie wurde bereits sehr früh in die wirtschaftliche Integration einbezogen, obwohl große Unterschiede in der Struktur der Landwirtschaft und der Agrarförderung und damit auch große Interessengegensätze zwischen den einzelnen Mitgliedstaaten bestanden. ${ }^{67}$ Auf eine gemeinsame Agrarpolitik drängten vor allem jene Mitgliedstaaten, die im Industriebereich unterproportionale Integrationsvorteile oder sogar Integrationsnachteile befürchteten, und die gleichzeitig ein großes landwirtschaftliches Potential zur Verfügung hatten. Hinter dem Vorgang steckt letztlich das oben beschriebene Problem des "package-deals": um Mitgliedstaaten zur Zustimmung in einem bestimmten Bereich zu bewegen, muß man ihnen auf anderen Gebieten entgegenkommen. Die unterschiedlichen Interessen der Mitgliedstaaten blieben für die Agrarpolitik immer bedeutsam. Dies zeigte sich daran, daß immer mehr Produkte in das System der Preisgarantie einbezogen werden mußten, um den Strukturen der einzelnen Mitgliedstaaten gerecht zu werden.

Auch bei der Agrarpolitik wurden allokative und verteilungspolitische Zielsetzungen verfolgt, wobei das verteilungspolitische Ziel im Laufe der Zeit eben-

65 Vgl. zu dieser Forderung auch Planungsausschuß für regionale Wirtschaftsstruktur $=16$. Rahmenplan $=22$.

66 Vgl. dazu Kommission der EG = MacDougall-Bericht $=394$.

67 Vgl. Thoroe =Europäische Agrarpolitik= 86 . 
falls sehr stark in den Vordergrund rückte. Die Garantiepreise, die ursprünglich dazu dienen sollten, die Landwirte vor erratischen Schwankungen der Marktpreise zu schützen, entfernten sich aufgrund der einkommenspolitischen Zielsetzungen immer mehr vom Gleichgewichtspreis, der einen Ausgleich von Angebot und Nachfrage bewirkt. Der Preis konnte seine Funktion als Knappheitsindikator nicht erfüllen; die Folge waren Überproduktion (siehe Tabelle 19) und steigende Lagerkosten. Auch wenn in einzelnen Jahren die Lagerbestände bestimmter Produkte zurückgeführt werden konnten, wurde dieses Problem nie grundsätzlich gelöst. ${ }^{68} \mathrm{Da}$ die Garantiepreise über den Weltmarktpreisen lagen, entstanden auch hohe Kosten durch die Exporterstattungen. Die teilweise sehr komplizierten Einzelregelungen der verschiedenen Agrarmarktorganisationen haben außerdem zu einer steigenden Zahl von Betrugsfällen geführt. ${ }^{69}$

Das Ziel eines gemeinsamen Agrarmarktes konnte ebenfalls nicht verwirklicht werden. Der Grenzausgleich im Agrarhandel hat dazu geführt, daß der europäische Agrarmarkt wieder aus so vielen nationalen Teilmärkten besteht, wie die Europäische Gemeinschaft Mitglieder hat. ${ }^{70}$ Die Interventionspreise der Agrarmarktordnungen werden in ECU festgelegt. Wechselkursänderungen haben zur Folge, daß sich das jeweilige Verhältnis der nationalen Währungen zum ECU ändert. Damit ändert sich auch die Umrechnung der Interventionspreise in nationale Währungen: bei Aufwertungen müßten die in nationalen Währungen ausgedrückten Interventionspreise um den Aufwertungssatz sinken, bei Abwertungen müßten sie um den Abwertungssatz steigen. Um diese Effekte der Wechselkursänderungen nicht wirksam werden zu lassen, wurde ein innergemeinschaftlicher Grenzausgleich für den Handel mit Agrarprodukten eingeführt. Aufwertungsländer, die eigentlich für Zusatzeinfuhren in Betracht kommen, dürfen eine Art Einfuhrzoll erheben, der es ihnen gestattet, die nationalen Interventionspreise auf dem alten Stand zu halten. Abwertungsländer andererseits legen ihrer Landwirtschaft eine Art von Exportzöllen auf.

68 So konnten zwar der "Butterberg" sowie die Lagerbestände an Rindfleisch und Getreide in den Jahren 1988 und 1989 etwas verringert werden, doch stiegen die Bestände 1990 wieder deutlich an. Vgl. =Agrarbericht $1991=85 \mathrm{ff}$.

69 Vgl. dazu u. a. Hirn = Agrarbetrügereien $=56 \mathrm{ff}$. und o. V. $=$ Betrügereien $=18$.

70 Vgl. Reding = Problematik eines Finanzausgleichs $=214$. Daran hat auch die Verordnung über das System des automatischen Abbaus der negativen Währungsausgleichsbeträge bisher nichts geändert. Kommission der EG = Negative Währungsausgleichsbeträge $=16 \mathrm{ff}$. 
Tabelle 19: Der Selbstversorgungsgrad1) bei ausgewählten landwirtschaftlichen Erzeugnissen in den Mitgliedstaaten 1989/902)

\begin{tabular}{|c|c|c|c|c|c|c|}
\hline $\begin{array}{l}\text { Mitglied- } \\
\text { staat }\end{array}$ & Getreide $^{3)}$ & Zucker ${ }^{3)}$ & $\begin{array}{l}\text { Rind-und } \\
\text { Kalbfleisch }\end{array}$ & $\begin{array}{l}\text { Schweine- } \\
\text { fleisch }\end{array}$ & Butter & Milchpulver \\
\hline B & 64 & 232 & 150 & 172 & 105 & 258 \\
\hline DK & 141 & 248 & 208 & 351 & 177 & 87 \\
\hline $\mathrm{D}$ & 101 & 132 & 88 & 113 & 85 & 549 \\
\hline GRL & 99 & 129 & 29 & 63 & 50 & - \\
\hline E & 95 & 94 & 98 & 96 & 174 & 150 \\
\hline $\mathrm{F}$ & 243 & 213 & 108 & 85 & 110 & 142 \\
\hline IRL & 103 & 179 & 716 & 117 & 927 & 1400 \\
\hline I & 77111 & & 56 & 68 & 59 & - \\
\hline NL & 29 & 209 & 158 & 272 & 453 & 51 \\
\hline P & 63 & 1 & 83 & 92 & 99 & 133 \\
\hline UK & 115 & 54 & 87 & 68 & 100 & 100 \\
\hline EG(12) & 121 & 130 & 101 & 102 & 112 & $161^{4)}$ \\
\hline \multicolumn{7}{|c|}{$\begin{array}{l}\text { 1) Inlandserzeugung in \% des Gesamtverbrauchs an Nahrungs- und Futtermitteln einschließlich Verbrauch } \\
\text { infolge von Sondermaßnahmen. } \\
\text { 2) Tierische Produkte Kalenderjahr } 1989 . \\
\text { 3) Vorläufig. } \\
\text { 4) Geschätzt. }\end{array}$} \\
\hline
\end{tabular}

Quelle: $=$ Agrarbericht $1991=82$. 
Diese Praxis unterbindet den Preiswettbewerb innerhalb der Gemeinschaft. ${ }^{71}$ Solange es noch Wechselkursänderungen in der Europäischen Gemeinschaft gibt, bleibt der Grenzausgleich relevant. Mit einem Gemeinsamen Markt ist diese Praxis nicht vereinbar, nicht zuletzt deshalb, weil sie Grenzkontrollen erforderlich macht. ${ }^{72}$

Die allokativen Nachteile des Agrarmarktsystems wurden mit dem Hinweis auf das Ziel der Einkommenssicherung der Landwirte gerechtfertigt. Doch auch dieses Ziel wurde nur sehr bedingt erreicht: ${ }^{73}$ die Kaufkraft der in der Landwirtschaft beschäftigten Personen ist im Zeitraum 1975-1989 nur sehr geringfügig gestiegen. Eine Einkommensstützung, die fast ausschließlich in Form von Preisgarantien erfolgt, kommt außerdem vor allem großen Betrieben mit hohem Intensivierungsgrad zugute, so daß sich innerhalb des Agrarsektors unerwünschte Verteilungseffekte ergeben. Dies hat dazu geführt, daß im Laufe der Zeit eine Reihe von direkten Einkommensbeihilfen für kleine Betriebe eingeführt werden mußte.

Reformvorschläge beinhalten häufig die Idee, die Einkommenssicherung grundsätzlich mit anderen Instrumenten als der Agrarmarktpolitik zu betreiben. "Direkte Einkommensübertragungen anstelle einer expansiven Preispolitik erscheinen hierfür ein gangbarer Weg."74 Inzwischen werden auch bei der Kommission Überlegungen zu Preissenkungen und kompensierenden direkten Preishilfen angestellt. Ihre Vorschläge für die Agrarpreise 1991/92 sehen neben direkten Preiskürzungen, z. B. bei Zucker um 5\% oder bei Tabak um $10 \%$, auch indirekte Preissenkungen vor. Bei Rindfleisch wurde der Prozentsatz, ab dem staatliche Stellen Agrarprodukte aufkaufen, von $88 \%$ auf $80 \%$ herabgesetzt, was in der Praxis einer Preissenkung entspricht. ${ }^{75}$ Die als Ausgleich für die Preiskürzungen vorgesehenen Einkommensbeihilfen sollen teilweise produktspezifisch, je nachdem wie stark die Landwirte von Verschärfungen der Agrarmarktordnungen betroffen sind $^{\mathbf{7 6}}$, sowie teilweise speziell für

71 Vgl. Willgerodt $=$ Agrarpolitik der $\mathrm{EG}=108$.

72 Vgl. Kommission der EG $=$ Vollendung des Binnenmarktes $=12$.

73 Vgl. im folgenden Kommission der EG $=$ Reform der Agrarpolitik $=7$.

74 Thoroe =Europäische Agrarpolitik $=118$.

75 Vgl. Kommission =Preisvorschläge 1991/92 $=62$.

76 Wie z. B. bei der Hartweizenbeihilfe. Vgl. Kommission =Preisvorschläge 1991/92= 8. 


\section{Mangelnde Berücksichtigung der Vorteile eines föderativen Aufbaus}

kleine Erzeuger ${ }^{77}$ gewährt werden. Die Vorschläge der Kommission stellen nur die ersten Schritte zu einer veränderten Agrarmarktpolitik dar. Eine grundsätzliche Reform, die dem Preis wieder seine Lenkungsfunktion zurückgibt, ist nicht in Sicht; die Preise werden auch weiterhin administrativ festgelegt.

Grundsätzlich stellt sich die Frage, ob es überhaupt sinnvoll ist, direkte Einkommensübertragungen auf Gemeinschaftsebene zu installieren, denn sie könnten wohl kaum an einem gemeinsamen Referenzsystem ausgerichtet werden. Die Angemessenheit der Lebenshaltung, wie sie im Vertrag zur Gründung der Europäischen Wirtschaftsgemeinschaft für die landwirtschaftliche Bevölkerung angestrebt wird, läßt sich sinnvoll nur im Verhältnis zur Einkommenslage und Einkommensentwicklung des sozialen Umfeldes definieren. Wegen der starken Einkommensunterschiede innerhalb der Europäischen Gemeinschaften wäre deshalb eine nationale und auch regionale Differenzierung des Zielsystems für die landwirtschaftliche Einkommenspolitik erforderlich. ${ }^{78} \mathrm{Da}$ eine Einigung über solche Differenzierungen im Rahmen der gemeinsamen Agrarpolitik nur schwer zu erreichen sein wird, ist es sinnvoll, direkte Einkommensübertragungen in die Kompetenz der Mitgliedstaaten zurückzuverlagern.

1989 hat die Kommission eine Verordnung verabschiedet, nach der die Mitgliedstaaten die Einkommenseinbußen, die im Zuge von Preissenkungen oder der Einführung von Stabilisatoren auftreten, durch direkte, produktionsneutrale, auf höchstens fünf Jahre begrenzte und degressiv gestaffelte Beihilfen ausgleichen können. ${ }^{79}$ An diesen Beihilfen beteiligen sich die Europäischen Gemeinschaften je nach dem wirtschaftlichen Entwicklungsstand der Regionen finanziell in unterschiedlicher Höhe. In der Bundesrepublik Deutschland sind die Länder für die Durchführung und auch für die Finanzierung von Einkommensbeihilfen zuständig. Bisher beabsichtigen Baden-Württemberg und Rheinland-Pfalz, entsprechende Einkommensbeihilfen einzuführen. ${ }^{80}$

77 Vgl. Kommission $=$ Preisvorschläge $1991 / 92=12$.

78 Vgl. Thoroe =Europäische Agrarpolitik $=120$.

79 Vgl. Rat der EG =Einkommensbeihilfen $1989=8 \mathrm{ff}$.

80 Vgl. =Agrarbericht $1991=101$. 


\section{f) Stabilisierungspolitik}

Die Kompetenzverteilung im Rahmen der Stabilisierungspolitik wird bestimmt durch die Entwicklung der Gemeinschaft zu einer Wirtschafts- und Währungsunion, die nach den Plänen der Kommission in drei Phasen verwirklicht werden soll. ${ }^{81}$ Seit dem 1.7.1990 befindet sich die Gemeinschaft in der ersten Phase, bei der die geldpolitischen Kompetenzen noch bei den nationalen Zentralbanken liegen und die Konvergenz der Wirtschaftspolitik der Mitgliedstaaten verstärkt werden soll. Über eine zweite Stufe, in der neben den nationalen Zentralbanken auch eine Europäische Zentralbank geschaffen werden soll, die geldpolitischen Kompetenzen jedoch weiterhin auf nationaler Ebene bleiben, soll schließlich in einer dritten Stufe die geldpolitische Verantwortung vollständig auf die Europäische Zentralbank übergehen und eine einheitliche Währung geschaffen werden. Es ist hier nicht der Raum, um detailliert auf die Probleme der Schaffung einer Wirtschafts- und Währungsunion einzugehen. Es muß an dieser Stelle genügen, einige wesentliche Aspekte aufzuzeigen, die die stabilitätspolitische Aufgabenverteilung zwischen den Europäischen Gemeinschaften und den Mitgliedstaaten betreffen. Dabei kann die geldpolitische Kompetenzverteilung nicht ausgeklammert werden, denn die finanzpolitische Aufgabenverteilung kann nicht völlig unabhängig von der Verteilung der geldpolitischen Kompetenzen betrachtet werden.

Je enger die Märkte für Güter, Dienstleistungen und Kapital verflochten sind, desto eher ist die Verwirklichung eines einheitlichen Währungsraums sinnvoll. Durch ihn ergeben sich Vorteile in Form von geringeren Informations- und Transaktionskosten der Marktteilnehmer. Bei einer ungenügenden wirtschaftlichen Integration stehen diesen Vorteilen jedoch beträchtliche Risiken gegenüber. ${ }^{82}$ Die Wirtschaftsbeziehungen zwischen den zwölf Mitgliedstaaten sind noch immer sehr unterschiedlich. Auch wenn die Märkte im Zentrum der Europäischen Gemeinschaften einen hohen Integrationsgrad aufweisen, so hat doch mit manchen Gebieten der Peripherie der Prozeß der wirtschaftlichen Verflechtung erst begonnen. Dem heutigen Integrationsstand ist eine einheitliche Währung noch nicht angemessen: Noch gibt es Kapitalmarktbeschränkun-

81 Vgl. Kommission der EG = Delors-Bericht 1989=.

82 Vgl. dazu Sachverständigenrat zur Begutachtung der gesamtwirtschaftlichen Entwicklung = Jahresgutachten 1989/90= Ziff. 397ff. und Neumann =Gemeinsame Aufgaben $=85 \mathrm{ff}$. 
gen, Hindernisse für die Mobilität der Arbeitskräfte und vor allem erhebliche Inflationsdifferenzen. 83

Zwar schien sich bei den Inflationsraten seit dem Beginn der achtziger Jahre bis 1988 eine gewisse Annäherung auf niedrigerem Niveau abzuzeichnen. Es ist jedoch fraglich, ob daraus eindeutig geschlossen werden kann, daß in allen Mitgliedstaaten die Vorstellung, Preisniveaustabilität könne zugunsten weniger Arbeitslosigkeit eingetauscht werden, verschwunden ist. Ein wesentlicher Faktor für die niedrigeren Divergenzen bei den Preissteigerungsraten waren vielmehr Außeneinflüsse, wie z. B. sinkende Rohstoffpreise. ${ }^{84}$ Seit Mitte 1988 hat sich die Inflation wieder beschleunigt, allerdings in den einzelnen Mitgliedstaaten unterschiedlich stark (siehe Tabelle 20).

Eine Verlagerung der geldpolitischen Kompetenzen auf die Gemeinschaftsebene birgt für die Bundesrepublik Deutschland vor allem das Risiko geringerer Geldwertstabilität. Es ist zu befürchten, daß das im Europäischen Währungssystem durch die "Ankerfunktion" der D-Mark erzielte Niveau an Geldwertstabilität nicht beibehalten werden kann. Denn während sich die vorhandenen unterschiedlichen Ansichten über den richtigen geldpolitischen Kurs unter dem von der D-Mark ausgehenden Stabilisierungsdruck nicht voll durchsetzen konnten, wird bei der Übertragung der geldpolitischen Verantwortung auf den Ausschuß der Zentralbankpräsidenten bei Meinungsverschiedenheiten viel eher ein Kompromiß auf "mittlerer Linie" erzielt werden. ${ }^{85}$ Entwürfe für ein Statut einer Europäischen Zentralbank sehen zwar vor, daß sie vor allem der Geldwertstabilität verpflichtet sein soll. Dazu muß sie jedoch institutionell und organisatorisch in die Lage versetzt werden. Vor allem muß sie mit einer in institutioneller, funktioneller und personeller Hinsicht dauerhaft gesicherten Unabhängigkeit ausgestattet sein. ${ }^{86}$ Als Grundsatz scheint der Autonomiestatus inzwischen weithin akzeptiert. Die Ansichten darüber, was unter Autonomie zu verstehen ist, gehen jedoch noch weit auseinander. ${ }^{87}$

83 Vgl. Sachverständigenrat zur Begutachtung der gesamtwirtschaftlichen Entwicklung $=$ Jahresgutachten 1989/90 = Ziff. 398 .

84 Vgl. Scheer, Vogelbusch $=$ Gains and Losses $=9$.

85 Vgl. Sachverständigenrat zur Begutachtung der gesamtwirtschaftlichen Entwicklung $=$ Jahresgutachten 1989/90 = Ziff. 402 .

$86 \mathrm{Vgl}$. Deutsche Bundesbank =Errichtung einer $\mathrm{WWU}=43$.

87 Vgl. Neumann $=$ Gemeinsame Aufgaben $=91$. 
Tabelle 20: Die Inflationsraten in den Mitgliedsländern der Europäischen Gemeinschaften (in \%)

\begin{tabular}{|lrrrrrrrr|}
\hline $\begin{array}{l}\text { Mitglied- } \\
\text { staat }\end{array}$ & 1981 & 1984 & 1985 & 1986 & 1987 & 1988 & 1989 & 1990 \\
\hline B & $+7,6$ & $+6,3$ & $+4,9$ & $+1,3$ & $+1,6$ & $+1,2$ & $+3,1$ & $+3,5$ \\
DK & $+11,7$ & $+6,3$ & $+4,7$ & $+3,6$ & $+4,0$ & $+4,6$ & $+4,8$ & $+2,6$ \\
D & $+6,3$ & $+2,4$ & $+2,0$ & $-0,1$ & $+0,2$ & $+1,3$ & $+2,8$ & $+2,5$ \\
E & $+14,6$ & $+11,3$ & $+8,8$ & $+8,8$ & $+5,3$ & $+4,8$ & $+6,8$ & $+6,7$ \\
F & $+13,4$ & $+7,4$ & $+5,8$ & $+2,7$ & $+3,1$ & $+2,7$ & $+3,6$ & $+3,0$ \\
GRL & $+24,5$ & $+18,5$ & $+19,3$ & $+23,0$ & $+16,4$ & $+13,5$ & $+13,7$ & $+20,0$ \\
IRL & $+20,4$ & $+8,6$ & $+5,4$ & $+3,9$ & $+3,2$ & $+2,1$ & $+4,0$ & $+3,2$ \\
I & $+17,8$ & $+10,8$ & $+9,2$ & $+5,9$ & $+4,7$ & $+5,0$ & $+6,3$ & $+6,2$ \\
LUX & $+8,1$ & $+5,6$ & $+4,1$ & $+0,3$ & $-0,1$ & $+1,4$ & $+3,4$ & $+3,7$ \\
NL & $+6,7$ & $+3,2$ & $+2,3$ & $+0,2$ & $-0,4$ & $+0,8$ & $+1,1$ & $+2,5$ \\
P & $+20,0$ & $+29,3$ & $+19,3$ & $+11,7$ & $+9,4$ & $+9,6$ & $+12,6$ & $+13,4$ \\
UK & $+11,9$ & $+5,0$ & $+6,1$ & $+3,4$ & $+4,1$ & $+4,9$ & $+7,8$ & $+4,8$ \\
\hline
\end{tabular}

Quelle: Statistisches Bundesamt $=$ Ausland $1990=140$ und Bundesministerium der Finanzen $=$ Finanzbericht $1992-296 \mathrm{f}$. 
Funktionelle Unabhängigkeit bedeutet zunächst Unabhängigkeit von Weisungen der nationalen Regierungen und der Gemeinschaftorgane. Geklärt werden muß dabei vor allem die Frage des Verhältnisses zwischen der Europäischen Zentralbank und dem Rat der Wirtschafts- und Finanzminister. Die bisherigen Pläne sehen vor, daß der Rat der Wirtschafts- und Finanzminister für eine weitreichende makroökonomische Koordinierung zuständig sein soll. Da eine solche Koordinierung ohne die Geldpolitik unvollständig ist, ist es nur eine Frage der Zeit, bis versucht wird, auch die Geldpolitik einzubeziehen. Von der Koordination bis zur Vorgabe von Leitlinien ist es dann nur noch ein kleiner Schritt. Die funktionelle Unabhängigkeit der Notenbank beinhaltet darüber hinaus aber auch die Entbindung von jeglicher Verpflichtung zur Unterstützung der Wirtschaftspolitik der Europäischen Gemeinschaft. ${ }^{\mathbf{8 8}}$ Eine solche Unterstützungsklausel, die sich am Bundesbankgesetz orientiert, führt die Geldpolitik in ein Spannungsverhältnis. Dieses ist in Deutschland zwar grundsätzlich ebenso gegeben, jedoch wurde dort der Geldwertstabilität in der Regel der Vorrang eingeräumt, weil die deutsche Bevölkerung diesem Ziel hohen Rang beimißt. In Europa besteht keine so ausgeprägte stabilitätspolitische Tradition wie in Deutschland. Daher ist die Gefahr größer, daß das Spannungsverhältnis zwischen der Sicherung der Geldwertstabilität und der Unterstützung der Wirtschaftspolitik sich zu Lasten ersterer auswirkt. ${ }^{89}$

Die personelle Unabhängigkeit betrifft die Auswahl und die Ernennung der Mitglieder des Zentralbankrates und des Direktoriums der Europäischen Zentralbank. ${ }^{90}$ Wenn die Europäische Zentralbank die volle Kontrolle über alle Instrumente besitzt, die das Geldangebot beeinflussen, ist sie schließlich auch institutionell unabhängig. Wichtig ist hier insbesondere, daß eine direkte Finanzierung von Budgetdefiziten der nationalen Regierungen oder der Europäischen Gemeinschaften durch die Europäische Zentralbank verhindert wird.91

Geldwertstabilität kann schließlich auf Dauer nur erreicht werden, wenn auch die staatliche Finanzpolitik in allen Mitgliedstaaten stabilitätsorientiert geführt wird. ${ }^{92}$ Eine unsolide Finanzpolitik eines Mitgliedsstaates hat auf die gesamte

88 Vgl. dazu Willms $=$ Grundprobleme $=552 \mathrm{f}$.

89 Vgl. Sachverständigenrat zur Begutachtung der gesamtwirtschaftlichen Entwicklung $=$ Jahresgutachten 1990/91 = Ziff. 497.

90 Vgl. Willms $=$ Grundprobleme $=553$.

$91 \mathrm{Vgl}$. Willms $=$ Grundprobleme $=553 \mathrm{f}$.

$92 \mathrm{Vgl}$. Deutsche Bundesbank =Errichtung einer $\mathrm{WWU}=43$. 
Gemeinschaft negative Auswirkungen. Sind die Wechselkurse fest, kann das Schuldnerland leichter auf die Ersparnisse anderer Staaten zugreifen. Da das Wechselkursrisiko entfällt, muß das Schuldnerland nur noch das Bonitätsrisiko tragen und auch hier können sich Sonderbedingungen ergeben, wenn der Markt davon ausgeht, daß das entsprechende Mitgliedsland im Ernstfall von den anderen Mitgliedstaaten nicht sich selbst überlassen, sondern gestützt wird. Insofern kann sich der Schuldnerstaat "preiswerter" verschulden. Die Belastung des Kapitalmarkts durch eine unsolide Haushaltspolitik führt zu einem Zinsanstieg, über den die anderen Mitgliedstaaten mitbelastet werden, denn dadurch werden dort Investitionen eingeschränkt. Die Ersparnisse dieser Mitgliedstaaten werden zur Finanzierung eines fremden Defizits beansprucht, ihre stabilitätspolitischen Bemühungen durch eine importierte Inflation erschwert. ${ }^{93}$

Zur Zeit wird in den Mitgliedstaaten noch eine sehr unterschiedliche Budgetpolitik betrieben. Sowohl die laufenden Finanzierungssalden der Budgets als auch die relative Höhe der Staatsschulden weisen gravierende Unterschiede auf (siehe Tabellen 21 und 22).

Im Bericht zur Wirtschafts- und Währungsunion in der Europäischen Gemeinschaft wurde vorgeschlagen, Obergrenzen für die Haushaltsdefizite der einzelnen Mitgliedstaaten und einen mittelfristigen finanzpolitischen Gesamtkurs festzulegen, einschließlich des Umfangs und der Finanzierung des globalen Haushaltssaldos, d. h. sowohl der Haushaltspolitik in den einzelnen Mitgliedstaaten als auch der Haushaltsposition der Gemeinschaft. ${ }^{94}$ Es ist jedoch umstritten, ob Regelungen für die Begrenzung der öffentlichen Haushaltsdefizite notwendig sind. Solchen Überlegungen wird entgegengehalten, daß eine verbindliche Ex-ante-Koordination der Haushaltspolitik der Mitgliedstaaten verfassungspolitisch bedenklich ist, da hierdurch in Befugnisse eingegriffen würde, die zu den Kernbereichen einzelstaatlicher Verantwortlichkeit gehören, und daß die notwendige Konvergenz besser durch Anpassungszwänge über die Marktkräfte hervorgebracht werden soll. 95

93 Vgl. Sachverständigenrat zur Begutachtung der gesamtwirtschaftlichen Entwicklung $=$ Jahresgutachten $1989 / 90=412 \mathrm{ff}$., insbesondere 415 und Issing $=\mathrm{EWS}=3$.

94 Vgl. Kommission der EG = Delors-Bericht $=27$.

95 Vgl. Wissenschaftlicher Beirat beim Bundesministerium für Wirtschaft =Stellungnah$\mathrm{me}=6$ und Scheide, Trapp $=$ Etatdisziplin $=5$. 
Tabelle 21: Öffentliche Defizite/Überschüsse') in den Mitgliedstaaten in \% des Bruttoinlandsprodukts/ Bruttosozialprodukts

\begin{tabular}{|c|c|c|c|c|c|c|c|c|}
\hline Mitgliedstaat & 1980 & 1985 & 1986 & 1987 & 1988 & 1989 & 19902) & 19912) \\
\hline D & $-2,9$ & $-1,1$ & $-1,3$ & $-1,9$ & $-2,1$ & $+0,2$ & $-3,1$ & $-4,0$ \\
\hline B & $-9,2$ & $-8,6$ & $-8,9$ & $-7,1$ & $-6,4$ & $-6,3$ & $-5,9$ & $-5,9$ \\
\hline DK & $-3,3$ & $-2,0$ & $+3,4$ & $+2,5$ & $+0,3$ & $-0,6$ & $-1,3$ & $-1,6$ \\
\hline $\mathrm{F}$ & $-0,0$ & $-2,9$ & $-2,7$ & $-1,9$ & $-1,8$ & $-1,5$ & $-1,2$ & $-1,2$ \\
\hline GRL & $-2,9$ & $-13,5$ & $-10,7$ & $-12,3$ & $-15,3$ & $-18,4$ & $-18,3$ & $-16,7$ \\
\hline GB & $-3,4$ & $-2,7$ & $-2,2$ & $-1,3$ & $+1,0$ & $+0,9$ & $+0,1$ & $-0,2$ \\
\hline IRL & $-12,2$ & $-11,8$ & $-11,6$ & $-9,2$ & $-5,1$ & $-3,0$ & $-1,7$ & $-2,3$ \\
\hline I & $-8,5$ & $-12,5$ & $-11,7$ & $-11,1$ & $-10,9$ & $-10,2$ & $-10,0$ & $-9,5$ \\
\hline LUX & $-0,4$ & $+5,3$ & $+3,3$ & $+1,3$ & $+2,1$ & $+3,2$ & $+3,3$ & $+1,1$ \\
\hline NL & $-4,0$ & $-4,8$ & $-6,1$ & $-6,5$ & $-5,1$ & $-5,2$ & $-5,5$ & $-4,5$ \\
\hline $\mathbf{P}$ & - & $-10,1$ & $-7,2$ & $-6,8$ & $-5,4$ & $-3,8$ & $-6,0$ & $-5,6$ \\
\hline $\mathrm{E}$ & $-2,6$ & $-7,0$ & $-6,1$ & $-3,2$ & $-3,2$ & $-2,6$ & $-3,2$ & $-2,4$ \\
\hline
\end{tabular}

Quelle: Bundesministerium der Finanzen $=$ Finanzbericht $1991=307$. 
Tabelle 22: Staatsschulden ${ }^{1)}$ der Mitgliedstaaten der Europäischen Gemeinschaften in \% des Bruttoinlandsprodukts/Bruttosozialprodukts

\begin{tabular}{|lrrrrrrrr|}
\hline Mitgliedstaat & 1980 & 1985 & 1986 & 1987 & 1988 & $19892)$ & $1990^{3)}$ & $19913)$ \\
\hline D & 32,5 & 42,2 & 42,5 & 43,8 & 44,2 & 43,1 & 44,6 & 47,0 \\
B & 79,9 & 122,7 & 127,2 & 131,7 & 133,7 & 130,8 & 129,3 & 129,3 \\
DK & 33,5 & 65,7 & 59,3 & 56,9 & 55,7 & 54,9 & 55,3 & 55,8 \\
F & 37,3 & 45,5 & 45,7 & 47,5 & 47,4 & 46,9 & 46,7 & 46,5 \\
GRL & 27,7 & 57,9 & 58,6 & 64,6 & 72,1 & 79,6 & 83,4 & 86,5 \\
UK & 54,6 & 53,1 & 51,7 & 49,2 & 43,6 & 38,6 & 36,1 & 34,2 \\
IRL & 78,0 & 117,5 & 132,8 & 135,2 & 134,1 & 125,1 & 117,7 & 113,1 \\
I & 58,5 & 84,1 & 88,5 & 93,0 & 95,6 & 98,4 & 99,9 & 101,0 \\
LUX & 13,8 & 14,0 & 13,8 & 12,0 & 10,2 & 8,8 & 7,8 & 6,8 \\
NL & 45,9 & 69,6 & 71,3 & 75,2 & 78,1 & 80,9 & 83,0 & 84,6 \\
P & 37,1 & 69,5 & 68,4 & 72,7 & 78,7 & 71,5 & 67,8 & 64,7 \\
E & 18,5 & 47,3 & 48,1 & 48,5 & 43,9 & 43,1 & 43,2 & 43,0 \\
\hline 1) Bruttoschuld des Staatssektors. & & & & & & \\
2) Vorläufig. & & & & & & & \\
\hline
\end{tabular}

Quelle: Bundesministerium der Finanzen $=$ Finanzbericht $1991=307$. 
Zwar wird der finanzpolitische Spielraum mit der Beseitigung aller Kapitalverkehrsbeschränkungen und bei einem Verbot der Finanzierung der Haushaltsdefizite durch Geldschöpfung 96 infolge steigender Zinsbelastungen eingeschränkt, woraus sich ein gewisser Konsolidierungsdruck ergeben kann. Es bestehen jedoch Zweifel, ob dadurch die Koordinierung der Haushaltspolitik der Mitgliedstaaten immer rechtzeitig und problemlos zustande gebracht werden kann. Dies gilt vor allem dann, wenn die Staaten, die zu einer Konsolidierung gezwungen sind, damit rechnen dürfen, daß sie einen Teil der Last auf die gesamte Gemeinschaft übertragen können. Erwartungen in dieser Hinsicht werden durch Forderungen genährt, die Strukturfonds im Rahmen der Währungsunion kräftig aufzustocken. ${ }^{97}$ Auch wenn es in der Gemeinschaft keine Solidarhaftung für die Mitgliedstaaten gibt, kann der Druck auf die Gemeinschaft, die zur Konsolidierung gezwungenen Mitgliedstaaten mit (zusätzlichen) Hilfen zu stützen, beträchtlich werden. ${ }^{98}$

Es wäre daher sinnvoll, in den Verträgen bestimmte Regeln für die Haushaltspolitik der Mitgliedstaaten zu vereinbaren. Man könnte einen bestimmten Prozentsatz der Ausgaben für öffentliche Sachinvestitionen, der gesamtwirtschaftlichen Ersparnisbildung oder des Sozialprodukts, aber auch mehrere dieser Indikatoren (alternativ oder kumulativ) als Schranken für die Nettokreditaufnahme festlegen. "Wenn solche Grundsätze für die nationale Haushaltspolitik von den Mitgliedsländern der Gemeinschaft vertraglich vereinbart und von den Parlamenten ratifiziert werden, könnte dem Rat der Finanzminister auch die Aufgabe übertragen werden, die Regeln durch Beschlüsse zu konkretisieren und ... die Grenzen für diejenigen Länder zu erhö-

96 Über ein solches Verbot besteht weitgehend Einigkeit.

97 Vgl. Sachverständigenrat zur Begutachtung der gesamtwirtschaftlichen Entwicklung =Jahresgutachten $1989 / 90=414$ und Issing =Europäische Währungsintegration= 127.

98 Vgl. Sachverständigenrat zur Begutachtung der gesamtwirtschaftlichen Entwicklung $=$ Jahresgutachten 1989/90 $=414$ und Deutsche Bundesbank =Stellungnahme zur Errichtung einer $W W U=42$. Vor allem ist auch zu bedenken, daß ein einzelner "Sünder" vielleicht in die Knie gehen mag. "Schon drei oder vier würden indes eine trotzige Front bilden." Süddeutsche Zeitung vom 25.8.1990. 
hen, die bei Gefahr eines selbstverstärkenden Abschwungs keinen Spielraum mehr für die dann notwendige Aufstockung ihres Defizits haben." 99

Ausnahmeregelungen für den Rezessionsfall müssen jedoch so klar und begrenzt formuliert werden, daß sie die Defizitbeschränkung nicht aushöhlen. ${ }^{100}$ Die Begrenzung der Defizite durch Regelungen ist schließlich nur dann wirkungsvoll, wenn sie auch durchgesetzt werden kann. Ob dafür öffentlicher Druck der Gemeinschaft auf einen die Regel verletzenden Mitgliedstaat ausreicht, ist zweifelhaft. Wirksamer wären automatische Sanktionen, beispielsweise eine automatische Kürzung regionaler Transfers. ${ }^{101}$

Die bisherigen Überlegungen betrafen vor allem die letzte Stufe der Wirtschafts- und Währungsunion. Eine besondere Problematik weist jedoch die zweite Stufe des Entwicklungsprozesses auf, in der die Europäische Zentralbank neben den nationalen Zentralbanken besteht. ${ }^{102}$ Man muß sich darüber im klaren sein, daß eine teilweise Übertragung geldpolitischer Kompetenzen auf die Europäische Zentralbank nicht sinnvoll ist. Angesichts der divergierenden gemeinschaftlichen und nationalen Vorstellungen bleibt die Geldpolitik in

99 Sachverständigenrat zur Begutachtung der gesamtwirtschaftlichen Entwicklung =Jahresgutachten 1989/90= Ziff. 418. Für vertragliche Vorkehrungen einschließlich bindender Regelungen und Sanktionen für eine wirksame Haushaltsdisziplin in allen Mitgliedstaaten tritt auch die Deutsche Bundesbank ein. Deutsche Bundesbank =Errichtung einer $W W U=43$. Die Kommission hat ebenfalls schon Überlegungen angestellt, inwieweit die Erfahrungen in der Bundesrepublik Deutschland mit der Begrenzung des Haushaltsdefizits auf die Höhe der Investitionsausgaben für die Gemeinschaft zu verwenden sind. Dabei wurde insbesondere die Problematik einer klaren Definition der Vorgaben betont und darauf hingewiesen, daß die Abgrenzung der Investitionsausgaben als Bruttoinvestitionen zuzüglich investitionsbezogener Transfers an andere Haushalte, wie es der Praxis in der Bundesrepublik entspricht, nicht restriktiv genug wirkt, um einer "untragbaren Entwicklung der öffentlichen Schuld/BIP-Quote vorzubeugen". Kommission der EG = Länderstudien $1=56$.

100 Vgl. zu dieser Befürchtung Scheide, Trapp =Etatdisziplin $=5$.

101 So auch Neumann $=$ Gemeinsame Aufgaben $=94$.

102 Vgl. zu diesen Problemen auch Neumann $=$ Zweite Stufe $=336 \mathrm{ff}$. und Kloten $=$ Delors-Bericht $=258$. 
der Gemeinschaft unbestimmt und wohl kaum ausreichend auf Stabilität gerichtet. 103 Werden der Europäischen Zentralbank in der zweiten Phase keine geldpolitischen Kompetenzen übertragen, kommt sie in eine seltsame Lage:

"Das, was sie einüben müßte - nämlich die Anwendung des geld-
politischen Instrumentariums auf europäischer Ebene --, kann sie
nicht einüben, weil die geldpolitische Verantwortung in dieser
Phase bei den nationalen Notenbanken bleibt. Das, was sie in der
Ubergangsphase einüben könnte und nach dem Beschluß des Eu-
ropäischen Rates auch soll - eine Koordinierung der nationalen
Geldpolitiken -, braucht sie später nicht mehr, weil dann mit der
Ubertragung der geldpolitischen Verantwortung auf die europäi-
sche Zentralbank dieser Koordinierungsbedarf entfällt."

Durch das Nebeneinander europäischer und nationaler geldpolitischer Institutionen ist ein Gerangel um Kompetenzen unvermeidlich, durch das sich Reibungsverluste ergeben. Außerdem wird ein unangemessen hoher Personalbestand aufgebaut, weil die Europäische Zentralbank bereits in der Übergangsphase Mitarbeiter rekrutieren muß und es den nationalen Notenbanken aufgrund der ihnen verbliebenen geldpolitischen Verantwortung nicht möglich ist, ihren Mitarbeiterbestand zu verringern. ${ }^{105}$

All dies zeigt, daß eine unklare Kompetenzabgrenzung vermieden werden muß. Nur wenn klar ist, wie die Verantwortlichkeiten verteilt sind, können Aufgaben effizient erfüllt werden. Im nächsten Abschnitt wird deutlich, daß dies nicht nur für die Geldpolitik, sondern für viele weitere Aufgabenbereiche der Europäischen Gemeinschaften gilt.

$103 \mathrm{Vgl}$. Tietmeyer $=$ Voraussetzungen $=304$.

104 Vgl. Sachverständigenrat zur Begutachtung der gesamtwirtschaftlichen Entwicklung $=$ Jahresgutachten 1990/91 = Ziff. 486.

$105 \mathrm{Vgl}$. Sachverständigenrat zur Begutachtung der gesamtwirtschaftlichen Entwicklung =Jahresgutachten 1990/91 = Ziff. 486. 


\section{Das Problem der unklaren Aufgabenzuordnung}

In Teil $C$ dieser Arbeit wurde gezeigt, daß der Vertrag zur Gründung der Europäischen Gemeinschaft für Kohle und Stahl und der Vertrag zur Gründung der Europäischen Atomgemeinschaft als rechtsetzende Verträge eine präzise Abgrenzung der Aufgabenbereiche beinhalten. Der Vertrag zur Gründung der Europäischen Wirtschaftsgemeinschaft steckt dagegen als Rahmenvertrag nur in groben Umrissen diejenigen Bereiche $a b$, in denen sich eine Politik der Gemeinschaft entwickeln soll. Eine nähere Abgrenzung wird der Rechtsetzung der Gemeinschaftsorgane überlassen. ${ }^{1}$ Zwar können die Aufgaben in einem sich in der Entwicklung befindlichen föderativen System nicht immer detailliert und scharf abgegrenzt werden ${ }^{2}$, doch beläßt der Vertrag zur Gründung der Europäischen Wirtschaftsgemeinschaft durch die allzu weit und zum Teil sehr unpräzis gefaßten Begriffe in den Aufgabenkatalogen ${ }^{3}$ der Gemeinschaft einen großen Gestaltungsspielraum sowohl für den Bereich der aufgegriffenen Aufgaben als auch für die Intensität der Aufgabenerfüllung. ${ }^{4}$ Dieser Spielraum ist in jüngerer Zeit von der Kommission verstärkt dazu verwendet worden, Aufgaben an sich zu ziehen. Dadurch ist jedoch das Problem verschärft worden, daß dem föderativen System der Europäischen Gemeinschaften keine klare Aufgabenzuordnung zugrundeliegt.

Die Gemeinschaft nimmt nur sehr wenige Aufgaben "ausschließlich" wahr. Bei den meisten Aufgaben wird die Gemeinschaft ergänzend und unterstützend neben den Mitgliedstaaten tätig. Dabei wird der Finanzausgleich von der Kommission nicht als "Nullsummen-Kompetenzkonflikt" 5 zwischen den Europäischen Gemeinschaften und den Mitgliedstaaten interpretiert. Nach ihrer Vorstellung soll die Wahrnehmung von Aufgaben durch die Gemeinschaft nicht automatisch eine entsprechende Kompetenzverminderung auf nationaler

$1 \quad$ Vgl. Nittka $=$ Finanzierungssystem $=172$.

2 Vgl. dazu auch Arbeitsgruppe "Europäische Verfassung" =Institutionelle Weiterentwicklung $=15$.

3 So auch Stoiber $=$ Rechtsgemeinschaft $=545$.

$4 \quad$ Vgl. Andel $=$ Europäische Gemeinschaften $=313$.

$5 \quad$ Scharpf $=$ Politikverflechtungs-Falle $=325$. 
Ebene zur Folge haben. 6 Dies führt jedoch zu Problemen der Kompetenzverflechtung.

\section{Die fehlende Transparenz}

Die Verflechtung von Kompetenzen hat zur Folge, daß Entscheidungen und damit Verantwortlichkeiten nicht mehr klar zugeordnet werden können. Diese mangelnde Transparenz erschwert die öffentliche und die parlamentarische Kontrolle. Ohne eine entsprechende Kontrolle ist jedoch eine effiziente Erfüllung von Aufgaben gefährdet.

Die Verflechtung von Kompetenzen kann für die handelnden Politiker durchaus von Vorteil sein. Durch die Diffusion der Kompetenzen können sie sich nämlich der politischen Verantwortung für Fehlschläge und unpopuläre Entscheidungen entziehen. Gelungene Aktionen können dagegen von allen als Verdienst verbucht werden. "Dieser 'politisch-bürokratische Nutzen der Politikverflechtung' verzerrt die Präferenzen zum Nachteil von Vorhaben, die von einer Ebene alleine finanziert und verantwortet werden müßten. ${ }^{7}$ Insofern ist der Kompetenzverflechtung die Tendenz zur Ausweitung inhärent. ${ }^{8}$ Allerdings ist zu beachten, daß sich die Bürger, die die Zuständigkeiten nicht mehr durchschauen, zunächst immer an die nationalen Regierungen halten werden. Diese haften auch dann noch, wenn sie Zuständigkeiten verloren haben oder im Ministerrat überstimmt wurden. ${ }^{9}$

Werden Bund und Länder neben den Europäischen Gemeinschaften tätig, wie dies z. B. bei der Technologiepolitik der Fall ist, so sind die Programme und Maßnahmen der einzelnen Ebenen häufig nicht mehr überschaubar. Für Unternehmen und Forschungseinrichtungen steigen dadurch die Informationskosten

6 Vom Präsidenten der Kommission wird explizit hervorgehoben, daß die Frage nach der Trennlinie zwischen nationalen und Gemeinschaftskompetenzen falsch gestellt sei. Entsprechend seiner Idee der Komplementarität hat eben nicht jede Kompetenzausübung gleichzeitig einen Kompetenzverlust der nationalen Ebene zur Folge. Vgl. Delors $=$ Treffen mit den Ministerpräsidenten $=342$.

$7 \quad$ Scharpf $=$ Politikverflechtungs-Falle $=336$.

8 Da sich bei "konkurrierenden" Aufgaben ausgeprägte Bürokratien auf der Ebene der EG, des Bundes und z. T. auch der Länder finden, wird die Kompetenzverflechtung auch durch die fachlich spezialisierten "Ressortkumpaneien" gefördert. "Sie alle profitieren von den zusätzlichen Ressourcen und den zusätzlichen Zugangsmöglichkeiten zu politischen Entscheidungen ..." Scharpf = Politikverflechtungs-Falle= 349 .

Vgl. Kielmansegg $=$ Weg zur Politischen Union $=13$. 
erheblich. ${ }^{10}$ Überschneidungen bei den angebotenen Forschungsförderungsprogrammen führen zu Effizienzverlusten. Die mangelnde Überschaubarkeit erhöht zudem das Bedürfnis nach Vereinheitlichung oder Koordination, welche in der Regel durch die zentrale Instanz, also die Gemeinschaft, vorgenommen wird. Damit entsteht eine automatische Tendenz zur Zentralisierung. ${ }^{11}$

\section{Zielkonflikte - das Beispiel der Agrarpolitik}

Die Koordination der Maßnahmen unterschiedlicher Ebenen ist vor allem dann problematisch, wenn schon von vornherein unterschiedliche Zielsetzungen der nationalen und der gemeinschaftlichen Politik vorliegen. Dies soll am Beispiel der Agrarpolitik deutlich gemacht werden.

Die Agrarpolitik ist gekennzeichnet durch das Nebeneinander von europäschem Marktordnungssystem, das die Landwirtschaft vor allem gegen Konkurrenz aus Drittländern schützt, sowie nationalen Subventionen und Steuervergünstigungen, welche die nationale Landwirtschaft auch gegenüber Konkurrenten aus den Partnerländern wettbewerbsfähig zu machen versuchen. "Konflikte zwischen der Förderung der Erzeugung und dem Vorhandensein von strukturellen Uberschüssen stellen sich wegen der finanziellen Solidarität bei der Finanzierung der gemeinsamen Agrarpolitik auf nationaler und EG-Ebene unterschiedlich dar. Eine aus der Sicht der Gemeinschaft wegen des Anwachsens von strukturellen Überschüssen unerwünschte Maßnahme zur Verbesserung der landwirtschaftlichen Produktionsbedingungen kann aus nationaler Sicht noch vorteilhaft sein ...; denn die Vorteile der durchgeführten Agrarstrukturmaßnahmen, die sich beispielsweise niederschlagen konnen in Einkommenszuwächsen im landwirtschaftlichen Sektor, ... fallen dort an, wo diese Maßnahmen eingesetzt werden."

Es kann daher durchaus im Interesse eines Mitgliedstaates liegen, die nationale Produktion zu erhöhen, um auf diesem Wege stärkeren Nutzen aus der gemeinsamen Finanzierung der Agrarpolitik zu ziehen. ${ }^{13}$ Mißt man den Vorteil nationaler Strategien vor allem an der Veränderung der Finanztransfers, dann zieht jener Mitgliedstaat den größten Vorteil aus der Externalisierung von Folgekosten der nationalen Agrarpolitik, der seine Agrarproduktion am stärksten

10 Das "Förderdickicht" wird nur noch von Experten durchschaut. So hat ein deutscher Steuerberater einen 3000seitigen Info-Band herausgegeben, der die Fördermöglichkeiten der Gemeinschaft, aber auch des Bundes und der Länder auflistet. Vgl. Kessler $=$ Wildes Dickicht $=50$.

11 Vgl. Starbatty, Vetterlein $=$ Technologiepolitische Aufgabenteilung $=102$.

12 Thoroe =Europäische Agrarpolitik $=115 \mathrm{f}$.

13 Vgl. dazu auch Petersen $=$ Agrarpolitik $=44$. 
ausweitet und damit am meisten zur Verschärfung der Überschußprobleme auf Gemeinschaftsebene beiträgt. ${ }^{14}$

Auch die neueren Regelungen in der Agrarpolitik haben diese Problematik nicht entschärft, sondern nur auf eine andere Ebene verlagert. Am Beispiel des 1988 beschlossenen Programms zur Flächenstillegung wird dies besonders deutlich: Um eine Reduktion der landwirtschaftlichen Produktion zu erreichen, werden die Mitgliedstaaten verpflichtet, Prämien für die Stillegung von Anbauflächen anzubieten, die sie innerhalb eines bestimmten Rahmens selbst festsetzen können. ${ }^{15}$ Die nationalen Haushalte werden durch diese Regelung einerseits belastet, andererseits durch die sich auf der Gemeinschaftsebene verringernden Überschußkosten entsprechend dem nationalen Anteil am Gemeinschaftshaushalt entlastet.

Die Maßnahme ist aus nationaler Sicht dann als besonders günstig zu beurteilen, wenn Partnerländer genügend Anreize zur Flächenstillegung geben, der Mitgliedstaat selbst dagegen nur geringe Anreize bietet. Der einzelne Mitgliedstaat ist gut beraten, durch Flächenstillegungen möglichst wenig Produktion zu reduzieren. "Im Extremfall könnten Brachflächen offiziell stillgelegt werden. In diesem Fall erhält dieser Mitgliedstaat einen Sozialprodukttransfer von den Mitgliedsländern. Dagegen wird der Verlust an Sozialprodukt besonders hoch sein, wenn durch eine hohe Prämie und verbunden damit einem hohen nationalen Finanzierungsanteil an der Prämie ertragreiche Böden aus der Produktion genommen werden. Demnach bestehen starke Anreize, sich auf nationaler Ebene nicht entsprechend der EG-Zielsetzung zu verhalten." 16

Das Gemeinschaftsziel wird außerdem nicht gerade dadurch gefördert, daß der von der Gemeinschaft gezahlte Anteil an den Beihilfen mit zunehmender Höhe des vom Mitgliedstaat festgelegten Förderungssatzes abnimmt, also "regressiver Art" ist. ${ }^{17}$ Für die Mitgliedstaaten besteht daher kein Anreiz, die Sätze hoch festzusetzen.

14 Vgl. Thoroe =Europäische Agrarpolitik $=116$.

15 Eine vergleichende Darstellung der Beihilfen in Deutschland, Italien und Großbritannien findet sich in Europäischer Rechnungshof $=$ Jahresbericht $1989=92$.

16 Koester, Terwitte $=$ Politikversagen $=134$.

17 So der Europäische Rechnungshof $=$ Jahresbericht $1989=97$. 


\section{Koordinierungsprobleme - das Beispiel der Regionalpolitik}

Sind mehrere Ebenen für eine Aufgabe zuständig, so müssen die jeweiligen Aktivitäten laufend koordiniert werden. Welche Schwierigkeiten dabei auftreten, wird am Beispiel der Regionalpolitik besonders deutlich.

Die Förderung durch den Europäischen Fonds für regionale Entwicklung war schon immer an das "untere" mitgliedstaatliche Instrumentarium gebunden, in der Bundesrepublik Deutschland an die Rahmenplanung der Gemeinschaftsaufgabe "Verbesserung der regionalen Wirtschaftsstruktur". 18 Wie oben beschrieben, stellen nationale Behörden zunächst regionale Pläne auf, die auf der Ebene zu erarbeiten sind, die dazu für am geeignetsten gehalten wird; in der Bundesrepublik Deutschland sind dies die Länder. Auf der Grundlage dieser Pläne erarbeitet die Kommission in Verhandlungen mit dem jeweiligen Mitgliedstaat die "Gemeinschaftlichen Förderkonzepte". Diese stellen die Antwort der Kommission auf den in den Plänen dargestellten Handlungsbedarf dar.

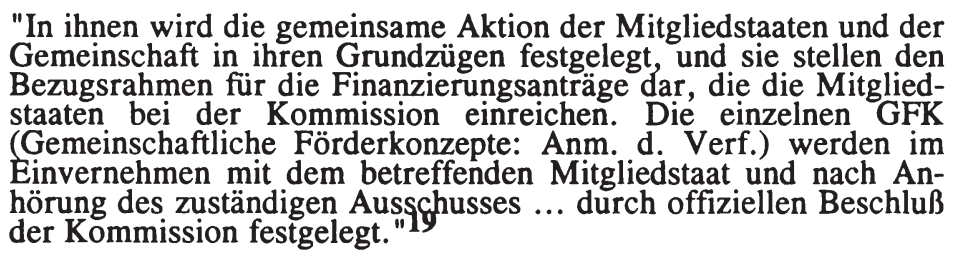

Danach erst können die Mitgliedstaaten konkrete Mittel beantragen. Das Verfahren soll eine enge Konzertierung zwischen der Kommission, dem betreffenden Mitgliedstaat und den von ihm bezeichneten, auf nationaler, lokaler oder sonstiger Ebene zuständigen Behörden sicherstellen. Dabei wird die Partnerschaft zwischen den beteiligten Ebenen betont. ${ }^{20}$ Das Verfahren ist jedoch nicht unproblematisch. Es erfordert "bei Bund und Ländern zusätzliche Koordinierung bei Vorbereitung, Durchführung und Kontrolle gemeinschaftlich finanzierter Aktionen, da sich die Gemeinschaft mit mehreren Fonds und Finanzierungsinstrumenten beteiligen kann. Dies bedeutet einen erheblichen admini-

18 Vgl. vor allem Rat der EG =EFRE-Verordnung 1984=1ff. Nach Art. 11 Abs. 3 und Art. 17 Abs. 3 dieser Verordnung erfolgt die Beteiligung des EFRE vorwiegend zugunsten der Gebiete, die aufgrund der nationalen Rahmenplanung als Fördergebiete ausgewiesen sind.

19 Kommission der EG =Reform der Strukturfonds 1989= 30 .

20 Vgl. Kommission der EG = Reform der Strukturfonds $1989=15$. 
strativen Aufwand ..."21 Da sich in den letzten Jahren immer mehr abgezeichnet hat, daß die Mittelzuflüsse aus den Strukturfonds für die Bundesrepublik Deutschland relativ gering ausfallen ${ }^{22}$, stellt sich hier die Frage nach dem angemessenen Verhältnis zwischen Planungs- und Verwaltungsaufwand und den zu erwartenden Mittelrückflüssen.

Einen hohen bürokratischen Aufwand gibt es jedoch nicht nur bei den Mitgliedstaaten, sondern auch für die Kommission, denn sie muß die eingereichten Pläne im Detail prüfen, wobei die große Ferne zu den Problemen nicht gerade hilfreich ist. ${ }^{23}$ Außerdem müssen die von den Europäischen Gemeinschaften mitfinanzierten Projekte kontrolliert werden. ${ }^{24}$ Auch der zeitliche Bedarf des Verfahrens gibt Anlaß zur Kritik. Die Anfertigung der Pläne durch die Länder, das Aufstellen der gemeinschaftlichen Förderkonzepte und das anschließende Einreichen konkreter Anträge für die Mittelverwendung läßt nicht auf eine schnelle und damit flexible Strukturpolitik hoffen. ${ }^{25}$

Insgesamt sollte bei der Kompetenzverteilung zwischen der Gemeinschaft und den nationalen Ebenen stärker darauf geachtet werden, daß klargestellt ist, wie die Verantwortung verteilt ist. Dazu müßten von vornherein die Aufgaben der Gemeinschaft präziser umrissen werden, als dies derzeit in den Verträgen geschieht. Parallele Aktivitäten, wie etwa bei der Forschungsförderung, sollten weitgehend vermieden werden. Nimmt die Gemeinschaft im selben Politikbereich ergänzend zu den nationalen Ebenen Aufgaben wahr, muß sie die nationalen Maßnahmen und die ihnen zugrundeliegenden Zielsetzungen in ihr Kalkül miteinbeziehen. Zum einen bedeutet dies, daß die Gemeinschaftsmaßnahmen nicht so gestaltet sein dürfen, daß nationale Ebenen einen Anreiz erhalten, sich genau entgegen der gemeinschaftlichen Zielsetzung zu verhalten. Zum anderen müssen die nationalen und gemeinschaftlichen Aktivitäten koordiniert werden, ohne daß zu hohe administrative Kosten anfallen oder durch eine zu lange Dauer des Koordinationsprozesses eine flexible Politik unmöglich wird.

21 Planungsausschuß für regionale Wirtschaftsstruktur $=18$. Rahmenplan $=13$.

22 Dies wird sich in den nächsten Jahren dadurch etwas ändern, daß in die neuen Länder zusätzliche Mittel fließen. Bisher sind für den Zeitraum 1991-1993 Strukturfondsmittel in Höhe von insgesamt 3 Mrd. ECU für die neuen Länder vorgesehen. Vgl. Presse- und Informationsamt der Bundesregierung $=$ Einbeziehung $=13$.

23 Vgl. dazu die Ausführungen unter D. I. 3. b).

24 Vgl. Karl-Bräuer-Institut des Bundes der Steuerzahler =EG-Finanzen= 72.

25 Zur zeitlichen Dauer der einzelnen Verfahrensschritte vgl. Kommission der EG =Reform der Strukturfonds $1989=39$. 


\section{Probleme der Ausgabenverteilung}

\section{Die Einteilung in obligatorische und nicht-obligatorische Ausgaben}

Die Ausgaben der Gemeinschaft sind durch die ungewöhnliche Einteilung in obligatorische und nicht-obligatorische Ausgaben gekennzeichnet. Diese Unterscheidung ist von vornherein deshalb problematisch, weil sie häufig nicht eindeutig vorzunehmen ist. Die Begriffe sind nicht im Vertragstext definiert, so daß sich Rat und Europäisches Parlament bisher jeweils ad-hoc auf eine entsprechende Klassifizierung der Mittel einigen mußten. ${ }^{1}$ Da die Aufteilung eng mit der Teilung der Haushaltsbefugnis zwischen Rat und Parlament zusammenhängt, kam es bei der Frage der Abgrenzung immer wieder zu Konflikten zwischen beiden Zweigen der Haushaltsbehörde. ${ }^{2}$ Zur Zeit werden als obligatorische Ausgaben eingestuft: ${ }^{3}$

- die Ausgaben im Rahmen der Agrarmarktordnung,

- die Erstattungen und Beihilfen an die Mitgliedstaaten,

- verschiedene, hauptsächlich der Fischereipolitik und der Zusammenarbeit mit Drittländern zurechenbare Haushaltslinien, die den finanziellen Verpflichtungen aus den Abkommen der Gemeinschaft mit Drittländern entsprechen.

Zu den nicht-obligatorischen Ausgaben zählen:

- die Ausgaben im Rahmen der Strukturfonds,

- die Interventionsmittel in den Bereichen Energie, Industrie und Forschung,

- fast sämtliche Verwaltungausgaben.

Diese Einteilung ist nicht sehr logisch; denn "auch Ausgaben, deren Grund und Höhe erst durch den Haushaltsplan determiniert werden (Summe der Beamtengehälter, Mieten) und die als nicht-obligatorisch eingestuft werden, müssen aufgrund bestehender Verpflichtungen der Gemeinschaftsorgane geleistet

1 Vgl. Beutler u. a. =Europäische Gemeinschaft $=167$.

2 Vgl. Kommission der EG $=$ Öffentliche Finanzen $=67$.

3 Vgl. Kommission der EG $=$ Öffentliche Finanzen $=68$. 


\section{Probleme der Ausgabenverteilung}

werden. Umgekehrt liegen die - als obligatorisch eingestuften - Erstattungen für die Landwirtschaft der Höhe nach nicht fest." 4

Das eigentliche Problem der Aufteilung ist jedoch nicht die Abgrenzung, sondern die Tatsache, daß sie den Kern für schwerwiegende finanzielle Fehlentwicklungen in sich birgt. Da das Europäische Parlament nur Haushaltsbefugnisse im Bereich der nicht-obligatorischen Ausgaben hat, hat es verständlicherweise die Neigung, diesen Bereich besonders auszuweiten. Demgegenüber wird der Ministerrat dazu neigen, wegen seiner Kompetenzen für die obligatorischen - d. h. insbesondere für die Agrarausgaben - diese Haushaltstitel entsprechend stark zu dotieren und die übrigen Bereiche zu vernachlässigen. ${ }^{5}$ Für beide Organe ist die Höhe der von ihnen gestaltbaren Ausgaben damit vor allem eine Frage der Gewichtung von Kompetenzen. Da keine direkte Verantwortung für die Finanzierung dieser Ausgaben gegenüber dem Bürger (oder konkreter dem Steuerzahler) besteht ${ }^{6}$, liegt es im Interesse des jeweiligen Organs, die eigenen Ausgaben zu erhöhen. Eine sparsame Haushaltsführung ist unter diesen Umständen unmöglich; die Teilung der Ausgaben in obligatorische und nicht-obligatorische, und die ihr zugrundeliegende Aufteilung der Haushaltsbefugnisse, fördert die Tendenz zur Aufblähung des Gemeinschaftshaushaltes. Es wäre daher sinnvoll, diese Trennung aufzugeben und ein einheitliches Haushaltsverfahren festzulegen, das ausschließlich dem Parlament die Entscheidung über den Haushalt überträgt.

\section{Die Dominanz der Agrarausgaben}

\section{a) Die grundsätzliche Problematik}

Im Rahmen der grundsätzlichen Überlegungen zur sinnvollen Gestaltung des Finanzausgleichs wurde gezeigt, daß ein Zusammenhang zwischen den der jeweiligen politischen Entscheidungsebene zugestandenen Aufgaben und ihren Ausgaben bestehen sollte. ${ }^{7}$ Berücksichtigt man, daß die Agrarmarktpolitik neben der tarifären Außenhandelspolitik der einzige Bereich ist, in dem die Europäischen Gemeinschaften sogenannte ausschließliche Kompetenzen besitzen -

\footnotetext{
4 Beutler u. a. =Europäische Gemeinschaft $=164$.

$5 \quad \mathrm{Vgl}$. Biehl, Winter $=$ EG-Finanzierung $=73$.

6 Vgl. dazu genauer D. IV. 2. c).

7 Vgl. B. II. 3.
} 
also nicht nur nationale Maßnahmen ergänzen - so ist ein starkes finanzielles Engagement der Gemeinschaft in diesem Bereich durchaus verständlich. ${ }^{\mathbf{8}}$

Das Volumen der Finanzierung der Agrarpolitik ist jedoch nicht nur abhängig von den Kompetenzen, die der Gemeinschaft übertragen worden sind, sondern auch von der Ausgabenintensität der zum Einsatz gelangten Instrumente. ${ }^{9}$ Dies wird daran deutlich, daß es Aufgaben gibt, die den Europäischen Gemeinschaften trotz weitreichender Kompetenzen relativ geringe Ausgaben verursachen (z. B. die Wettbewerbspolitik oder die tarifäre Außenhandelspolitik). Es ist somit falsch, den hohen Anteil der Agrarausgaben an den Gesamtausgaben ("Agrarlastigkeit der Ausgaben") damit zu rechtfertigen, daß die Europäischen Gemeinschaften hier ausschließliche Kompetenzen besitzen.

Das Volumen des Haushalts wurde bisher weitgehend durch das Ausmaß der Überproduktion im Agrarsektor bestimmt, und für die Verteilung der Ausgaben war in erster Linie der örtliche Anfall dieser Überproduktion ausschlaggebend. Diese problematische Vorgehensweise hat zur Folge, daß die hohen Agrarmarktausgaben zu einem Hemmschuh für die Entwicklung bedeutsamer Gemeinschaftsausgaben in anderen Bereichen geworden sind. ${ }^{\mathbf{1 0}}$ Das Konnexitätsprinzip kann jedoch nicht voll verwirklicht werden, wenn für eine Aufgabe fast alle Ausgaben verwendet werden, so daß für andere Aufgaben nicht mehr genügend Mittel bereitstehen.

Die Agrarausgaben konnten nur deshalb so stark ansteigen, weil sie sich einer Budgetkontrolle entziehen. Für die Agrarpreisbeschlüsse ist der Agrarministerrat zuständig. Bei seinen Entscheidungen sucht dieser verständlicherweise einen Ausgleich zwischen verschiedenen nationalen agrarpolitischen Positionen. Zuwenig berücksichtigt werden dagegen die Interessen der Verbraucher und der Steuerzahler, die bei diesen Entscheidungen nicht vertreten sind. ${ }^{11}$

Da sich der Agrarministerrat keine Gedanken über die Finanzierung zu machen braucht, gibt es für ihn im Grunde keine Grenzen für die entstehenden Ausgaben. Die Tatsache, daß der Agrarministerrat die Finanzierungsseite nicht

$8 \mathrm{Vgl}$. Mohr $=$ Finanzprobleme $=10$.

9 Vgl. Petersen $=$ Agrarpolitik $=38$ und Reding $=$ Problematik eines Finanzausgleichs $=$ 211.

10 Vgl. Nittka $=$ Finanzierungssystem $=255$ f. und Spahn $=$ Zukunft der Finanzen $=3$.

11 Vgl. Biehl, Winter $=$ EG-Finanzierung $=74$. 
beachten muß, unterscheidet sich noch nicht grundsätzlich von nationalen $\mathrm{Ge}$ pflogenheiten. Auch die einzelnen nationalen Ressortminister brauchen sich um die Finanzierung ihrer konkreten Ausgaben nicht zu kümmern; vielmehr werden im Sinne des Non-Affektationsprinzips alle Einnahmen zur Deckung aller Ausgaben verwendet, so daß auch hier keine Zurechnung bestimmter Einnahmen zu bestimmten Ausgaben erfolgt. Durch diesen Grundsatz soll sichergestellt werden, daß eine gewisse Flexibilität bei der Finanzierung sich verschiebender Aufgabenbereiche gewährleistet wird. Bei den nationalen Haushalten liegt allerdings ein überwiegend aus Steuermitteln finanziertes und in allen Teilen parlamentarisch kontrollierbares Budget vor. Diese Voraussetzungen für die Anwendung des Non-Affektationsprinzips sind beim Haushalt der Europäischen Gemeinschaften nicht gegeben. ${ }^{12}$ Während in den Mitgliedstaaten den Ausgabenwünschen der verschiedenen Ressorts durch den jeweiligen Finanzminister Grenzen gesetzt werden, wobei der Finanzminister eine gewisse Vormachtstellung ${ }^{13}$ einnimmt, hat der Finanzministerrat der Europäischen Gemeinschaften keine entsprechende Funktion. So können sich die Agrarminister ihre Ausgaben sozusagen selbst genehmigen. ${ }^{14}$

Die im Haushalt angesetzten Beträge für die Agrarausgaben haben eher den Charakter von Vorschlägen als von wirksamen Limitierungen. Daß dies so ist, liegt nicht nur an den Entscheidungen des Agrarministerrates, sondern auch daran, daß die tatsächlichen Ausgaben von einer Reihe exogener, also nicht von den Organen der Gemeinschaft beeinflußbaren Faktoren abhängen. Selbst wenn der Agrarministerrat bei der Festsetzung der Interventionspreise den Budgetrahmen beachtet, ist nicht auszuschließen, daß über nicht kalkulierbare Angebotsreaktionen die Mengen ausgeweitet werden oder durch Veränderungen der Weltmarktpreise oder des Dollarkurses zusätzliche Ausgaben erfor-

12 Vgl. Spahn $=$ Zukunft der Finanzen $=4$.

13 Diese Vormachtstellung ist teilweise gesetzlich verankert (etwa in der Bundesrepublik Deutschland), teilweise beruht sie jedoch auch auf Tradition und/oder dem persönlichen Durchsetzungsvermögen. Vgl. Senf $=$ Kurzfristige Haushaltsplanung $=382 \mathrm{f}$.

14 Ähnliche Probleme ergeben sich im übrigen auch bei anderen Politikbereichen, wenn Fachminister ohne die Finanzminister ausgabenintensive Beschlüsse fassen. So hat das Europäische Parlament gerügt, daß die EG-Außenminister finanzielle Zusagen an Drittländer gegeben haben, ohne sich über die Finanzierung Gedanken zu machen. Auch wenn Hilfsmaßnahmen politisch notwendig sind, muß klar sein, daß diese mit einer Erhöhung der Einnahmen verbunden sind. Das Parlament ist nicht bereit, laufende Programme zu kürzen und fordert, daß der Ministerrat für entsprechende Ausgaben eine Änderung der verschiedenen Finanzinstrumente, vor allem einen Nachtragshaushalt, vorlegen muß. Vgl. dazu Langes $=E G$ kein Privatunternehmen $=16$. 
derlich werden. ${ }^{15}$ Es gibt zwar auch in den nationalen Haushalten Ausgabenkategorien, die als rechtlich verbindlich anzusehen sind und daher zumindest tendenziell den finanziell gegebenen Rahmen übersteigen können. Doch machen solche Ausgaben in nationalen Budgets nicht annähernd zwei Drittel der Ausgaben aus. ${ }^{16} \mathrm{Da}$ entsprechend dem Non-Affektationsprinzip alle Ausgaben der Gemeinschaften durch alle Einnahmen gedeckt werden sollen, führen Steigerungen der Agrarmarktausgaben durch Faktoren, die die Haushaltsorgane nicht beeinflussen können, dazu, daß die gegebenen Mittel für andere Ausgaben nicht in ausreichendem Maße zur Verfügung stehen.

\section{b) Ansätze zur Reform der Agrarmarktpolitik}

Die Asymmetrie der Ausgaben der Europäischen Gemeinschaften wurde immer dann als Problem erkannt, wenn die Grenzen der Finanzierung des Gemeinschaftshaushaltes erreicht wurden. Erste Korrekturen an der Agrarmarktordnung wurden im Rahmen des Zucker- und des Milchmarktes vorgenommen. Dabei stand das Instrument der Erzeugerabgaben im Mittelpunkt der Reformansätze.

Auf dem Milchmarkt wurde schon mit den Preisabschlüssen für das Wirtschaftsjahr 1977/78 eine Erzeugerabgabe eingeführt. ${ }^{17}$ Auf die an die Molkereien gelieferte Milch wurde ein Satz von 1,5\%18 des Milchpreises erhoben, der von den Molkereien abzuführen war. Landwirte in benachteiligten Regionen waren davon ausgenommen. Diese Abgabe konnte ein weiteres Anwachsen der Überschüsse nicht verhindern. Als es im Jahre 1984 zu einer erneuten Haushaltskrise kam, bei der wie so oft grundsätzliche Reformen ausblieben, wurde ein System der Produktionsquoten für Milch beschlossen. ${ }^{19}$ Dabei wurden nationale Quoten für die einzelnen Mitgliedstaaten ausgehandelt. Die weitere Ausgestaltung des Quotensystems blieb weitgehend den Mitgliedstaaten überlassen.

15 Vgl. Spahn $=$ Zukunft der Finanzen $=3$.

16 Vgl. Spahn $=$ Zukunft der Finanzen $=2$.

17 Vgl. Rat der EG = Mitverantwortungsabgabe für Milch 1977=6f.

18 Der Satz wurde im Laufe der Zeit mehrfach geändert. Vgl. Thoroe =EG-Produktionssteuern $=354$. Zur Zeit beträgt er allerdings wieder $1,5 \%$ des Milchrichtpreises. Vgl. Kommission der EG =Agrarpreise 1991/92= 55 .

19 Vgl. Rat der EG = Marktorganisation für Milch 1984= 10ff. 


\section{Probleme der Ausgabenverteilung}

In der Bundesrepublik Deutschland entschied man sich für einzelbetriebliche Quoten $^{20}$, bei denen jedem Milcherzeuger eine individuelle Referenzmenge zugewiesen wird. Für Mengen, die diese Quoten überschreiten, muß eine Abgabe in Höhe von $75 \%$ des Milchrichtpreises gezahlt werden. Es gibt also zur Zeit zwei Erzeugerabgaben auf Milch: eine Grundabgabe auf die gesamte Produktion und eine Zusatzabgabe auf die Grenzproduktion, d. h. auf die Produktion, die die Referenzmenge überschreitet. ${ }^{21}$ Die Milchquote war kein Erfolg: Die Garantiemengen konnten nicht eingehalten werden und der Selbstversorgungsgrad bei Milcherzeugnissen liegt noch immer bei ca. $113 \% .22$

Auf dem Zuckermarkt gilt seit 1981 folgende Regelung: Es werden Produktionsquoten festgesetzt; A-Quoten mit uneingeschränkter Preisgarantie und BQuoten mit eingeschränkter Preisgarantie. Für Mengen, die diese Quoten überschreiten, gibt es keine Preisgarantien. Die A-Quoten und B-Quoten sind höher festgesetzt als der Zuckerverbrauch in der Gemeinschaft. Um die Verwertung entsprechender Überschüsse finanzieren zu können, werden Erzeugerabgaben erhoben. Dabei wird auf die Gesamtquote (A-Quote und B-Quote) eine Grundabgabe in Höhe von $2 \%$ des garantierten Interventionspreises auferlegt. Die verbleibenden Kosten werden allein auf die B-Quote umgelegt, wobei die Höchstabgabe auf $30 \%$, in Ausnahmefällen auf $37,5 \%$ begrenzt ist. Daneben wird eine Lagerkostenabgabe erhoben, die die durchschnittlichen Kosten der Lagerhaltung abdecken soll und die von den Zuckerfabriken beim Verkauf von Zucker erhoben wird. ${ }^{23}$

Daß hinter den auf dem Zuckermarkt und auf dem Milchmarkt eingeführten Erzeugerabgaben keine schlüssige Konzeption steht, sondern nur Haushaltskrisen kurzfristig überwunden werden sollten, läßt sich schon daran erkennen, daß die beiden Abgaben, obwohl sie sehr ähnlich konzipiert sind, im Haushalt unterschiedlich erfaßt werden. Während die Zuckerabgaben zu den eigenen Einnahmen zählen, werden die Erträge der Milchabgaben mit den Interventionsausgaben des Milchmarktes verrechnet, d. h. es werden Nettoausgaben

20 Die andere Möglichkeit sind Molkereiquoten. "Entschließt sich ein Land für KäuferQuoten (Molkerei-Quoten), dann muß für Überschußmengen eine Zusatzabgabe in Höhe von $100 \%$ des Milchrichtpreises gezahlt werden, wobei der abgabenpflichtige Käufer die Belastung über den Erzeugerpreis auf diejenigen Erzeuger abzuwälzen hat, die ihre Lieferungen erhöht haben." Thoroe $=$ EG-Produktionssteuern $=354$.

21 Vgl. Thoroe $=$ EG-Produktionssteuern $=355$.

22 Vgl. = Agrarbericht $1991=83 \mathrm{f}$. und Schrader $=$ Agrar - und Haushaltspolitik $=7$.

23 Vgl. Rat der EG = Marktorganisation für Zucker 1981= 4ff. 
verbucht, was gegen den Grundsatz der Vollständigkeit des Budgets verstößt. ${ }^{24}$

Im Mai 1986 führte der Ministerrat eine Mitverantwortungsabgabe für Getreide ein. ${ }^{25}$ Der Abgabesatz wurde auf 3\% des Interventionspreises für zur Brotherstellung geeigneten Weichweizen festgelegt und von dem den Erzeugern gezahlten Preis abgezogen. Durch eine Änderung ${ }^{26}$ wurde bestimmt, daß ab 1988 die Abgabepflicht dem Erstkäufer von Getreide aufzuerlegen ist. Die Grundabgabe wurde durch eine Zusatzabgabe in Höhe von bis zu 3\% des Interventionspreises ergänzt. Den Kleinerzeugern wurden schon ab 1986 direkte Beihilfen als Ausgleich für die Mitverantwortungsabgabe gewährt. ${ }^{27}$

Eine Untersuchung des Europäischen Rechnungshofes über die Mitverantwortungsabgabe kommt zu dem Schluß, daß sie nicht zu einer Verbesserung des Marktgleichgewichts beigetragen hat. Die Produktion stieg nach der Einführung tendenziell weiter an, gelegentlich rückläufige Produktionszahlen fielen sogar schwächer aus als jene, die auch vor der Einführung der Mitverantwortungsabgabe gelegentlich eintraten. ${ }^{28}$ Daneben verweist der Rechnungshof auf die großen Umsetzungsschwierigkeiten, die die Maßnahme nicht wirksam werden ließen. ${ }^{29}$

Der große Nachteil der Erzeugerabgaben lag darin, daß sie zunächst den Druck der leeren Kassen auf die Agrarpolitik milderten und so eine grundlegende Agrarreform hinausschoben. Anstelle einer solchen grundsätzlichen Reform wurde das Interventionssystem immer weiter ausgebaut: Auf die Regulierung der Preise folgt die Regulierung der Mengen. Hier zeigt das Interventionssystem seine unerbittliche Logik: Um eine Form des Interventionismus aufrecht $\mathrm{zu}$ erhalten, müssen neue Interventionen folgen. ${ }^{30}$

Nach einer weiteren Haushaltskrise 1988 wurde erneut über eine Agrarreform beraten. Der Europäische Rat legte im Februar 1988 fest, daß die jährliche

24 Vgl. dazu auch Thoroe =EG-Produktionssteuern $=356$.

25 Vgl. Rat der EG = Marktorganisation für Getreide 1986=29ff.

26 Vgl. Rat der EG = Marktorganisation für Getreide $1988=7 \mathrm{ff}$.

27 Vgl. Rat der EG =Kleinerzeuger im Getreidesektor 1986= $1 \mathrm{ff}$.

28 Vgl. Europäischer Rechnungshof $=$ Jahresbericht $1989=86$.

29 Vgl. Europäischer Rechnungshof $=$ Jahresbericht $1989=88$.

30 Vgl. dazu auch Thoroe $=$ EG-Produktionssteuern $=365$. 
Steigerungsrate der Ausgaben der Abteilung Garantie des Agrarfonds 74\% der jährlichen Steigerungsrate des Bruttosozialproduktes der Gemeinschaft nicht überschreiten darf ("Agrarleitlinie"). ${ }^{31}$ Dagegen sollten die Strukturfonds stark aufgestockt werden. Insgesamt sollte der Anteil der Ausgaben der Abteilung Garantie des Agrarfonds bis 1992 auf 56,1\% zurückgeführt werden. 32

Um dies zu erreichen, wurden über die bis dahin bestehenden Erzeugerabgaben hinaus sogenannte Stabilisierungsmechanismen in die Agrarvorschriften eingebaut. Sie betrafen 1989 ca. die Hälfte der Agrarerzeugung. ${ }^{33}$ Obwohl die einzelnen Stabilisierungsmechanismen auf die Besonderheiten der jeweiligen Erzeugung abgestimmt sind, läßt sich doch ein gleiches Grundkonzept feststellen: "Übersteigt die Erzeugung eine vorher festgesetzte Höhe, so wird das entsprechende Stützungsniveau automatisch gesenkt." ${ }^{34}$ Diese Senkungen sind nicht an Ministerratsbeschlüsse gebunden, sondern es obliegt der Kommission, nach Prüfung aller vorliegenden Informationen eine Überschreitung der Garantiehöchstmenge festzustellen und die Senkung des Stützniveaus nach genau festgelegten Regeln zu beschließen. In diesem Sinne arbeitet der Mechanismus weitgehend automatisch. ${ }^{35}$ Im Grunde handelt es sich bei den Stabilisatoren um eine zweifache Preisdifferenzierung: Der Preis wird nur für den Produzenten gesenkt, nicht aber für den Verbraucher. Die Regelung wirkt damit nicht anders als eine spezielle Verbrauchsteuer. ${ }^{36}$

Die Stabilisatoren stellen nur einen ersten Schritt zu mehr Markt im Agrarbereich dar, denn jede Mengenvorgabe ist willkürlich und die Steuerungsfunktion der Preise bleibt weiterhin außer Kraft gesetzt. Durch die Stabilisatoren wird nur das Niveau der Stützung, nicht aber das Prinzip der Agrarmarktregulierung geändert. Produktionsanreize bestehen fort. Die Nachfragestruktur wird auch weiterhin nicht berücksichtigt. Dagegen führen die Stabilisatoren zu einem erheblichen bürokratischen Aufwand.

31 Vgl. Europäischer Rat $=$ Schlußfolgerungen $1988=225$.

32 Vgl. Stahl $=$ Mittelfristige Finanzplanung $=422$.

33 Eine Übersicht über die derzeitigen Stabilisatoren gibt Kommission der EG =Agrarpreise 1991/92 = 19ff.

34 Kommission der EG = Lage der Landwirtschaft 1988=15.

35 Vgl. Kommission der EG =Lage der Landwirtschaft $1988=15$.

36 Vgl. Koester, Terwitte $=$ Politikversagen $=132$. 
Als flankierende Maßnahmen zur Begrenzung der landwirtschaftlichen Produktion und zur sozialen Absicherung des Strukturwandels wurden Programme zur Stillegung von Anbauflächen und Extensivierung, Vorruhestandsregelungen für ältere Landwirte sowie Einkommensbeihilfen vorgesehen. ${ }^{37}$ Die Stillegung von Anbauflächen läßt keine sehr große Entlastung des Haushaltes erwarten. "Zum einen dürfte der tatsächlich stillgelegte Flächenumfang geringer sein als der angegebene. Zum anderen werden ... vorrangig Grenzflächen aus der Produktion genommen und auf den verbleibenden Flächen ist eine Intensivierung zu erwarten. "38 Dies bestätigt der Europäische Rechnungshof: "In einigen einzelstaatlichen Leitlinien wird den Landwirten sogar empfohlen, die Stillegung von Flächen mit schlechter Bodenqualität, Krankheits-, Schädlingsoder Unkrautproblemen und von Flächen, deren Standort Anbauprobleme mit sich bringt, in Betracht zu ziehen. "39 In der Bundesrepublik Deutschland wurden in einigen Ländern Streifen von fünf und zehn Metern Breite als förderungswürdig genehmigt. "Diese Streifen sind ausnahmslos die ertragsärmsten eines Feldes." 40 Nicht unerheblich ist dagegen der bürokratische Aufwand. Schätzungen beziffern den Verwaltungsaufwand auf ca. $50 \%$ des von der Kommission erstatteten Betrags. ${ }^{41}$ Die Vorschriften sind praktisch auch mit einem so hohen Verwaltungsaufwand nicht kontrollierbar und laden zum Betrug ein. ${ }^{42}$

Ob die Agrarleitlinie eingehalten wird, ist schon von vornherein fraglich, weil für das Überschreiten der Leitlinie keine Konsequenzen beschlossen wurden. Auch früher wurden schon Grenzen für die Agrarausgaben fixiert, die dann nicht eingehalten wurden. So wurde z. B. im Beschluß von Fontainebleau im Jahre 1984 festgelegt, daß der Zuwachs der Agrarausgaben geringer zu sein hat als der Zuwachs an eigenen Mitteln. Dies wurde jedoch in den Folgejahren fast nie erreicht. ${ }^{43}$

37 Vgl. Rat der EG $=$ Stillegung von Ackerflächen $1988=28 \mathrm{ff}$.

38 Vgl. Henze, Zeddies $=$ Reform der Agrarpolitik $=169$.

39 Europäischer Rechnungshof $=$ Jahresbericht $1989=95$.

40 Europäischer Rechnungshof $=$ Jahresbericht $1989=96$.

41 Vgl. Europäischer Rechnungshof $=$ Jahresbericht $1989=96$.

42 Vgl. Schrader $=$ Agrar- und Haushaltspolitik $=30$ und Europäischer Rechnungshof $=$ Jahresbericht $1989=96$.

43 Vgl. Karl-Bräuer-Institut des Bundes der Steuerzahler =EG-Finanzen $=37$. 
Im übrigen ist zu beachten, daß Teile der Agrarausgaben nicht unter die Agrarleitlinie fallen und insofern die Agrarausgaben im Haushalt z. T. verschleiert werden. Der Abbau der gegenwärtigen Lagerbestände wird außerhalb der Leitlinie finanziert. Nicht zur Agrarleitlinie gezählt werden auch die Mittel der Währungsrisikoreserve. ${ }^{44}$ Zudem wurde das Zahlungsziel für Rückerstattungen an die nationalen Haushalte um einen halben Monat verlängert. 45

Die Ziele des Europäischen Rates zur Haushaltsdisziplin verlieren außerdem ihre Wirksamkeit, wenn von der Kommission im Haushalt Manipulationen an den Agrarausgaben vorgenommen werden. So wurden Agrarausgaben auf spätere Haushaltsjahre verschoben, die eigentlich zu Lasten der Haushaltsjahre hätten verbucht werden müssen, in denen sie angefallen waren. 1987 wurde beispielsweise die Dauer des Wirtschaftsjahres auf etwa 10 Monate verkürzt. Dadurch wurde es der Gemeinschaft möglich, 1987 angefallene Ausgaben des Europäischen Ausgleichs- und Garantiefonds für die Landwirtschaft, Abteilung Garantie, für etwa zwei Monate mit zeitlicher Verschiebung im Haushaltsplan auszuweisen. ${ }^{46}$ Auch das Wirtschaftsjahr 1988 wurde - übrigens entgegen einer Stellungnahme des Europäischen Rechnungshofes - für die Abteilung Garantie des Agrarfonds auf 11,5 Monate verkürzt. Außerdem werden sogenannte "negative" Ausgaben - wie z. B. die Milchabgabe - von den Ausgaben abgezogen, anstatt sie auf der Einnahmenseite anzusetzen. Damit werden die Ausgaben zu niedrig ausgewiesen. ${ }^{47}$ Die sich in den Jahren 1988 und 1989 im Haushalt zeigende Eindämmung der Agrarausgaben ist insofern nicht aussagekräftig. Dazu kommt, daß die Entwicklung der Weltmarktpreise in diesen Jahren zu einer Entlastung des Haushaltes geführt hat, die nicht auf einer Ver-

44 Diese Rücklagen werden als vorläufige Mittel im Haushalt eingesetzt, um auf Entwicklungen, die sich bei erheblichen und unvorhergesehenen Schwankungen des auf dem Markt festgestellten Wechselkurses zwischen US-Dollar und ECU gegenüber der im Haushaltsplan veranschlagten Parität ergeben, reagieren zu können. Falls sich die Parität US-Dollar/ECU günstig entwickelt, werden die Einsparungen der Abteilung Garantie bis zu einer Milliarde ECU in die Rücklagen eingestellt. Ist das Gegenteil der Fall, so werden Mittel aus den Rücklagen in die von dieser Entwicklung betroffenen Haushaltslinien der Abteilung Garantie übertragen. Vgl. Rat der EG = Haushaltsdisziplin $1988=29$.

45 Dies entlastet den EG-Haushalt um ca. 1,2 Mrd. ECU pro Jahr. Vgl. Koester, Terwitte $=$ Politikversagen $=130$.

46 Vgl. Europäischer Rechnungshof = Jahresbericht $1987=11$.

47 Vgl. Europäischer Rechnungshof $=$ Jahresbericht $1987=10$. 
besserung der Agrarpolitik beruht. ${ }^{48}$ Für die Jahre 1990 und 1991 ist wieder ein kräftiges Anwachsen der Agrarausgaben zu verzeichnen. 49

Insgesamt hat sich die verzerrte Ausgabenstruktur der Europäischen Gemeinschaften nicht wesentlich verändert. Die Agrarmarktausgaben dominieren mit ungefähr zwei Dritteln nach wie vor. Eine Umstrukturierung der Ausgaben zugunsten anderer Aufgabenbereiche ist nicht festzustellen, vielmehr können höhere Ausgaben für diese Aufgaben nur über eine Erhöhung des Haushaltsvolumens finanziert werden. Andererseits müssen dem immer weiter steigenden Haushalt Grenzen gesetzt werden. Die Begrenzung des Haushalts war nicht zuletzt als Instrument gedacht, um Druck auf die Agrarpolitik auszuüben. Damit wächst jedoch die Gefahr, daß nicht genügend Mittel für andere Aufgaben zur Verfügung stehen. Wenn die Europäischen Gemeinschaften Aufgaben übernommen haben, müssen sie auch in der Lage sein, die Ausgaben dafür zu tragen. Wird dies durch die Dominanz der Agrarausgaben verhindert, ist das Konnexitätsprinzip nicht verwirklicht. Eine Reform der Agrarpolitik wäre daher im Hinblick auf die gesamte föderative Struktur der Europäischen Gemeinschaften notwendig.

\section{Entlastungen von Bund und Ländern durch die Agrarmarktausgaben der Europäischen Gemeinschaften?}

Nach dem Konnexitätsprinzip müßte es mit zunehmendem finanziellem Engagement der Europäischen Gemeinschaften in der Agrarpolitik zu Kürzungen nationaler Agrarausgaben kommen. Dies gilt vor allem dann, wenn die Gemeinschaft nicht mehr nur die nationale Politik unterstützt, sondern - wie bei der Agrarmarktpolitik - eine eigenständige Politik betreibt. ${ }^{50}$ Ob bei Bund und Ländern tatsächlich Entlastungen vorliegen, soll im folgenden geprüft werden. Eine quantitativ exakte Erfassung möglicher Entlastungswirkungen ist allerdings nicht möglich, da ja nicht bekannt ist, wie groß der Umfang der

48 Vgl. dazu auch Sachverständigenrat zur Begutachtung der gesamtwirtschaftlichen Entwicklung = Jahresgutachten 1990/91 = Ziff. 481 .

49 Die Kommission schätzte im Frühjahr 1991, daß ohne weitere Korrekturmaßnahmen die Ausgaben des EAGFL-Garantie 1991 gegenüber dem Vorjahr um 32\% steigen würden. Die Agrarleitlinie würde nach diesen Schätzungen um 480 Mio. ECU überschritten. Vgl. Kommission der EG =Agrarpreise 1991/92=6.

$50 \quad$ Vgl. Schüler $=$ Die vierte Ebene $=48$. 
Agrarausgaben von Bund und Ländern wäre, wenn es die Agrarmarktordnungspolitik nicht gäbe. Es muß also bei Tendenzaussagen bleiben.

Ein weiteres Problem ergibt sich dadurch, daß nicht nur die Agrarmarktpolitik betrachtet werden darf. Die gemeinsame Preis- und Einkommenspolitik für die Landwirtschaft hatte schon bald nach der Gründung der gemeinsamen Agrarmarktorganisation aufgrund der stark divergierenden Strukturen der Landwirtschaft von Mitgliedstaat zu Mitgliedstaat und der unterschiedlichen wirtschaftlichen Entwicklung zu Konflikten mit den nationalen Zielen der Agrarpolitik gefuihrt und starke nationale Aktivitäten in den Bereichen der Agrarpolitik ausgelöst, in denen die nationale Autonomie nicht oder nicht wesentlich eingeschränkt worden war. "Dies waren vor allem die Strukturpolitik, die Sozialpolitik und auch die Steuerpolitik für den landwirtschaftlichen Sektor ..."51 Um ein komplettes Bild zu erhalten, müssen auch diese Bereiche mit in die Betrachtung einbezogen werden.

Die Marktordnungsausgaben, die dem Bund von den Europäischen Gemeinschaften erstattet werden, weisen mit geringen Schwankungen eine stark steigende Tendenz auf. Betrachtet man die Ausgaben des Bundes für Ernährung, Landwirtschaft und Forsten, die im Finanzbericht ausgewiesen werden, so ist zunächst tatsächlich ein Rückgang der Ausgaben bis zum Jahre 1976 festzustellen. Danach stiegen die Ausgaben, allerdings mit einigen Schwankungen, wieder an. Vor allem in den letzten Jahren lassen sich deutliche Steigerungen ablesen (siehe Tabelle 23). Auch bei den Ausgaben der Länder läßt sich kein Entlastungseffekt feststellen. Ihre Ausgaben stiegen insgesamt ebenfalls mit einigen Schwankungen an.

Ein Entlastungseffekt ist noch weniger gegeben, wenn man neben den Ausgaben auch die Steuervergünstigungen für die Landwirtschaft gemäß den Subventionsberichten der Bundesregierung betrachtet, die nach der Einführung der Agrarmarktordnung keineswegs zurückgingen. Vor allem seit 1985 sind sie wieder sehr hoch und übertreffen sogar die direkten Ausgaben für Ernährung, Landwirtschaft und Forsten. Die starke Erhöhung der Steuervergünstigungen im Jahre 1985 hat ihre Ursache in dem nationalen Einkommensausgleich für die fünfprozentige Preissenkung infolge der DM-Aufwertung im Rahmen des Europäischen Währungssystems, der über das Mehrwertsteuersystem abgewikkelt wird. Dies zeigt, daß Bund und Länder keine Gelegenheit ungenutzt gelas-

51 Thoroe = Europäische Agrarpolitik = 114. 
Tabelle 23: Erstattungen für Marktordnungsausgaben und nationale Agrarausgaben in der Bundesrepublik Deutschland in den Jahren 1970 bis 1990 (in Mio. DM)

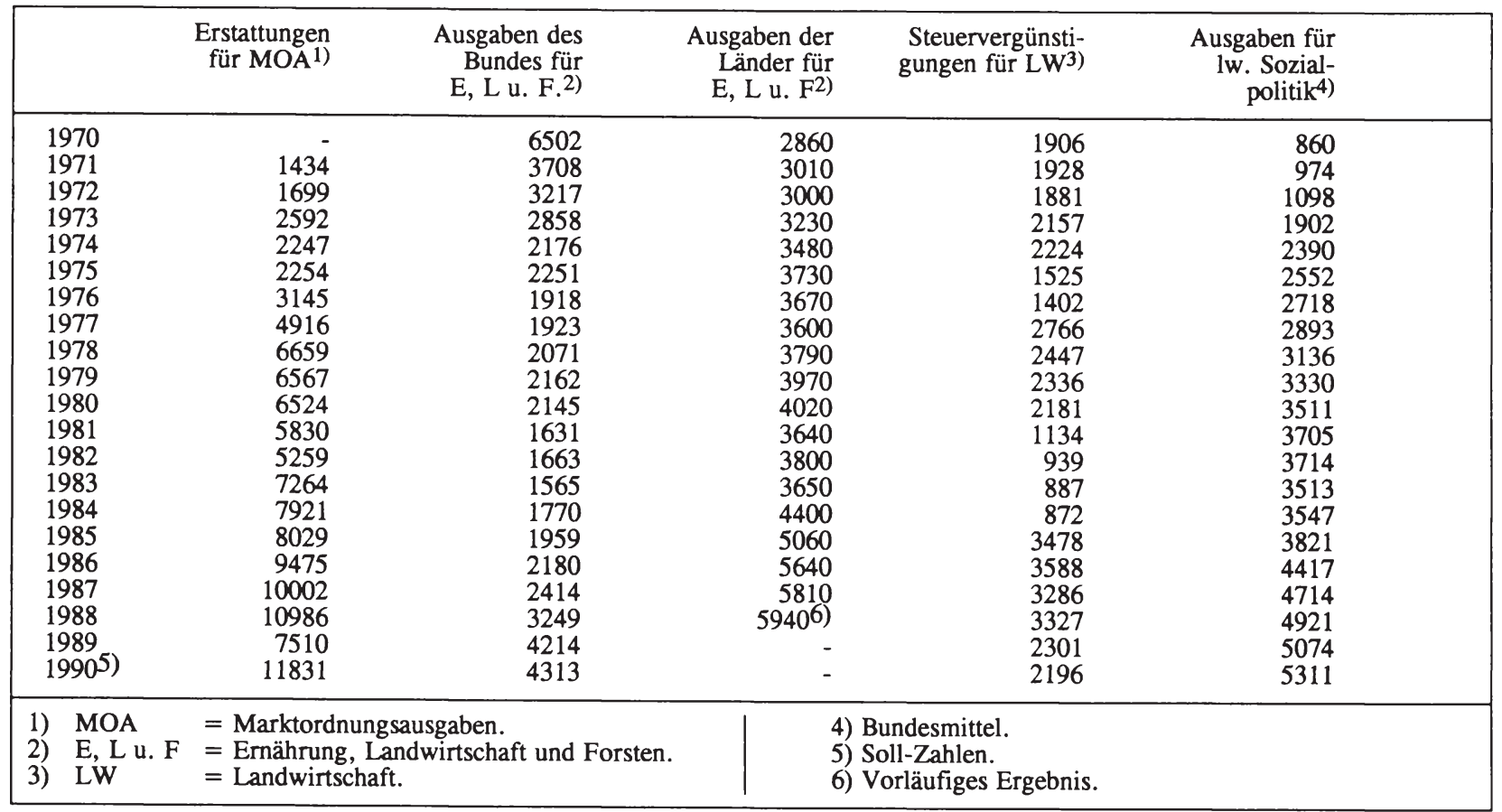

Quelle: Bundesministerium der Finanzen =Finanzberichte der Jahre $1972-1990=$ sowie Subventionsberichte 9 bis 12 und Statistisches Bundesamt $=$ Konten und Standardtabellen $=283$ sowie $=$ Revidierte Ergebnisse $=$ 210 und 213. 
sen haben, um Preissenkungen der Europäischen Gemeinschaften durch nationale Programme zu kompensieren. 52

Dieser Eindruck bestätigt sich auch, wenn man die Agrarsozialpolitik betrachtet. Diese hat sich zu einem der wichtigsten Bereiche der nationalen Agrarpolitik entwickelt. ${ }^{53}$ Die Ausgaben für die landwirtschaftliche Sozialpolitik stiegen sehr stark von 860 Mio. DM (1970) auf 5.311 Mio. DM (1990).

Insgesamt sind die Entlastungswirkungen der europäischen Agrarpolitik eher fraglich. Die nationalen Belastungen durch die Landwirtschaft haben insgesamt - verstärkt gilt dies seit Mitte der achtziger Jahre - zugenommen, ohne daß auf Gemeinschaftsebene ein Abbau der Haushaltsbelastung erkennbar wird. ${ }^{54}$

\section{Zusätzliche Ausgaben bei Bund und Ländern durch Beschlüsse der Europäischen Gemeinschaften}

Bei der Betrachtung der Ausgabenverteilung muß noch ein weiterer Aspekt berücksichtigt werden: Durch eine auf Gemeinschaftsebene beschlossene Politik können zusätzliche Belastungen auf den Bund, aber auch auf die Länder zukommen. ${ }^{55}$ Man kann hier in Anlehnung an Peffekoven von einem sogenannten unsichtbaren Finanzausgleich sprechen, bei dem eine Körperschaft bei der Wahrnehmung einer ihr zugewiesenen Aufgabenkompetenz bewußt oder unbewußt andere Körperschaften belastet. 56

Besonders bei der Agrarpolitik führten Gemeinschaftsbeschlüsse immer wieder zu zusätzlichen Ausgaben von Bund und Ländern. Die Beschlüsse des Europäischen Rates vom 11. und 13.2.1988, die u. a. die Mitgliedstaaten verpflichteten, den Landwirten Maßnahmen zur Flächenstillegung, Extensivierung und Umstellung der Erzeugung gegen Einkommensausgleich anzubieten ${ }^{57}$, wurden in der Bundesrepublik Deutschland im Rahmen eines Sonderrahmenplans in der Gemeinschaftsaufgabe "Verbesserung der Agrarstruktur und des Küstenschutzes" umgesetzt. Der Sonderrahmenplan wurde für den Zeitraum 1988

52 Vgl. dazu auch Schrader $=$ Agrar- und Haushaltspolitik $=8$.

53 Vgl. Scheele $=$ Sektorale Einkommensübertragungen $=203$.

54 So auch Schrader $=$ Agrar- und Haushaltspolitik $=8 \mathrm{f}$.

55 Vgl. Schüler $=$ Die vierte Ebene $=49$.

56 Vgl. Peffekoven $=$ Finanzausgleich $\mathrm{I}=618$.

57 Vgl. $=$ Agrarbericht $1989=75$. 
bis 1993 aufgestellt. Er sieht eine finanzielle Beteiligung von Bund und Ländern im Verhältnis 70:30 vor. ${ }^{58}$ Dabei wurden 1989 Bundesmittel in Höhe von 200 Mio. DM und Ländermittel in Höhe von 86 Mio. DM und in den Folgejahren Bundesmittel in Höhe von 250 Mio. DM und Ländermittel in Höhe von 107 Mio. DM veranschlagt. Die Verwaltung der Regelung und die Leistung der Zahlung ist Sache der Länder. "Das Landwirtschaftsministerium in Bonn koordiniert die Durchführung der Regelung, kann diese jedoch nicht unmit-telbar beeinflußen. Die Verwaltungspraxis unterscheidet sich von Land zu Land ..."59

Auch die im April 1988 vom Ministerrat verabschiedete "Verordnung zur Einführung einer Gemeinschaftsregelung zur Förderung der Einstellung der landwirtschaftlichen Erwerbstätigkeit" führte zu Mehrausgaben auf nationaler Ebene, die in diesem Fall allein zu Lasten des Bundes gehen. Die Umsetzung dieser Verordnung kostet den Bund 115 Mio. DM 1989, 250 Mio. DM 1990, 350 Mio. DM 1991 und 380 Mio. DM $1992 .{ }^{60}$

Während bei den bisher erwähnten Programmen der Agrarpolitik die Kosten für Bund und/oder Länder sehr genau angegeben werden können, ist eine exakte Quantifizierung bei anderen Zusatzlasten, etwa bei der Umsetzung der Richtlinie über die schulische Betreuung der Kinder von Wanderarbeitern oder bei Richtlinien zum Gewässerschutz, nicht immer möglich. Insgesamt läßt sich daher nicht abschätzen, wie hoçh die Zusatzlast durch Beschlüsse der Gemeinschaft ist.

Grundsätzlich gibt es keine allgemeine rechtliche Klärung der Frage, wer auf nationaler Ebene die Lasten der Gemeinschaftsmaßnahmen zu tragen hat. Bei agrarstrukturpolitischen Maßnahmen sprachen sich die Agrarminister von Bund und Ländern dafür aus, bis zu einer Verfassungsregelung dieser Frage die Maßnahmen zur Verbesserung der Agrarstruktur und des Küstenschutzes vorläufig auch dann wie Gemeinschaftsaufgaben nach Art. 91a GG auszuführen und zu finanzieren, wenn sie durch Rechtsakte der Europäischen Gemeinschaften geregelt worden sind. Obwohl die Agrarminister festgestellt haben, daß ein Planungsspielraum ein wesentliches Kriterium des Art. 91a GG ist, sollte diese vorläufige Gleichstellung auch dann gelten, wenn die

58 Vgl. =Agrarbericht $1989=72$.

59 Vgl. Europäischer Rechnungshof $=$ Jahresbericht $1989=93$.

60 Vgl. $=$ Agrarbericht $1989=73$. 
Gemeinschaftsregelungen keinen Planungsspielraum zur innerstaatlichen Ausgestaltung der Maßnahme belassen. ${ }^{61}$ Einzig entscheidendes Kriterium war damit nur noch, ob es sich um eine Verbesserung der Agrarstruktur handelt; dieser Nachweis läßt sich angesichts des Maßnahmenkatalogs leicht führen. Bei der Diskussion um die Finanzierungsfrage wurde immer wieder von den Ländern betont, daß sie Maßnahmen nicht allein finanzieren wollten, um deutlich zu machen, daß der Bund nicht einfach in Brüssel zu ihren Lasten beschließen könne. Auch in der Finanzierung sollte deutlich sein, wer die Verantwortung für den Beschluß der Maßnahme trägt. ${ }^{62}$

\section{Die Problematik der Mischfinanzierung - das Beispiel der Struktur- fonds}

In vielen Politikbereichen beteiligen sich die Europäischen Gemeinschaften zu einem bestimmten Teil an den nationalen Ausgaben, so daß eine Mischfinanzierung durch die Europäischen Gemeinschaften und die Mitgliedstaaten vorliegt. Dies gilt für die Ausgaben im Rahmen der Entwicklungshilfe, der Verkehrspolitik, der Energiepolitik sowie für die Technologiepolitik. ${ }^{63}$ Die Mischfinanzierung ist inzwischen auch auf die Agrarpolitik ausgedehnt worden, wie das Flächenstillegungsprogramm zeigt. An diesem Programm beteiligen sich die Europäischen Gemeinschaften mit Sätzen zwischen 15 und 50\% an den Beihilfen, die ansonsten von Bund und Ländern getragen werden.

Das markanteste Beispiel sind jedoch die Ausgaben der Strukturfonds. Die Gemeinschaft unterstützt hier Projekte in den Mitgliedstaaten mit bestimmten Sätzen. Für Maßnahmen in den Regionen, die für die Interventionen im Rahmen des Ziels Nr. 1 in Betracht kommen, liegen diese Sätze bei höchstens $75 \%$ der Gesamtkosten und bei mindestens $50 \%$ der öffentlichen Ausgaben. Für Maßnahmen in den übrigen Regionen sind Sätze in Höhe von höchstens $50 \%$ der Gesamtkosten und mindestens $25 \%$ der öffentlichen Ausgaben angesetzt. ${ }^{64}$

61 Vgl. Blaschke $=$ Gemeinschaftsaufgabe und EG-Politik $=46$.

62 Vgl. Blaschke $=$ Gemeinschaftsaufgabe und EG-Politik $=46$.

63 Vgl. dazu auch Karl-Bräuer-Institut des Bundes der Steuerzahler =EG-Finanzen $=20$.

64 Vgl. Rat der EG = Aufgaben und Effizienz der Strukturfonds 1988=17. 
Mischfinanzierungen weisen eine ganze Reihe von Nachteilen auf. Wenn nationale oder regionale Stellen nur für einen Teil der Projektkosten aufkommen müssen, werden die Anreize für eine sparsame Ressourcenverwendung gemindert. Der Investitionskalkül wird systematisch verfälscht, wenn dem Nutzen aus dem Projekt vor Ort nur ein Teil der Kosten gegenübergestellt wird, und es besteht die Gefahr, daß über den eigentlichen Bedarf investiert wird. Weiter besteht die Gefahr einer Fehlallokation dadurch, daß weniger dringliche Vorhaben zuerst in Angriff genommen werden oder sogar abseits wirklichen Bedarfs Vorhaben geplant werden, nur um an Fördermittel heranzukommen. So standen u. a. agrarstrukturpolitische Entscheidungen der Länder häufig unter der besonderen Frage, ob sie die Tatsache, daß Erstattungen aus Brüssel fließen, negativ beeinflussen können. ${ }^{65}$

Bei der Vielzahl von Einzelprogrammen und entsprechenden Bewilligungsrichtlinien hängt die Bewilligung von Mitteln vom Informationsstand sowohl der Antragsteller als auch der Kommissions-Beamten über die Region ab. Der Kenntnisstand über die Möglichkeiten der Förderung kann in den Mitgliedstaaten unterschiedlich sein. Wichtig sind in diesem Zusammenhang auch informelle Kontakte zwischen den nationalen Regierungen und der Kommission. Bei der Beurteilung durch die Kommission kann nicht immer davon ausgegangen werden, daß "fernab von den Problemen"66 in allen Bereichen ein einheitlicher Informationsstand besteht.

65 Vgl. Blaschke $=$ Gemeinschaftsaufgabe und EG-Politik $=45$.

66 Karl-Bräuer-Institut des Bundes der Steuerzahler $=$ EG-Finanzen $=75$. 
Tabelle 24: Aufgliederung der EFRE-Mittel auf die Länder zwischen 1975 und 1988

\begin{tabular}{|lrr|}
\hline & $\begin{array}{r}\text { absolute Zuflüsse } \\
\text { in Mio. ECU }\end{array}$ & $\begin{array}{r}\text { Zuschüsse pro Kopf der } \\
\text { Bevölkerung in den } \\
\text { Fördergebieten } \\
\text { in ECU/Einwohner }\end{array}$ \\
Baden-Württemberg & & \\
Bayern & 20,86 & 210 \\
Berlin & 198,31 & 57 \\
Bremen & 67,09 & 37 \\
Hamburg & 8,21 & 12 \\
Hessen & - & - \\
Niedersachsen & 54,31 & 38 \\
Nordrhein-Westfalen & 189,94 & 35 \\
Rheinland-Pfalz & 102,92 & 21 \\
Saarland & 76,80 & 48 \\
Schleswig-Holstein & 102,47 & 99 \\
\hline
\end{tabular}

Quelle: Kommission der EG =EFRE-Jahresbericht $1988=62$ und 100.

Schließlich birgt die Tatsache, daß von den nationalen Empfängerhaushalten die Aufbringung eines Eigenanteils gefordert wird und Projekte häufig vorfinanziert werden müssen, die Gefahr in sich, daß vorwiegend bereits entwikkelte Regionen weiterentwickelt werden. ${ }^{67}$ Insofern können Zuweisungen mit Eigenbeteiligungsauflagen strukturkonservierend wirken. ${ }^{68}$ In Tabelle 24 sind die Mittel des Europäischen Fonds für regionale Entwicklung, die zwischen 1975 und 1988 den einzelnen Ländern zugute kamen, aufgeschlüsselt. Der absolut größte Betrag fließt dabei nach Bayern, gefolgt von Niedersachsen und Schleswig-Holstein. Dies erscheint angesichts der Größe dieser Länder und ihrer strukturellen Probleme nicht überraschend. Betrachtet man dagegen die $\mathrm{Zu}$ flüsse pro Kopf der Bevölkerung in den ausgewiesenen Fördergebieten, so zeigt sich ein anderes Bild. Hier liegt das Land Baden-Württemberg mit Abstand an der Spitze. Daß ein Land, das im nationalen Finanzausgleich der größte "Zahler" ist, den größten Betrag an Zuschüssen pro Kopf der Bevölkerung in den Fördergebieten erhielt, scheint die obengenannten Befürchtungen zu bestätigen.

67 Vgl. Petersen $=$ Agrarpolitik $=36$.

68 Vgl. dazu auch Hansmeyer $=$ Zweckzuweisungen $=438 \mathrm{f}$. 
Vor der Reform der Strukturfonds war es die Regel, daß die Ausgaben für strukturelle Maßnahmen zunächst von den Mitgliedstaaten bestritten wurden und die Europäischen Gemeinschaften rückwirkend Erstattungen gewährten. Es konnte jedoch von vornherein nicht sicher mit den Erstattungen gerechnet werden, da ein großer Teil der Anträge auf Erstattungen abgelehnt wurde. ${ }^{69}$ Hier liegt die Vermutung nahe, daß die Projekte auch ohne Gemeinschaftshilfen durchgeführt worden wären und daß hier lediglich Mitnahmeeffekte vorliegen. ${ }^{70}$ Auch nach der Reform der Strukturfonds stellen die Gelder der Europäischen Gemeinschaften für die Empfänger aufgrund der Zeitverzögerung beim Bewilligungsverfahren oftmals de facto eine Erstattung bereits erfolgter Ausgaben dar. ${ }^{71}$

Weitere Probleme im Rahmen der Mischfinanzierung ergeben sich dadurch, daß häufig die Folgekosten von Projekten nicht berücksichtigt werden. So wurden beispielsweise in Sizilien mit Hilfe des Regionalfonds verschiedene Wasserversorgungsanlagen gebaut, die nicht in Betrieb genommen werden können, weil den nationalen Stellen die Finanzierungsmittel für den laufenden Betrieb fehlen. ${ }^{72}$

Schließlich ist zu den Mischfinanzierungen kritisch anzumerken, daß sie den Entscheidungsspielraum der Parlamente einschränken; denn an den entsprechenden (Vor-)Entscheidungen der Exekutive bezüglich Mischfinanzierungen können von den Parlamenten in der Regel keine Änderungen vorgenommen werden. ${ }^{73}$

691985 wurden beispielsweise von 387 Anträgen der Bundesrepublik 217 angenommen, vgl. Kommission der EG =EFRE-Jahresbericht $1985=42$.

70 Vgl. Karl-Bräuer-Institut des Bundes der Steuerzahler =EG-Finanzen $=72$.

71 So auch Europäischer Rechnungshof =Jahresbericht $1989=124$.

72 Vgl. dazu Europäischer Rechnungshof = Jahresbericht $1987=101$.

73 Vgl. Brümmerhoff $=$ Finanzwissenschaft $=505 \mathrm{f}$. 


\section{Einnahmenautonomie}

\section{Die Einnahmenautonomie}

Bei der Einnahmenverteilung stellt sich insbesondere die Frage nach einer ausreichenden Einnahmenautonomie der einzelnen Ebenen. Zunächst soll den Problemen, die sich für die nationalen Ebenen durch die Anpassung an die wachsenden Einnahmenkompetenzen der Europäischen Gemeinschaften ergeben, nachgegangen werden. Auf der Gemeinschaftsebene ist vor allem die Frage nach dem Zusammenhang zwischen den zur Aufgabenerfüllung notwendigen Ausgaben und den Einnahmen, aber auch nach der Rückkopplung zwischen der Belastung der Bürger mit Abgaben und den dadurch finanzierten Ausgaben zu stellen.

\section{Die Einnahmenautonomie von Bund und Ländern}

\section{a) Die Anpassung der nationalen Haushalte an den Finanzbedarf der Europäischen Gemeinschaften}

Bei der Anpassung der nationalen Haushalte an den Finanzbedarf der Europäischen Gemeinschaften sind mehrere Aspekte zu unterscheiden, die grundsätzlich für alle Mitgliedstaaten gelten. Wenn Aufgaben, die bisher national erfüllt wurden, von den Europäischen Gemeinschaften übernommen werden und diese auch die erforderlichen Ausgaben tragen, können die nationalen Haushalte ihre eigenen Ausgaben im entsprechenden Ausmaß reduzieren. Dadurch benötigen sie auch weniger Einnahmen. Diese "freiwerdenden" Einnahmen können an die Europäischen Gemeinschaften abgeführt werden. Henke spricht hier von "Vergemeinschaftung der Mittel" und von einem "Struktureffekt nach Popitz"1. Damit ist gemeint, daß sich vor allem die Verteilung der Ausgaben auf die einzelnen Ebenen zugunsten des übergeordneten Haushalts verschiebt entsprechend Popitz' "Gesetz von der Anziehungskraft des übergeordneten Haushalts"2. Dabei muß nicht von einer Konstanz der Ausgabenvolumen ausgegangen werden. Wenn eine Aufgabe auf die Gemeinschaftsebene verlagert wurde, weil sie dort effizienter gelöst werden kann, etwa durch die Ausnut-

$1 \quad$ Henke $=$ Finanzierung der $E G=26$.

$2 \quad$ Popitz $=$ Finanzausgleich $=348 \mathrm{f}$. 
zung von economies of scale, müßte es eigentlich zu einer Verminderung des Gesamtvolumens der Ausgaben kommen.

$\mathrm{Da} ß$ die gesamten öffentlichen Ausgaben in der Europäischen Gemeinschaft nicht gesunken sind, liegt erstens daran, daß sie bestimmte Aufgaben eben doch nicht effizienter löst. ${ }^{3}$ Zweitens erfüllt sie zusätzlich neue Aufgaben, die sich so nur der Gemeinschaft stellen, und drittens wird sie auch ergänzend zu den Mitgliedstaaten tätig. In diesen Fällen ist eine Reduzierung der nationalen Ausgaben nicht vorgesehen. ${ }^{4}$ Das bedeutet, daß den Europäischen Gemeinschaften zusätzlich Mittel bereitgestellt werden müssen. Henke spricht hier von "Zusatzfinanzierung" und von einem "Wagner-Impuls"5, der die Ausdehnung der öffentlichen Tätigkeit insgesamt charakterisieren soll. Für die Bürger bedeutet dies eine höhere Steuerbelastung.

\section{b) Wirkungen auf die nationale Föderalismusstruktur in der Bundes- republik Deutschland}

Der Anpassungsprozeß hat auch Auswirkungen auf die nationale Föderalismusstruktur, denn es stellt sich die Frage, wie die entstehenden Lasten auf die nationalen Ebenen verteilt werden sollen. Je höher die Finanzierungslast, desto schwieriger wird die nationale Anpassung. Diese Probleme nehmen noch zu, wenn Einnahmenregelungen von der Gemeinschaft getroffen werden, die in die nationale Steueraufteilung zwischen den Gebietskörperschaften einwirken, wie dies in der Bundesrepublik Deutschland für die Mehrwertsteuer de facto der Fall ist. Berücksichtigt man, daß die nationalen Föderalismusstrukturen durch die Übertragung von Aufgabenkompetenzen und Ausgabenkompetenzen an die Europäischen Gemeinschaften verändert wurden, so kann eine geänderte Verteilung der Finanzierungslasten auf die verschiedenen staatlichen Ebenen - entsprechend den Entlastungseffekten der betreffenden Ebene durch die von den Europäischen Gemeinschaften übernommenen Aufgaben - theoretisch zwar durchaus sinnvoll sein. Allerdings ist zu vermuten, daß in der Realität die zu-

3 Hier sei noch einmal auf die bürokratischen Probleme verwiesen.

4 Das schließt nicht aus, daß in der Realität die Mittel der Europäischen Gemeinschaften nicht zusätzlich, sondern als Ersatz nationaler Mittel eingesetzt werden. Zur Frage der Zusätzlichkeit der Mittel des Europäischen Fonds für regionale Entwicklung vgl. Bock $=$ Instrumente des Finanzausgleichs $=58 \mathrm{ff}$.

$5 \quad$ Henke $=$ Finanzierung der $\mathrm{EG}=26$. 


\section{Einnahmenautonomie}

sätzlichen Auseinandersetzungen im föderativen System der Mitgliedstaaten als Folge von Überlegungen über Einnahmen der Europäischen Gemeinschaften nicht als Chance für eine Neuordnung ergriffen werden. ${ }^{6}$

In der Bundesrepublik Deutschland trägt bisher ausschließlich der Bund die unmittelbaren Lasten aus der Abtretung von Einnahmequellen an die Europäischen Gemeinschaften. Die Länder begründen dies mit dem Hinweis auf die traditionelle zentralstaatliche Zuständigkeit in der Außenpolitik und auf die fehlenden Einflußmöglichkeiten der Länder auf die Gemeinschaftspolitik. Andererseits profitieren jedoch nicht nur der Bund von den Ausgaben der Gemeinschaft, sondern auch die Länder, so daß eine Beteiligung der Länder nicht völlig aus den Überlegungen ausgeschlossen werden sollte. ${ }^{7}$ Es besteht allerdings die Möglichkeit, daß bei einer weiter steigenden Belastung durch Abführungen an die Europäischen Gemeinschaften dieser Aspekt bei den Verhandlungen über die innerstaatliche Verteilung des Mehrwertsteueraufkommens an Bedeutung gewinnt und damit die Länder mittelbar doch an den Belastungen beteiligt werden.

Betrachtet man die Anteile der Europäischen Gemeinschaft, des Bundes, der Länder und der Gemeinden am bundesdeutschen Steueraufkommen (siehe Tabelle 25), so ließ sich zu Beginn der achtziger Jahre ein Rückgang des Anteils des Bundes feststellen. Dies wird vom Bundesministerium der Finanzen u. a. ${ }^{8}$ damit begründet, "daß die Abführung der EG-Mehrwertsteuereigenmittel bei ständig steigender Bedeutung allein zu Lasten des Bundes geht ..."

6 Vgl. dazu Caesar $=$ Neue Steuern für die $E G=82$.

7 Vgl. dazu auch Schüler $=$ Die vierte Ebene $=48$.

8 Ein weiterer Grund für den Rückgang des Bundesanteils sieht das Finanzministerium in dem für den Bund ungünstig hohen Anteil an unterdurchschnittlich wachsenden Steuern, insbesondere der Umsatzsteuer und den Verbrauchsteuern. Vgl. Bundesministerium der Finanzen $=$ Finanzbericht $1990=82$.

9 Bundesministerium der Finanzen $=$ Finanzbericht $1990=82$. 
Tabelle 25: Die Anteile von Bund, Ländern, Gemeinden und Europäischen Gemeinschaften am bundesdeutschen Steueraufkommen

\begin{tabular}{|ccccc|}
\hline \multicolumn{5}{c|}{ Anteile am Gesamtaufkommen } \\
in \% \\
Länder Gemeinden 1) & EG \\
1980 & Bund & 34,8 & 14,0 & 2,9 \\
1981 & 48,3 & 34,5 & 13,5 & 3,3 \\
1982 & 48,7 & 34,8 & 13,5 & 3,3 \\
1983 & 48,4 & 35,0 & 13,6 & 3,5 \\
1984 & 48,0 & 35,1 & 13,8 & 3,6 \\
1985 & 47,6 & 35,3 & 14,1 & 3,5 \\
1986 & 47,2 & 35,8 & 14,1 & 4,0 \\
1987 & 46,2 & 35,9 & 13,9 & 3,9 \\
1988 & 46,3 & 35,9 & 14,1 & 4,8 \\
1989 & 45,1 & 35,8 & 13,8 & 4,3 \\
$\left.1990^{2}\right)$ & 46,1 & 35,3 & 13,7 & 3,9 \\
\hline 1) Einschließlich Gemeindesteuern der Stadtstaaten. \\
2) Bundesgebiet entsprechend dem Stand vor dem 3.10.1990. \\
\hline
\end{tabular}

Quelle: Bundesministerium der Finanzen $=$ Finanzbericht $1990=83$.

\section{c) Nicht-fiskalische Anpassungsprobleme}

Schließlich sind auch noch nicht-fiskalische Folgen des Anpassungsprozesses zu berücksichtigen. ${ }^{10}$ Die Einschränkung der nationalen Haushaltsautonomie durch Ausgabenbeschränkungen oder Einnahmenausfälle, aber auch durch die Harmonisierungsvorschriften führt dann zu Problemen, wenn nationale Eingriffsmöglichkeiten entfallen und diese Handlungspotentiale von den Europäischen Gemeinschaften nicht oder in anderer als der nationalen Auffassung entsprechenden Weise ausgefüllt werden. Verlieren die nationalen Ebenen beispielsweise die Ertragshoheit über bestimmte Steuern, so werden zahlreiche Interventionsmöglichkeiten beschnitten. Unterbleibt eine entsprechende Funktionsübernahme seitens der Europäischen Gemeinschaften, kann es zu einer Überfrachtung der im nationalen Besitz verbleibenden finanzpolitischen Ströme mit den verschiedensten Anforderungen kommen, die im übrigen eine später erforderliche Harmonisierung noch zusätzlich erschweren kann. 11

10 Vgl. dazu Henke =Finanzierung der $E G=27$.

11 Vgl. Henke =Finanzierung der $E G=27$. 


\section{Einnahmenautonomie}

\section{Die Einnahmenautonomie der Gemeinschaft}

\section{a) Die begrenzten Einnahmenkompetenzen der Europäischen Gemein- schaften}

Während die Europäische Gemeinschaft für Kohle und Stahl von Beginn an durch ihre Eigenmittel über eine ausreichende Einnahmenautonomie verfügte, war die Einnahmenautonomie der Europäischen Wirtschaftsgemeinschaft und der Europäischen Atomgemeinschaft vor dem Eigenmittelbeschluß von 1970 sehr begrenzt; die Gemeinschaften erhielten nur Finanzbeiträge von den Mitgliedstaaten. Das Finanzierungssystem dieser Gemeinschaften stellte damals ein reines Zuweisungssystem dar. Zur Unterscheidung des neuen Finanzierungssystems vom System der Finanzbeiträge wurde der Begriff "eigene Mittel" (Art. 201 EWGV) eingeführt. Inwieweit das System der Eigenmittel die Haushaltsautonomie der Gemeinschaft besser gewährleistet, soll im folgenden geprüft werden.

\section{aa) Die Einnahmenkompetenzen bei den "originären" Eigenmitteln}

Bei den Agrarabschöpfungen und den Zöllen besitzt die Gemeinschaft die alleinige Objekt- und Ertragshoheit. Diese Eigenmittelarten sind dem Trennsystem zuzuordnen. ${ }^{12}$ Die Einnahmenautonomie ist daher bei den Zöllen und den Agrarabschöpfungen prinzipiell sehr groß; sie wird allerdings dadurch etwas eingeschränkt, daß diese Eigenmittel primär zum Schutz der gewerblichen Wirtschaft und der landwirtschaftlichen Produktion erhoben werden. "Deshalb kann die Höhe der Einnahmen aus diesen Abgaben nicht nach haushaltspolitischen Erfordernissen variiert werden, für die Festlegung der Abgabensätze sind vielmehr in erster Linie handels- und agrarpolitische Gesichtspunkte maßgeblich." 13

12 "Auch wenn die Mitgliedstaaten nach dem Eigenmittelbeschluß von 1988 nur $90 \mathrm{vH}$ des Aufkommens bei Agrarabschöpfungen und Zöllen an die Europäischen Gemeinschaften abführen und $10 \mathrm{vH}$ als Pauschalerstattung für Erhebungskosten einbehalten, ändert sich dadurch nicht die Ertragskompetenz. Denn dieses Abwicklungsverfahren, das lediglich aus Gründen der Vereinfachung eingeführt wurde, ist nicht gleichzusetzen mit einem Verbundsystem, bei dem der EG-Ebene $90 \mathrm{vH}$, den Mitgliedstaaten 10 $\mathrm{vH}$ des Aufkommens zustehen. Bei gleichem finanziellen Ergebnis ist der konzeptionelle Ansatz doch unterschiedlich." Messal =EG-Finanzierung = 129f.

13 Nittka $=$ Finanzierungssystem $=209 \mathrm{f}$. 
Außerdem ist zu beachten, daß die Verwaltungshoheit dieser - aber auch der übrigen - Eigenmittel bei den Mitgliedstaaten liegt. Die Bedeutung dieser Tatsache wird häufig unterschätzt. Als wesentlicher Vorteil eigener Mittel wird angeführt, daß sie supranationale Organisationen von den nationalen Regierungen unabhängig machen. Es sei den einzelnen Ländern nicht mehr möglich, Aktivitäten, die sie ablehnen, durch die Verweigerung der Zahlung von Finanzbeiträgen zu verhindern. ${ }^{14}$ Solche Praktiken sind jedoch auch jetzt nicht grundsätzlich auszuschließen, weil die Europäischen Gemeinschaften bei der Erhebung der Eigenmittel auf die Mitwirkung und Unterstützung der nationalen Finanzbehörden angewiesen sind; die Mehrwertsteuer-Eigenmittelzahlungen oder die Bruttosozialprodukt-Eigenmittelzahlungen könnten genauso leicht zurückgehalten werden.

\section{bb) Die Einnahmenkompetenzen bei den Mehrwertsteuer-Eigenmitteln}

Bei der Objekthoheit der Mehrwertsteuer-Eigenmittel wird häufig von einer geteilten Objekthoheit gesprochen. Dies ist nicht korrekt, denn diese geteilte Objekthoheit besteht nur für die nationalen Mehrwertsteuern. Von diesen Überlegungen ist jedoch die Objekthoheit für die Mehrwertsteuer-Eigenmittel der Europäischen Gemeinschaften zu trennen. Bei der Berechnung der fiktiven Bemessungsgrundlage werden nationale Ausnahmeregelungen weitgehend herausgerechnet, so daß hier die Europäischen Gemeinschaften die Objekthoheit fast gänzlich besitzen. ${ }^{15}$ Auch über den abzuführenden Satz bestimmen die Europäischen Gemeinschaften, wobei sie allerdings an die Höchstgrenze von $1,4 \%$ gebunden sind. Diese Grenze, die für jedes einzelne Mitgliedsland gilt, ist in den letzten Jahren stets zum Tragen gekommen. Insofern kann man auch bei den Mehrwertsteuer-Eigenmitteln der Europäischen Gemeinschaften von einer nur begrenzten Objekthoheit sprechen.

Hinsichtlich der Ertragshoheit sei hier nochmals betont, daß es sich bei den Mehrwertsteuer-Eigenmittelsätzen nicht um einen direkten Zuschlag zur nationalen Mehrwertsteuer handelt. Die nationale Belastung durch MehrwertsteuerEigenmittel tritt im Rahmen des gegenwärtigen Mehrwertsteuer-Eigenmittelsystems gegenüber dem Steuerschuldner (Unternehmer) und dem Letztverbrau-

14 Vgl. dazu Peffekoven =Eigene Einnahmen $=321 \mathrm{f}$.

15 Den Mitgliedstaaten bleibt allerdings die Wahl des Berechnungsmodus. Hier haben sich alle Mitgliedstaaten für die Einnahmenmethode und nicht für die Methode anhand der Steuererklärungen entschieden. 


\section{Einnahmenautonomie}

cher nicht unmittelbar in Erscheinung. "Den Mitgliedstaaten ist es freigestellt, ob und wie sie die an die Europäischen Gemeinschaften abzuführenden MWStEigenmittel kompensieren. Durch Änderungen der nationalen MWSt-Sätze soll der Letztverbraucher belastet werden, durch Änderungen des MWSt-Eigenmittelsatzes dagegen - unmittelbar nur - der Mitgliedstaat." 16 Eine direkte Beteiligung der Europäischen Gemeinschaften an den Mehrwertsteuer-Einnahmen der Mitgliedstaaten, wie es in der Bundesrepublik Deutschland de facto der Fall ist, ist nicht zwingend. Die Mehrwertsteuer-Eigenmittel besitzen insofern den Charakter von Finanzbeiträgen.

\section{cc) Die Einnahmenkompetenzen bei den Bruttosozialprodukt-Eigenmitteln}

Zwischen den Bruttosozialprodukt-Eigenmitteln und einer nationalen Einnahmenart lassen sich keine Verbindungen feststellen, so daß kein spezieller Einnahmenverbund gegeben ist. ${ }^{17}$ Dennoch liegt nicht wie bei einem Trennsystem eine starke Einnahmenautonomie der Gemeinschaft vor, denn die Höhe der Bruttosozialprodukt-Eigenmittel wird durch die "Restfinanzierung" vorgegeben und ist insofern nicht frei variierbar. Da auch hier die Mitgliedstaaten und nicht die Bürger oder Unternehmen der Europäischen Gemeinschaften die Zahlungsverpflichteten sind, stellen die Bruttosozialprodukt-Eigenmittel ebenfalls nichts anderes als Finanzbeiträge dar. ${ }^{18}$ Mit der Einführung der Bruttosozialprodukt-Eigenmittel erfolgte keine Weiterentwicklung des Eigenmittelsystems im Sinne von Art. 201 EWGV-Vertrag. 19

\section{b) Die Schwierigkeiten bei der Deckung der Gemeinschaftsausgaben}

Wie im ersten Teil der Arbeit gezeigt wurde, ist eine ausreichende Einnahmenautonomie notwendig, um die zugewiesenen Aufgaben erfüllen zu können. Die Einnahmen müssen für die Aufgabenfinanzierung ausreichen und zwar auf längere Sicht. Das bedeutet zunächst, daß die notwendigen Ausgaben geleistet werden können. Bei dieser Forderung wird allerdings vorausgesetzt, daß die Probleme der Ausgabenverteilung sinnvoll gelöst worden sind. Ist dies nicht

16 Messal =EG-Finanzierung $=137$.

$17 \mathrm{Vgl}$. Messal =EG-Finanzierung $=138$.

$18 \mathrm{Vgl}$. Caesar $=$ Neue Steuern für die $E G=59 \mathrm{f}$.

19 Vgl. Messal =EG-Finanzierung $=138$. 
der Fall, wird auch das Ziel, die Ausgaben durch die Einnahmen zu decken, problematisch.

Die Ausgaben der Gemeinschaft konnten in den vergangenen Jahren nicht immer gedeckt werden. Im Eigenmittelbeschluß von 1970 war vorgesehen, daß die Mehrwertsteuer-Eigenmittel der Restfinanzierung dienen sollen. Dabei waren sie auf einen maximalen Satz von $1 \%$ der Bemessungsgrundlage für jedes Mitgliedsland begrenzt. Die Ausgaben bestimmten also die Höhe der Mehrwertsteuer-Eigenmittel. Da die Ausgaben jedoch - vor allem im Agrarsektor stark anstiegen, wurde schließlich der Plafonds von 1\% erreicht. Im Jahr 1984 wurde die Finanzkrise der Europäischen Gemeinschaft akut; es stellte sich während des Haushaltsjahres heraus, daß der geplante Budgetausgleich nicht erreicht werden konnte. Die Kommission schlug vor, daß die Mitgliedstaaten der Gemeinschaft rückzahlbare Vorschüsse auf die Eigenmittel des folgenden Haushaltsjahres einräumen sollten. Die Rückzahlungen sollten vom 30.6.1986 an aus dem nach einer Erhöhung der regulären Eigenmittel geschaffenen Einnahmenspielraum erfolgen. Auch 1985 mußten wieder Vorschüsse geleistet werden. ${ }^{20}$ Diese Vorschüsse in Form rückzahlbarer Darlehen bedeuten praktisch, daß die Gemeinschaft Schulden (bei den Mitgliedstaaten) zur Finanzierung ihres Haushaltes gemacht hat. ${ }^{21}$

Um die Finanzierungskrise zu entschärfen, wurde 1985 der MehrwertsteuerEigenmittelplafonds auf 1,4\% angehoben. Dies bot jedoch auf Dauer auch keine Lösung. Im Jahre 1988 wurde das Finanzierungssystem erneut geändert und die neue Eigenmittelart der Bruttosozialprodukt-Eigenmittel eingeführt. $\mathrm{Ob}$ die Finanzkrise der Europäischen Gemeinschaften damit dauerhaft behoben werden kann, bleibt fraglich, solange nicht die Probleme der Ausgabenseite, vor allem der Anstieg der Agrarausgaben, beseitigt werden. Dabei sei noch einmal darauf hingewiesen, daß die Ausgaben für den Agrarfonds mit einer gewissen Eigendynamik wachsen, die kaum über das Ausgabenbewilligungsverfahren zu kontrollieren ist, sondern Eingriffe in zugrunde liegende Rechtsakte erfordert. Erste Schritte in Richtung einer Eindämmung der Agrarausgaben wurden mit der Einführung der Agrarleitlinie und der sogenannten

20 Während 1984 als Vorschuß 1.242,4 Mio. ECU in Anspruch genommen wurden, umfaßte 1985 die Deckungslücke 1.674,6 Mio. ECU. Vgl. Münch =Verbindlichkeiten im EG-Haushalt $=97$.

21 Vgl. Timmann $=$ Finanzreform $=26$. 
Stabilisatoren unternommen. Ob diese Maßnahmen allerdings wirksam sind, ist fraglich. 22

Solange die Agrarausgaben die Ausgabenseite des Haushaltes der Europäischen Gemeinschaften dominieren, wird die Diskussion um (neue) Einnahmen verengt auf die Wahl, Mittel für eine Beibehaltung der Agrarpolitik zu beschaffen oder neue Mittel zu verweigern, um auf diese Weise eine Reform der Agrarpolitik zu erzwingen. ${ }^{23}$

\section{c) Fehlende Rückkopplungseffekte zwischen der Belastung der Bürger mit Abgaben und den Ausgaben der Europäischen Gemeinschaft}

Die voranstehenden Überlegungen haben deutlich gemacht, daß es bei der Einnahmenautonomie nicht nur um die Deckung der anfallenden Ausgaben geht, sondern auch um den Zusammenhang zwischen Einnahmen und Ausgaben. Eine sparsame Haushaltsführung läßt sich nur gewährleisten, wenn es Rückkopplungseffekte zwischen der Belastung der Bürger mit Abgaben und den Ausgaben gibt. Entsprechende Kontrollmechanismen kommen bei den Europäischen Gemeinschaften jedoch aus zwei Gründen nicht zum Tragen: erstens sind die wichtigsten Abgaben der Europäischen Gemeinschaften nicht merklich, zweitens lassen die Mängel im demokratischen System der Gemeinschaft Rückmeldungen von den Bürgern kaum zu.

\section{aa) Die fehlende Merklichkeit}

In der Literatur ist $\mathrm{z}$. $\mathrm{T}$. bezweifelt worden, daß die Merklichkeit eine sinnvolle Forderung an das Einnahmensystem der Europäischen Gemeinschaft sei, da ein nur gering ausgeprägtes 'Solidarbewußtsein' der Bürger vermutet wurde, von dem man negative Einflüsse auf den weiteren Integrationsprozeß befürchtete. ${ }^{24}$ Wenn jedoch keine "Verantwortungseinheit" zwischen der Belastung der Bürger mit Abgaben und den Ausgaben vorhanden ist, wird auch keine sparsame Haushaltsführung möglich sein. Gerade in den Europäischen Gemeinschaften wäre es sehr hilfreich gewesen, wenn eine merkliche Abgabe als "proper check on public expenditure" gewirkt hätte.

22 Vgl. dazu die Ausführungen unter D. III. 2.

23 Vgl. zu diesem Problem auch Bieber $=$ Neue Finanzierungsmittel $=39 \mathrm{f}$.

24 So $z$. B. Henke =Finanzierung der $\mathrm{EG}=30$. 
Bei der Charakterisierung der Eigenmittel hat die Kommission ausdrücklich darauf hingewiesen, daß Eigenmittel "unmittelbar von Einzelpersonen oder Unternehmen in der Gemeinschaft aufzubringen sind"25. Dieser direkte $\mathrm{Zu}$ griff existiert bisher nur bei den "originären" Eigenmitteln und den Umlagen der Europäischen Gemeinschaft für Kohle und Stahl. Bei den MehrwertsteuerEigenmitteln und den Bruttosozialprodukt-Eigenmitteln handelt es sich - wie oben gezeigt wurde - dagegen unter diesem Aspekt immer noch um Finanzbeiträge.

Die Verwirklichung einer direkt am Bürger ansetzenden, merklichen Gemeinschaftsteuer wurde in neuerer Zeit auch von der Kommission gefordert. ${ }^{26} \mathrm{Sie}$ stößt allerdings noch auf große Schwierigkeiten. Bei früheren Vorschlägen der Kommission, eine Steuer auf den Verbrauch von Tabak, Alkohol, Benzin oder Energie zu erheben ${ }^{27}$, tritt das Problem auf, daß diese Steuern einzeln nicht in der Lage wären, den Finanzbedarf zu decken. Das wichtigste Argument gegen diese Art von Gemeinschaftsteuern ist allerdings, daß in den einzelnen Mitgliedstaaten unterschiedliche Konsumgewohnheiten herrschen und damit einzelne nationale Konsumgewohnheiten diskriminiert würden. Ist der Konsum bestimmter Güter, etwa von Kaffee, Wein oder Bier in Ländern mit einem geringen Bruttosozialprodukt besonders hoch, ergeben sich darüber hinaus auch Verteilungsprobleme. ${ }^{28}$

Der Beitragsgerechtigkeit könnte durch eine Einkommensbesteuerung Rechnung getragen werden. Auch aufgrund fiskalischer Überlegungungen wäre eine Finanzierung der Europäischen Gemeinschaften durch die Belastung der personalen Einkommen vorteilhaft, da langfristig mit ausreichenden Erträgen gerechnet werden kann. ${ }^{29}$ Die Einführung einer entsprechenden Steuer dürfte bei der gegenwärtig herrschenden und in absehbarer Zukunft nicht geringer werdenden Heterogenität der Strukturen der Einkommensbesteuerung in den Mitgliedstaaten jedoch auf große Widerstände stoßen. ${ }^{30}$ Die großen Unterschiede in der Einkommensbesteuerung zwischen den Mitgliedstaaten sind nicht zuletzt

25 Vgl. Kommission der EG = Finanzierung des Gemeinschaftshaushalts $=17$.

26 Vgl. o. V. = Europa-Steuer $=15$.

27 Vgl. Kommission der EG $=$ Finanzierung des Gemeinschaftshaushalts $=22 \mathrm{ff}$.

28 Vgl. Henke =Finanzierung der $\mathrm{EG}=59$.

29 Vgl. Caesar $=$ Neue Steuern für die EG $=94$.

$30 \mathrm{Vgl}$. Biehl, Winter $=$ EG-Finanzierung $=82 \mathrm{f}$. 
ein Spiegel stark differierender allokationspolitischer und distributionspolitischer Vorstellungen im nationalen Bereich. ${ }^{31}$ " $\mathrm{Da}$ im übrigen zwischen der Einkommensbesteuerung und dem Bereich der Sozialabgaben gewisse Substitutionsbeziehungen unverkennbar sind, wären überdies vermutlich parallele Harmonisierungsschritte auch bei den Sozialabgaben erforderlich. "32 Darüber hinaus müßten eine ganze Reihe technischer Schwierigkeiten gelöst werden. Eine gemeinschaftliche Besteuerung der Einkommen wird daher wohl erst für die Endphase der europäischen Integration in Frage kommen können. ${ }^{33}$

Ein Vorschlag, der auf Biehl und Winter ${ }^{34}$ zurückgeht, versucht, an der Besteuerung des Einkommens festzuhalten und die Harmonisierungsprobleme zu umgehen. Er sieht eine zweistufige Zuschlagsbesteuerung auf die nationalen Einkommen- und Körperschaftsteuern vor. Nach diesem Konzept wird in der ersten Stufe festgelegt, welcher Betrag insgesamt von einem Mitgliedstaat zur Finanzierung der Gemeinschaft aufgebracht werden soll, wobei die nationale Belastung progressiv sein soll. ${ }^{35}$ Die Kommission schlägt die anzuwendenden Schlüssel zur Ermittlung der nationalen Beiträge vor; Rat und Parlament beschließen sie. Danach wird in einer zweiten Stufe die Belastung innerhalb der Mitgliedstaaten mittels eines Zuschlags zur individuellen Einkommen- und Körperschaftsteuerschuld umgelegt, d. h. der Betrag der nationalen Belastung wird umgerechnet in einen nationalen Zuschlagsteuersatz, der die individuelle Steuerzahllast der einzelnen Bürger angibt. Die Entscheidung über die Höhe des nationalen Zuschlagsatzes kann den Haushaltsorganen der Gemeinschaften übertragen werden; dann müßten die Politiker in diesen Organen die Verantwortung für die Besteuerung gegenüber den Bürgern übernehmen. Man könnte jedoch auch diese zweite Entscheidung den nationalen Parlamenten überlassen und der Gemeinschaft nur eine Rahmenkompetenz zuteilen. Dies würde jedoch eine Aufteilung der Verantwortung für die Steuerbeschlüsse bedeuten. $\mathbf{3 6}$

31 Vgl. Caesar $=$ Neue Steuern für die $\mathrm{EG}=95$.

32 Caesar $=$ Neue Steuern für die $\mathrm{EG}=95$.

33 So auch Caesar $=$ Neue Steuern für die $E G=95 f$.

34 Vgl. Biehl, Winter $=$ EG-Finanzierung $=86 \mathrm{ff}$.

35 Auf die schwierige Frage, wie ein Progressionsgrad festgelegt werden soll, gehen Biehl und Winter nicht ein.

36 Vgl. Biehl, Winter $=$ EG-Finanzierung $=87$. 
Dieses Zuschlagsverfahren vermeidet die Notwendigkeit einer einheitlichen Einkommen- und Körperschaftsteuer, erschließt den Europäischen Gemeinschaften aber trotzdem ein flexibles und verteilungsgerechtes Einnahmeninstrument. Die finanzielle Belastung durch die Europäischen Gemeinschaften wäre für den Steuerpflichtigen fühlbar und damit transparenter. ${ }^{37}$

Ein solches Zuschlagssystem wäre auch im Hinblick auf die Verwaltungshoheit sinnvoll. Solange die Europäischen Gemeinschaften bei der Durchsetzung ihres Ertragsanspruchs auf die Mitwirkung der nationalen Behörden angewiesen sind, ist es für sie günstiger, wenn die nationalen Regierungen ein Interesse daran haben, die gemeinsame Steuerquelle zu pflegen und den Steueranspruch voll durchzusetzen. Bei einem Trennsystem könnte das Interesse der nationalen Regierungen, ihre Wirtschaftssubjekte zugunsten der Europäischen Gemeinschaften steuerlich zu belasten, gering sein. ${ }^{38}$

Gegen den Vorschlag Biehls und Winters wurde eingewandt, daß für Bürger mit gleichen Einkommen unterschiedliche Belastungen je nach Wohnsitzland entstünden und dies zu Diskriminierungen unter den Bürgern der Gemeinschaft führe. ${ }^{39}$ Dem kann jedoch entgegengehalten werden, daß die Unterschiede der Einkommensteuersysteme der Mitgliedstaaten den unterschiedlichen Präferenzen in diesen Staaten entsprechen. Der Vorschlag, den Zuschlagsatz der Gemeinschaft auf die nationale Einkommensteuerschuld anzurechnen, um dem Aspekt der Ungleichbehandlung Rechnung zu tragen, hilft dagegen nicht weiter ${ }^{\mathbf{4 0}}$, denn dadurch bekommt die Abgabe wiederum de facto den Charakter eines Finanzbeitrags. Da hiermit außerdem große Auswirkungen auf das nationale Steueraufkommen verbunden wären, ist eine Zustimmung des Rates zu solchen Überlegungen nicht sehr wahrscheinlich.

\section{bb) Sicherung der Rückkopplungseffekte durch eine verstärkte Haushalts- autonomie des Europäischen Parlaments}

Die politische Rückkopplung ist nicht allein durch die Einführung einer wirklichen Gemeinschaftsteuer gesichert. Auch wenn die Bürger über ihre Belastun-

37 Vgl. Fischer $=$ EG-Zuschlag $=138$ und Ungar $=$ EG-Finanzverfassung $=129$.

$38 \mathrm{Vgl}$. Peffekoven $=$ Eigene Einnahmen $=323 \mathrm{f}$.

39 Vgl. dazu Stoll = Alternativen der EG-Finanzierung $=144$.

$40 \mathrm{Vgl}$. Ungar =EG-Finanzverfassung $=129$. 


\section{Einnahmenautonomie}

gen Bescheid wüßten, wäre es für sie schwieriger als auf nationaler Ebene, ihre Interessen zu wahren, da sie (durch Wahlen) nur auf das Europäische Parlament, nicht aber auf den Rat Einfluß nehmen können. Im Augenblick kann das Europäische Parlament den Haushalt nur im Rahmen der nicht-obligatorischen Ausgaben - also nur sehr begrenzt - mitgestalten. Das Parlament hat keine Steuergesetzgebungshoheit - eine Kompetenz, die zu den Kernelementen parlamentarischer Arbeit der nationalen Parlamente gehört. Damit besteht der für demokratische Systeme wesentliche Entscheidungszusammenhang zwischen Besteuerungskompetenz und Ausgabenkompetenz für die Europäischen Gemeinschaften nicht. Hier zeigen sich die demokratischen Defizite der Europäischen Gemeinschaften in besonderer Weise.

Für die Finanzautonomie der Europäischen Gemeinschaften ist es daher nur eine notwendige, aber keine hinreichende Bedingung, eine direkt am Bürger ansetzende Gemeinschaftsteuer einzuführen. Um die Rückkopplungseffekte zu sichern, müßte als weitere Bedingung dem Europäischen Parlament diese Einnahmenautonomie zugesprochen werden. ${ }^{41}$ Die politische Forderung "no taxation without representation" hat insofern einen ökonomischen Hintergrund.

41 So auch Caesar $=$ Neue Steuern für die $E G=73$. 


\section{Die Diskussion um einen angemessenen Finanzierungsanteil}

Die Tatsache, daß die ertragreichsten Einnahmen der Europäischen Gemeinschaften - Mehrwertsteuer-Eigenmittel und Bruttosozialprodukt-Eigenmittel de facto den Charakter von Finanzzuweisungen haben, bildet auch den Hintergrund für die Diskussion um den "gerechten Finanzierungsbeitrag" der einzelnen Mitgliedstaaten. Für diese Diskussion ist grundlegend, daß die einzelnen Mitgliedstaaten die Schuldner der Abgaben sind und die innere Aufbringung im Ermessen der Mitgliedstaaten steht. ${ }^{1}$ Insofern unterscheidet sie sich auch nicht von den Überlegungen zu einer "gerechten" Lastenverteilung anderer internationaler Organisationen.

\section{Die Forderung nach "juste retour"}

In der Diskussion um eine "gerechte" Lastenverteilung in den Europäischen Gemeinschaften spiegeln sich sowohl der Äquivalenzansatz als auch der Ansatz der Lastenverteilung nach der Wirtschaftskraft wider. Der Forderung nach "juste retour", die Großbritannien bei den Verhandlungen vertrat und die im Rahmen der Korrektur für Großbritannien auch durchgesetzt wurde, liegen äquivalenztheoretische Vorstellungen zugrunde. Allerdings läßt sich diese Forderung erst gar nicht auf eine exakte Erfassung der Nutzen ein, sondern vergleicht lediglich die Finanzierungsanteile der Mitgliedstaaten mit den Rückflüssen aus dem Haushalt an die einzelnen Mitgliedstaaten. Bei dieser vereinfachten Äquivalenzvorstellung sind die den Mitgliedstaaten zurechenbaren Ausgaben eine Art Indikator für den Nutzen. Allerdings ist dieser Indikator höchst zweifelhaft; denn durch eine Reduktion der Betrachtung auf die Budgetaktivitäten wird nur ein geringer Teil der ökonomischen Integrationseffekte erfaßt. ${ }^{2}$ Insbesondere bei einer wirtschaftlichen Integration sind die Kosten und Nutzen einer Mitgliedschaft zumeist nur in geringem Maße in den Ausgaben und Einnahmen begründet. ${ }^{3}$ Der "Nachteil" einer Nettozahlungsposition kann

1 Vgl. dazu auch Caesar = Neue Steuern für die $E G=58$.

2 Vgl. Hackenbroch $=$ Supranationaler Finanzausgleich $=114$. Zur Kritik an dieser Praxis vgl. auch Nittka $=$ Finanzierungssystem $=231 \mathrm{f}$.

$3 \quad$ Vgl. Nittka $=$ Finanzierungssystem $=231$. 
durch nicht-budgetäre Aktivitäten vergrößert, kompensiert oder überkompensiert werden. ${ }^{4}$

Trotz dieser Einwände führte die britische Forderung nach "juste retour" zu einem Ausgleich für Großbritannien. Dabei gingen allerdings auch Elemente einer Lastenverteilung entsprechend der Wirtschaftskraft in die Überlegungen mit ein, denn die durch den Vergleich der Finanzierungsbeiträge mit den Rückflüssen ermittelten Nettopositionen der Mitgliedstaaten wurden mit Blick auf die wirtschaftliche Leistungsfähigkeit beurteilt. Schon 1974 hatte Großbritannien darauf hingewiesen, daß zwischen seinen Zahlungen und Rückflüssen angesichts seines relativen Wohlstandes ein Mißverhältnis bestehe, und Korrekturen gefordert. Als Folge wurde 1976 ein allgemeiner Finanzmechanismus beschlossen, bei dem unter bestimmten Bedingungen der Finanzierungsanteil eines Mitgliedstaates reduziert werden konnte. Voraussetzungen dafür waren u. a. eine Nettozahlerposition und die Unterschreitung vorgegebener Bruttosozialprodukt- oder Bruttoinlandsprodukt-Relationen. Dieser Finanzmechanismus kam allerdings nie zur Anwendung, da kein Mitgliedstaat - auch nicht Großbritannien - alle Ausgleichsvoraussetzungen gleichzeitig erfültte. ${ }^{5}$ In den siebziger Jahren war das Ausgleichsproblem jedoch nicht so drängend, da Großbritannien aufgrund der Beitrittsbestimmungen ein Teil seiner Beiträge erstattet wurde.

In den Jahren 1980 bis 1983 erhielt Großbritannien Ad-hoc-Ausgleichsbeträge. Im Eigenmittelbeschluß von 1985 wurde ein allgemeiner Ausgleichsgrundsatz beschlossen, der dann durch den Korrekturmechanismus für Großbritannien konkretisiert wurde. Darin heißt es: "Jeder Mitgliedstaat, der gemessen an seinem relativen Wohlstand eine zu große Haushaltslast trägt, kann zu gegebener Zeit in den Genuß einer Korrekturmaßnahme gelangen. "6 Eine eindeutige Konkretisierung, was "relativer Wohlstand" und "zu große Haushaltslast" heißt, wurde allerdings nie getroffen.

In den Jahren 1981 bis 1984 waren nur Deutschland und Großbritannien Nettozahler, während alle anderen Mitgliedstaaten ${ }^{7}$ Nettoempfänger waren (siehe Tabelle 26). Zieht man das Bruttoinlandsprodukt-pro-Kopf zur Beurteilung der

$4 \quad$ Vgl. Nittka $=$ Finanzierungssystem $=232$.

$5 \quad \mathrm{Vgl}$. Messal $=$ EG-Finanzierung $=25 \mathrm{f}$.

6 Artikel 3 des Beschlusses des Rates 85/257 vom 7.5.1985.

7 Mit Ausnahme Frankreichs 1984. 
Tabelle 26: Nettopositionen und Bruttoinlandsprodukt-pro-Kopf1) in \% des Gemeinschaftsdurchschnitts der Mitgliedstaaten in den Jahren 1981 bis 1984

\begin{tabular}{|c|c|c|c|c|c|c|c|c|}
\hline \multirow{2}{*}{$\begin{array}{l}\text { Mitglied- } \\
\text { staat }\end{array}$} & \multicolumn{4}{|c|}{ Nettopositionen (in Mio. ECU) } & \multirow{2}{*}{\multicolumn{3}{|c|}{$\begin{array}{l}\text { BIP-pro-Kopf (in \% des } \\
\text { Gemeinschaftsdurchschnitts) }\end{array}$}} & \multirow[b]{2}{*}{1984} \\
\hline & 1981 & 1982 & 1983 & 1984 & & & & \\
\hline \multicolumn{9}{|c|}{ Nettoempfänger } \\
\hline $\begin{array}{l}\text { B } \\
\text { DK } \\
\text { GRL } \\
\text { F }\end{array}$ & $\begin{array}{l}+320 \\
+\quad 280 \\
+\quad 160 \\
+540\end{array}$ & $\begin{array}{r}+\quad 310 \\
+\quad 280 \\
+\quad 690 \\
0\end{array}$ & $\begin{array}{l}+210 \\
+\quad 330 \\
+\quad 940 \\
+\quad 10\end{array}$ & $\begin{array}{r}+330 \\
+500 \\
+\quad 990 \\
-\end{array}$ & $\begin{array}{r}97,8 \\
103,2 \\
55,1 \\
107,1\end{array}$ & $\begin{array}{r}98,9 \\
105,8 \\
54,7 \\
108,8\end{array}$ & $\begin{array}{r}97,5 \\
107,1 \\
53,8 \\
107,7\end{array}$ & $\begin{array}{r}97,5 \\
108,4 \\
53,8 \\
106,3\end{array}$ \\
\hline $\begin{array}{l}\text { IRL } \\
\text { I } \\
\text { LUXX } \\
\text { NL }\end{array}$ & $\begin{array}{l}+590 \\
+\quad 780 \\
+\quad 270 \\
+\quad 220\end{array}$ & $\begin{array}{r}+740 \\
+1.520 \\
+\quad 250 \\
+350\end{array}$ & $\begin{array}{r}+770 \\
+1.320 \\
+270 \\
+370\end{array}$ & $\begin{array}{r}+920 \\
+1.710 \\
+\quad 280 \\
+\quad 510\end{array}$ & $\begin{array}{r}62,9 \\
97,9 \\
111,0 \\
104,3\end{array}$ & $\begin{array}{r}63,3 \\
97,4 \\
112,1 \\
101,8\end{array}$ & $\begin{array}{r}61,6 \\
96,3 \\
113,9 \\
101,4\end{array}$ & $\begin{array}{r}61,7 \\
97,1 \\
118,4 \\
102,2\end{array}$ \\
\hline \multicolumn{9}{|c|}{ Nettozahler } \\
\hline $\begin{array}{l}\mathrm{D} \\
\mathrm{UK} \\
\mathrm{F}\end{array}$ & $\begin{array}{r}-1.770 \\
-1.380\end{array}$ & $\begin{array}{r}-2.320 \\
-1.820 \\
0\end{array}$ & $\begin{array}{r}-2.300 \\
-1.910\end{array}$ & $\begin{array}{r}-2.950 \\
-1.950 \\
330\end{array}$ & $\begin{array}{r}108,6 \\
94,8 \\
107,1\end{array}$ & $\begin{array}{r}107,2 \\
95,5 \\
108,8\end{array}$ & $\begin{array}{r}108,0 \\
97,4 \\
107,7\end{array}$ & $\begin{array}{r}108,7 \\
96,7 \\
106,3\end{array}$ \\
\hline
\end{tabular}

Quelle: Messal =EG-Finanzierung $=22$ und 24 . 
Tabelle 27: Finanzierungsanteile und Anteile am Bruttoinlandsprodukt (BIP) der Mitgliedstaaten 1981 bis 1984

\begin{tabular}{|lrrrrrrrr|}
\hline \multicolumn{3}{c}{ Finanzierungsanteile } & \multicolumn{5}{c|}{ BIP-Anteile } \\
\hline & & & & & & & \\
& 1981 & 1982 & 1983 & 1984 & 1981 & 1982 & 1983 & 1984 \\
& & & & & & & & \\
DK & 5,5 & 5,4 & 5,3 & 5,0 & 3,79 & 3,49 & 3,39 & 3,38 \\
D & 2,0 & 1,9 & 2,1 & 2,1 & 2,25 & 2,29 & 2,35 & 2,39 \\
GRL & 28,0 & 26,9 & 28,1 & 28,4 & 26,84 & 27,04 & 27,56 & 27,27 \\
F & 1,4 & 1,8 & 1,6 & 1,4 & 1,46 & 1,58 & 1,47 & 1,50 \\
IRL & 19,4 & 20,0 & 19,6 & 19,3 & 22,94 & 22,67 & 22,11 & 22,08 \\
I & 0,9 & 1,0 & 1,2 & 1,2 & 0,72 & 0,78 & 0,77 & 0,78 \\
LUX & 14,0 & 11,8 & 13,0 & 13,8 & 16,22 & 16,56 & 17,49 & 18,13 \\
NL & 0,2 & 0,1 & 0,2 & 0,2 & 0,15 & 0,14 & 0,14 & 0,15 \\
UK & 7,1 & 6,9 & 6,8 & 6,8 & 5,5 & 5,67 & 5,61 & 5,51 \\
& 21,5 & 24,2 & 22,1 & 21,8 & 20,07 & 19,77 & 19,10 & 18,78 \\
\hline
\end{tabular}

Quelle: Messal $=$ EG-Finanzierung $=24$. 
Nettopositionen als Maßstab für den Wohlstand heran, so zeigt sich insofern ein Ungleichgewicht, als Großbritannien in den Jahren 1981 bis 1984 ein unter dem europäischen Durchschnitt liegendes Bruttoinlandsprodukt-pro-Kopf aufwies und dennoch Nettozahler war, während Staaten mit überdurchschnittlichem Bruttoinlandsprodukt-pro-Kopf, wie z. B. die Niederlande oder Dänemark, Nettoempfänger waren.

Auch bezogen auf die Anteile am Bruttoinlandsprodukt als Indikatoren für die Wirtschaftskraft ergab sich ein Ungleichgewicht (siehe Tabelle 27). Der Finanzierungsanteil Großbritanniens lag deutlich über seinem Anteil am gesamten Bruttoinlandsprodukt der Europäischen Gemeinschaft. Zwar war dies auch in anderen Mitgliedstaaten der Fall (z. B. in Belgien, in Irland und in den Niederlanden), doch waren diese Mitgliedstaaten eben Nettoempfänger. Die Korrektur für Großbritannien sollte eingeführt werden, weil seine Nettozahlerposition nicht seiner wirtschaftlichen Leistungsfähigkeit entsprach. Die Nettoposition der Bundesrepublik Deutschlands wurde dagegen aufgrund ihrer wirtschaftlichen Leistungsfähigkeit nie grundsätzlich in Frage gestellt. Sie wurde lediglich bei der Finanzierung des Ausgleichs für Großbritannien berücksichtigt; die Bundesrepublik Deutschland muß hier nur zwei Drittel ihres eigentlichen Anteils tragen.

Während bei der bisherigen Betrachtung der Finanzierungsanteile Zölle und Agrarabschöpfungen mitberücksichtigt wurden, klammerte man diese Eigenmittelarten bei der Konstruktion des Korrekturmechanismus aus. Diese Mittel haben nicht den Charakter von Finanzzuweisungen, vielmehr ist bei ihnen ein direkter Zugriff auf Unternehmen und Bürger gegeben, und sie sind als originäre Eigenmittel der Europäischen Gemeinschaften nicht mehr einzelnen Mitgliedstaaten zuzuordnen. ${ }^{8}$

Die Höhe der von einem Mitgliedstaat abzuführenden Zolleinnahmen wird vom Volumen der Drittlandsimporte, von dem darauf lastenden Zollniveau und durch die Zusammensetzung der Drittlandsimporte nach Einfuhrländern und Warengruppen bestimmt.

Während die - bezogen auf das Bruttoinlandsprodukt - hohen Zollabführungen Großbritanniens und Irlands (siehe Tabelle 28) in erster Linie aus der Handelsund Warenstruktur des Importes resultieren, sind die hohen Werte Belgiens

8 Vgl. dazu u. a. Messal $=$ EG-Finanzierung $=28$. 
Tabelle 28: Die Relation der Anteile der Agrarabschöpfungen und der Zölle der Mitgliedstaaten zum Anteil am Bruttoinlandsprodukt (BIP) der Gemeinschaft in den Jahren 1986 bis 1988

\begin{tabular}{|c|c|c|c|c|c|c|}
\hline \multirow[t]{2}{*}{$\begin{array}{l}\text { Mitglied- } \\
\text { staat }\end{array}$} & \multicolumn{3}{|c|}{$\begin{array}{l}\text { Anteil der Zölle } \\
\text { zu BIP-Anteil }\end{array}$} & \multicolumn{3}{|c|}{$\begin{array}{c}\text { Anteil der Agrarabschöp } \\
\text { fungen zu BIP-Anteil }\end{array}$} \\
\hline & 1986 & 1987 & 1988 & 1986 & 1987 & 1988 \\
\hline LUX & 0,50 & 0,57 & 0,46 & 0,14 & 0,07 & 0,07 \\
\hline $\mathrm{D}$ & 1,37 & 1,36 & 1,35 & 0,44 & 0,44 & 0,58 \\
\hline DK & 1,42 & 1,21 & 1,23 & 0,44 & 0,53 & 0,73 \\
\hline $\mathrm{F}$ & 0,74 & 0,72 & 0,71 & 0,48 & 0,35 & 0,39 \\
\hline UK & 1,25 & 1,21 & 1,26 & 1,33 & 1,36 & 0,96 \\
\hline I & 0,51 & 0,53 & 0,52 & 1,64 & 1,37 & 1,45 \\
\hline NL & 2,02 & 1,95 & 1,95 & 2,02 & 1,27 & 1,54 \\
\hline B & 1,95 & 1,91 & 1,92 & 3,63 & 5,65 & 3,09 \\
\hline E & 0,27 & 0,48 & 0,45 & 1,48 & 0,54 & 0,10 \\
\hline IRL & 2,00 & 1,86 & 1,86 & 0,77 & 0,51 & 0,29 \\
\hline GRL & 0,62 & 0,61 & 0,71 & 1,15 & 0,69 & 0,72 \\
\hline $\mathbf{P}$ & 0,38 & 0,66 & 0,64 & 0,99 & 1,59 & 1,70 \\
\hline
\end{tabular}

Quelle: Europäischer Rechnungshof =Jahresbericht $1988=229$, Statistisches Bundesamt $=$ Ausland $1989=100$ und eigene Berechnungen. 
und der Niederlande aus ihren weit überdurchschnittlichen Importquoten zu erklären. ${ }^{9}$

Hier zeigt sich, daß bei den Zöllen nicht an den reinen Zahlungsströmen als Maßstab für die finanzielle Belastung der Mitgliedstaaten angeknüpft werden kann, denn Zölle werden denjenigen Mitgliedstaaten als Zahlungen angerechnet, in denen diese Einnahmen anfallen. Wenn nun die Importländer nicht gleichzeitig die Bestimmungsländer der Waren innerhalb der Europäischen Gemeinschaften sind, werden ihnen Abführungen anerkannt, die ihre Bürger nicht belasten, d. h. das Aufkommen der Abgaben und ihre Traglast fallen auseinander. ${ }^{10}$

Für ein Auseinanderfallen von Importland und Bestimmungsland sind vor allem geographische und transporttechnische Gegebenheiten maßgeblich. Da insbesondere den großen Häfen Rotterdam und Antwerpen eine erhebliche Bedeutung in diesem Zusammenhang zukommt, hat sich für die Charakterisierung des Problems auch der Begriff "Rotterdam-Antwerpen-Effekt"11 herausgebildet. Ein vergleichbarer Effekt ergibt sich auch bei den Agrarabschöpfungen, die den europäischen Agrarmarkt nach außen abschotten. ${ }^{12}$ Es ist daher sinnvoll, Zölle und Agrarabschöpfungen nicht mit in die Frage nach einer gerechten Lastenverteilung einzubeziehen.

Bei der Konstruktion des Korrekturmechanismus wird also nur auf die Mehrwertsteuer-Eigenmittel und die Bruttosozialprodukt-Eigenmittel zurückgegriffen, eben die Einnahmen, die Finanzbeitragscharakter haben. Nur dieser Teil der Einnahmen wird mit den anteiligen Rückflüssen verglichen. Nach 1984 hat die Kommission keine Angaben zu den Nettopositionen mehr gemacht, da sie der rein finanziellen Betrachtungsweise der Vorteile und Nachteile einer Mitgliedschaft nicht Vorschub leisten wollte. ${ }^{13}$ Der Europäische Rechnungshof weist für den größten Teil der Ausgaben (ca. 87\%) zwar die Mittelzuflüsse in die einzelnen Mitgliedstaaten aus ${ }^{\mathbf{1 4}}$, doch exakte Nettopositionen lassen sich damit nicht errechnen. Zu berücksichtigen ist allerdings, daß nicht alle Aus-

9 Vgl. Ott $=$ Verteilungswirkungen $=94 \mathrm{ff}$.

$10 \mathrm{Vgl}$. Ott $=$ Verteilungswirkungen $=100$.

$11 \mathrm{Ott}=$ Verteilungswirkungen $=101$.

12 Vgl. dazu Ott $=$ Verteilungswirkungen $=111$.

13 Vgl. dazu Messal =EG-Finanzierung $=51$.

14 Vgl. Europäischer Rechnungshof =Jahresbericht 1989= Tabellenteil 76. 


\section{Die Diskussion um einen angemessenen Finanzierungsanteil}

gaben in die Mitgliedstaaten zurückfließen, wie z. B. die Ausgaben für die Zusammenarbeit mit Drittländern und Entwicklungsländern. Die Angaben des Rechnungshofes lassen insofern Tendenzaussagen über die Rückflüsse zu.

Bei den Verhandlungen zur Reform des Finanzsystems im Jahre 1988 wurde gegen die Beibehaltung der Korrektur zugunsten Großbritanniens in der bestehenden Form eingewandt, daß Großbritannien nach dem Beitritt Spaniens und Portugals "relativ reicher" geworden sei; zwar gäbe es immer noch eine ungleiche Verteilung der Finanztransfersalden, aber Großbritannien liege jetzt wohlstandsmäßig über dem Durchschnitt der Gemeinschaft (siehe Tabelle 29); im übrigen werde Großbritannien durch die Einbeziehung des Bruttosozialprodukts in die Eigenmittel-Bemessungsgrundlagen bereits finanziell entlastet, von daher müsse es eine substantielle Verringerung seines Ausgleichsanspruchs hinnehmen. ${ }^{15}$ Diesen Einwänden wurde jedoch nicht Rechnung getragen. Großbritannien setzte durch, daß es im neuen System genauso gestellt wurde wie im alten. Die komplizierte Konstruktion des Ausgleichs seit 1988 stellt eine möglichst exakte Umsetzung dieses Grundsatzes dar. ${ }^{16}$

Tabelle 29: Bruttoinlandsprodukt-pro-Kopf* in den Jahren 1986 bis 1988 in \% des Gemeinschaftsdurchschnitts

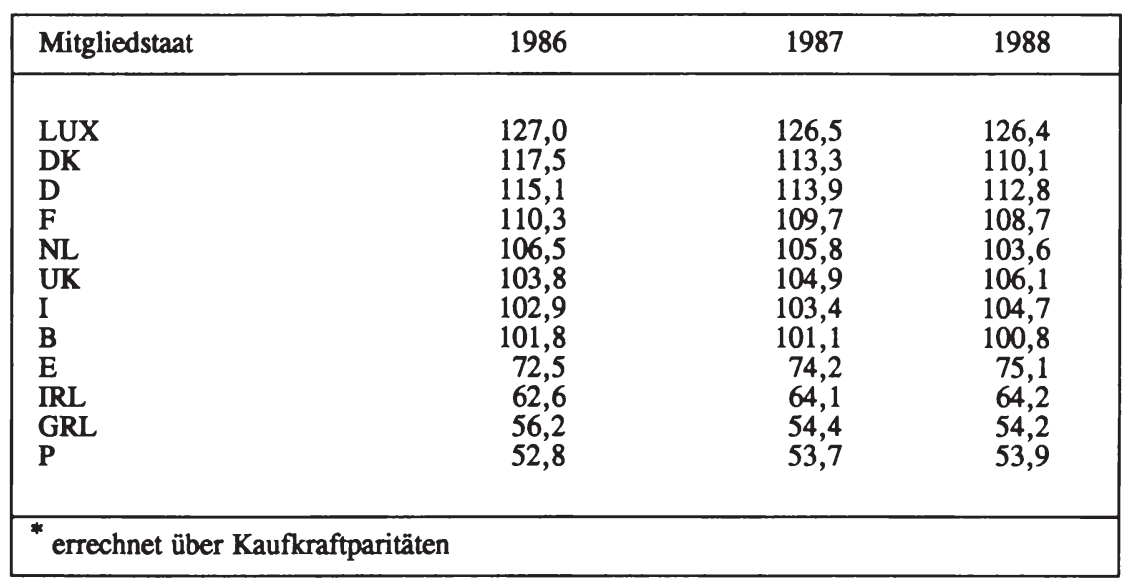

Quelle: Statistisches Bundesamt $=$ Ausland $1989=100$ und 102 und eigene Berechnungen.

15 Vgl. Messal $=$ EG-Finanzierung $=51$.

16 Vgl. Messal $=$ EG-Finanzierung $=55$. 
Tabelle 30: Die Auswirkungen des Korrekturmechanismus im Haushalt 1991

\begin{tabular}{|c|c|c|c|c|c|c|}
\hline $\begin{array}{l}\text { Mit- } \\
\text { glied- } \\
\text { staat }\end{array}$ & $\begin{array}{r}\text { MWSt-und BSP- } \\
\text { Anteile vor } \\
\text { der Korrektur }\end{array}$ & $\begin{array}{r}\text { MWSt-und BSP- } \\
\text { Anteile nach } \\
\text { der Korrektur }\end{array}$ & $\begin{array}{r}\text { Finanzierungs- } \\
\text { anteile vor } \\
\text { der Korrektur }\end{array}$ & $\begin{array}{r}\text { Finanzierungs- } \\
\text { anteile nach } \\
\text { der Korrektur }\end{array}$ & BSP-Anteile & $\begin{array}{l}\text { Anteile an } \\
\text { den anrechen- } \\
\text { baren Rück- } \\
\text { flüssen } 1989\end{array}$ \\
\hline B & 2,9 & 3,3 & 3,89 & 4,19 & 3,1 & 2,0 \\
\hline DK & 1,8 & 2,0 & 1,78 & 1,95 & 2,0 & 3,1 \\
\hline D & 26,4 & 28,2 & 26,91 & 28,23 & 26,7 & 13,4 \\
\hline E & 8,4 & 9,4 & 7,46 & 8,15 & 8,0 & 10,4 \\
\hline F & 20,0 & 22,2 & 18,45 & 20,09 & 19,0 & 16,7 \\
\hline GRL & 1,3 & 1,5 & 1,34 & 1,45 & 1,2 & 7,5 \\
\hline IRL & 0,7 & 0,7 & 0,84 & 0,89 & 0,6 & 5,0 \\
\hline I & 15,2 & 17,5 & 14,27 & 15,81 & 17,9 & 18,1 \\
\hline LUX & 0,2 & 0,2 & 0,16 & 0,18 & 0,2 & 0,0 \\
\hline NL & 4,6 & 5,1 & 5,67 & 6,06 & 4,5 & 11,2 \\
\hline $\mathbf{P}$ & 1,1 & 1,2 & 1,25 & 1,33 & 1,0 & 2,8 \\
\hline UK & 17,3 & 8,7 & 17,99 & 11,71 & 15,8 & 9,4 \\
\hline
\end{tabular}

Quelle: Europäisches Parlament $=$ Haushaltsplan $1991=$ und eigene Berechnungen. 
Tabelle 31: Die Mehrwertsteuer-Bemessungsgrundlage vor und nach der Kappung 1989

\begin{tabular}{|c|c|c|c|c|c|c|c|}
\hline \multirow[t]{2}{*}{$\begin{array}{l}\text { Mit- } \\
\text { glied- } \\
\text { staat }\end{array}$} & \multicolumn{2}{|c|}{$\begin{array}{r}\text { MWSt-Bemessungs- } \\
\text { grundlage vor } \\
\text { der Kappung }\end{array}$} & \multicolumn{2}{|c|}{$\begin{array}{r}\text { Bruttosozial- } \\
\text { produkt }\end{array}$} & \multirow{2}{*}{$\begin{array}{r}\text { Anteil der } \\
\text { MWSt-Bemes- } \\
\text { sungsgrundlage } \\
\text { am Brutto- } \\
\text { sozialprodukt }\end{array}$} & \multicolumn{2}{|c|}{$\begin{array}{r}\text { MWSt-Bemessungs- } \\
\text { grundlage nach } \\
\text { der Kappung }\end{array}$} \\
\hline & Mrd. ECU & $\%$ & Mrd. ECU & $\%$ & & Mrd. ECU & $\%$ \\
\hline B & 60,0 & 2,86 & 132,7 & 3,21 & 45,2 & 60,0 & 2,92 \\
\hline DK & 41,5 & 1,98 & 91,5 & 2,21 & 45,4 & 41,5 & 2,02 \\
\hline D & 523,6 & 24,95 & 1048,3 & 25,32 & 49,9 & 523,6 & 25,45 \\
\hline E & 152,8 & 7,28 & 294,8 & 7,12 & 51,8 & 152,8 & 7,40 \\
\hline F & 436,0 & 20,78 & 823,3 & 19,89 & 53,0 & 436,0 & 21,19 \\
\hline GRL & 23,0 & 1,10 & 52,3 & 1,26 & 44,0 & 23,0 & 1,12 \\
\hline IRL & 14,8 & 0,71 & 23,8 & 0,57 & 62,2 & 13,1 & 0,64 \\
\hline I & 306,0 & 14,58 & 743,7 & 17,96 & 41,1 & 306,0 & 4,87 \\
\hline LUX & 5,0 & 0,24 & 7,8 & 0,19 & 64,1 & 4,3 & 0,21 \\
\hline NL & 98,0 & 4,67 & 195,6 & 4,72 & 50,1 & 98,0 & 4,76 \\
\hline $\mathbf{P}$ & 25,0 & 1,19 & 36,7 & 0,89 & 68,1 & 20,2 & 0,98 \\
\hline UK & 412,7 & 19,67 & 689,6 & 16,66 & 59,8 & 379,3 & 18,43 \\
\hline Summe & 2098,4 & 100,00 & 4140,1 & 100,00 & - & 2057,8 & 100,00 \\
\hline
\end{tabular}

Quelle: Europäisches Parlament =Haushaltsplan 1989=, Angaben des Bundesministeriums der Finanzen und eigene Berechnungen. 
Die Auswirkungen des Korrekturmechanismus im derzeitigen Haushalt macht Tabelle 30 deutlich. Der Anteil Großbritanniens an den Mehrwertsteuer- und Bruttosozialprodukt-Eigenmitteln wird halbiert. Sein gesamter Finanzierungsanteil liegt nach der Korrektur deutlich unter seinem Anteil am Bruttosozialprodukt und auch unter dem Anteil an den zurechenbaren Rückflüssen. Der Finanzierungsanteil der Bundesrepublik Deutschland, der vor der Korrektur annähernd dem deutschen Anteil am Bruttosozialprodukt entsprach, wird durch die Korrektur deutlich über diesen Anteil gehoben und noch viel deutlicher über den Anteil an den zurechenbaren Rückflüssen.

Obwohl sich in den letzten Jahren die Wirtschaftskraft Großbritanniens nicht mehr gravierend von der deutschen Wirtschaftskraft unterschied, da das Bruttoinlandsprodukt-pro-Kopf beider Staaten deutlich über dem Gemeinschaftsdurchschnitt liegt (siehe Tabelle 29), und die Finanzierungsanteile beider Staaten vor der Korrektur ungefähr das Doppelte der zurechenbaren Rückflüsse betrug (siehe Tabelle 30), wurde nur Großbritannien durch die Korrektur entlastet. Die Bundesrepublik Deutschland muß die Entlastung mittragen, wenn auch nur mit zwei Dritteln ihres Anteils. Der Korrekturmechanismus trägt unter keinem Gesichtspunkt zu einer gerechteren Verteilung der Finanzierungslasten bei. Ihm liegt keine konsistente Konzeption zugrunde, er stellt lediglich eine Sonderregelung dar, die von Großbritannien politisch durchgesetzt wurde und die das Einnahmensystem der Europäischen Gemeinschaften komplizierter und undurchschaubarer macht. Er sollte abgeschafft werden.

\section{Die Kappung der Mehrwertsteuer-Bemessungsgrundlage}

Die Konzeption einer Belastung der Mitgliedstaaten entsprechend ihrer Wirtschaftskraft hat das Finanzierungssystem der Europäischen Gemeinschaften zu Beginn stark dominiert. Die Beitragsquoten im System der Finanzbeiträge orientierten sich an Sozialproduktsrelationen als Indikatoren der wirtschaftlichen Leistungsfähigkeit. Das System der Eigenmittel rückte von dieser Vorstellung zunächst ab, seit 1988 hat sich jedoch die Bedeutung des Bruttosozialproduktes wieder erhöht, da es als Maßstab für die vierte Finanzierungsquelle Eingang in das Finanzierungssystem gefunden hat. Für die Qualität dieser Eigenmittelart ist entscheidend, inwieweit es gelingt, in allen Mitgliedstaaten eine verläßliche und vergleichbare Ermittlung der Daten über das Brut- 


\section{Die Diskussion um einen angemessenen Finanzierungsanteil}

tosozialprodukt zu gewährleisten. ${ }^{17}$ Zwar werden die vom Statistischen Amt der Europäischen Gemeinschaften veröffentlichten Angaben einheitlich definiert, erfaßt und bewertet, die Technik der Ermittlung ist den Mitgliedstaaten jedoch freigestellt. ${ }^{18}$ Daran hat bisher auch die 1989 verabschiedete Richtlinie "Zur Harmonisierung der Erfassung des Sozialprodukts zu Marktpreisen" nichts geändert. 19

Durch die Kappung der Mehrwertsteuer-Bemessungsgrundlage bei 55\% des Bruttosozialprodukts eines Mitgliedslandes wurde versucht, den Einfluß des Gedankens einer Lastenverteilung entsprechend der wirtschaftlichen Leistungs fähigkeit zu verstärken. Bei den Mehrwertsteuer-Eigenmitteln fand eine anteilsmäßige Umschichtung zugunsten der Mitgliedstaaten statt, die einen relativ hohen Verbrauch aufwiesen. Dies waren 1988 Irland, Luxemburg und Großbritannien $^{20}$, seit 1989 kam zu diesen Staaten noch Portugal hinzu (siehe Tabelle 31 ).

Da durch die Kappung die Mehrwertsteuer-Bemessungsgrundlage insgesamt sinkt, können entsprechend nur noch weniger Ausgaben über die Mehrwertsteuer-Eigenmittel finanziert werden. Denn das Volumen der MehrwertsteuerEigenmittel wird ermittelt, indem auf die Mehrwertsteuer-Bemessungsgrundlage ein Satz von 1,4\% angewandt und von diesem Produkt der Bruttoausgleichsbetrag für Großbritannien abgezogen wird. ${ }^{21}$ Sinkt die Bemessungsgrundlage, sinkt daher auch das Volumen der Mehrwertsteuer-Eigenmittel. Insofern sinken die Mehrwertsteuer-Abführungen nicht nur bei den Mitgliedstaaten, die von der Kappung betroffen sind, sondern bei allen Mitgliedstaaten (siehe die negativen Differenzen in Tabelle 32). Die weitaus größte Reduzierung ist allerdings für Großbritannien festzustellen. Während die Mehrwertsteuer-Eigenmittel sinken, müssen bei einem gegebenen Haushaltsvolumen die Bruttosozialprodukt-Eigenmittel steigen.

17 Vgl. Messal $=$ Das neue Finanzierungssystem $=217$.

18 In Großbritannien werden beispielsweise die Sozialproduktsgrößen aus den Steuerstatistiken abgeleitet, was zu relativ geringeren Ansätzen führt. Vgl. Ott =Verteilungswirkungen $=35$.

19 Vgl. Rat der EG =Erfassung des BSPMP 1989=26ff.

20 Vgl. Europäisches Parlament $=$ Haushaltsplan $1989=105$.

21 Vgl. C. III. 1. b) ee). 
Tabelle 32: Die Bruttosozialprodukt(BSP)-Eigenmittel vor und nach der Kappung der Mehrwertsteuer (MWSt)Bemessungsgrundlage für das Haushaltsjahr 1989

\begin{tabular}{|c|c|c|c|c|c|c|}
\hline $\begin{array}{l}\text { Mit- } \\
\text { glied- } \\
\text { staat }\end{array}$ & $\begin{array}{l}\text { hypothetische } \\
\text { MWSt-Abfüh- } \\
\text { rung vor Kap- } \\
\text { pung1) } \\
\text { in Mio. ECU }\end{array}$ & $\begin{array}{l}\text { hypothetische } \\
\text { MWSt-Abfüh- } \\
\text { rung nach } \\
\text { Kappung2) } \\
\text { in Mio. ECU }\end{array}$ & \multicolumn{2}{|c|}{ Differenz } & $\begin{array}{c}\text { BSP-Eigen- } \\
\text { mittel vor } \\
\text { Kappung } \\
\text { in Mio. ECU }\end{array}$ & $\begin{array}{c}\text { BSP-Eigen- } \\
\text { mittel nach } \\
\text { Kappung } \\
\text { in Mio. ECU }\end{array}$ \\
\hline B & 770,19 & 766,75 & $-3,44$ & 0,54 & 102,14 & 122,64 \\
\hline DK & 532,72 & 530,34 & $-2,38$ & 0,37 & 70,43 & 84,57 \\
\hline D & 6721,20 & 6691,21 & $-29,99$ & 4,69 & 806,96 & 96,85 \\
\hline $\mathrm{E}$ & 1961,42 & 1952,67 & $-8,75$ & 1,37 & 226,90 & 272,46 \\
\hline F & 5596,72 & 5571,75 & $-24,97$ & 3,90 & 633,68 & 760,90 \\
\hline GRL & 295,24 & 293,92 & $-1,32$ & 0,21 & 40,25 & 48,37 \\
\hline IRL & 189,98 & 167,28 & $-22,70$ & 3,55 & 18,72 & 22,00 \\
\hline I & 3927,97 & 3910,45 & $-17,53$ & 2,74 & 572,41 & 687,33 \\
\hline LUX & 64,18 & 54,82 & $-9,36$ & 1,46 & 6,00 & 7,21 \\
\hline NL & 1257,98 & 1252,37 & $-5,61$ & 0,88 & 150,55 & 180,78 \\
\hline $\mathbf{P}$ & 320,91 & 257,95 & $-62,96$ & 9,84 & 28,25 & 33,92 \\
\hline UK & 5297,63 & 4846,91 & $-450,72$ & 70,45 & 530,78 & 637,33 \\
\hline Summe & 26936,14 & 26296,41 & $-639,73$ & 100,00 & 3186,58 & 3826,31 \\
\hline
\end{tabular}


Das bedeutet, daß sich durch die Kappung das Gewicht der Bruttosozialprodukt-Eigenmittel erhöht.

Bei den Mitgliedstaaten, die von der Kappung profitieren, nähert sich der Anteil an den Mehrwertsteuer-Eigenmitteln und den Bruttosozialprodukt-Eigenmitteln (Finanzierungsanteil), der vorher deutlich über dem Anteil am Bruttosozialprodukt lag, dem Bruttosozialprodukt-Anteil an. Bei einem Teil der übrigen Mitgliedstaaten (Italien, Belgien, Dänemark, Griechenland und die Niederlande), deren Finanzierungsanteil unter dem Bruttosozialprodukt-Anteil lag, steigt der Finanzierungsanteil und nähert sich damit ebenfalls dem Bruttosozialprodukt-Anteil an. Für die Bundesrepublik Deutschland ergibt sich dagegen der Effekt, daß die Kappung den Finanzierungsanteil, der zunächst unter dem Bruttosozialprodukt-Anteil lag, über den Bruttosozialprodukt-Anteil hinausschiebt. Für Frankreich und Spanien liegt die Situation vor, daß der Finanzierungsanteil, der schon über dem Bruttosozialprodukt-Anteil lag, noch weiter erhöht wurde (siehe Tabelle 33). Bei diesen Mitgliedstaaten ist insofern keine stärkere Orientierung am Bruttosozialprodukt festzustellen.

Die Tendenz, die gesamten Finanzierungsanteile nur noch (oder doch zumindest hauptsächlich) anhand des Bruttosozialproduktes zu beurteilen und das bestehende Einnahmensystem entsprechend anzupassen, hat im Falle der Kappung der Mehrwertsteuer-Bemessungsgrundlage lediglich zu einer weiteren Komplizierung des Einnahmensystems geführt, ohne daß die Zielsetzung einer stärkeren Orientierung am Bruttosozialprodukt durchgängig erreicht werden konnte. Es stellt sich angesichts dieser Entwicklung die Frage, ob es nicht sinnvoller wäre, zu einfachen und durchschaubaren Finanzbeiträgen, die sich an Sozialproduktschlüsseln orientieren, zurückzukehren. Ist dies nicht erwünscht, da wirkliche eigene Mittel für die Gemeinschaften angestrebt werden, darf das Einnahmensystem nicht allein nach dem Kriterium der Bruttosozialprodukt-Anteile beurteilt werden. 
Tabelle 33: Belastungs- und Entlastungswirkungen durch die Kappung in den einzelnen Mitgliedstaaten im Haushaltsjahr 1989

\begin{tabular}{|c|c|c|c|c|c|c|}
\hline $\begin{array}{l}\text { Mit- } \\
\text { glied- } \\
\text { staat }\end{array}$ & $\begin{array}{r}\text { Anteile am } \\
\text { BSP }\end{array}$ & $\begin{array}{l}\text { Abführung der } \\
\text { MWSt- und BSP- } \\
\text { Eigenmittel vor } \\
\text { der Kappung } \\
\text { in Mio. ECO }\end{array}$ & in $\%$ & $\begin{array}{l}\text { Abfuihrung der } \\
\text { MWSt- und BSP- } \\
\text { Eigenmittel nach } \\
\text { der Kappung } \\
\text { in Mio. ECO }\end{array}$ & in $\%$ & in Mio. ECU \\
\hline B & 3,21 & 872,33 & 2,90 & 889,40 & 2,95 & 17,06 \\
\hline DK & 2,21 & 603,15 & 2,00 & 614,90 & 2,04 & 11,75 \\
\hline D & 25,32 & 7528,06 & 24,99 & 7660,06 & 25,43 & 132,00 \\
\hline E & 7,12 & 2188,32 & 7,26 & 2225,12 & 7,39 & 36,80 \\
\hline F & 19,89 & 6230,40 & 20,68 & 6332,65 & 21,02 & 102,25 \\
\hline GRL & 1,26 & 335,49 & 1,11 & 342,26 & 1,14 & 6,77 \\
\hline IRL & $\mathbf{0 , 5 7}$ & 208,30 & 0,69 & 189,28 & 0,63 & $-19,01$ \\
\hline I & 17,96 & 4500,38 & 14,94 & 4597,78 & 15,26 & 97,40 \\
\hline LUX & 0,19 & 70,18 & 0,23 & 62,03 & 0,21 & $-8,15$ \\
\hline NL & 4,72 & 1408,53 & 4,68 & 1433,14 & 4,76 & 24,61 \\
\hline $\mathbf{P}$ & 0,89 & 349,15 & 1,16 & 291,87 & 0,97 & $-57,28$ \\
\hline UK & 16,66 & 5828,41 & 19,35 & 5484,24 & 18,21 & $-344,17$ \\
\hline Summel) & 100,00 & 30122,73 & 100,00 & 30122,73 & 100,00 & 0,00 \\
\hline $\begin{array}{l}\text { 1) } \\
\text { BSP } \\
\text { MWSt }\end{array}$ & $\begin{array}{l}\text { uttosozial } \\
\text { ehrwertst }\end{array}$ & $\begin{array}{l}\text { keiten sind durch } \\
\text { dukt }\end{array}$ & 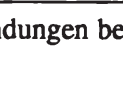 & & & \\
\hline
\end{tabular}




\section{Zur Regressivität der Mehrwertsteuer-Eigenmittel}

Die Tatsache, daß das Sozialprodukt und nicht der Verbrauch eines Mitgliedstaates als Indikator der wirtschaftlichen Leistungsfähigkeit betrachtet wird, bildet auch den Hintergrund für die Frage, ob die Mehrwertsteuer-Eigenmittel regressive Effekte aufweisen. Wenn den Mehrwertsteuer-Einnahmen der Europäischen Gemeinschaften ein regressiver Effekt unterstellt wird ${ }^{22}$, wird auf die Einkommensregressivität abgestellt, bei der mit steigendem Einkommen die prozentuale Steuerbelastung abnimmt. Bei den nationalen Verbrauchsteuern, etwa der deutschen Umsatzsteuer, kann ein solcher Effekt durch die mit wachsendem Einkommen sinkende Konsumquote auftreten. Auch wenn häufig versucht wird, diesen Effekt durch die Differenzierung der Steuersätze abzumildern, z. B. durch eine niedrigere Belastung des Grundbedarfs, bleibt zumindest im Bereich höherer Einkommen die regressive Wirkung bestehen. ${ }^{23}$

Während man bei der personellen Verteilungswirkung einer nationalen Steuer die Steuerbelastung auf die Einkommen der einzelnen Wirtschaftssubjekte bezieht, greift eine Inzidenzanalyse, die auf den zwischenstaatlichen Vergleich der Mitgliedstaaten abstellt, auf Volkseinkommensgrößen zurück. Die Begründung für eine vermutete Regressionswirkung der an die Europäischen Gemeinschaften abgeführten Mehrwertsteuer-Eigenmittel müßte sich in diesem Fall auf eine mit steigendem Volkseinkommen sinkende nationale Konsumquote stützen. "Da sich die Mehrwertsteuerabführungen allein an der Bemessungsgrundlage ausrichten, können ermäßigte Steuersätze und Steuerbefreiungen verschiedener Güter keinen Einfluß auf die Verteilungswirkungen ausüben." 24

Die Daten der Tabelle 34 zeigen, daß der für individuelle Einkommen im schichtenspezifischen Durchschnitt empirisch nachweisbare Sachverhalt sinkender marginaler Konsumquoten bei wachsenden Einkommen nicht so einfach auf den zwischenstaatlichen Bereich übertragen werden kann. Der private Verbrauch in den Mitgliedstaaten variiert keineswegs durchgehend invers mit der Höhe des Bruttoinlandsprodukt-pro-Kopf. Trotz der teilweise großen Unterschiede in den nationalen Bruttoinlandsprodukten-pro-Kopf liegen die Konsumquoten auf einem relativ einheitlichen Niveau. Sie lassen auch in ihrer

22 Vgl. u. a. Strasser $=$ Finanzen Europas $=125 \mathrm{f}$.

23 Vgl. Pohmer $=$ Allgemeine Umsatzsteuern $=706$.

24 Ott $=$ Verteilungswirkungen $=73$. 
Tabelle 34: Verwendung des Bruttoinlandsproduktes in den Mitgliedstaaten im Jahre 1988 in \% des Bruttoinlandsproduktes zu Marktpreisen

\begin{tabular}{|lrrrrrr|}
\hline $\begin{array}{l}\text { Mitglied- } \\
\text { staat }\end{array}$ & $\begin{array}{r}\text { Privater } \\
\text { Verbrauch }\end{array}$ & $\begin{array}{r}\text { Staats- } \\
\text { verbrauch }\end{array}$ & $\begin{array}{r}\text { Bruttoan- } \\
\text { lageinvesti- } \\
\text { tionen }\end{array}$ & $\begin{array}{r}\text { Vorratsver- } \\
\text { änderung }\end{array}$ & $\begin{array}{r}\text { Ausfuhr- } \\
\text { Einfuhr }\end{array}$ & $\begin{array}{r}\text { Bruttoinlands-do } \\
\text { produkt-pro } \\
\text { Kopf in \% des } \\
\text { Gemeinschafts- } \\
\text { durchschnitts }\end{array}$ \\
\hline LUX & 57,6 & 16,2 & 22,5 & $+1,3$ & $+2,3$ & 126,4 \\
D & 61,1 & 13,0 & 19,8 & $+0,7$ & $+5,1$ & 112,8 \\
DK & 54,1 & 25,8 & 17,6 & $-0,5$ & $+3,0$ & 110,1 \\
F & 60,4 & 18,8 & 20,0 & $+0,7$ & $+0,1$ & 108,7 \\
UK & 63,3 & 20,2 & 18,2 & $+0,2$ & $-3,6$ & 106,1 \\
I & 61,7 & 16,5 & 20,1 & $+1,1$ & $+0,7$ & 104,7 \\
NL & 60,0 & 15,5 & 21,2 & $-0,2$ & $+3,5$ & 103,6 \\
B & 63,3 & 15,7 & 18,0 & $+0,1$ & 3,1 & 100,8 \\
E & 62,8 & 14,3 & 22,5 & $+1,4$ & $-0,9$ & 75,1 \\
IRL & 57,2 & 17,0 & 17,3 & $-1,0$ & $+1,4$ & 64,2 \\
GRL & 66,7 & 20,6 & 18,1 & $+0,9$ & $-6,5$ & 54,2 \\
P & 58,5 & 14,3 & 28,2 & $+0,9$ & $-9,3$ & 53,9 \\
\hline
\end{tabular}

Quelle: Statistisches Bundesamt $=$ Ausland $1990=159 \mathrm{ff}$. und eigene Berechnungen. 
Differenzierung kaum einen Einfluß des Bruttoinlandsprodukts erkennen. Selbst wenn neben dem privaten Konsum der Staatsverbrauch berücksichtigt wird, läßt sich keine eindeutige Beziehung ableiten. ${ }^{25}$

Doch auch wenn dies nicht der Fall wäre, muß beachtet werden, daß der Tatbestand, der dann als "regressiv" bezeichnet würde, nichts mit einer regressiven personellen Belastung der Bürger zu tun hat; denn wie die innerstaatliche Verteilung der Abgabenlasten gestaltet wird, bleibt unbeantwortet. Bei der Diskussion um die "Regressivität" der Mehrwertsteuer-Eigenmittel zeigt sich ebenfalls die Tendenz, das gesamte Einnahmensystem anhand von Sozialproduktsgrößen zu beurteilen. Es ist widersprüchlich, den Verbrauch als Indikator für die Zahlungsverpflichtung festzulegen - wobei eigentlich immer eine wirkliche Beteiligung an den Mehrwertsteuer-Einnahmen der Mitgliedstaaten vorgesehen war, auch wenn diese nie verwirklicht wurde -, die Zahlungsverpflichtungen dann aber anhand von Sozialproduktsgrößen zu beurteilen. Wenn man dieses Kriterium so stark in den Vordergrund stellt, müßte man im Grunde wieder zu genauen Finanzbeiträgen nach dem Bruttosozialprodukt zurückkehren.

25 So auch Ott $=$ Verteilungswirkungen $=73 \mathrm{ff}$. 


\section{Zum Finanzausgleich im engeren Sinne}

Hinsichtlich des Finanzausgleichs i. e. S., bei dem es um eine Umverteilung zwischen den Gebietskörperschaften gleicher Ebene geht, läßt sich in den Europäischen Gemeinschaften von einem "Finanzausgleich mit partieller Ausgleichsorientierung"1 sprechen. Ein gezielter Einnahmenausgleich fehlt zwar, doch gehen Ansätze zur Umverteilung von der Ausgabenseite, vor allem von den Strukturfonds aus. In den letzten Jahren hat die Zielsetzung "Ausgleich zwischen den Mitgliedstaaten" sogar zunehmend an Bedeutung gewonnen.

Dies ist auf mehrere Ursachen zurückzuführen. ${ }^{2}$ In der zunehmenden wirtschaftlichen Integration wird vielfach die Notwendigkeit eines Ausgleichs gesehen. Es wird vermutet, daß die peripheren Staaten der Gemeinschaft von der Verwirklichung des Binnenmarktes weniger profitieren als die Kernländer, so daß sich die Disparitäten innerhalb der Europäischen Gemeinschaft erhöhen. ${ }^{3}$ Dieser Ansicht kann entgegengehalten werden, daß bei größerer Freizügigkeit der Produktionsfaktoren die regionalen Entwicklungsunterschiede über Preisund Kostendifferentiale ausgeglichen werden können. ${ }^{4}$ Die Kommission verweist dagegen auf bestehende Disparitäten. Sie hält diese für zu groß und die Dauer des Anpassungsprozesses für zu lang und fordert daher einen Ausgleich. ${ }^{5}$ Daneben ist zu berücksichtigen, daß sich die Stimmenverhältnisse in den Europäischen Gemeinschaften mit zunehmender Erweiterung zugunsten der weniger entwickelten Mitgliedstaaten verschoben haben, die ein großes Interesse an einem Ausgleich haben. Schließlich wird als Argument für den Ausgleich angeführt, daß die Entwicklungsunterschiede zwischen den Volkswirtschaften der Europäischen Gemeinschaft durch die Süderweiterungen ein solches Ausmaß angenommen haben, daß sie tiefverwurzelten Vorstellungen von sozialer Gerechtigkeit widersprechen.

Die stärkere Betonung des Ausgleichsziels hat dazu geführt, daß die Kommission versuchte, die fehlenden Ausgleichsmöglichkeiten auf der Einnahmenseite

$1 \quad$ Biehl $=$ Finanzausgleich IV $=692$.

2 Vgl. dazu u. a. Schäfers, Starbatty $=$ Kohäsion $=5 \mathrm{f}$.

3 So auch eine Studie des IFO-Instituts, vgl. Nam u. a. =Wettbewerbsfähigkeit $=11$.

4 Einen guten Überblick über beide Positionen bieten Krieger, Thoroe, Weskamp $=$ Regionales Wirtschaftswachstum $=7 \mathrm{ff}$.

5 Vgl. Krieger-Boden = Regionalpolitik der $E G=94 \mathrm{f}$. 


\section{Zum Finanzausgleich im engeren Sinne}

dadurch zu kompensieren, daß Aufgabenbereiche, die nicht primär dem Umverteilungsziel dienten, stärker in dieses einbezogen wurden (so z. B die Technologiepolitik). Die verstärkte Forderung nach einem zwischenstaatlichen Ausgleich fand ihren Niederschlag jedoch vor allem in der Aufstockung der Strukturfonds in den letzten Jahren. Obwohl die Strukturfonds nicht auf die Mitgliedstaaten als Ganzes, sondern (insbesondere beim Regionalfonds) auf die einzelnen Regionen in der Europäischen Gemeinschaft ausgerichtet sind, wird ein Ausgleich insofern erreicht, als die Europäische Gemeinschaft die Mitgliedstaaten, die nicht in der Lage sind, ihren strukturpolitischen Aufgaben in ausreichendem Maße nachzukommen, höhere Mittel zukommen läßt.

Auch wenn man akzeptiert, daß eine Umverteilung zwischen den Mitgliedstaaten notwendig ist, so stellt sich doch die Frage, ob die Strukturfonds die geeigneten Instrumente sind. Bei den kritischen Anmerkungen zu den Strukturfonds ${ }^{6}$ wurde deutlich, daß die allokationspolitischen Zielsetzungen der Strukturfonds nicht völlig von den Umverteilungszielsetzungen überlagert werden dürfen. Wenn die Umverteilung zu sehr dominiert, werden bei den Empfängern die Anreize gering sein, sich selbst zu helfen. Die Strukturpolitik der Europäischen Gemeinschaften darf nicht dazu mißbraucht werden, die Fehler nationaler Politik auszubügeln. Sie sollte insofern nur unterstützend eingesetzt werden. Durch allgemeine Zuweisungen würden Investitionsentscheidungen der Regionen nicht verzerrt, die Transparenz hinsichtlich der Nutznießer der Maßnahmen könnte erhöht werden und eine zielgerechtere Ausgestaltung der Maßnahmen wäre möglich. ${ }^{7}$

Die Aufstockung der Mittel der Strukturfonds ist jedoch auch vor dem Hintergrund zu sehen, daß die Verteilungswirkungen der Agrarmarktpolitik als unerwünscht angesehen werden und die Strukturfonds hier einen Ausgleich schaffen sollen.

Die Verteilungswirkungen der Agrarmarktausgaben und der Ausgaben der Strukturfonds sollen im folgenden aufgezeigt werden. Als Maßstab für den Wohlstand eines Mitgliedstaates wird dabei das Bruttoinlandsprodukt-pro-

6 Vgl. Abschnitt D. I. 3. b).

7 Vgl. Krieger-Boden $=$ Regionalpolitik der $\mathrm{EG}=96$. 
Kopf $\mathrm{f}^{\mathbf{6}}$ herangezogen, und zwar bezogen auf den Gemeinschaftsdurchschnitt, um den Vergleich zwischen den Mitgliedstaaten zu erleichtern. Ein Ausgleichseffekt liegt dann vor, wenn Mitgliedstaaten, die hinsichtlich ihres Bruttoinlandsprodukt-pro-Kopf unter dem Gemeinschaftsdurchschnitt liegen, verhältnismäßig höhere Zuflüsse aus Ausgaben erhalten. ${ }^{9}$ Verhältnismäßig (oder proportional) bedeutet dabei, daß die Anteile der Mitgliedstaaten an den Ausgaben ins Verhältnis zu den Anteilen am Bruttoinlandsprodukt der Gemeinschaft gesetzt werden, so daß überproportionale (unterproportionale) Zuflüsse bedeuten, daß der Anteil der Zuflüsse eines Mitgliedstaates aus den gesamten Ausgaben der Europäischen Gemeinschaften für eine bestimmte Politik höher (geringer) ist als sein Anteil am Bruttoinlandsprodukt.

Bei den Ausgaben des Europäischen Ausgleichs- und Garantiefonds für die Landwirtschaft-Garantie läßt sich kein einheitlicher Effekt ablesen (siehe Tabelle 35). Zwar erhalten einige Mitgliedstaaten, deren Bruttoinlandsproduktpro-Kopf deutlich unter dem Gemeinschaftsdurchschnitt liegt (Irland und Griechenland), überproportionale Zuflüsse, aber auch in Mitgliedstaaten mit überdurchschnittlichem Bruttoinlandsprodukt-pro-Kopf fließen überproportional viele Mittel. Dies gilt für die Niederlande, Dänemark und Frankreich. Unterproportionale Zuflüsse erhält neben wohlhabenden Staaten wie Deutschland und Großbritannien auch Portugal, das den niedrigsten Wert beim Bruttoinlandsprodukt-pro-Kopf aufweist. Insgesamt sind die Effekte also sehr uneinheitlich. Eine systematische Umverteilung zugunsten der weniger wohlhabenden Staaten läßt sich ebensowenig feststellen wie eine Umverteilung zugunsten der wohlhabenden Staaten.

Ein etwas anderes Bild ergibt sich bei der Abteilung Ausrichtung des Agrarfonds. Hier erhalten die meisten Mitgliedstaaten, deren Bruttoinlandsproduktpro-Kopf unter dem Gemeinschaftsdurchschnitt liegt, verhältnismäßig hohe und die meisten Mitgliedstaaten mit überdurchschnittlichem Bruttoinlandsprodukt-pro-Kopf nur verhältnismäßig geringe Zuflüsse. Allerdings gibt es auch

8 Obwohl das Bruttosozialprodukt insofern der geeignetere Indikator für den Wohlstand eines Landes ist, als die im Ausland erzielten Einkommen der Bürger einbezogen und die entsprechenden Einkommenströme, die an die übrige Welt geflossen sind, abgesetzt werden, wird hier auf das Bruttoinlandsprodukt zurückgegriffen, da das Bruttosozialprodukt häufig erst mit großer zeitlicher Verzögerung zur Verfügung steht und statistische Veröffentlichungen der Europäischen Gemeinschaft fast ausschließlich auf das Bruttoinlandsprodukt abstellen. Vgl. dazu auch Ott $=$ Verteilungswirkungen $=35$.

9 Vgl. dazu auch Henke $=$ Finanzierung der $E G=41$. 
Tabelle 35: Verteilungswirkungen der Ausgaben des Europäischen Ausgleichs- und Garantiefonds für die Landwirtschaft im Jahr 1988

\begin{tabular}{|c|c|c|c|c|c|c|}
\hline $\begin{array}{l}\text { Mit- } \\
\text { glied- } \\
\text { staat }\end{array}$ & $\begin{array}{l}\text { BIP-pro-Kopf } \\
\text { in \% des EG- } \\
\text { Durchschnitts }\end{array}$ & $\begin{array}{r}\text { BIP-Anteile } \\
\text { am BIP der } \\
\text { EG in \% } \\
\text { (2) }\end{array}$ & $\begin{array}{c}\text { Anteile an } \\
\text { den Ausgaben } \\
\text { des EAGFL- } \\
\text { Garantie } \\
\text { (3) }\end{array}$ & $\begin{array}{r}\text { Sp3:Sp2 } \\
\text { (4) }\end{array}$ & $\begin{array}{r}\text { Anteile an } \\
\text { den Ausgaben } \\
\text { des EAGFL- } \\
\text { Ausrichtung } \\
\text { (5) }\end{array}$ & Sp5:Sp2 \\
\hline LU & 126,4 & 0,15 & 0,0 & - & 0,2 & 1,33 \\
\hline D & 112,8 & 21,4 & 17,1 & 0,80 & 11,5 & 0,54 \\
\hline DK & 110,1 & 1,7 & 4,4 & 2,59 & 1,2 & 0,76 \\
\hline F & 108,7 & 18,7 & 22,8 & 1,22 & 24,6 & 1,32 \\
\hline UK & 106,1 & 18,6 & 7,0 & 0,38 & 7,4 & 0,40 \\
\hline I & 104,7 & 18,5 & 15,6 & 0,84 & 17,5 & 0,95 \\
\hline NL & 103,6 & 4,7 & 14,3 & 3,04 & 0,4 & 0,09 \\
\hline B & 100,8 & 3,1 & 2,7 & 0,87 & 1,6 & 0,52 \\
\hline $\mathrm{E}$ & 75,1 & 9,0 & 6,7 & 0,74 & 7,9 & 0,88 \\
\hline IRL & 64,2 & 0,7 & 3,8 & 5,43 & 7,2 & 10,29 \\
\hline GRL & 54,2 & 1,7 & 5,0 & 2,94 & 11,7 & 6,88 \\
\hline P & 53,9 & 1,6 & 0,6 & 0,38 & 8,8 & 5,50 \\
\hline
\end{tabular}

Quelle: Statistisches Bundesamt $=$ Ausland 1988 $=100$ und Europäischer Rechnungshof $=$ Jahresbericht $1988=$ sowie eigene Berechnungen. 
hier einige Ausnahmen. So erhält der wohlhabende Mitgliedstaat Frankreich überproportional viele Mittel aus der Abteilung Ausrichtung, während nach Spanien, dessen Bruttoinlandsprodukt-pro-Kopf unter dem Gemeinschaftsdurchschnitt liegt, nur unterproportional viele Mittel fließen. Trotz dieser Ausnahmen läßt sich eine gewisse Tendenz zur Umverteilung ablesen. Allerdings kann auch bei der Abteilung Ausrichtung des Agrarfonds nicht von einem systematischen Instrument zwischenstaatlicher Umverteilung gesprochen werden. ${ }^{10}$

Bei den Ausgaben des Europäischen Fonds für regionale Entwicklung erhält man ein eindeutigeres Ergebnis (siehe Tabelle 36). Hier fließen überproportional hohe Zahlungen in alle weniger wohlhabenden Mitgliedstaaten, während die meisten Mitgliedstaaten, deren Bruttoinlandsprodukt-pro-Kopf über dem Gemeinschaftsdurchschnitt liegt, verhältnismäßig niedrige Beträge bekommen. Die Ausnahme bilden hier nur Luxemburg, Großbritannien und Italien, die leicht höhere Anteile an den Ausgaben des Europäischen Fonds für regionale Entwicklung erhalten als es ihrem Anteil am Bruttoinlandsprodukt der Gemeinschaft entspricht. Insgesamt läßt sich hier also eine Umverteilungstendenz ablesen. Dies gilt auch für den Europäischen Sozialfonds, bei dem sich für die wohlhabenden Mitgliedstaaten nur eine Ausnahme, nämlich Großbritannien, ergibt, das überproportionale Zuflüsse erhält.

Die Strukturfonds weisen also durchaus eine Tendenz zur Umverteilung auf, wenngleich diese bei den einzelnen Fonds immer wieder durchbrochen wird (siehe Tabelle 37). Dieser Umverteilungseffekt wird bei der Betrachtung der gesamten zurechenbaren Ausgaben allerdings durch die Verteilungseffekte der Ausgaben der Abteilung Garantie des Europäischen Agrarfonds überlagert. Dennoch ist festzuhalten, daß auch bei den gesamten zurechenbaren Ausgaben all diejenigen Mitgliedstaaten, deren Bruttoinlandsprodukt-pro-Kopf unter dem Gemeinschaftsdurchschnitt liegt, überproportional hohe Mittelzuflüsse erhalten. Dies gilt jedoch ebenfalls für die "reichen Agrarstaaten" wie die Niederlande, Dänemark und Frankreich, so daß insgesamt kein einheitlicher Effekt festgestellt werden kann.

10 Vgl. dazu auch Ott $=$ Verteilungswirkungen $=202$. 
Tabelle 36: Die Verteilungswirkungen der Ausgaben des Europäische Fonds für regionale Entwicklung und des Europäischen Sozialfonds im Jahre 1988

\begin{tabular}{|c|c|c|c|c|c|c|}
\hline \multirow{2}{*}{$\begin{array}{l}\text { Mit- } \\
\text { glied- } \\
\text { staat }\end{array}$} & \multirow{2}{*}{$\begin{array}{l}\text { BIP-pro-Kopf } \\
\text { in \% des EG- } \\
\text { Durchschnitts } \\
\text { (1) }\end{array}$} & \multirow{2}{*}{$\begin{array}{r}\text { BIP-Anteile } \\
\text { EG in \% } \\
(2)\end{array}$} & \multirow{2}{*}{$\begin{array}{l}\text { Anteile an } \\
\text { den Ausgaben } \\
\text { des EFRE } \\
\text { (3) }\end{array}$} & \multicolumn{2}{|c|}{ Sp3:Sp2 $\begin{array}{r}\text { Anteile an } \\
\text { den Ausgaben }\end{array}$} & \multirow{2}{*}{$\begin{array}{r}\text { Sp5:Sp2 } \\
\text { (6) }\end{array}$} \\
\hline & & & & (4) & (5) & \\
\hline LUX & 126,4 & 0,15 & 0,2 & 1,33 & - & - \\
\hline D & 112,8 & 21,4 & 3,1 & 0,54 & 6,4 & 0,30 \\
\hline DK & 110,1 & 1,7 & 0,3 & 0,18 & 1,5 & 0,88 \\
\hline F & 108,7 & 18,7 & 14,1 & 0,75 & 12,7 & 0,68 \\
\hline UK & 106,1 & 18,6 & 18,7 & 1,01 & 20,8 & 1,12 \\
\hline I & 104,7 & 18,5 & 19,3 & 1,04 & 14,3 & 0,77 \\
\hline NL & 103,6 & 4,7 & 0,4 & 0,09 & 2,0 & 0,43 \\
\hline B & 100,8 & 3,1 & 1,6 & 0,52 & 1,4 & 0,45 \\
\hline $\mathrm{E}$ & 75,1 & 9,0 & 17,6 & 1,96 & 17,7 & 1,97 \\
\hline IRL & 64,2 & 0,7 & 4,4 & 6,29 & 7,7 & 11,00 \\
\hline GRL & 54,2 & 1,7 & 10,1 & 5,94 & 6,4 & 3,80 \\
\hline $\mathbf{P}$ & 53,9 & 1,6 & 10,7 & 6,69 & 8,8 & 5,50 \\
\hline
\end{tabular}

Quelle: Statistisches Bundesamt $=$ Ausland $1988=100$ und Europäischer Rechnungshofess $=$ Jahresbericht $1988=$ sowie eigene Berechnungen. 
Tabelle 37: Die Ausgaben der Strukturfonds insgesamt und gesamte zurechenbare Ausgaben im Jahre 1988

\begin{tabular}{|c|c|c|c|c|c|c|}
\hline $\begin{array}{l}\text { Mit- } \\
\text { glied- } \\
\text { staat }\end{array}$ & $\begin{array}{l}\text { BIP-pro-Kopf } \\
\text { in \% des EG- } \\
\text { Durchschnitts }\end{array}$ & $\begin{array}{r}\text { BIP-Anteile } \\
\text { am BIP der } \\
\text { EG in \% } \\
\text { (2) }\end{array}$ & $\begin{array}{c}\text { Anteile an den } \\
\text { Ausgaben der } \\
\text { Strukturfonds } \\
\text { insgesamt } \\
\text { (3) }\end{array}$ & Sp3:Sp2 & $\begin{array}{r}\text { Anteile an den } \\
\text { zurechenbaren } \\
\text { Ausgaben } \\
\text { insgesamt } \\
\text { (5) }\end{array}$ & Sp5:Sp2 \\
\hline LUX & 126,4 & 0,2 & 0,2 & 1,0 & - & - \\
\hline D & 112,8 & 21,4 & 5,7 & 0,3 & 15,0 & 0,7 \\
\hline DK & 110,1 & 1,7 & 0,9 & 0,5 & 3,6 & 2,1 \\
\hline $\mathrm{F}$ & 108,7 & 18,7 & 15,5 & 0,8 & 20,3 & 1,1 \\
\hline UK & 106,1 & 18,6 & 17,5 & 0,9 & 9,0 & 0,5 \\
\hline I & 104,7 & 18,5 & 17,2 & 0,9 & 15,4 & 0,8 \\
\hline NL & 103,6 & 4,7 & 1,0 & 0,2 & 10,9 & 2,3 \\
\hline B & 100,8 & 3,1 & 1,2 & 0,4 & 2,3 & 0,7 \\
\hline $\mathrm{E}$ & 75,1 & 9,0 & 15,9 & 1,8 & 11,1 & 1,2 \\
\hline IRL & 64,2 & 0,7 & 6,1 & 8,7 & 4,1 & 5,9 \\
\hline GRL & 54,2 & 1,7 & 9,1 & 5,4 & 5,3 & 3,1 \\
\hline $\mathrm{P}$ & 53,9 & 1,6 & 9,7 & 6,1 & 2,5 & 1,6 \\
\hline
\end{tabular}

Quelle: Statistisches Bundesamt $=$ Ausland $1988=100$ und Europäischer Rechnungshof $=$ Jahresbericht $1988=$ sowie eigene Berechnungen. 
Insgesamt wird deutlich, daß die Verteilungseffekte der Agrarmarktpolitik nicht duch die Ausgaben der Strukturfonds kompensiert werden können. Es besteht die Gefahr, daß die Mittel der Strukturfonds mit diesem Argument, das nichts mehr mit der eigentlichen strukturpolitischen Zielsetzung zu tun hat, immer weiter aufgebläht werden.

Hinter dem Versuch, die unerwünschten Verteilungswirkungen der Agrarmarktpolitik durch die Strukturfonds auszugleichen, verbirgt sich wiederum die Tatsache, daß der Konsensrahmen in den Europäischen Gemeinschaften nicht vollständig ist. Dies hat nicht nur zur Folge, daß es keinen expliziten Einnahmenausgleich gibt, sondern auch, daß die Verteilungseffekte der Gemeinschaftsausgaben ${ }^{11}$ mißtrauisch beobachtet werden, wie die Forderung nach "juste retour" deutlich gezeigt hat. ${ }^{12}$ Diese Verteilungseffekte kann man als impliziten Finanzausgleich i. e. S. bezeichnen.

Gegen die Betrachtung der Verteilungseffekte der verschiedenen Ausgaben kann eingewandt werden, daß die einzelnen Bereiche der Politik nicht zu einer reinen Verteilungspolitik degenerieren dürfen, da sie eigene Ziele zu verwirklichen haben. ${ }^{13}$ Dies gilt insbesondere für Ausgaben im Rahmen der Europäischen Atomgemeinschaft oder auch für die Forschungspolitik. Aber gerade die Agrarpolitik soll ja eine Umverteilung zugunsten der Landwirtschaft bewirken. Sollten sich dabei unterschiedliche Verteilungseffekte z. B. zwischen Dänemark oder den Niederlanden einerseits und Griechenland andererseits ergeben, so beruht dies auf dem unterschiedlich hohen Grad an Protektion und Subventionierung. Gerade die Tatsache, daß beim gegenwärtig hohen Stand an Heterogenität und damit niedriger Solidarität formal direkte Einnahmenausgleichsregelungen nicht zu erwarten sind, darf kein Grund dafür sein, jeden beliebigen Verteilungseffekt in den Europäischen Gemeinschaften zu rechtfertigen. ${ }^{14}$ Nur ist es nicht sinnvoll, unerwünschte Verteilungswirkungen der Agrarpolitik mit allein aus diesem Grunde ansteigenden Strukturfondsausgaben ausgleichen zu wollen. Hier muß an der Agrarmarktpolitik selbst angesetzt werden.

11 Hierbei ist stets die Inzidenz der Zahlungsströme gemeint. Diese kann jedoch nur als erste Stufe einer weitergehenden Inzidenzanalyse betrachtet werden. Auf die Probleme einer regionalen Überwälzung gehen diese Überlegungen überhaupt nicht ein.

12 Vgl. dazu auch Biehl =EG-Finanzverfassung $=77$.

13 Vgl. Biehl, Winter $=$ EG-Finanzierung $=77$.

14 Vgl. Biehl, Winter $=$ EG-Finanzierung $=75 \mathrm{f}$. 


\section{E. SCHLUSSBETRACHTUNG}

Durch die europäische Integration ist aus der Sicht der Bundesrepublik Deutschland eine neue föderative Struktur entstanden. Diese Struktur wurde herausgearbeitet und anhand der Kriterien, die auf der Grundlage der Föderalismustheorie entwickelt wurden, kritisch überprüft. Die wichtigsten Ergebnisse sollen noch einmal kurz zusammengefaßt werden.

Die Europäischen Gemeinschaften haben die Zahl ihrer Aufgaben im Laufe der Zeit stark erhöht: Erstens sind Aufgaben, die die Gemeinschaften zunächst ohne rechtliche Grundlage an sich gezogen hatten, schließlich in den Verträgen verankert worden. Zweitens beeinflussen die Gemeinschaften durch die Harmonisierungsvorschriften im Rahmen der Vollendung des Binnenmarktes zunehmend nationale Aufgaben, für die sie auch heute noch keine vertraglichen Zuständigkeiten besitzen. Drittens ist festzustellen, daß auch die Intensität der gemeinschaftlichen Wahrnehmung von Aufgaben im Bereich konkurrierender Kompetenzen steigt.

Die Tatsache, daß die Europäischen Gemeinschaften zunehmend mehr Aufgaben an sich ziehen, schränkt den Spielraum von Bund, Ländern und Gemeinden bei der Wahrnehmung ihrer Aufgaben ein. Der Bund ist von der Verlagerung der meisten Aufgabengebiete auf die Europäischen Gemeinschaften betroffen. Doch kann er durch seinen Vertreter im Ministerrat die gemeinschaftliche Rechtsetzung mitgestalten. Dies bedeutet allerdings nicht, daß den Ländern keine Einflußmöglichkeiten auf die Rechtsetzung der Gemeinschaften verbleiben. Während sie ihren Einfluß zunächst vor allem auf informellem Wege geltend machen konnten, wurde er zunehmend auch institutionell abgesichert.

Selbst wenn die Europäischen Gemeinschaften Aufgaben an sich gezogen haben, besteht bei einem Teil der gemeinschaftlichen Rechtsetzung - den Richtlinien - für die nationalen Ebenen unter Umständen die Möglichkeit, Spielräume bei der Umsetzung der Richtlinien in nationales Recht zu nutzen. Außerdem bleiben den nationalen Ebenen bei der Ausführung von Rechtsakten der Europäischen Gemeinschaften ebenfalls noch gewisse - allerdings häufig begrenzte - Gestaltungsmöglichkeiten. Die grundsätzliche Zuordnung der Ausführungskompetenzen in der Bundesrepublik Deutschland auf die Länder bleibt 
auch bei der Ausführung von Rechtsakten der Europäischen Gemeinschaften erhalten.

Dem Subsidiaritätsprinzip wird bei der Aufgabenverteilung in den Europäischen Gemeinschaften nicht genügend Rechnung getragen. Dies wird besonders deutlich, wenn Aufgabenbereiche, die in der Bundesrepublik Deutschland bei den Ländern verblieben sind, auf die Gemeinschaften verlagert werden, denn auch der Aufgabenverteilung in der Bundesrepublik Deutschland liegt das Subsidiaritätsprinzip zugrunde.

Gegen eine zu weitgehende Zentralisierung von Aufgaben auf der Gemeinschaftsebene spricht vor allem die Tatsache, daß die Präferenzen in den Europäischen Gemeinschaften in vielen Bereichen noch sehr heterogen sind. Die Gefahr, daß eine zentrale Aufgabenerfüllung, die die regional unterschiedlichen Präferenzen nicht beachtet, in verstärktem Maße zu "frustrierten Minderheiten" führt, ist in den Europäischen Gemeinschaften besonders hoch.

Die heterogenen Präferenzen führen außerdem zu hohen Konsensfindungskosten in den Europäischen Gemeinschaften. Problematisch ist dabei die Praxis des "log-rolling", bei dem völlig unterschiedliche Sachverhalte zu einem "package-deal" zusammengeschnürt werden, um alle Beteiligten zufriedenzustellen. Korrekturen, die sich später unter Umständen als notwendig erweisen könnten, werden dadurch von vornherein unmöglich. Schließlich finden die hohen Konsensfindungskosten der Europäischen Gemeinschaften ihren Ausdruck auch in einem hohen Zeitaufwand, so z. B. bei den sogenannten Marathonsitzungen des Ministerrats und der teilweise Jahre dauernden Spanne zwischen Vorlage eines Rechtsaktes durch die Kommission und dessen Verabschiedung durch den Rat.

Schließlich ist grundsätzlich zu bemerken, daß die Bürger ihre unterschiedlichen Präferenzen aufgrund der politischen Distanz und der institutionellen Gegebenheiten in den Europäischen Gemeinschaften schlechter zur Geltung bringen können als auf nationaler Ebene.

Eine Verlagerung von Aufgaben auf die Gemeinschaft ist vor allem dann sinnvoll, wenn grenzüberschreitende Spillover-Effekte auftreten, die nicht durch bilaterale Verhandlungen kompensiert werden können, oder wenn durch die Bereitstellung öffentlicher Leistungen auf der Gemeinschaftsebene Skalenerträge genutzt werden können. 
Umweltpolitische Maßnahmen der Europäischen Gemeinschaften, die sich auf grundsätzliche Rahmenbedingungen - etwa die Beachtung des Verursacherprinzips - beziehen oder die der Bekämpfung multilateraler Umweltprobleme dienen, können so gerechtfertigt werden. Doch gibt es auch im Bereich der Umweltpolitik vielfältige Interessenunterschiede in der Gemeinschaft, die einer völligen Zentralisierung dieser Aufgaben entgegenstehen. Diese Interessenunterschiede schlagen sich nicht zuletzt in den Entscheidungen des Ministerrates nieder; dort werden nur Lösungen auf dem kleinsten gemeinsamen Nenner gefunden. Strengere Maßnahmen sind den Mitgliedstaaten zwar grundsätzlich erlaubt, allerdings nur dann, wenn sie nicht zu Wettbewerbsverzerrungen führen. Da im Grunde jede Umweltvorschrift Auswirkungen auf den Wettbewerb hat, muß entschieden werden, welche Störungen hingenommen werden können und welche nicht. Dabei ist zu berücksichtigen, daß unterschiedliche nationale Umweltvorschriften auch die Folge divergierender nationaler Immissionsgegebenheiten sein können, die als Standortfaktoren durchaus wettbewerbswirksam werden sollen. Produktbezogene Umweltnormen können dagegen im Binnenmarkt als nicht-tarifäre Handelshemmnisse betrachtet werden. Folgt man dieser Sichtweise, müßten umweltbedingte Produktanforderungen vollständig harmonisiert werden. Strengere nationale Vorschriften wären nicht möglich.

Dieser Konflikt zwischen nationaler Autonomie und Harmonisierung von Rechtsvorschriften durch die Europäischen Gemeinschaften ist ein generelles Problem bei der Verwirklichung des Binnenmarktes. Allerdings können Hemmnisse technischer Art nicht nur durch die Harmonisierung abgebaut werden, sondern auch dadurch, daß im Wege der gegenseitigen Anerkennung jedem Produkt, das in einem anderen Mitgliedstaat rechtmäßig hergestellt oder vertrieben wird, der Zugang zum heimischen Markt gestattet wird. Die Kommission wollte nach dem Prinzip verfahren, daß so wenig Harmonisierung wie möglich und so viel Harmonisierung wie nötig zu verwirklichen sei. Diesem Anspruch wird die Gemeinschaft in der Realität nicht gerecht. Einerseits zeigt ein Blick auf die Verkehrspolitik, daß notwendige Harmonisierungsmaßnahmen nicht immer getroffen werden. Andererseits zeigt sich bei Detailfragen eine starke Tendenz zur Überreglementierung. Die Tatsache, daß eine zentrale Aufgabenerfüllung sinnvoll sein kann, bedeutet nicht, daß damit jede Art und Weise der Aufgabenerfüllung gerechtfertigt werden kann. Die grundsätzlichen Vorteile des Binnenmarktes erlauben es nicht, jedes Maß an Harmonisierung von Rechtsvorschriften zu akzeptieren. 
Das Beispiel der Technologiepolitik macht deutlich, daß neben den Vorteilen einer Zentralisierung auch deren negative Wirkungen zu beachten sind, die bei der Technologiepolitik vor allem durch verminderten Wettbewerbsdruck entstehen. Die starke Ausweitung der Gemeinschaftskompetenzen auf diesem Gebiet läßt sich mit allokationspolitischen Argumenten nicht rechtfertigen. Da die gemeinschaftliche Technologiepolitik jedoch für die weniger entwickelten Mitgliedstaaten einen kostenlosen Technologietransfer bietet, dient sie auch verteilungspolitischen Zielsetzungen. Diese Vermischung der Ziele ist problematisch. Eine effiziente Technologiepolitik wird um so mehr gefährdet, je stärker der Einfluß der verteilungspolitischen Zielsetzung wird. Dabei ist der Erfolg des Technologietransfers in die Mitgliedstaaten mit Entwicklungsrückständen nicht von vornherein sicher. Gerade wenn Mitgliedstaaten in ihren Wirtschaftsstrukturen erhebliche Unterschiede aufweisen und unterschiedliche komparative Kostenvorteile besitzen, muß eine gemeinsame Forschung nicht sinnvoll sein.

Noch deutlicher im Spannungsverhältnis allokations- und verteilungspolitischer Zielsetzungen stehen die Regionalpolitik und die Agrarpolitik. Bei der Regionalpolitik müssen beide Zielsetzungen nicht von vornherein konfliktär sein; vielmehr kann es einen bestimmten Spielraum geben, innerhalb dessen durch regionalpolitische Maßnahmen sowohl das Wachstum der Europäischen Gemeinschaften insgesamt erhöht, als auch die regionalen Disparitäten vermindert werden können. Wird jedoch das Umverteilungsziel zu stark betont, besteht die Gefahr, daß das Wachstum in den entwickelteren Regionen gebremst wird und damit auch die zur Umverteilung zur Verfügung stehenden Mittel geringer ausfallen. Regionale Disparitäten werden sehr stark durch die nationale Wirtschaftspolitik bestimmt. Die Regionalpolitik der Gemeinschaft darf nicht dazu mißbraucht werden, nationale Fehler auszubügeln. Die Planung und Durchführung regionalpolitischer Maßnahmen sollte möglichst dezentral erfolgen; dies garantiert eine flexible, an die Probleme angepaßte Politik und einen interregionalen Wettbewerb.

Auch bei der Agrarpolitik ist das Umverteilungsziel immer stärker in den Vordergrund gerückt. Die Einkommenssicherung über Preisgarantien hat dazu geführt, daß der Preis seine Steuerungsfunktion verloren hat. Nahezu alle Probleme der Agrarpolitik sind auf diese Tatsache zurückzuführen. Direkte Einkommensübertragungen an die Landwirte, wie sie zahlreiche Reformvorschläge vorsehen, können sinnvoll nicht von der Gemeinschaftsebene geleistet 
werden, da sich die Angemessenheit der Lebenshaltung nur im Verhältnis zur Einkommenslage und zur Entwicklung des sozialen Umfeldes im jeweiligen Mitgliedstaat definieren läßt. Die starken Einkommensunterschiede in der Europäischen Gemeinschaft erfordern eine nationale Differenzierung des Zielsystems. Eine Rückverlagerung entsprechender Kompetenzen auf die Mitgliedstaaten ist sinnvoll.

Die Kompetenzverteilung im Rahmen der Stabilisierungspolitik hängt von der weiteren Entwicklung zur Wirtschafts- und Währungsunion ab. Trotz einer gewissen Konvergenz, die allerdings weitgehend auf Außeneinflüssen beruht, sind die Inflationsraten in den Mitgliedstaaten noch ziemlich unterschiedlich. Eine Verlagerung der geldpolitischen Kompetenzen auf den Ausschuß der Zentralbankpräsidenten birgt die Gefahr in sich, daß das im Europäischen Währungssystem durch die Ankerfunktion der D-Mark erzielte Niveau an Geldwertstabilität nicht beibehalten werden kann, sondern sich die unterschiedlichen geldpolitischen Ansichten in einem Kompromiß auf mittlerer Linie niederschlagen. Die Finanzpolitik, die auch auf längere Sicht in der Kompetenz der Mitgliedstaaten bleibt, wird bisher nicht in allen Mitgliedstaaten in gleichem Maße an stabilitätspolitischen Erfordernissen orientiert. So ist die Budgetpolitik der Mitgliedstaaten sehr unterschiedlich. Um eine höhere Konvergenz auf diesem Gebiet zu erreichen, können Maßnahmen zur Begrenzung öffentlicher Haushaltsdefizite sinnvoll sein. Sie müssen jedoch eindeutig festgelegt und auch durchgesetzt werden.

Die Aufgabenverteilung in den Europäischen Gemeinschaften verstößt in fast allen Bereichen gegen den Grundsatz der klaren Aufgabenzuordnung. Die wenigsten Aufgaben werden durch die Europäischen Gemeinschaften allein wahrgenommen; in der Regel werden die Gemeinschaften unterstützend und ergänzend zu nationalen Ebenen tätig. Dies führt zu Problemen der Kompetenzverflechtung: Die Verantwortlichkeiten werden verwischt, die öffentliche und die parlamentarische Kontrolle erschwert. Besondere Probleme ergeben sich dann, wenn von vornherein Zielkonflikte zwischen der nationalen und der gemeinschaftlichen Politik vorliegen, wie am Beispiel der Agrarpolitik deutlich wird. Die Probleme der laufenden Koordinierung gemeinschaftlicher und nationaler Aktivitäten wurden am Beispiel der Regionalpolitik aufgezeigt.

Der Gesamthaushalt der Europäischen Gemeinschaften zeigt, daß dieser für viele Politikbereiche nur einen (ordnungspolitischen) Rahmen setzt. Die Aus- 
gaben der Gemeinschaft konzentrieren sich auf bestimmte Bereiche, vor allem auf die Agrarmarktpolitik, die Strukturpolitik, die Forschungs- und Technologiepolitik sowie die Entwicklungshilfe. Diese Ausgaben der Europäischen Gemeinschaften lassen sich jedoch nicht nur nach Ausgabenarten unterscheiden, sondern auch danach, ob sie nationale Ausgaben ersetzen, ob sie diese ergänzen oder ob sie erst durch die Existenz der Gemeinschaften entstanden sind.

Die Ausgabenverteilung in den Europäischen Gemeinschaften weist eine ganze Reihe von Mängeln auf. Von vornherein problematisch ist die Einteilung der Ausgaben in obligatorische und nicht-obligatorische und die entsprechende Aufteilung der Ausgabenkompetenzen zwischen Rat und Parlament. Für beide Organe ist die Höhe der von ihnen gestaltbaren Ausgaben vor allem eine Frage der Gewichtung von Kompetenzen. Die Finanzierungsfrage haben beide Organe nicht direkt vor den Bürgern zu verantworten; daher versucht jedes Organ, seine Ausgaben möglichst zu erhöhen. Dies führt tendenziell zu einer Aufblähung des Gemeinschaftshaushalts.

Besondere Probleme für den Haushalt der Europäischen Gemeinschaften ergeben sich durch die hohen Agrarausgaben, die zu einem Hemmschuh für die Entwicklung anderer Ausgabenbereiche geworden sind. Auch hier ist das Verfahren der Ausgabenbewilligung für die Schwierigkeiten entscheidend. Die Agrarausgaben hängen vor allem von den Agrarpreisbeschlüssen ab, für die der Agrarministerrat verantwortlich ist. Dieser sucht in der Regel nur einen Ausgleich zwischen verschiedenen nationalen agrarpolitischen Positionen. Nicht berücksichtigt werden dagegen die Interessen der Verbraucher und der Steuerzahler, die bei diesen Entscheidungen auch nicht vertreten sind. Während in den einzelnen Mitgliedstaaten den Ausgabenwünschen der verschiedenen Ressorts durch den Finanzminister Grenzen gesetzt werden, hat der Finanzministerrat keine entsprechende Funktion. Die Agrarminister können sich insofern ihre Ausgaben selbst genehmigen. Die Agrarausgaben einzudämmen, ist jedoch auch deshalb schwierig, weil sie bei der bestehenden Agrarmarktordnung von einer Reihe exogener Faktoren abhängen, etwa den Weltmarktpreisen für landwirtschaftliche Produkte.

Die bisherigen Versuche, die Agrarmarktausgaben zu senken, waren nicht erfolgreich. Die Erzeugerabgaben haben nicht verhindert, daß die Ausgaben weiter ansteigen; die teilweise komplizierten Regelungen erhöhen lediglich den administrativen Aufwand und laden zum Betrug ein. Auch die Stabilisatoren 
stellen nur einen ersten Schritt zu einer am Markt orientierten Agrarpolitik dar: Die Preise werden weiterhin administrativ festgesetzt, und die Entscheidung, von welcher Menge an eine Preissenkung stattfindet, bleibt letztlich willkürlich.

Nach dem Konnexitätsprinzip müßte es den nationalen Ebenen mit zunehmendem finanziellem Engagement der Europäischen Gemeinschaften in der Agrarpolitik möglich sein, Kürzungen nationaler Agrarausgaben vorzunehmen. Dies gilt vor allem deshalb, weil die gemeinschaftliche Agrarpolitik nicht nur die nationale Politik unterstützen, sondern eigenständige Aufgaben wahrnehmen soll. Eine entsprechende Entlastungswirkung ist unter Berücksichtigung der Steuervergünstigungen und der sozialpolitischen Ausgaben für die Landwirtschaft in der Bundesrepublik Deutschland nicht festzustellen.

Dagegen finden sich gerade im Agrarbereich zunehmend Beschlüsse der Europäischen Gemeinschaften, die zu zusätzlichen Ausgaben auf nationaler Ebene führen, wie z. B. das Flächenstillegungsprogramm. In der Bundesrepublik Deutschland stellt sich die Frage, wie diese zusätzlichen Lasten, die auch in anderen Bereichen anfallen, auf Bund und Länder zu verteilen sind. Bei der Agrarstrukturpolitik tragen Bund und Länder die Ausgaben im Rahmen der Gemeinschaftsaufgabe gemeinsam. Eine allgemeine rechtliche Klärung der Frage, wie Belastungen durch Gemeinschaftsentscheidungen auf die nationalen Ebenen zuzuordnen sind, gibt es bisher nicht, dies muß jedesmal neu verhandelt werden.

Eine weitere Durchbrechung des Konnexitätsprinzips stellen die Mischfinanzierungen dar, wie sie etwa bei den Strukturfonds existieren. Sie bergen die Gefahr, daß der Investitionskalkül verfälscht wird und daß minder dringliche Vorhaben zuerst in Angriff genommen werden, nur weil dafür Mittel bereitstehen. Häufig werden Folgekosten nicht berücksichtigt. Die Tatsache, daß die Aufbringung eines Eigenanteils gefordert wird und Projekte vorfinanziert werden müssen, kann außerdem strukturkonservierend wirken. Die rückwirkende Erstattung und die Möglichkeit, daß Erstattungsanträge auch abgelehnt werden können, führen dazu, daß hier die Mitnahmeeffekte besonders hoch sind.

Das Einnahmensystem der Europäischen Gemeinschaften wurde mehrfach geändert, bis es die heutige Form erhielt. Neben den Zöllen und Agrarabschöpfungen als "originären" Eigenmitteln sind die wesentlichen Einnahmequellen der Europäischen Gemeinschaften die Mehrwertsteuer-Eigenmittel so- 


\section{Schlußbetrachtung}

wie die Bruttosozialprodukt-Eigenmittel. Die Einnahmenautonomie der Europäischen Gemeinschaften ist nicht allzu groß. Bei den Zöllen und Agrarabschöpfungen besitzen sie zwar die alleinige Objekt- und Ertragshoheit. Da diese Einnahmen vor allem handels- und agrarpolitischen Zielen dienen, können sie jedoch nicht nach haushaltspolitischen Erfordernissen variiert werden. Die Mehrwertsteuer-Eigenmittel haben sich noch nicht sehr weit von Finanzbeiträgen entfernt: Die Europäischen Gemeinschaften treten gegenüber dem einzelnen Steuerzahler nicht in Erscheinung, sondern haben nur gegen die Mitgliedstaaten einen Anspruch, der nicht an einer tatsächlichen Größe, sondern an der nach der Einnahmenmethode berechneten Mehrwertsteuer-Bemessungsgrundlage anknüpft. Die Bruttosozialprodukt-Eigenmittel besitzen noch offensichtlicher den Charakter von Finanzbeiträgen. Sie dienen außerdem nur der Restfinanzierung.

Die begrenzte Einnahmenautonomie der Europäischen Gemeinschaften hat zu erheblichen Schwierigkeiten geführt. Die Europäischen Gemeinschaften waren in manchen Jahren nicht in der Lage, ihre Ausgaben zu finanzieren; immer wieder gerieten sie in Finanzkrisen. Dies kann jedoch nicht losgelöst vom Problem der Ausgabensteigerungen - vor allem bei den Agrarausgaben - gesehen werden. In den Europäischen Gemeinschaften ist keine Verantwortungseinheit zwischen der Belastung der Bürger mit Abgaben und der Bestimmung der Ausgaben vorhanden. Keines der Gemeinschaftsorgane muß direkt vor den Bürgern höhere Steuern für höhere Ausgaben rechtfertigen. Da es keine direkt am Bürger als Steuerschuldner anknüpfende Gemeinschaftsteuer gibt, sondern die Mitgliedstaaten Schuldner der bedeutendsten Eigenmittel sind, wissen die Bürger in der Regel nicht, wie stark sie durch die Europäischen Gemeinschaften belastet werden. Entsprechende Rückkopplungseffekte einer Gemeinschaftsteuer wären auch nur dann gesichert, wenn die Haushaltsbefugnisse auf das Parlament übergingen.

Die nationalen Einnahmenkompetenzen werden von zwei Seiten durch die Europäischen Gemeinschaften angegriffen. Zum einen wird die nationale $\mathrm{Ob}$ jekthoheit, d. h. das Recht der Steuerfindung und der Ausgestaltung von Steuern, durch die Maßnahmen zur Steuerharmonisierung eingeschränkt. Zum anderen ergeben sich Eingriffe in die nationale Ertragshoheit, da die nationalen Ebenen den Europäischen Gemeinschaften Finanzmittel überlassen müssen. Dabei mußten die Zölle als Einnahmequelle an die Europäischen Gemeinschaften abgetreten werden. Bei den Mehrwertsteuer-Eigenmitteln wird häufig von 
einer geteilten Ertragshoheit gesprochen. Es besteht jedoch keine Verpflichtung für das in der Bundesrepublik Deutschland angewandte Verfahren, die Mehrwertsteuer-Eigenmittel aus den nationalen Mehrwertsteuer-Einnahmen zu finanzieren.

Auf die reduzierte Ertragskompetenz kann durch Ausgabenkürzungen oder Steuererhöhungen reagiert werden. Eine Reduktion nationaler Ausgaben ist dann sinnvoll, wenn die Europäischen Gemeinschaften entsprechende Aufgaben übernommen haben. Wird durch die Aufgabenverlagerung nicht nur die zentrale Ebene in den Mitgliedstaaten entlastet, sollte ein Teil der Anpassungslast an den Finanzbedarf der Europäischen Gemeinschaften auch von den dezentralen Gebietskörperschaften getragen werden. In der Bundesrepublik Deutschland ist allerdings nur der Bund durch die Finanzierung der Europäischen Gemeinschaften belastet, obwohl die Länder ebenfalls von den Ausgaben der Europäischen Gemeinschaften profitieren.

Durch die eingeschränkte Ertragshoheit und Objekthoheit der nationalen Ebenen verringern sich deren Möglichkeiten, Einnahmen für nichtfiskalische Ziele einzusetzen. Werden entsprechende Funktionen nicht durch die Gemeinschaft übernommen, so können die nationalen Ebenen mit den ihnen verbleibenden Mitteln nicht mehr alle Aufgaben lösen.

Die Diskussion um einen angemessenen Finanzierungsanteil der Mitgliedstaaten ist nur möglich, weil Mehrwertsteuer-Eigenmittel und BruttosozialproduktEigenmittel im Grunde den Charakter von Finanzbeiträgen haben. In der britischen Forderung nach "juste retour" und dem aus ihr hervorgehenden Korrekturmechanismus finden sich vor allem äquivalenztheoretische Überlegungen wieder. Allerdings wird hier keine exakte Ermittlung des Nutzens der Mitgliedschaft versucht, sondern es werden lediglich die zurechenbaren Rückflüsse betrachtet. Damit wird jedoch nur ein geringer Teil der ökonomischen Integrationseffekte erfaßt. Auch unter dem Aspekt einer Verteilung der Lasten nach der Wirtschaftskraft liegt dem Korrekturmechanismus für Großbritannien keine konsistente Konzeption zugrunde. Er stellt vielmehr eine Sonderregelung dar, die das Einnahmensystem der Gemeinschaften sehr kompliziert.

Durch die Kappung der Mehrwertsteuer-Bemessungsgrundlage und die Einführung der Bruttosozialprodukt-Eigenmittel sollte das Einnahmensystem der Europäischen Gemeinschaften stärker am Prinzip der Lastenverteilung entsprechend der Wirtschaftskraft orientiert werden, wobei das Sozialprodukt als 
Indikator dient. Die Kappung führte jedoch nur zu einer weiteren Komplizierung des Einnahmensystems, ohne daß diese Zielsetzung durchgehend erreicht wurde. Daß es nicht sinnvoll ist, das gesamte Einnahmensystem der Europäischen Gemeinschaften anhand von Sozialproduktsgrößen zu beurteilen, zeigt auch die Diskussion um die "Regressivität" der Mehrwertsteuer-Eigenmittel. Wenn man lediglich dieses Kriterium in den Vordergrund der Betrachtung stellt, wäre es einfacher und transparenter, wieder zu Finanzbeiträgen entsprechend dem Bruttosozialprodukt zurückzukehren.

In den letzten Jahren ist die Forderung nach einem Ausgleich zwischen den Mitgliedstaaten aus verschiedenen Gründen immer lauter geworden. Da die Ausgleichsmöglichkeiten auf der Einnahmenseite begrenzt waren, versuchte die Kommission auf der Ausgabenseite mehr Gewicht auf die Umverteilung zu legen: Dabei wurde das Umverteilungsziel in Aufgaben hineingetragen, die primär nicht dieser Zielsetzung dienten, wie z. B. die Technologiepolitik und die Strukturfonds. Dies muß auch vor dem Hintergrund gesehen werden, daß die Verteilungswirkungen der Agrarmarktpolitik als unerwünscht angesehen werden und die Strukturfonds hier einen Ausgleich schaffen sollen. Da die Agrarmarktausgaben sehr viel höher als die Ausgaben der Strukturfonds sind, besteht die Gefahr, daß die Strukturfondsmittel mit diesem Argument immer weiter aufgebläht werden. Dies hat jedoch mit der ursprünglichen Zielsetzung der Strukturfonds nichts mehr zu tun hat. Hier muß vielmehr am Agrarfonds selbst angesetzt werden. Von entscheidender Bedeutung wird es daher sein, ob und in welchem Umfang die Europäischen Gemeinschaften die Reform des Agrarfonds vorantreiben. 


\section{Literaturverzeichnis}

Agrarbericht 1989, Agrar- und ernährungspolitischer Bericht der Bundesregierung, in: Bundestagsdrucksache 11/3968. (Zitierweise: = Agrarbericht $1989=)$

Agrarbericht 1991, Agrar- und ernährungspolitischer Bericht der Bundesregierung, in: Bundestagsdrucksache 12/70. (Zitierweise: = Agrarbericht $1991=)$

Akte über die Bedingungen des Beitritts des Königreichs Spanien und der Portugiesischen Republik und die Anpassung der Verträge, in: Amtsblatt der EG, Nr. L 302, vom 15.11.1985.

Andel, Norbert: Europäische Gemeinschaften, in: Handbuch der Finanzwissenschaft, Band IV, hrsg. von Fritz Neumark unter Mitwirkung von Norbert Andel und Heinz Haller, 3., gänzl. neubearbeitete Auflage, Tübingen 1983, S. 311 ff. (Zitierweise: =Europäische Gemeinschaften=)

Arbeitsgruppe "Europäische Verfassung" der Bertelsmann Stiftung: Wie Europa verfaßt sein soll. Vorschläge zur institutionellen Weiterentwicklung der Europäischen Gemeinschaft, Gütersloh 1990. (Zitierweise: =Institutionelle Weiterentwicklung =)

Augstein, Reinhard: Grundzüge der Finanzwirtschaft der Europäischen Gemeinschaft, in: Offentliche Finanzwirtschaft, hrsg. von Volker Arnold und Otto Erich Geske, München 1988, S. 315ff. (Zitierweise: = Finanzwirtschaft der $\mathrm{EG}=$ )

Barre, Bondewjin: EC Tax Scene, Step-by Step, in: International Tax Review, Jg. 1991, S. 282ff. (Zitierweise: =EC Tax Scene=)

Bericht der Bundesregierung über die Entwicklung der Finanzhilfen des Bundes und der Steuervergunstigungen für die Jahre 1981 bis 1984, in: Bundestagsdrucksache 10/352. (Zitierweise: $=9$. Subventionsbericht $=$ )

Bericht der Bundesregierung über die Entwicklung der Finanzhilfen des Bundes und der Steuervergunstigungen für die Jahre 1983 bis 1986, in: Bundestagsdrucksache 10/3821. (Zitierweise: $=10$. Subventionsbericht $=$ )

Bericht der Bundesregierung über die Entwicklung der Finanzhilfen des Bundes und der Steuervergunstigungen für die Jahre 1985 bis 1988, in: Bundestagsdrucksache 11/P338. (Zitierweise: $=11$. Subventionsbericht $=$ )

Bericht der Bundesregierung über die Entwicklung der Finanzhilfen des Bundes und Steuervergünstigungen für die Jahre 1987 bis 1990, in: Bundestagsdrucksache 11/5116. (Zitierweise: $=12$. Subventionsbericht $=$ )

Beschluß über die Einführung allgemeiner unmittelbarer Wahlen der Abgeordneten der Versammlung, abgedruckt in: Europa-Recht, 9., neubearbeitete und ergänzte Auflage, München 1989, S. $235 \mathrm{ff}$. 
Beutler, Bengt; Bieber, Roland; Pipkorn, Jörn; Streit, Jochen: Die Europäische Gemeinschaft. Rechtsordnung und Politik, 3., neubearbeitete Auflage, Baden-Baden 1987. (Zitierweise: =Europäische Gemeinschaft=)

Bickel, Wilhelm: Der Finanzausgleich, in: Handbuch der Finanzwissenschaft, Band II, hrsg. von Wilhelm Gerloff und Fritz Neumark, 2. Auflage, Tübingen 1956, S. 730ff. (Zitierweise: =Finanzausgleich=)

Bieber, Roland: Die Erschließung neuer Finanzierungsmittel, in: Entwicklungsperspektiven der Europäischen Gemeinschaft, hrsg. von Siegfried Magiera, Berlin 1985, S. 37ff. (Zitierweise: = Neue Finanzierungsmittel =)

Biehl, Dieter: Zur Rolle der öffentlichen Finanzen in der Europäischen Integration, in: Integration, 1. Jg. (1978), S. 35ff.

Biehl, Dieter: Finanzausgleich IV: Internationaler Finanzausgleich, in: Handwörterbuch der Wirtschaftswissenschaften, Band 2, hrsg. von Willi Albers et al., Tübingen 1980, S. 689ff. (Zitierweise: =Finanzausgleich IV=)

Biehl, Dieter: Europäische Regionalpolitik. Eine ziel- und handlungsorientierte Analyse, in: Probleme des Finanzausgleichs III, Finanzausgleich im Rahmen der Europäischen Gemeinschaften, hrsg. von Dieter Pohmer, Schriften des Vereins für Socialpolitik, N. F., Band 96/III, Berlin 1981, S. 125ff. (Zitierweise: = Europäische Regionalpolitik =)

Biehl, Dieter: Die Reform der EG-Finanzverfassung aus der Sicht einer ökonomischen Theorie des Föderalismus, in: Wirtschaftspolitik zwischen ökonomischer und politischer Rationalität, Festschrift für Herbert Giersch, hrsg. von Manfred E. Streit, Wiesbaden 1988, S. 63ff. (Zitierweise: =EGFinanzverfassung =)

Biehl, Dieter; Winter, Horst, unter Mitarbeit von Doris Bohlender und Jürgen Kaiser: Die EG-Finanzierung aus föderalistischer Perspektive, in: Europa finanzieren - ein föderalistisches Modell, hrsg. von der Bertelsmann Stiftung, Gütersloh 1990, S. $21 \mathrm{ff}$. (Zitierweise: =EG-Finanzierung=)

Birke, Hans Eberhard: Die deutschen Bundesländer in den Europäischen Gemeinschaften, Berlin 1973.

Biskup, Eckhardt: Die vierte Ebene, in: Die Finanzbeziehungen zwischen Bund, Ländern und Gemeinden aus finanzverfassungsrechtlicher und finanzwirtschaftlicher Sicht, hrsg. vom Bundesministerium der Finanzen, Bonn 1982, S. 581ff. (Zitierweise: =Die vierte Ebene =)

Blaschke, Dieter: Gemeinschaftsaufgabe und EG-Politik, in: Politikverflechtung II, Kritik und Berichte aus der Praxis, hrsg. von Fritz W. Scharpf, B. Reissert, Fritz Schnabel, Kronberg/Ts. 1977, S. 44ff. (Zitierweise: =Gemeinschaftsaufgabe und EG-Politik=)

Blumenwitz, Dieter: Europäische Gemeinschaft und Rechte der Länder, in: Das Europa der Zweiten Generation, Gedächtnisschrift für Christoph Sasse, hrsg. von Roland Bieber et al., Band I, Baden-Baden 1981, S. 215ff. (Zitierweise: =EG und Rechte der Länder=) 
Bock, Sibylle: Instrumente des Finanzausgleichs im Dienste der regionalen Einkommensangleichung in der EG, Frankfurt a. M. 1985. (Zitierweise: $=$ Instrumente des Finanzausgleichs $=$ )

Borchmann, Michael: Verbindungsbüros der Bundesländer bei der EG. Berechtigte Interessenvertretung oder Nebenaußenpolitik? in: Neue Zeitschrift für Verwaltungsrecht, 7. Jg. (1988), S. 218ff. (Zitierweise: $=$ Verbindungsbüros $=$ )

Borell, Rolf: Mischfinanzierungen. Darstellung, Kritik, Reformüberlegungen, hrsg. vom Karl-Bräuer-Institut des Bundes der Steuerzahler e. V., Wiesbaden 1981. (Zitierweise: = Mischfinanzierungen=)

Breton, Albert: Öffentliche Güter und die Stabilität des Föderalismus, in: Föderalismus, hrsg. von Guy Kirsch, Stuttgart, New York 1977, S. $128 \mathrm{ff}$.

Brümmerhoff, Dieter: Finanzwissenschaft, 5., überarbeitete und erweiterte Auflage, München, Wien 1990. (Zitierweise: =Finanzwissenschaft=)

Buchanan, James N.: Wer sollte was in einem föderativen System verteilen, in: Föderalismus, hrsg. von Guy Kirsch, Stuttgart, New York 1977, S. 51 ff. (Zitierweise: $=$ Föderatives System $=$ )

Buchanan, James N.; Tullock, Gordon: The Calculus of Consent. Logical Foundations of Constitutional Democracy, Ann Arbor 1962. (Zitierweise: $=$ Calculus of Consent $=$ )

Bundesministerium der Finanzen: Finanzbericht 1972. Die volkswirtschaftlichen Grundlagen und die wichtigsten finanzwirtschaftlichen Probleme des Bundeshaushaltsplans für das Haushaltsjahr 1972, Bonn 1971. (Zitierweise: = Finanzbericht $1972=$ )

Bundesministerium der Finanzen: Finanzbericht 1973. Die volkswirtschaftlichen Grundlagen und die wichtigsten finanzwirtschaftlichen Probleme des Bundeshaushaltsplans für das Haushaltsjahr 1973, Bonn 1973. (Zitierweise: =Finanzbericht $1973=$ )

Bundesministerium der Finanzen: Finanzbericht 1974. Die volkswirtschaftlichen Grundlagen und die wichtigsten finanzwirtschaftlichen Probleme des Bundeshaushaltsplans für das Haushaltsjahr 1974, Bonn 1973. (Zitierweise: =Finanzbericht $1974=$ )

Bundesministerium der Finanzen: Finanzbericht 1975. Die volkswirtschaftlichen Grundlagen und die wichtigsten finanzwirtschaftlichen Probleme des Bundeshaushaltsplans für das Haushaltsjahr 1975, Bonn 1974. (Zitierweise: =Finanzbericht $1975=$ )

Bundesministerium der Finanzen: Finanzbericht 1976. Die volkswirtschaftlichen Grundlagen und die wichtigsten finanzwirtschaftlichen Probleme des Bundeshaushaltsplans für das Haushaltsjahr 1976, Bonn 1975. (Zitierweise: =Finanzbericht 1976=)

Bundesministerium der Finanzen: Finanzbericht 1977. Die volkswirtschaftlichen Grundlagen und die wichtigsten finanzwirtschaftlichen Probleme des Bundeshaushaltsplans für das Haushaltsjahr 1977, Bonn 1977. (Zitierweise: = Finanzbericht $1977=$ ) 
Bundesministerium der Finanzen: Finanzbericht 1978. Die volkswirtschaftlichen Grundlagen und die wichtigsten finanzwirtschaftlichen Probleme des Bundeshaushaltsplans für das Haushaltsjahr 1978, Bonn 1977. (Zitierweise: =Finanzbericht $1978=$ )

Bundesministerium der Finanzen: Finanzbericht 1979. Die volkswirtschaftlichen Grundlagen und die wichtigsten finanzwirtschaftlichen Probleme des Bundeshaushaltsplans für das Haushaltsjahr 1979, Bonn 1978. (Zitierweise: =Finanzbericht $1979=$ )

Bundesministerium der Finanzen: Finanzbericht 1980. Die volkswirtschaftlichen Grundlagen und die wichtigsten finanzwirtschaftlichen Probleme des Bundeshaushaltsplans für das Haushaltsjahr 1980, Bonn 1979. (Zitierweise: =Finanzbericht $1980=$ )

Bundesministerium der Finanzen: Finanzbericht 1981. Die volkswirtschaftlichen Grundlagen und die wichtigsten finanzwirtschaftlichen Probleme des Bundeshaushaltsplans für das Haushaltsjahr 1981, Bonn 1980. (Zitierweise: =Finanzbericht $1981=$ )

Bundesministerium der Finanzen: Finanzbericht 1982. Die volkswirtschaftlichen Grundlagen und die wichtigsten finanzwirtschaftlichen Probleme des Bundeshaushaltsplans für das Haushaltsjahr 1982, Bonn 1981. (Zitierweise: =Finanzbericht $1982=$ )

Bundesministerium der Finanzen: Finanzbericht 1983. Die volkswirtschaftlichen Grundlagen und die wichtigsten finanzwirtschaftlichen Probleme des Bundeshaushaltsplans für das Haushaltsjahr 1983, Bonn 1982. (Zitierweise: =Finanzbericht $1983=$ )

Bundesministerium der Finanzen: Finanzbericht 1984. Die volkswirtschaftlichen Grundlagen und die wichtigsten finanzwirtschaftlichen Probleme des Bundeshaushaltsplans für das Haushaltsjahr 1984, Bonn 1983. (Zitierweise: =Finanzbericht $1984=$ )

Bundesministerium der Finanzen: Finanzbericht 1985. Die volkswirtschaftlichen Grundlagen und die wichtigsten finanzwirtschaftlichen Probleme des Bundeshaushaltsplans für das Haushaltsjahr 1985, Bonn 1984. (Zitierweise: =Finanzbericht $1985=$ )

Bundesministerium der Finanzen: Finanzbericht 1986. Die volkswirtschaftlichen Grundlagen und die wichtigsten finanzwirtschaftlichen Probleme des Bundeshaushaltsplans für das Haushaltsjahr 1986, Bonn 1985. (Zitierweise: =Finanzbericht $1986=$ )

Bundesministerium der Finanzen: Der Haushalt der Europäischen Gemeinschaft 1986. Zahlen, Daten, Fakten, in: BMF-Dokumentation, Nr. 7/1986, Bonn 1986. (Zitierweise: =EG-Haushalt 1986=)

Bundesministerium der Finanzen: Finanzbericht 1987. Die volkswirtschaftlichen Grundlagen und die wichtigsten finanzwirtschaftlichen Probleme des Bundeshaushaltsplans für das Haushaltsjahr 1987, Bonn 1986. (Zitierweise: =Finanzbericht $1987=$ ) 
Bundesministerium der Finanzen: Finanzbericht 1988. Die volkswirtschaftlichen Grundlagen und die wichtigsten finanzwirtschaftlichen Probleme des Bundeshaushaltsplans für das Haushaltsjahr 1988, Bonn 1987. (Zitierweise: =Finanzbericht $1988=$ )

Bundesministerium der Finanzen: Finanzbericht 1989. Die volkswirtschaftlichen Grundlagen und die wichtigsten finanzwirtschaftlichen Probleme des Bundeshaushaltsplans für das Haushaltsjahr 1989, Bonn 1988. (Zitierweise: =Finanzbericht $1989=$ )

Bundesministerium der Finanzen: Der Haushalt der Europäischen Gemeinschaft 1989. Zahlen und Tabellen, in: BMF-Dokumentation, Nr. 3/1989, Bonn 1989. (Zitierweise: =EG-Haushalt 1989=)

Bundesministerium der Finanzen: Der Haushalt der Europäischen Gemeinschaft 1990. Zahlen und Tabellen, in: BMF-Dokumentation, Nr. 5/1990, Bonn 1990. (Zitierweise: =EG-Haushalt 1990=)

Bundesministerium der Finanzen: Finanzbericht 1990. Die volkswirtschaftlichen Grundlagen und die wichtigsten finanzwirtschaftlichen Probleme des Bundeshaushaltsplans für das Haushaltsjahr 1990, Bonn 1989. (Zitierweise: =Finanzbericht $1990=$ )

Bundesministerium der Finanzen: Finanzbericht 1991. Die volkswirtschaftlichen Grundlagen und die wichtigsten finanzwirtschaftlichen Probleme des Bundeshaushaltsplans für das Haushaltsjahr 1991, Bonn 1991. (Zitierweise: =Finanzbericht $1991=$ )

Bundesministerium der Finanzen: Finanzbericht 1992. Die volkswirtschaftlichen Grundlagen und die wichtigsten finanzwirtschaftlichen Probleme des Bundeshaushaltsplans für das Haushaltsjahr 1992, Bonn 1991. (Zitierweise: =Finanzbericht $1992=$ )

Bundesministerium für Wirtschaft: Regionale Wirtschaftsförderung in der Bundesrepublik Deutschland, Bonn 1988. (Zitierweise: = Regionalpolitik=)

Bungarten, Harald H.: Umweltpolitik in Westeuropa, Bonn 1978. (Zitierweise: =Umweltpolitik =)

Caesar, Rolf: Neue Steuern für die Europäische Gemeinschaft? - Kriterien und Vorschläge, in: Zur Reform der EG-Finanzverfassung, hrsg. von Dieter Biehl und Gero Pfennig, Bonn 1990, S. 57ff. (Zitierweise: =Neue Steuern für die $\mathrm{EG}=$ )

Cecchini, Paolo, unter Zusammenarbeit mit Michel Catinat und Alexis Jacquemin: Europa '92. Der Vorteil des Binnenmarktes. Baden-Baden 1988. (Zitierweise: =Europa '92=)

Delors, Jacques: Arbeitsdokument des Präsidenten der EG-Kommission für das Treffen mit den Ministerpräsidenten der Bundesländer am 19. Mai 1988 in Bonn, in: Europa-Archiv, Folge 1 (Dokumente), 43. Jg. (1988), S. $337 \mathrm{ff}$. (Zitierweise: $=$ Treffen mit den Ministerpräsidenten=) 
Deutsche Bundesbank: An- und Verkaufskurse der Deutschen Bundesbank im Rahmen der Europäischen Währungssystems (EWS), in: Auszüge aus Presseartikeln, hrsg. von der Deutschen Bundesbank, Nr. 79 vom 9.10.1990, S. 1. (Zitierweise: = An- und Verkaufskurse im Rahmen des EWS =)

Deutsche Bundesbank: Stellungnahme der Deutschen Bundesbank zur Errichtung einer Wirtschafts- und Währungsunion in Europa, in: Monatsberichte der Deutschen Bundesbank, 42. Jg. (1990), S. 41 ff. (Zitierweise: = Errichtung einer WWU=)

Deutscher Städtetag: Der Europäische Binnenmarkt und die Städte, in: Reihe K, DST-Beiträge zur städtischen Europaarbeit, Heft 1, 1989. (Zitierweise: $=$ Binnenmarkt und Städte $=$ )

Dichtl, Erwin: Schritte zum Europäischen Binnenmarkt, in: Wirtschaftswissenschaftliches Studium (WiSt), 18. Jg. (1989), S. 333ff. (Zitierweise: $=$ Europäischer Binnenmarkt=)

Dierkes, Meinolf; Hartje, Volkmar: Internationale Aspekte der Umweltpolitik, in: Handbuch fur Planung, Gestaltung und Schutz der Umwelt, hrsg. von K. Buchwald und W. Engelhardt, München, Wien, Zürich 1980, S. 92ff. (Zitierweise: =Internationale Aspekte=)

Dürig, Günter: Kommentar zum Grundgesetz, Art. 1, in: Maunz-Dürig, Band I, Art. 1-15, von Theodor Maunz, Günter Dürig u. a., München' 1983, Stand: 28. Lieferung, Februar 1990. (Zitierweise: $=$ Kommentar $=$ )

Ebers, Mark: Die Europäischen Gemeinschaften. Ihre institutionelle Struktur, Verwaltungsorganisation, Verwaltungsverfahren und Finanzen, in: Wirtschaftswissenschaftliches Studium (WiSt), 18. Jg. (1989), S. 357ff. (Zitierweise: =Institutionelle Struktur =)

Eickhof, Norbert; Berkelova, Marie: Verkehrspolitik in der Bundesrepublik und der EG, in: Wirtschaftswissenschaftliches Studium (WiSt), 19. Jg. (1990), S. 594ff. (Zitierweise: = Verkehrspolitik=)

Einheitliche Europäische Akte, abgedruckt in: Europa-Archiv, Folge 1 (Dokumente), 41. Jg. (1986), S. $163 \mathrm{ff}$.

Endres, Alfred: Die Coase Kontroverse, in: Zeitschrift für die gesamte Staatswissenschaft, Bd. 133 (1977), S. $637 \mathrm{ff}$. (Zitierweise: =Coase-Kontrover$\mathrm{se}=$ )

Ergänzung der Geschäftsordnung des Bundesrates, in: Bundesratsdrucksache Nr. 230/88. (Zitierweise: = Ergänzung der Geschäftsordnung des Bundesrates $=$ )

Europa-Kommission des deutschen Bundestages: Elfter Bericht und Empfehlung, in: Bundestagsdrucksache 10/5271. (Zitierweise: =Elfter Bericht $=$ )

Europäischer Rat: Tagung der Staats- und Regierungschefs der EG am 11. und 12. Februar 1988. Schlußfolgerungen des Europäischen Rates, in: Bulletin, hrsg. vom Presse- und Informationsamt der Bundesregierung, Nr. 27 vom 24.2.1988, S. 225ff. (Zitierweise: = Schlußfolgerungen 1988=) 
Europäischer Rat: Tagung der Staats- und Regierungschefs der EG am 26. und 27. Juni 1989. Schlußfolgerungen des Vorsitzes, in: Bulletin hrsg. vom Presse- und Informationsamt der Bundesregierung, Nr. $69 \mathrm{vom}$ 30.6.1989, S. 605ff. (Zitierweise: =Schlußfolgerungen 1989=)

Europäischer Rat: Sondertagung der Staats- und Regierungschefs der EG am 27. und 28. Oktober 1990. Schlußfolgerungen des Vorsitzes, in: Bulletin, hrsg. vom Presse- und Informationsamt der Bundesregierung, Nr. 128 vom 6.11.1990, S. 1333ff. (Zitierweise: =Schlußfolgerungen 1990=)

Europäischer Rechnungshof: Jahresbericht zum Haushaltsjahr 1985, in: Amtsblatt der EG, Nr. C 321 vom 15.12.1986. (Zitierweise: =Jahresbericht $1985=$ )

Europäischer Rechnungshof: Jahresbericht zum Haushaltsjahr 1987, in: Amtsblatt der EG, Nr. C 316 vom 12.12.1988. (Zitierweise: =Jahresbericht $1987=$ )

Europäischer Rechnungshof: Jahresbericht zum Haushaltsjahr 1988, in: Amtsblatt der EG, Nr. C 312 vom 12.12.1989. (Zitierweise: =Jahresbericht $1988=$ )

Europäischer Rechnungshof: Jahresbericht zum Haushaltsjahr 1989, in: Amtsblatt der EG, Nr. C 313 vom 12.12.1990. (Zitierweise: =Jahresbericht $1989=$ )

Europäisches Parlament: Endgültige Feststellung des Gesamthaushaltsplans der Europäischen Gemeinschaft für das Haushaltsjahr 1989, in: Amtsblatt der EG, Nr. L 26 vom 30.1.1989. (Zitierweise: =Haushaltsplan 1989=)

Europäisches Parlament: Endgültige Feststellung des Gesamthaushaltsplans der Europäischen Gemeinschaft für das Haushaltsjahr 1990, in: Amtsblatt der EG, Nr. L 24 vom 29.1.1990. (Zitierweise: = Haushaltsplan 1990=)

Europäisches Parlament: Die Auswirkungen der Vereinigung Deutschlands auf die Europäische Gemeinschaft, in: Sammlung Wissenschaft und Dokumentation, Luxemburg 1990. (Zitierweise: =Vereinigung Deutschlands=)

Europäisches Parlament: Endgültige Feststellung des Berichtigungs- und Nachtragshaushaltsplans Nr. 2 der Europäischen Gemeinschaften für das Haushaltsjahr 1990, in: Amtsblatt der EG, Nr. L 239 vom 3.9.1990.

Europäisches Parlament: Endgültige Feststellung des Gesamthaushaltsplans der Europäischen Gemeinschaft für das Haushaltsjahr 1991, in: Amtsblatt der EG, Nr. L 30, vom 4.2.1991. (Zitierweise: = Haushaltsplan 1991=)

Europäisches Parlament: Endgültige Feststellung des Gesamthaushaltsplans der Europäischen Gemeinschaft für das Haushaltsjahr 1992, in: Amtsblatt der EG, Nr. L 30, vom 3.2.1992. (Zitierweise: = Haushaltsplan 1992=)

Faber, Malte; Breyer, Friedrich: Eine ökonomische Analyse konstitutioneller Aspekte der europăiischen Integration, in: Jahrbuch für Sozialwissenschaft, Band 31 (1980), S. 213ff. (Zitierweise: =Konstitutionelle Aspekte=) 
Finanzen der Europäischen Gemeinschaften 1984 bis 1988, in: Wirtschaft und Statistik, Jg. 1988, S. 799ff. (Zitierweise: =Finanzen der EG=)

Fischer, Hans-Lothar: Fluch des Kollektivs, in: Wirtschaftswoche, 43. Jg. (1989), S. 84ff. (Zitierweise: = Fluch des Kollektivs=)

Fischer, Helmut: Ein EG-Zuschlag zur Einkommensteuer - eine kritische Stellungnahme, in: Europa finanzieren. Ein föderalistisches Modell, hrsg. von der Bertelsmann Stiftung, Gütersloh 1990, S. 133ff. (Zitierweise: $=$ EGZuschlag =)

Fischer-Menshausen, Herbert: Finanzausgleich II. Grundzüge des Finanzausgleichsrechts, in: Handwörterbuch der Wirtschaftswissenschaften, hrsg. von Willi Albers et al., Band 2, Tübingen 1980, S. 636ff. (Zitierweise: $=$ Finanzausgleich $\mathrm{II}=$ )

Fleckenstein, Kurt: Aufgabenteilung in der regionalen Wirtschaftsförderung, in: Raumforschung und Raumordnung, 43. Jg. (1985), S. 173ff. (Zitierweise: $=$ Regionale Wirtschaftsförderung $=$ )

Forte, Francesco: Grundsätze der Zuordnung öffentlicher ökonomischer Funktionen im Rahmen von Gebietskörperschaften, in: Bericht der Sachverständigengruppe zur Untersuchung der Rolle der öffentlichen Finanzen bei der Europäischen Integration, Band II, hrsg. von der Kommission der EG, Brüssel 1977, S. 335ff. (Zitierweise: = Zuordnung ökonomischer Funktionen =)

Franke, Jürgen: Die Regionalpolitik der Europäischen Gemeinschaft. Eine theoretische und empirische Analyse ihres Wirkungsgrades und der Entwurf eines Systems konkurrierender Regionen als Ergänzung zur Strategie der Marktintegration in der Gemeinschaft, Bochum 1989. (Zitierweise: $=$ Regionalpolitik der $\mathrm{EG}=$ )

Franzmeyer, Fritz; Seidel, Bernhard: Supranationaler Finanzausgleich und europäische Integration, Bonn 1976. (Zitierweise: =Uberstaatlicher Finanzausgleich $=$ )

Frey, Dieter: Die Finanzverfassung des Grundgesetzes, in: Die Finanzbeziehungen zwischen Bund, Ländern und Gemeinden aus finanzverfassungsrechtlicher und finanzwirtschaftlicher Sicht, hrsg. vom Bundesministerium der Finanzen, Bonn 1982, S. 13ff. (Zitierweise: = Finanzverfassung=)

Frey, René L.: Zwischen Föderalismus und Zentralismus. Ein volkswirtschaftliches Konzept des schweizerischen Bundesstaates, Bern, Frankfurt a. M. 1977. (Zitierweise: = Föderalismus und Zentralismus=)

Füchsel, Wolf-Dieter: Gemeinschaftsaufgaben, Bamberg 1985. (Zitierweise: $=$ Gemeinschaftsaufgaben $=$ )

Gesetz zur Einheitlichen Europäischen Akte vom 19.12.1986, in: Bundesgesetzblatt, Jg. 1986, Teil Il, S. 1102f. (Zitierweise: =Gesetz zur Einheitlichen Europaischen Akte =) 
Gesetzantrag der Länder Bayern, Hessen, Nordrhein-Westfalen und Rheinland-Pfalz. Entwurf eines ... Gesetzes zur Anderung des Grundgesetzes (Artikel 24 Abs. 166), in: Bundesratsdrucksache 703/89. (Zitierweise: $=$ Gesetzantrag der Länder $=$ )

Grabitz, Eberhard: Die Rechtsetzungsbefugnis von Bund und Ländern bei der Durchführung von Gemeinschaftsrecht, in: Archiv des öffentlichen Rechts, Band 111 (1986), S. 1ff. (Zitierweise: = Rechtsetzungsbefugnis=)

Grossekettler, Heinz: Zentralisation und Dezentralisation der Wirtschaftsförderung aus wirtschaftswissenschaftlicher Sicht, in: Kommunale Wirtschaftsförderung, hrsg. von D. Ehlers, Köln u. a. 1990, S. $71 \mathrm{ff}$.

Haas, Evelyn: Die Mitwirkung der Länder bei EG-Vorhaben. Neuere Entwicklungen im Gefolge der Luxemburger Akte, in: Die öffentliche Verwaltung, 41. Jg. (1988), S. 613ff. (Zitierweise: = Mitwirkung der Län$\operatorname{der}=$ )

Hackenbroch, Inge: Zur Theorie und Politik eines supranationalen Finanzausgleichs. Gestaltungsprobleme und Gestaltungsansätze unter besonderer Berücksichtigung der EG, München, Köln, London 1983. (Zitierweise: =Supranationaler Finanzausgleich $=$ )

Haller, Heinz: Die Steuern. Grundlinien eines rationalen Systems öffentlicher Abgaben, 3., überarbeitete Auflage, Tübingen 1981. (Zitierweise: =Steuern =)

Handbuch des Europäischen Rechts, hrsg. von Claus-Dieter Ehlermann und Roland Bieber, Stand 69. Lieferung, Januar 1991.

Hansmeyer, Karl-Heinrich: Zweckzuweisungen an Gemeinden als Mittel der Wirtschaftspolitik, in: Theorie und Praxis des finanzpolitischen Interventionismus, hrsg. von Heinz Haller et al., Tübingen 1970, S. $431 \mathrm{ff}$. (Zitierweise: =Zweckzuweisungen $=$ )

Hansmeyer, Karl-Heinrich; Kops, Manfred: Die Kompetenzarten der Aufgabenzuständigkeit und deren Verteilung im föderalistischen Staat, in: Hamburger Jahrbücher für Wirtschafts- und Gesellschaftspolitik, 29. Jg. (1984), S. 127ff. (Zitierweise: = Aufgabenzuständigkeit=)

Hansmeyer, Karl-Heinrich; Kops, Manfred: Interdependenzen im passiven Finanzausgleich, in: Offentliche Finanzen, Kredit und Kapital, hrsg. von Dieter Cansier und Dietmar Kath, Berlin 1985, S. 3ff. (Zitierweise: $=$ Passiver Finanzausgleich $=$ )

Hanusch, Horst: Erfüllung von Staatsaufgaben im Föderalismus. Eine ökonomisch-politische Analyse auftretender Konflikte, in: Wirtschaftswissenschaften als Grundlage staatlichen Handelns, hrsg. von Peter Bohley und Georg Tolkemitt, Tübingen 1979, S. 359-381. (Zitierweise: = Staatsaufgaben $=$ )

Harbrecht, Wolfgang: Die Europäische Gemeinschaft, 2., neubearbeitete und erweiterte Auflage, Stuttgart 1984. (Zitierweise: $=\mathbf{E G}=$ )

Hasse, Rolf H.: Zweite Stufe als Mittel zur Erfolgskontrolle, in: Wirtschaftsdienst, 70. Jg. (1990), S. 341 ff. (Zitierweise: =Zweite Stufe =) 
Haushaltsplan des Saarlandes für das Haushaltsjahr 1990.

Hellwig, Renate: Die Rolle der Bundesländer in der Europa-Politik, in: Europa-Archiv, Folge 2 (Beiträge und Berichte), 42. Jg. (1987), S. $297 \mathrm{ff}$. (Zitierweise: = Rolle der Bundesländer=)

Henke, Klaus-Dirk: Europäische Integration als Problem des Finanzausgleichs, in: Wirtschaftsdienst, 60. Jg. (1980), S. $24 \mathrm{ff}$.

Henke, Klaus-Dirk: Die Finanzierung der Europäischen Gemeinschaften. Zur integrationsfördernden Fortentwicklung des europäischen Einnahmensystems, in: Probleme des Finanzausgleichs III, Finanzausgleich im Rahmen der Europäischen Gemeinschaften, hrsg. von Dieter Pohmer, Schriften des Vereins für Socialpolitik, N. F., Band 96/III, Berlin 1981, S. $11 \mathrm{ff}$. (Zitierweise: =Finanzierung der $\mathrm{EG}=$ )

Henke, Klaus-Dirk: Sozialproduktsteuer, in: Wirtschaftswissenschaftliches Studium (WiSt), 17. Jg. (1988), S. 140ff.

Henze, Arno; Zeddies, Jürgen: Eine erste Analyse der Auswirkungen der Beschlüsse zur Reform der Agrarpolitik in der EG, in: Agrarwirtschaft, 37. Jg. (1988), S. $161 \mathrm{ff}$. (Zitierweise: = Reform der Ágrarpolitik=)

Herrmann, Anneliese; Ochel, Wolfgang; Wegner, Manfred: Die Auswirkungen des europäischen Binnenmarktes auf die deutsche Wirtschaft und Wirtschaftspolitik, in: IFO-Schnelldienst, Nr. 5-6/90, S. 14ff. (Zitierweise: $=$ Auswirkungen $=$ )

Herz, Bernhard; Nürk, Bettina: Die erste Phase der Europäischen Wirtschafts- und Währungsunion, in: Wirtschaftsdienst, 70. Jg. (1990), S. 313ff. (Zitierweise: =Erste Phase $=$ )

Hirn, Wolfgang: Großes Vorbild Fed. Die EG-Kommission fodert eine unabhăngige europäische Zentralbank, in: Wirtschaftswoche, Nr. 14 vom 30.3.1990, S. 32f. (Zitierweise: =Großes Vorbild Fed =)

Hirn, Wolfgang: Mafiosi im Schlachthaus. Agrarbetrügereien ohne Grenzen, in: Wirtschaftsdienst, Nr. 41 vom 6.10.1989, \$. 56ff. (Zitierweise: $=$ Agrarbetrügereien $=$ )

Hirn, Wolfgang; Stimpfel, Roland: Stau ohne Grenzen, in: Wirtschaftswoche, Nr. 15 vom 6.4.1990, S. 46ff. (Zitierweise: =Stau ohne Grenzen=)

Hirn, Wolfgang; Thelen, Friedrich: Europäische Gemeinschaft. Das neue Empire setzt seine Normen durch Einheit, Vielfalt, Einfalt, in: Wirtschaftswoche, Nr. 19 vom 4.5.1990, S. 14ff.' (Zitierweise: =Einheit, Vielfalt, Einfalt =)

Holzheu, Franz: Wirtschaftspolitik für Europa, in: Finanzarchiv, N. F., Band 48 (1990), S. $1 \mathrm{ff}$.

Hort, Peter: Europa in neuer Gestalt, in: Frankfurter Allgemeine Zeitung vom 17.12.1990, S. 1. (Zitierweise: =Europa in neuer Gestalt =) 
Hrbek, Rudolf: Politikverflechtung macht an den Grenzen nicht halt. Auswirkungen der EG-Mitgliedschaft auf die föderative Ordnung der Bundesrepublik Deutschland, in: Der Bürger im Staat, 29. Jg. (1979), S. 38ff. (Zitierweise: $=$ Politikverflechtung $=$ )

Hrbek, Rudolf: Doppelte Politikverflechtung: Deutscher Föderalismus und Europäische Integration. Die deutschen Länder im EG-Entscheidungsprozeß, in: Die deutschen Länder und die Europäischen Gemeinschaften, hrsg. von Rudolf Hrbek und Uwe Thaysen, Baden-Baden 1986, S. 17ff. (Zitierweise: =Doppelte Politikverflechtung =)

Isensee, Josef: Subsidiaritätsprinzip und Verfassungsrecht. Eine Studie über das Regulativ des Verhältnisses von Staat und Gesellschaft, Berlin 1968. (Zitierweise: =Subsidiaritätsprinzip und Verfassungsrecht $=$ )

Internes Abkommen über die Finanzierung und Verwaltung der Hilfen der Gemeinschaft im Rahmen des Vierten ARP-EWG-Abkommens, in: Amtsblatt der EG, Nr. L 229 vom 17.8.1991, S. $288 \mathrm{ff}$.

Issing, Ottmar: Perspektiven der zukünftigen Weltwährungsordnung. Evolution versus institutionelle Reform, Referat auf der Tagung des Vereins für Socialpolitik am 5.10.1990 in Würzburg, abgedruckt in: Auszüge aus Presseartikeln, hrsg. von der Deutschen Bundesbank, Nr. 81 vom 16.10.1990, S. $4 \mathrm{ff}$.

Issing, Ottmar: EWS, Währungsunion und Kapitalallokation. Neue Perspektiven durch die deutsche Wiedervereinigung. Vortrag auf einem Symposium des Instituts für Empirische Wirtschaftsforschung am 22.2.1991 in Berlin, abgedruckt in: Auszüge aus Presseartikeln, hrsg. von der Deutschen Bundesbank, Nr. 13 vom 27.3.1991, S. 1ff. (Zitierweise: =EWS=)

Issing, Ottmar: Europäische Währungsintegration. Ausgangslage, Strategien, Risiken, in: Probleme der Vollendung des Binnenmarktes in Europa nach 1992, hrsg. von Erhard Kantzenbach, Schriften des Vereins für Socialpolitik, N. F., Bd. 199, Berlin 1990, S. 111-136. (Zitierweise: =Europäische Währungsintegration =)

Jaeger, Klaus: Steuern, Entschädigungen und das Coase Theorem, in: Jahrbuch für Sozialwissenschaften, Bd. 26 (1975), S. 209ff. (Zitierweise: $=$ Coase Theorem $=$ )

Jarchow, Hans-Joachim: Theorie und Politik des Geldes II. Geldmarkt, Bundesbank und geldpolitisches Instrumentarium, 3., überarbeitete und erweiterte Auflage, Göttingen 1979. (Zitierweise: =Geldmarkt=)

Kaiser, Joseph H.: Regionalpolitik im föderativen System der Bundesrepublik Deutschland und der Europäischen Gemeinschaft, in: Festschrift für Theodor Maunz zum 80. Geburtstag, hrsg. von Peter Lerche, Hans Zacher und Peter Badura, München 1981, S. 169ff. (Zitierweise: = Regionalpolitik=)

Kamp, Ernst M.; Langheinrich, Camilla; Stamm, Friedrich H.: Die Ordnung der öffentlichen Finanzen, zugleich 3 . Auflage des ersten Teiles, Kapitel 1 bis 3 und des zweiten Teiles, Kapitel 1 von "Das optimale Finanzsystem", Bonn 1971. (Zitierweise: =Optimales Finanzsystem=) 
Karl-Bräuer-Institut des Bundes der Steuerzahler: EG-Finanzen, Grundsätzliche Reformen sind unerläßlich, Wiesbaden 1988. (Zitierweise: =EGFinanzen =)

Kessler, Martin: Wildes Dickicht. EG-Industriepolitik: Milliarden ohne Konzept und Kontrolle, in: Wirtschaftswoche Nr. 37 vom 6.9.1991, S. 46ff. (Zitierweise: =Wildes Dickicht $=$ )

Kielmansegg, Peter Graf: Ohne historisches Vorbild. Probleme der Europäischen Gemeinschaft auf dem Weg zur Politischen Union, in: Frankfurter Allgemeine Zeitung vom 7.12.1990, S. 13. (Zitierweise: =Weg zur Politischen Union=)

Kirsch, Guy: Ökonomische Theorie der Politik, Tübingen 1974. (Zitierweise: $=$ Okonomische Theorie $=$ )

Kirsch, Guy: Föderalismus. Die Wahl zwischen intrakollektiver Konsenssuche und interkollektiver Auseinandersetzung, in: Probleme des Finanzausgleichs I, hrsg. von Wilhelmine Dreissig, Schriften des Vereins für Socialpolitik, N. F., Band 96/I, Berlin 1978, S. 9ff. (Zitierweise: = Die Wahl=)

Kirsch, Guy; Theiler, Jörg: Zur Verallgemeinerung von Buchanan-Tullocks allgemeiner ökonomischer Verfassungstheorie, in: Finanzarchiv, N. F., Band 35 (1976/77), S. 35ff. (Zitierweise: =Verallgemeinerung=)

Klemmer, Paul: Harmonisierung der Umweltpolitik in der EG, in: Wirtschaftsdienst, 71. Jg. (1991), S. 262ff. (Zitierweise: = Harmonisierung der Umweltpolitik =)

Kloten, Norbert: Der "Delors-Bericht" in: Europa-Archiv, Folge 2 (Beiträge und Berichte), 44. Jg. (1989), S. $251 \mathrm{ff}$. (Zitierweise: = Delors-Bericht=)

Knödgen, Gabriele; Sprenger, Rolf-Ulrich: Umweltschutz und internationaler Wettbewerb, in: FO-Schnelldienst, Nr. 1-2/81, S. 29ff. (Zitierweise: $=$ Umweltschutz $=$ )

Koester, Ulrich; Terwitte, Heinrich: Durchbruch in der Agrarpolitik oder weiteres Politikversagen, in: Wirtschaftsdienst, 17. Jg. (1988), S. $130 \mathrm{ff}$. (Zitierweise: $=$ Politikversagen $=$ )

Kommission der EG: Bericht der Kommission über die Europäische Union, in: Bulletin der Europäischen Gemeinschaften, Beilage 5/75, Brüssel 1975. (Zitierweise: = Europäische Union=)

Kommission der EG (Hrsg.): Bericht der Sachverständigengruppe zur Untersuchung der Rolle der offentlichen Finanzen bei der Europäischen Integration, Band 1, Brüssel, Luxemburg 1977. (Zitierweise: =McDougall-Bericht $=$ )

Kommission der EG: Finanzierung des Gemeinschaftshaushalts. Künftige Methoden, in: Bulletin der Europässchen Gemeinschaften, Beilage 8/78, Brüssel 1979. (Zitierweise: = Finanzierung des Gemeinschaftshaushalts=) 
Kommission der EG: Mitteilung über staatliche Beihilfen - Bundesrepublik Deutschland (Artikel 92 bis 94 EWG-Vertrag) - Mitteilung gemäß Artikel 93 Absatz 2 erster Satz des EWG-Vertrags an andere Beteiligte als die Mitgliedstaaten über die regionalen Beihilfen der Länder der Bundesrepublik Deutschland, in: Amtsblatt der EG, Nr. C 340 vom 20.12.1984, S. 3. (Zitierweise: = Regionale Beihilfen 1984=)

Kommission der EG: Entscheidung über das regionale Wirtschaftsförderungsprogramm des Landes Nordrhein-Westfalen, in: Amtsblatt der EG, Nr. L T vom 9.1.1985, S. 28ff. (Zitierweise: = Wirtschaftsförderungsprogramm Nordrhein-Westfalen $1985=$ )

Kommission der EG: Entscheidung Nr. 3484/85/EGKS vom 27.11.1985 zur Einführung gemeinschaftlicher Beihilfen zugunsten der Eisen- und Stahlindustrie, in: Amtsblatt der EG, Nr. L 340 vom 18.12 .1985 , S. $1 \mathrm{ff}$. (Zitierweise: =Beihilfen Eisen- und Stahlindustrie 1985=)

Kommission der EG: Vollendung des Binnenmarktes. Weißbuch der Kommission an den Rat, Luxemburg 1985. (Zitierweise: =Vollendung des Binnenmarktes $=$ )

Kommission der EG: Vierter Jahresbericht an das Europäische Parlament über die Kontrolle der Anwendung des Gemeinschaftsrechts - 1986, in: Amtsblatt der EG, Nr. C 338 vom 16.12.1987, S. 1ff. (Zitierweise: =Anwendung des Gemeinschaftsrechts 1986=)

Kommission der EG: Bulletin der Europäischen Gemeinschaften, 20. Jg., Brüssel 1987. (Zitierweise: =Bulletin 1987=)

Kommission der EG: Entscheidung vom 19.2.1986 über die Vereinbarkeit von regionalen Beihilfen mit dem Gemeinsamen Markt in sechs nach der Gemeinschaftsaufgabe "Verbesserung der regionalen Wirtschaftsstruktur" geförderten Arbeitsmarktregionen, in: Amtsblatt der EG, Nr. L 12 vom 14.1.1987, S. 17ff. (Zitierweise: = Regionale Beihilfen 1986=)

Kommission der EG: Europäischer Fonds für regionale Entwicklung, Jahresbericht 1985, Luxemburg 1987. (Zitierweise: =EFRE-Jahresbericht $1985=)$

Kommission der EG: Richtlinie vom 7.4.1987 zur Anpassung der Richtlinie 84/538/EWG des Rates zur Angleichung der Rechtsvorschriften der Mitgliedstaaten über den zulässigen Schalleistungspegel von Rasenmähern, in: Amtsblatt der EG, Nr. L 117 vom 5.5.1987, S. 22ff. (Zitierweise: $=$ Rasenmäher-Richtlinie $1987=$ )

Kommission der EG: Vollendung des Binnenmarktes. Annäherung der Sätze und Harmonisierung der Strukturen der indirekten Steuern, DOK KOM (87) 320 endg./2, abgedruckt als Bundestags-Drucksache 11/1321. (Zitierweise: = Harmonisierung indirekter Steuern 1987=)

Kommission der EG: Vorschlag für eine Richtlinie des Rates zur Einführung eines Annäherungsprozesses der Mehrwertsteuer- und Verbrauchsteuersätze, DOK KOM (87) 324 endg./2, abgedruckt als Bundestags-Drucksache 11/1325. (Zitierweise: = Mehrwertsteuer- und Verbrauchsteuersätze $1987=)$ 


\section{Literaturverzeichnis}

Kommission der EG: Beschluß vom 24.6.1988 zur Einsetzung eines Beirats der regionalen und lokalen Gebietskörperschaften, in: Amtsblatt der EG, Nr. L 247 vom 6.9.1988, S. 23ff. (Zitierweise: =Einsetzung eines Beirats $1988=)$

Kommission der EG: Verordnung (EWG) Nr. 3578/88 vom $17.11 .1988 \mathrm{mit}$ Durchführungsbestimmungen zu dem System des automatischen Abbaus der negativen Währungsausgleichsbeträge, in: Amtsblatt der EG, Nr. L 312 vom 18.11.1988, S. P6ff. (Zitierweise: =Negative Währungsausgleichsbeträge $=$ )

Kommission der EG: Bericht zur Wirtschafts- und Währungsunion in der Europäischen Gemeinschaft, Brüssel 1989. (Zitierweise: =Delors-Bericht $1989=)$

Kommission der EG: Die Lage der Landwirtschaft in der Gemeinschaft. Bericht 1988, Brüssel, Luxemburg 1989. (Zitierweise: =Lage der Landwirtschaft $1988=$ )

Kommission der EG: Die öffentlichen Finanzen der Gemeinschaft. Der Gemeinschaftshaushalt nach der Reform von 1988, Brüssel 1989. (Zitierweise: =Offentliche Finanzen=)

Kommission der EG: Entscheidung vom 20.12.1989 über die Festlegung des gemeinschaftlichen Förderkonzepts als Beitrag des Europäischen Sozialfonds zur Erreichung der Ziele Nrn. 3 und 4 in der Bundesrepublik Deutschland, in: Amtsblatt der EG, Nr. L 64 vom 13.3.1990, S. 18f. (Zitierweise: =Gemeinschaftliches Förderkonzept Ziele 3 und 4 1990=)

Kommission der EG: Finanzbericht 1988, Luxemburg 1989. (Zitierweise: $=$ Finanzbericht $1988=$ )

Kommission der EG: Geänderter Vorschlag für eine Richtlinie des Rates über das Wahlrecht der Staatsangehörigen der Mitgliedstaaten bei den Kommunalwahlen im Aufenthaltsstaat, in: Amtsblatt der EG, Nr. C 290 vom 18.11.1989, S. 4ff. (Zitierweise: =Vorschlag Kommunalwahlrecht=)

Kommission der EG: Haushaltsvademekum der Gemeinschaft, Ausgabe 1989, Brüssel, Luxemburg 1989. (Zitierweise: =Haushaltsvademekum 1989=)

Kommission der EG: Jahreswirtschaftsbericht 1989-1990, DOK KOM (89) 497 endg.

Kommission der EG: Leitfaden zur Reform der Strukturfonds der Gemeinschaft, Brüssel, Luxemburg 1989. (Zitierweise: = Reform der Strukturfonds $1989=$ )

Kommission der EG: Mitteilung der Kommission an den Rat. Wirtschaftliche Konvergenz in der EG: Eine größere Anstrengung ist erforderlich, kurzfristige wirtschaftliche Aussichten und Haushaltspolitik 1989/90, DOK KOM (89) 333 endg.

Kommission der EG: Mitteilung der Kommission über ihr Aktionsprogramm zur Anwendung der Gemeinschaftscharta der sozialen Grundrechte, DOK KOM (89) 568 endg. (Zitierweise: =Soziale Grundrechte=) 
Kommission der EG: Abschaffung der Steuergrenzen: Übergangssystem für die Mehrwertsteuer bis zum 31.12.1996, in: EG-Nachrichten, Nr. 5 vom 29.5.1990. (Zitierweise: =Steuergrenzen 1990=)

Kommission der EG: Entscheidung vom 20.12.1989 zur Erstellung des gemeinschaftlichen Förderkonzepts für die Strukturinterventionen der Gemeinschaft für die kreisfreie Stadt Emden, für die Gebiete des Stadtverbandes Saarbrücken und des Landkreises Saarlouis, für Gebiete in der kreisfreien Stadt Salzgitter und im Landkreis Peine, für den Kreis Pirmasens und die kreisfreien Städte Pirmasens und Zweibrücken in RheinlandPfalz, für Berlin, für Gebiete in Nordrhein-Westfalen und für Gebiete des Landes Bremen, in: Amtsblatt der EG, Nr. L 155 vom 21.6.1990, S. $35 \mathrm{ff}$. (Zitierweise: $=$ GFK Ziel 2 1990=)

Kommission der EG: XXIII. Gesamtbericht über die Tätigkeit der Europäischen Gemeinschaften 1989, Brüssel, Luxemburg 1990. (Zitierweise: $=$ XXIII. Gesamtbericht $=$ )

Kommission der EG: Zweiter Bericht über die staatlichen Beihilfen in den Europäischen Gemeinschaften 1989, Brüssel, Luxemburg 1990. (Zitierweise: = Staatliche Beihilfen II=)

Kommission der EG: Änderung des Vorschlags für eine Richtlinie des Rates zur Ergänzung des gemeinsamen Mehrwertsteuersystems und zur Anderung der Richtlinie 77/388/EWG, Steuerliche Ubergangsregelungen im Hinblick auf die Errichtung des Binnenmarktes, DOK KOM (90) 182 endg., abgedruckt in: Bundesrats-Drucksache 510/90. (Zitierweise: =Steuerliche Ubergangsregelung 1990=)

Kommission der EG: Die Europäische Gemeinschaft und die deutsche Vereinigung, in: Bulletin der EG, Beilage 4/90, Luxemburg 1990. (Zitierweise: $=$ Deutsche Vereinigung $1990=$ )

Kommission der EG: Die Gemeinschaft und die deutsche Einigung, DOK KOM (90) 400 endg.

Kommission der EG: Entscheidung vom 21.2.1990 zur Änderung der deutschen Beihilferegelung zugunsten der KfZ-Industrie, in: Amtsblatt der EG, Nr. L 188 vom 20.7.1990, S. 55ff. (Zitierweise: =Beihilferegelung für KfZ-Industrie 1990=)

Kommission der EG: Europäischer Fonds für regionale Entwicklung, Jahresbericht 1988, Luxemburg 1990.

Kommission der EG: Länderstudien, Nummer 1: Bundesrepublik Deutschland, Brüssel 1990. (Zitierweise: =Länderstudien 1=)

Kommission der EG: Agrarpreise 1991/92, Vorschläge der Kommission, in: Grünes Europa, Nr. 1/91, Brüssel 1991. (Zitierweise: = Agrarpreise $1991 / 92=)$

Kommission der EG: Die Lage der Landwirtschaft in der Gemeinschaft, Bericht 1990, Brüssel, Luxemburg 1991. (Zitierweise: = Lage der Landwirtschaft $1990=$ ) 
Kommission der EG: Entscheidung vom 18.7.1991 über Beihilfen der Freien und Hansestadt Hamburg, in: Amtsblatt der EG, Nr. L 215 vom 2.8.1991, S. 1 ff. (Zitierweise: =Beihilfen der Freien und Hansestadt Hamburg=)

Kommission der EG: Jahresbericht zur Durchführung der Strukturfonds 1989, Brüssel 1991. (Zitierweise: = Durchführung der Strukturfonds=)

Kommission der EG: Jahreswirtschaftsbericht 1990/91. Revision, abgedruckt in: EG-Nachrichten, Berichte und Informationen - Dokumentation, Nr. 4 vom 3.6.1991. (Zitierweise: =Jahreswirtschaftsbericht 1990/91 Revision $=$ )

Kommission der EG: Ohne Reform der Agrarpolitik wird der EG-Haushalt außer Kontrolle geraten, in: EG-Informationen, Nr. 3/1991, S. 7ff. (Zitierweise: = Reform der Agrarpolitik=)

Kommission der EG: Vorschläge betreffend die Festsetzung der Preise für landwirtschaftliche Erzeugnisse und bestimmte flankierende Maßnahmen (1991/92), in: Amtsblatt der EG, Nr. C 104 vom 19.4.1991. (Zitierweise: $=$ Preisvorschläge 1991/92)

Krieger, Christiane; Thoroe, Carstens; Weskamp, Wolfgang: Regionales Wirtschaftswachstum und sektoraler Strukturwandel in der Europäischen Gemeinschaft, Tübingen 1985. (Zitierweise: = Regionales Wirtschaftswachstum =)

Krieger-Boden, Christiane: Zur Regionalpolitik der Europäischen Gemeinschaft, in: Weltwirtschaft, (1987), S. 82ff. (Zitierweise: = Regionalpolitik $\operatorname{der} E G=)$

Krueger, Anne O.: Eisernes Dreieck. Staatsversagen statt Marktversagen, in: Wirtschaftswoche, Nr. 50 vom 7.12. 1990, S. 88ff.

Kuschel, Hans-Dieter: Die Einbeziehung der ehemaligen DDR in die Europäische Gemeinschaft, in: Wirtschaftsdienst, 71. Jg. (1991), S. 80ff. (Zitierweise: =Einbeziehung $=$ )

Lammers, Konrad: Mehrfachsubventionen (EG, Bund, Länder, Gemeinden, Sondervermögen, KW, LAB). Generelle Problematik und Vorschläge zum Abbau, Kiel 1984.

Langes, Horst: Die EG ist kein Privatunternehmen der Außenminister, in: Frankfurter Allgemeine Zeitung vom 1.3.1991, S. 16. (Zitierweise: =EG kein Privatunternehmen=)

Lemper, Alfons: Europäische Integration als gruppendynamischer Prozeß, in: List-Forum, Band 16 (1990), S. $21 \mathrm{ff}$.

Lenz, Carl O.: Die Verkehrspolitik der Europäischen Gemeinschaften im Lichte der Rechtsprechung des Gerichtshofes, in: Europarecht, 23. Jg. (1988), S. 158ff. (Zitierweise: =Verkehrspolitik der EG $=$ )

Liedtke, Cornelius: Theorie der öffentlichen Güter und optimale Struktur einer Föderation, Berlin 1972. (Zitierweise: =Optimale Stuktur einer Föderation =) 
Matthies, Heinrich: Grundlagen des Subventionsrechtes. Kompetenzen aus EG-rechtlicher Sicht, in: Zeitschrift für das gesamte Handels- und Wirtschaftsrecht, Band 152 (1988), S. 442ff. (Zitierweise: =Subventionsrecht =)

May, Bernhard: Kosten und Nutzen der deutschen EG-Mitgliedschaft, Bonn 1982.

Messal, Rüdiger: Das neue Finanzierungssystem der Europäischen Gemeinschaft, in: Wirtschaftsdienst, 68. Jg. (1988), S. 210ff. (Zitierweise: =Das neue Finanzierungssystem $=$ )

Messal, Rüdiger: EG-Finanzierung und Lastenverteilung. Die Reform des EGFinanzierungssystems 1988, in: Schriftenreihe des Bundesministeriums der Finanzen, Heft 42, Bonn 1989. (Zitierweise: =EG-Finanzierung=)

Mohr, Elske: Die Finanzprobleme der Gemeinschaft, in: IFO-Schnelldienst, 33. Jg. (1980), S. 5ff. (Zitierweise: =Finanzprobleme=)

Münch, Rainer: Die Kumulation offener und versteckter Verbindlichkeiten im EG-Haushalt, in: Wirtschaftsdienst, 16. Jg. (1987), S. 97ff. (Zitierweise: $=$ Verbindlichkeiten im EG-Haushalt $=$ )

Münster, Siegfried: Keine Chance gegen die Mark. Ein schwacher Entwurf der europäischen Währungsunion, in: Süddeutsche Zeitung vom 25.8.1990, abgedruckt in: Auszüge aus Presseartikeln, hrsg. von der Deutschen Bundesbank, Nr. 66 vom 29.8.1990, S. 1f. (Zitierweise: = Schwacher Entwurf=)

Musgrave, Richard A.; Musgrave, Peggy B.; Kullmer, Lore: Die öffentlichen Finanzen in Theorie und Praxis, Band 1, 5., überarbeitete Auflage, Tübingen 1990. (Zitierweise: =Offentliche Finanzen 1=)

Musgrave, Richard A.: The Theory of Public Finance, A Study in Public Economy, New York u. a. 1959. (Zitierweise: =Theory of Public Finance=)

Nam, Ch. W.; Nerb, G.; Renter, J.; Russ, H.: Wettbewerbsfähigkeit ausgewählter EG-Regionen, in: IFO-Schnelldienst Nr. 9/90, S. 10ff. (Zitierweise: $=$ Wettbewerbsfähigkeit $=$ )

von Nell-Breuning, Oswald: Subsidiaritätsprinzip, in: Staatslexikon, Bd. 7, 6 , völlig neu bearbeitete und erweiterte Auflage, Freiburg 1962, S. $826 \mathrm{ff}$. (Zitierweise: =Subsidiaritätsprinzip=)

Neumann, Manfred: Zur ökonomischen Theorie des Föderalismus, in: Kyklos, Vol. 24 (1971), S. 493ff. (Zitierweise: = Föderalismus=)

Neumann, Manfred J. M.: Eine zweite Stufe der europäischen Wirtschaftsund Währungsunion, in: Wirtschaftsdienst, 70. Jg. (1990), S. 335ff. (Zitierweise: $=$ Zweite Stufe =)

Neumann, Manfred J. M.: Gemeinsame Aufgaben. Die EG-Regierungskonferenz sollte Leitlinien für ordnungspolitische Reformen in den Mitgliedsländern verabschieden, in: Wirtschaftswoche, Nr. 46 vom 9.11.1990, S. 85ff. (Zitierweise: =Gemeinsame Aufgaben=) 
Neumark, Fritz: Grundsätze gerechter und ökonomisch rationaler Steuerpolitik, Tübingen 1970. (Zitierweise: = Steuerpolitik=)

Niskanen, William A.: Bureaucracy and Representive Government, Chicago 1971.'(Zitierweise: =Bureaucracy $=$ )

Nittka, Ulrich: Das Finanzierungssystemn der Europäischen Gemeinschaften, Bochum 1979. (Zitierweise: =Finanzierungssystem =)

Noé, Claus: Wo sind 19,5 Milliarden DM geblieben? Bemerkungen zu Zielen, Mitteln und Wirkungsweise regionalpolitischer Versuche der Europäischen Gemeinschaft, in: Raumforschung und Raumordnung, 41. Jg. (1983), S. 15ff. (Zitierweise: = Regionalpolitische Versuche=)

Nottelmann, Angela: Der Haushalt der Europäischen Gemeinschaften. Probleme und Reformvorstellungen, Hamburg 1982. (Zitierweise: = Haushalt der $\mathrm{EG}=)$

Oates, Wallace E.: Ein ökonomischer Ansatz zum Föderalismusproblem, in: Föderalismus, hrsg. von Guy Kirsch, Stuttgart, New York 1977, S. 15ff. (Zitierweise: $=$ Föderalismusproblem $=$ )

Oates, Wallace E.: Finanzföderalismus (Fiscal Federalism) in Theorie und Praxis: Lehren für die Europäische Gemeinschaft, in: Bericht der Sachverständigengruppe zur Untersuchung der Rolle der öffentlichen Finanzen bei der Europäischen Integration, Band II, hrsg. von der Kommission der EG, Brüssel 1977, S. 291ff. (Zitierweise: =Finanzföderalismus=)

Oates, Wallace E.: Public Finance in a Federal System, in: Canadian Journal of Economics, Vol. 1 (1968), S. 37ff. (Zitierweise: =Federal System=)

Olson, Mancur: Strategic Theory and its Applications. The Principle of "Fiscal Equivalence": The Division of Responsibilities Among Different Levels of Government, in: American Economic Review, Vol. 59 (1969), Papers and Proceedings, S. 479ff. (Zitierweise: =Fiscal Equivalence=)

Olson, Mancur; Zeckhauser, Richard: An Economic Theory of Alliances, in: The Review of Economics and Statistics, Vol. 48 (1966), S. $266 \mathrm{ff}$. (Zitierweise: $=$ Theory of Alliances $=$ )

Oschatz, Georg-Berndt; Risse, Horst: Europäische Integration und deutscher Föderalismus, in: Zeitschrift für internationale Politik, Jg. 43 (1988), S. 9ff. (Zitierweise: =Deutscher Föderalismus=)

Ott, Günter: Internationale Verteilungswirkungen im Finanzausgleich der Europäischen Gemeinschaft, Frankfurt a. M. u. a. 1987. (Zitierweise: =Verteilungswirkungen $=$ )

O. V.: Mit einer EG-Kammer will der Bundesrat auf die Europäische Integration einwirken, in: Frankfurter Allgemeine Zeitung vom 27.5.1988, S. 5. (Zitierweise: =EG-Kammer $=$ )

O. V.: EG schafft Voraussetzungen für liberalisierten Verkehrsmarkt, in: Frankfurter Allgemeine Zeitung vom 16.3.1989, S. 17. (Zitierweise: $=\mathrm{Li}$ beralisierter Verkehrsmarkt $=$ ) 
O. V.: Hessen eröffnet Europa-Büro in Brüssel, in: Frankfurter Allgemeine Zeitung vom 20.4.1989, S. 6. (Zitierweise: =Europa-Büro=)

O. V.: Die Pfadfinder im EG-Labyrinth. Staatliche und private Lobbyisten rings um die Kommission, in: Frankfurter Allgemeine Zeitung vom 3.10.1989, S. 6. (Zitierweise: = Pfadfinder im EG-Labyrinth=)

O. V.: Wachsender Unmut im EG-Verkehrsministerrat, in: Frankfurter Allgemeine Zeitung vom 17.10.1989, S. 17. (Zitierweise: =EG-Verkehrsministerrat $=$ )

O. V.: Die Bundesregierung plant abermals Novellierung der Trinkwasserverordnung, in: Frankfurter Allgemeine Zeitung vom 10.1.1990, S. 4. (Zitierweise: = Trinkwasserverordnung $=$ )

O. V.: EG liberalisiert die Vergabe öffentlicher Aufträge ab 1993. Keine Bevorzugung inländischer Anbieter bei Ausschreibungen, in: Deutsche Sparkassenzeitung vom 27.2.1990, S. 2. (Zitierweise: = Offentliche Aufträ$\mathrm{ge}=$ )

O. V.: Die Bundesländer wollen mitreden, in: Frankfurter Allgemeine Zeitung vom 17.12.1990, S. 2. (Zitierweise: =Bundesländer $=$ )

O. V.: Mehrheit in der EG gegen grundlegende Änderungen der Zuständigkeiten, in: Frankfurter Allgemeine Zeitung vom 5.3.1991, S. 2. (Zitierweise: $=$ Zuständigkeiten $=$ )

O. V.: Betrügereien mit EG-Geldern, in: Frankfurter Allgemeine Zeitung vom 26.3.1991, S. 18. (Zitierweise: =Betrügereien=)

O. V.: Brüssel fordert eigene "Europa-Steuer", in: Frankfurter Allgemeine Zeitung vom 23.5.1991, S. 15. (Zitierweise: '=Europa-Steuer $=$ )

O. V.: Europäische Steuerangleichung mit Ausnahmen, in: Frankfurter Allgemeine Zeitung vom 26.6.1991, S. 15 und 17. (Zitierweise: =Europäische Steuerangleichung $=$ )

Pag, Sabine: Alltagsgeschäft EG. Die Umsetzung von EG-Vorschriften in der Bundesrepublik Deutschland, in: Integration, 11. Jg. (1988), S. 127ff. (Zitierweise: =Umsetzung von EG-Vorschriften=)

Parsche, Rüdiger; Seidel, Bernhard; Teichmann, Dieter: Die Beseitigung von Steuergrenzen in der Europäischen Gemeinschaft. Vorteile und Probleme einer Harmonisierung von Mehrwertsteuer und Verbrauchsteuern im europäischen Binnenmarkt, Berlin 1988. (Zitierweise: = Beseitigung von Steuergrenzen $=$ )

Peffekoven, Rolf: Finanzausgleich I. Wirtschaftstheoretische Grundlagen, in: Handwörterbuch der Wirtschaftswissenschaften, Band 2, hrsg. von Willi Albers et al., Tübingen 1980, S. 608ff. (Zitierweise: = Finanzausgleich $\mathrm{I}=$ ) 
Peffekoven, Rolf: Probleme der internationalen Finanzordnung, in: Handbuch der Finanzwissenschaft, hrsg. von Fritz Neumark unter Mitwirkung von Norbert Andel und Heinz Haller, Band 4, 3., gänzlich neubearbeitete Auflage, Tübingen 1983, S. 219ff. (Zitierweise: =Internationale Finanzordnung =)

Peffekoven, Rolf: Eigene Einnahmen internationaler Organisationen, in: Staat, Steuer und Finanzausgleich, Festschrift für Heinz Kolms zum 70. Geburtstag, hrsg. von Walter A. S. Koch und Hans-Georg Petersen, Berlin 1984, S. 315ff. (Zitierweise: =Eigene Einnahmen=)

Pennock, R. J.: Federal and Unitary Government-Disharmony and Frustration, in: Behavioural Science, Vol. IV (1959), S. 147ff. (Zitierweise: $=$ Government-Disharmony and Frustration $=$ )

Petersen, Volker: Agrarpolitik und EG-Haushalt. Gemeinsame Agrarpolitik und der Haushalt der Europäischen Gemeinschaft, München-Hiltrup 1983. (Zitierweise: $=$ Agrarpolitik=)

Pfennig, Gero: Eine neue Finanzverfassung für die EG. Eine Basis für eine föderative Europäische Union, in: Integration, Jg. 9 (1986), S. $143 \mathrm{ff}$. ( $\mathrm{Zi}$ tierweise: =Finanzverfassung=)

Pflugmann, Barbara: Zum Abbau der Steuergrenzen in den Europäischen Gemeinschaften, in: Finanzarchiv, N. F., Band 47 (1989), S. $324 \mathrm{ff}$. (Zitierweise: =Steuergrenzen $=$ )

Philipp, Otmar: Probleme bei der Umsetzung der EG-Richtlinien in nationales Recht, in: EG-Magazin, Nr. 11/88, S. 12. (Zitierweise: =Umsetzung von EG-Richtlinien=)

Planungsausschuß für regionale Wirtschaftsstruktur: Sechzehnter Rahmenplan der Gemeinschaftsaufgabe "Verbesserung der regionalen Wirtschaftsstruktur" für den Zeitraum 1987 bis 1990 (1991), in: Bundestagsdrucksache 11/583. (Zitierweise: $=16$. Rahmenplan=)

Planungsausschuß für regionale Wirtschaftsstruktur: Achtzehnter Rahmenplan der Gemeinschaftsaufgabe "Verbesserung der regionalen Wirtschaftsstruktur" für den Zeitraum 1989 bis 1992 (1993), in: Bundestagsdrucksache 11/5099. (Zitierweise: $=18$. Rahmenplan=)

Pohmer, Dieter: Leistungsfähigkeitsprinzip und Einkommensverteilung, in: Theorie und Praxis des finanzpolitischen Interventionismus, Fritz Neumark zum 70. Geburtstag, hrsg. von Heinz Haller et al., Tübingen 1970, S. 135ff. (Zitierweise: =Leistungsfähigkeitsprinzip=)

Pohmer, Dieter: Zum Grenzausgleich bei der Umsatzsteuer, in: Wirtschaftswissenschaft als Grundlage staatlichen Handelns, Heinz Haller zum 65. Geburtstag hrsg. von Peter Bohley und Georg Tolkemitt, Tübingen 1979, S. 249ff. (Zitierweise: = Grenzausgleich $=$ )

Pohmer, Dieter: Allgemeine Umsatzsteuern, in: Handbuch der Finanzwissenschaft, hrsg. von Fritz Neumark unter Mitwirkung von Norbert Andel und Heinz Haller, Band II, 3. Auflage, Tübingen 1980, S. 647ff. (Zitierweise: $=$ Allgemeine Umsatzsteuern $=$ ) 
Pohmer, Dieter: Zur Systematik und Praxis der Mehrwertsteuern unter besonderer Berücksichtigung der Entwicklung in Europa, in: Unternehmung und Steuern, Festschrift zur Vollendung des 80. Lebensjahres von Peter Scherpf, hrsg. von Lutz Fischer, Wiesbaden 1983, S. 375ff.

Popitz, Johannes: Der Finanzausgleich, in: Handbuch der Finanzwissenschaft, hrsg. von Wilhelm Gerloff und Franz Meisel, Band II, 1. Auflage, Tübingen 1927, S. 338ff. (Zitierweise: =Finanzausgleich =)

Presse- und Informationsamt der Bundesregierung: Einbeziehung der ehemaligen DDR in die Europäische Gemeinschaft, in: Aktuelle Beiträge zur Wirtschafts- und Finanzpolitik, Nr. 8/1991 vom 4.3.1991. (Zitierweise: $=$ Einbeziehung $=$ )

Presse- und Informationsamt der Bundesregierung: Noch knapp zwei Jahre bis zur Vollendung des Binnenmarktes 1992, Stand und weitere Aussichten, in: Aktuelle Beiträge zur Wirtschafts- und Finanzpolitik, Nr. 9/1991 vom 4.3.1991. (Zitierweise: =Stand und Aussichten=)

Priebe, Hermann: Die subventionierte Unvernunft. Landwirtschaft und Naturhaushalt, Berlin 1985. (Zitierweise: =Subventionierte Unvernunft $=$ )

Püttner, Günter; Spannowsky, Willy: Das Verhältnis der europäischen Regionalpolitik zur deutschen Regionalpolitik, Bonn 1986. (Zitierweise: $=$ Verhältnis europäischer zu deutscher Regionalpolitik =)

Rat der EG: Verordnung (EWG) Nr. 1612/68 vom 15.10.1968 über die Freizügigkeit der Arbeitnehmer innerhalb der Gemeinschaft, in: Amtsblatt der EG, Nr. L 257 vom 19.10.1968, S. 2ff. (Zitierweise: =Freizügigkeit $1968=$ )

Rat der EG: Beschluß vom 21.4.1970 über die Ersetzung der Finanzbeiträge der Mitgliedstaaten durch eigene Mittel der Gemeinschaft, in: Amtsblatt der EG, Nr. L 94 vom 28.4.1970, S. 19ff. (Zitierweise: =Eigenmittelbeschluß $1970=$ )

Rat der EG: Entscheidung vom 18.2.1974 zur Erreichung eines hohen Grades an Konvergenz der Wirtschaftspolitik der Mitgliedstaaten der Europäischen Wirtschaftsgemeinschaft, in: Amtsblatt der EG, Nr. L 63 vom 5.3.1974, S. 16ff. (Zitierweise: =Konvergenz 1974=)

Rat der EG: Richtlinie vom 16.6.1975 über die Qualitätsanforderungen an Oberflächenwasser für die Trinkwassergewinnung in den Mitgliedstaaten, in: Amtsblatt der EG, Nr. L 194 vom 25.7.1975, S. 34ff. (Zitierweise: $=$ Qualitätsanforderungen 1975=)

Rat der EG: Richtlinie vom 4.5.1976 betreffend die Verschmutzung infolge der Ableitung bestimmter gefährlicher Stoffe in die Gewässer der Gemeinschaft, in: Amtsblatt der EG, Nr. L 129 vom 4.5.1976, S. 23ff. (Zitierweise: =Ableitung gefährlicher Stoffe 1976=)

Rat der EG: Sechste Richtlinie vom 17.5.1977 zur Harmonisierung der Rechtsvorschriften der Mitgliedstaaten über die Umsatzsteuern - Gemeinsames Mehrwertsteuersystem: einheitliche steuerpflichtige Bemessungsgrundlage, in: Amtsblatt der EG, Nr. L 145 vom 13.6.1977, S. 1ff. (Zitierweise: =Sechste Richtlinie zur Umsatzsteuerharmonisierung=) 
Rat der EG: Richtlinie vom 25.7.1977 über die schulische Betreuung der Kinder von Wanderarbeitnehmern, in Amtsblatt der EG, Nr. L 199 vom 16.8.1977, S. 32ff. (Zitierweise: =Kinder von Wanderarbeitnehmern $1976=$ )

Rat der EG: Verordnung (EWG) Nr. 1079/77 vom 17.5.1977 über eine Mitverantwortungsabgabe und Maßnahmen zur Erweiterung der Märkte für Milch und Milcherzeugnisse, in: Amtsblatt der EG, Nr. L 131 vom 26.5.1977, S. 6f. (Zitierweise: = Mitverantwortungsabgabe für Milch $1977=)$

Rat der EG: Richtlinie vom 17.12.1979 über den Schutz des Grundwassers gegen Verschmutzung durch bestimmte gefährliche Stoffe, in: Amtsblatt der EG, Nr. L 20 vom 26.1.1980, S. 43ff. (Zitierweise: =Schutz des Grundwassers $1979=$ )

Rat der EG: Richtlinie vom 15.7.1980 über Grenzwerte und Leitwerte der Luftqualität für Schwefeldioxid und Schwebstaub, in: Amtsblatt der EG, Nr. $L 229$ vom 30.8.1980, S. 30f. (Zitierweise: = Grenzwerte der Luftqualität $1980=$ )

Rat der EG: Richtlinie vom 15.7.1980 über die Qualität von Wasser für den menschlichen Gebrauch in: Amtsblatt der EG, Nr. L 229 vom 30.8.1980, S. 11ff. (Zitierweise: = Wasserqualität 1980=)

Rat der EG: Richtlinie vom 15.7.1980 zur Änderung der Richtlinien, mit denen die Grundnormen für den Gesundheitsschutz der Bevölkerung und der Arbeitskräfte gegen die Gefahren ionisierender Strahlungen festgelegt wurden, in: Amtsblatt der EG, Nr. L 246 vom 17.9.1980, S. 1ff. (Zitierweise: = Richtlinie zum Strahlenschutz 1980=)

Rat der EG: Verordnung (EWG) Nr. 1785/81 vom 30.6.1981 über die gemeinsame Marktorganisation für Zucker, in: Amtsblatt der EG, Nr. L 777 vom 1.7.1981, S. 4ff. (Zitierweise: = Marktorganisation für Zucker $1981=)$

Rat der EG: Verordnung (EWG) Nr. 856/84 vom 31.3.1984 zur Änderung der Verordnung (EWG) Nr. 804/68 über die gemeinsame Marktorganisation für Milch und Milcherzeugnisse, in: Amtsblatt der EG, Nr. L 90 vom 1.4.1984, S. 10ff. (Zitierweise: = Marktorganisation für Milch $1984=$ )

Rat der EG: Verordnung (EWG) Nr. 1787/84 vom 19.6.1984 betreffend den Europäischen Fonds für regionale Entwicklung, in: Amtsblatt der EG, Nr. L 169 vom 28.6.1984, S. 1ff. (Zitierweise: =EFRE-Verordnung 1984=)

Rat der EG: Beschluß vom 7.5.1985 über das System der eigenen Mittel der Gemeinschaften, in: Amtsblatt der EG, Nr. L 128 vom 14.5.1985, S. $15 \mathrm{ff}$. (Zitierweise: =Eigenmittelbeschluß 1985=)

Rat der EG: Entscheidung yom 7.5.1985 über eine neue Konzeption auf dem Gebiet der technischen Harmonisierung und Normung, in: Amtsblatt der EG, Nr. C 136 vom 4.6.1985, S. 1ff. (Zitierweise: =Technische Harmonisierung und Normung $1985=$ ) 
Rat der EG: Verordnung (EWG) Nr. 2088/85 vom 23.7.1985 über die integrierten Mittelmeerprogramme, in: Amtsblatt der EG, Nr. L 197 vom 27.7.1985, S. 1ff. (Zitierweise: =Integrierte Mittelmeerprogramme $1985=$ )

Rat der EG: Verordnung (EWG) Nr. 3820/85 vom 20.12.1985 über die Harmonisierung bestimmter Sozialvorschriften im Straßenverkehr, in: Amtsblatt der EG, Nr. L 370 vom 31.12.1985, S. 1ff. (Zitierweise: =Sozialvorschriften im Straßenverkehr 1985=)

Rat der EG: Verordnung (EWG) Nr. 1579/86 vom 23.5.1986 zur Änderung der Verordnung (EWG) Nr. $2727 / 75$ über die gemeinsame Marktorganisation für Getreide, in: Amtsblatt der EG, Nr. L 139 vom 24.5.1986, S. 29ff. (Zitierweise: = Marktorganisation für Getreide 1986=)

Rat der EG: Verordnung (EWG) Nr. 1983/86 vom 24.6.1986 zur Festlegung von Grundregeln für die direkte Beihilfenregelung zugunsten der Kleinerzeuger im Getreidesektor, in: Amtsblatt der EG, Nr. L 171 vom 28.6.1986, S. 1ff. (Zitierweise: =Kleinerzeuger im Getreidesektor $1986=)$

Rat der EG: Beschluß vom 9.3.1987 zur Ermächtigung der Kommission im Rahmen des Neuen Gemeinschaftsinstruments Anleihen zur Investitionsförderung in der Gemeinschaft aufzunehmen, in: Amtsblatt der EG, Nr. L 71 vom 14.3.1987, S. 34ff. (Zitierweise: = Neues Gemeinschaftsinstrument =)

Rat der EG: Beschluß vom 28.9.1987 über das gemeinschaftliche Rahmenprogramm im Bereich der Forschung und technologischen Entwicklung (19871991), in: Amtsblatt der EG, Nr. L 302 vom 24.10.1987, S. 1ff. (Zitierweise: = Rahmenprogramm 1987-1991=)

Rat der EG: Entschließung des Rates vom 19.10.1987 zur Fortschreibung und Durchführung einer Umweltpolitik und eines Umweltaktionsprogramms der Europäischen Gemeinschaften für den Umweltschutz (1987-1992), in: Amtsblatt der EG, Nr. L 328 vom 7.12.1987, S. 1ff. (Zitierweise: =Aktionsprogramm Umweltschutz 1987-1992=)

Rat der EG: Beschluß vom 24.6.1988 über das System der Eigenmittel der Gemeinschaften in: Amtsblatt der EG, Nr. L 185 vom 15.7.1988, S. $19 \mathrm{ff}$. (Zitierweise: =System der Eigenmittel 1988=)

Rat der EG: Entscheidung vom 24.5.1988 betreffend die Haushaltsdisziplin, in: Amtsblatt der EG, Nr. L 185 vom 15.7.1988, S. 29ff. (Zitierweise: $=$ Haushaltsdisziplin $1988=$ )

Rat der EG: Entscheidung vom 25.7.1988 zur Annahme eines MehrjahresForschungs- und Ausbildungsprogramms auf dem Gebiet der kontrollierten Kernfusion, in: Amtsblatt der EG, Nr. L 222 vom 12.8.1988, S.5ff. (Zitierweise: =Kontrollierte Kernfusion 1988=)

Rat der EG: Interinstitutionelle Vereinbarung über die Haushaltsdisziplin und die Verbesserung des Haushaltsverfahrens, in: Amtsblatt der EG, $\mathrm{Nr}$. L 185 vom 15.7.1988, S. 33ff. (Zitierweise: =Interinstitutionelle Vereinbarung $1988=$ ) 


\section{Literaturverzeichnis}

Rat der EG: Richtlinie zur Harmonisierung der Anforderungen zum Schutz von Sicherheit und Gesundheit für den Verkehr mit Spielzeug, in: Amtsblatt der EG, Nr. L 187 vom 16.7.1988, S. 1ff. (Zitierweise: = SpielzeugRichtlinie $1988=$ )

Rat der EG: Richtlinie vom 21.12.1988 über eine allgemeine Regelung zur Anerkennung der Hochschuldiplome, die eine mindestens dreijăhrige $\mathrm{Be}-$ rufsausbildung abschließen, in: Amtsblatt der EG, Nr. L 19 vom 24.1.1989, S. 16ff. (Zitierweise: = Anerkennung von Hochschuldiplomen $1988=$ )

Rat der EG: Verordnung (EWG) Nr. 328/88 vom 2.2.1988 zur Einführung eines Gemeinschaftsprogramms zugunsten der Umstellung von Eisen- und Stahlrevieren (Programm RESIDER), in: Amtsblatt der EG, Nr. L 33 vom 5.2.1988, S. 1ff. (Zitierweise: = RESIDER-Programm 1988=)

Rat der EG: Verordnung (EWG) Nr. 1094/88 vom 25.4.1988 zur Änderung der Verordnung (EWG) Nr. 795/85 und Nr. 1760/87 hinsichtlich der Stilllegung von Ackerflächen und der Extensivierung und Umstellung der Erzeugung, in: Amtsblatt der EG, Nr. L 106 vom 27.4 .1988 , S. 28ff. (Zitierweise: =Stillegung von Ackerflächen $1988=$ )

Rat der EG: Verordnung (EWG) Nr. 1097/88 vom 25.4.1988 zur Änderung der Verordnung (EWG) Nr. $2727 / 75$ über die gemeinsame Marktorganisation für Getreide, in: Amtsblatt der EG, Nr. L 110 vom 29.4.1988, S. 7ff. (Zitierweise: = Marktorganisation für Getreide 1988=)

Rat der EG: Verordnung (EWG) Nr. 2052/88 vom 24.6.1988 über Aufgaben und Effizienz der Strukturfonds und über die Koordinierung ihrer Interventionen untereinander sowie mit denen der Europäischen Entwicklungsbank und der anderen vorhandenen Finanzinstrumente, in: Amtsblatt der EG, Nr. L 185 vom 15. Juli 1988, S. 9ff. (Zitierweise: = Aufgaben und Effizienz der Strukturfonds 1988=)

Rat der EG: Verordnung (EWG) Nr. 2053/88 vom 24.6.1988 über einen finanziellen Beitrag zugunsten Portugals für ein Sonderprogramm zur industriellen Entwicklung (PEDIP), in: Amtsblatt der EG, Nr. $\mathrm{L} 185 \mathrm{vom}$ 15.7.1988, S. 21ff. (Zitierweise: =Verordnung über PEDIP 1988=)

Rat der EG: Richtlinie vom 24.11.1988 zur Begrenzung von Schadstoffemissionen von Großfeuerungsanlagen in die Luft, in: Amtsblatt der EG, Nr. L 336 vom 7.12.1988, S. $1 \mathrm{ff}$. (Zitierweise: =Schadstoffemissionen von Großfeuerungsanlagen $1988=$ )

Rat der EG: Verordnung (EWG) Nr. 4253/88 vom 19.12.1988 zur Durchführung der Verordnung (EWG) Nr. 2052/88 hinsichtlich der Koordinierung der Interventionen der verschiedenen Strukturfonds einerseits und zwischen diesen und den Interventionen der Europäischen Investitionsbank und der sonstigen vorhandenen Finanzinstrumente andererseits, in: Amtsblatt der EG, Nr. L 374 vom 31.12.1988, S. 1f. (Zitierweise: = Koordinierung der Strukturfonds $1988=$ ) 
Rat der EG: Entscheidung vom 20.6.1989 zur Annahme eines Forschungsund Ausbildungsprogramms der Europäischen Atomgemeinschaft auf dem Gebiet des Strahlenschutzes (1990-1991), in: Amtsblatt der EG, Nr. L 200 vom 13.7.1989, S. 50ff. (Zitierweise: =Strahlenschutzprogramm 1990$1991=$ )

Rat der EG: Entscheidung vom 20.11.1989 über zwei spezifische Programme für Forschung und technologische Entwicklung im Bereich Umwelt: STEP und EPOCH (1989-1992), in: Amtsblatt der EG, Nr. L 359 vom 8.12.1989, S. 9ff. (Zitierweise: = STEP und EPOCH 1989=)

Rat der EG: Richtlinie vom 13.2.1989 zur Harmonisierung der Erfassung des Bruttosozialprodukts zu Marktpreisen, in: Amtsblatt der EG, $\mathrm{Nr}$. L 49 vom 21.2.1989, S. 26ff. (Zitierweise: =Erfassung des BSP MP $1989=)$

Rat der EG: Achtzehnte Richtlinie des Rates zur Harmonisierung der Rechtsvorschriften über die Umsatzsteuern, in: Amtsblatt der EG, Nr. L 226 vom 18.7.1989, S. 21ff. (Zitierweise: = Achtzehnte Richtlinie zur Umsatzsteuerharmonisierung $=$ )

Rat der EG: Richtlinie zur Änderung der Richtlinie 71/305/EWG über die Koordination der Verfahren zur Vergabe öffentlicher Bauaufträge, in: Amtsblatt der EG, Nr. L 210 vom 21.7.1989, S. 1ff. (Zitierweise: =Vergabe öffentlicher Bauaufträge 1989=)

Rat der EG: Richtlinie vom 3.10.1989 zur Koordinierung bestimmter Rechtsund Verwaltungsvorschriften der Mitgliedstaaten über die Ausübung der Fernsehtätigkeit, in: Amtsblatt der EG, Nr. L 298 vom 17.10.1989, S. 23ff. (Zitierweise: =Fernseh-Richtlinie 1989=)

Rat der EG: Verordnung (EWG) Nr. 768/89 vom 21.3.1989 zur Einführung vorübergehender landwirtschaftlicher Einkommensbeihilfen, in: Amtsblatt der EG, Nr. L 84 vom 29.3.1989, S. 8ff. (Zitierweise: = Einkommensbeihilfen $1989=$ )

Rat der EG: Verordnung (Euratom) Nr. 2218/89 vom 18.7.1989 zur Änderung der Verordnung (Euratom) Nr. 3954/87 zur Festlegung von Höchstwerten an Radioaktivität in Nahrungsmitteln und Futtermitteln im Falle eines nuklearen Unfalls oder einer anderen radiologischen Notstandssituation, in: Amtsblatt der EG, Nr. L 211 vom 22.7.1989, S. 1ff. (Zitierweise: $=$ Höchstwerte an Radioaktivität 1989=)

Rat der EG: Verordnung (EWG) Nr. 3906/89 über Wirtschaftshilfe für die Republik Ungarn und die Volksrepublik Polen, in: Amtsblatt der EG, Nr. L 375 vom 23.12.1989, S. 11ff. (Zitierweise =Wirtschaftshilfe für Ungarn und Polen 1989=)

Rat der EG: Beschluß vom 12.3.1990 zur Änderung des Beschlusses 64/300/EWG über die Zusammenarbeit zwischen den Zentralbanken der Mitgliedstaaten der Europäischen Wirtschaftsgemeinschaft, in: Amtsblatt der EG, Nr. L 78 vom 24.3.1990, S. 25f. (Zitierweise: =Zusammenarbeit zwischen den Zentralbanken 1990=) 
Rat der EG: Entscheidung vom 12.3.1990 zur Erreichung einer schrittweisen Konvergenz der Politiken und der wirtschaftlichen Ergebnisse während der ersten Stufe der Wirtschafts- und Währungsunion, in: Amtsblatt der EG Nr. L 78 vom 24.3.1990, S. 23ff. (Zitierweise: =Schrittweise Konvergenz $1990=$ )

Rat der EG: Richtlinie vom 28.6.1990 über das Aufenthaltsrecht, in: Amtsblatt der EG, Nr. L 180 vom 13.7.1990, S. 26f. (Zitierweise: =Aufenthaltsrecht $1990=$ )

Rat der EG: Richtlinie vom 23.7.1990 über das gemeinsame Steuersystem für Fusionen, Spaltungen, die Einbringung von Unternehmensteilen und den Austausch von Anteilen, die Gesellschaften verschiedener Mitgliedstaaten betreffen, in: Amtsblatt der EG, Nr. L 225 vom 20.8.1990, S. Iff. (Zitierweise: =Fusionsrichtlinie $1990=$ )

Rat der EG: Richtline vom 23.7.1990 über das gemeinsame Steuersystem der Mutter- und Tochtergesellschaft verschiedener Mitgliedstaaten, in: Amtsblatt der EG, Nr. L 225 vom 20.8.1990, S. 6ff. (Zitierweise: =Konzernrichtlinie $1990=$ )

Rat der EG: Schlußfolgerungen vom 3.12.1990 zur Mehrwertsteuer-Übergangsregelung, Brüssel 1990. (Zitierweise: = Mehrwertsteuer-Übergangsregelung $1990=$ )

Rat der EG; Kommission der EG: Beschluß des Rates und der Kommission vom 25.2.1991 über den Abschluß des Vierten AKP-EWG-Abkommens, in: Amtsblatt der EG, Nr. L 229 vom 17.8.1991, S. 1ff. (Zitierweise: $=$ Viertes AKP-EWG-Abkommen=)

Rauscher, Anton; Hollerbach, Alexander: Subsidiarität, in: Staatslexikon, Bd. 5, 7., völlig neu bearbeitete Auflage, Freiburg, Basel, Wien 1989, S. $386 \mathrm{ff}$.

Reding, Kurt: Zur Problematik eines Finanzausgleichs in der Europäischen Gemeinschaft, in: Möglichkeiten und Grenzen einer Europäischen Union, Band 2, hrsg. von Hans von der Groeben und Hans Möller, Baden-Baden 1976, S. 199ff. (Zitierweise: = Problematik eines Finanzausgleichs $=$ )

Reister, Erwin: Haushalt und Finanzen der Europäischen Gemeinschaft, Baden-Baden 1975. (Zitierweise: = Haushalt der EG=)

Rengeling, Hans-Werner: Grundlagen des Subventionsrechts und Kompetenzen aus der Sicht von Bund und Ländern, in: Zeitschrift für das gesamte Handels- und Wirtschaftsrecht, Band 152 (1988), S. 455ff. (Zitierweise: $=$ Subventionsrecht $=$ )

Renzsch, Wolfgang: Deutsche Länder und Europäische Integration. Kompetenzverluste und neue Handlungschancen in einem "Europa der Regionen", in: Aus Politik und Zeitgeschichte, Beilage zur Wochenzeitschrift "Das Parlament", B 28/90 vom 6.7.1990,'S. 28ff. (Zitierweise: = Deutsche Länder und europäische Integration $=$ ) 
Rösslein, Thomas: Die Auswirkungen der europäischen Integration auf die Landesparlamente in der Bundesrepublik Deutschland, in: Auswirkungen eines EG-Beitritts auf die föderalistische Struktur Osterreichs, Schriftenreihe des Instituts für Föderalismusforschung, Band 47, hrsg. von Peter Pernthaler, Wien 1989, S. $71 \mathrm{ff}$. (Zitierweise: = Landesparlamente=)

Rudolf, Walter: Bundesländer und Europäisches Gemeinschaftsrecht, in: Staatsrecht, Völkerrecht, Europarecht, hrsg. von Ingo von Münch, Berlin, New York 1981, S. 117ff. (Zitierweise: = Bundesländer und Gemeinschaftsrecht $=$ )

Sachverständigenrat zur Begutachtung der gesamtwirtschaftlichen Entwicklung: Chancen für einen langen Aufschwung, Jahresgutachten 1984/85, Stuttgart, Mainz 1984. (Zitierweise: = Jahresgutachten $1984 / 85^{\prime}=$ )

Sachverständigenrat zur Begutachtung der gesamtwirtschaftlichen Entwicklung: Weichenstellungen für die neunziger Jahre, Jahresgutachten 1989/90, Stuttgart 1989. (Zitierweise: = Jahresgutachten 1989/90=)

Sachverständigenrat zur Begutachtung der gesamtwirtschaftlichen Entwicklung: Auf dem Wege zur deutschen Einheit, Jahresgutachten 1990/91, Stuttgart 1990. (Zitierweise: = Jahresgutachten 1990/91=)

Schäfers, Manfred; Starbatty, Joachim: Das Instrumentarium der EG zur Förderung innergemeinschaftlicher Kohäsion, in: Aus Politik und Zeitgeschichte, Beilage zur Wochenschrift "Das Parlament", B 28/90 vom 6.7.1990, S. 3ff. (Zitierweise: =Kohäsion=)

Scharpf, Fritz W.: Die Politikverflechtungs-Falle. Europäische Integration und deutscher Föderalismus im Vergleich, in: Politische Vierteljahresschrift, Zeitschrift der Deutschen Vereinigung für Politische Wissenschaft, 26. Jg. (1985), S. 323ff. (Zitierweise: = Politikverflechtungs-Falle=)

Scheele, Martin: Sektorale Einkommensübertragungen im Rahmen der Agrarsozialpolitik, in: Agrarwirtschaft, 38. Jg. (1989), S. 203ff. (Zitierweise: $=$ Sektorale Einkommensübertragungen $=$ )

Scheer, Christian; Vogelbusch, Friedrich: Gains and Losses of European Community Membership: The Case of the Federal Republik of Germany. Fiscal Policy. Bonn 1989. (Zitierweise: = Gains and Losses=)

Scheide, Joachim; Trapp, Peter: Etatdisziplin vom Markt. Auch in einer Währungsunion müssen die beteiligten Länder nicht auf eine nationale Finanzpolitik verzichten, in: Frankfurter Allgemeine Zeitung vom 23.2.1991, S. 13. (Zitierweise: =Etatdisziplin=)

Schemmel, Lothar: Steuerharmonisierung in der Europäischen Gemeinschaft, hrsg. vom Karl-Bräuer-Institut des Bundes der Steuerzahler, Bonn 1989. (Zitierweise: $=$ Steuerharmonisierung $=$ ) 
Scheper, Wilhelm: Perspektiven für eine Weiterentwicklung der Gemeinsamen Agrarpolitik, in: Landwirtschaft, Umwelt und ländlicher Raum. Herausforderungen an Europa, Hermann Priebe zum 80. Geburtstag, hrsg. von Winfried Urff und Hermann von Meyer, Baden-Baden 1987, S. 105ff. (Zitierweise: = Gemeinsame Agrarpolitik=)

Schmidhuber, Peter M.: Chancen und Risiken der Europäischen Wirtschaftsund Währungsunion, Rede in der Bayerischen Landesvertretung in Bonn am 30.5.1990, abgedruckt in: Auszüge aus Presseartikeln, hrsg. von der Deutschen Bundesbank, Nr. 45 vom 5.6.1990, S. $15 \mathrm{ff}$.

Schmidhuber, Peter M.: Der Binnenmarkt 1992. Eine Herausforderung für die Gesetzgebung der Gemeinschaft und der Mitgliedstaaten, in: EuropaArchiv, Folge 2 (Beiträge und Berichte), 44. Jg. (1989), S. $75 \mathrm{ff}$. (Zitierweise: = Gesetzgebung $=$ )

Schmidt, Kurt: Grundprobleme der Besteuerung, in: Handbuch der Finanzwissenschaft, hrsg. von Fritz Neumark unter der Mitarbeit von Norbert Andel und Heinz Haller, Band II, 3., gänzlich neubearbeitete Auflage, Tübingen 1980, S. 119ff. (Zitierweise: =Grundprobleme=)

Schrader, Jörg-Volker: EG-Gipfelbeschlüsse zur Agrar- und Haushaltspolitik. Schritte in die falsche Richtung, Kieler Diskussionsbeiträge Nr. 143, Kiel 1988. (Zitierweise: =Agrar- und Haushaltspolitik=)

Schüler, Manfred: Die vierte Ebene. Haushalts- und finanzpolitische Probleme der Europäischen Gemeinschaften, in: Europa-Archiv, Folge 2 (Beiträge und Berichte), Jg. 29 (1974), S. 45ff. (Zitierweise: =Die vierte Ebene=)

Schweitzer, Michael: Bundesstaat und Europäische Gemeinschaft, in: Auswirkungen eines EG-Beitritts auf die föderalistische Struktur Osterreichs, Schriftenreihe des Instituts für Föderalismus, Band 47, hrsg. von Peter Pernthaler, Wien 1989, S. 15ff. (Zitierweise: = Bundesstaat und EG=)

Seidenfus, Hellmuth: Der europäische Verkehrsmarkt nach 1992, in: Wirtschaftsdienst, 68. Jg. (1988), S. $622 \mathrm{ff}$.

Selmer Peter: Die Anleihekompetenzen der Europäischen Gemeinschaften, in: Studien zum internationalen Wirtschaftsrecht und Atomrecht, hrsg. von K.-H. Böckstiegel et al., Köln u. a. 1984, S. 24ff. (Zitierweise: = Anleihekompetenzen $=$ )

Senf, Paul: Kurzfristige Haushaltsplanung, in: Handbuch der Finanzwissenschaft, hrsg. von Fritz Neumark unter Mitarbeit von Norbert Andel und Heinz Haller, Band I, 3., gänzlich neubearbeitete Auflage, Tübingen 1977, S. $371 \mathrm{ff}$. (Zitierweise: =Kurzfristige Haushaltsplanung =)

Siebert, Horst: Regional Aspects of Environmental Allocation, in: Zeitschrift für die gesamte Staatswissenschaft, Band 131 (1975), S. 496ff. (Zitierweise: =Environmental Allocation=)

Spahn, P. B.: Die Zukunft der Finanzen der Europäischen Gemeinschaften, in: Agrarwirtschaft, Zeitschrift für Betriebswirtschaft, Marktforschung und Agrarpolitik, Jg. 36 (1987), S. 1ff. (Zitierweise: =Zukunft der Finanzen $=$ ) 
Stahl, Gerhard: Mittelfristige Finanzplanung - eine Antwort auf EG-Haushaltsrisiken?, in: Wirtschaftsdienst, 68. Jg. (1988), S. 419ff. (Zitierweise: $=$ Mittelfristige Finanzplanung $=$ )

Starbatty, Joachim; Vetterlein, Uwe: Die Technologiepolitik der Europäischen Gemeinschaft, Entstehung, Praxis und ordnungspolitische Konformität, Baden-Baden 1990. (Zitierweise: = Technologiepolitik=)

Starbatty, Joachim; Vetterlein, Uwe: Technologiepolitische Aufgabenteilung in der Europäischen Gemeinschaft, in: Wirtschaftsdienst, 70. Jg. (1990), S. 96ff. (Zitierweise: =Technologiepolitische Aufgabenteilung =)

Statistisches Bundesamt: Volkswirtschaftliche Gesamtrechnungen, Fachserie 18, Reihe S.8, Revidierte Ergebnisse 1960 bis 1984, Mainz 1985. (Zitierweise: = Revidierte Ergebnisse =)

Statistisches Bundesamt: Statistisches Jahrbuch für das Ausland 1989, Stuttgart 1989. (Zitierweise: =Ausland 1989=)

Statistisches Bundesamt: Statistisches Jahrbuch für das Ausland 1990, Stuttgart 1990. (Zitierweise: = Ausland 1990=)

Statistisches Bundesamt: Volkswirtschaftliche Gesamtrechnungen, Fachserie 18. Reihe 1.3, Konten und Standardtabellen, Hauptbericht 1989, Mainz 1990. (Zitierweise: $=$ Konten und Standardtabellen $=$ )

Stavenhagen, Lutz: Chancen und Herausforderungen eines europäischen Verkehrsmarktes, in: Bulletin, hrsg. vom Presse- und Informationsamt der Bundesregierung, Nr. 153 vom 15.11.1988.

Steinle, Wolfgang J.: Europäische Regionalpolitik zwischen Mittelkonzentration, Koordination und Flexibilität, in: Raumordnung und Raumforschung, 41. Jg. (1983), S. $3 \mathrm{ff}$.

Stoiber, Edmund: Auswirkungen der Entwicklung Europas zur Rechtsgemeinschaft auf die Länder der Bundesrepublik Deutschland, in: Europa-Archiv, Folge 2 (Beiträge und Berichte), 42. Jg. (1987), S. 543ff. (Zitierweise: $=$ Rechtsgemeinschaft $=$ )

Stoll, Karin: Alternativen der EG-Finanzierung, in: Europa finanzieren - ein föderalistisches Modell, hrsg. von der Bertelsmann-Stiftung, Gütersloh 1990, S. 143ff. (Zitierweise: = Alternativen der EG-Finanzierung=)

Strasser, Daniel: Die Finanzen Europas, 2. Auflage, Luxemburg 1982, (Zitierweise: =Finanzen Europas $=$ )

Theiler, Jörg: Föderalismus - Voraussetzung oder Ergebnis rationaler Politik? Zur ökonomisch optimalen Struktur kollektiver Entscheidungsverfahren, Bern u. a. 1977. (Zitierweise: =Föderalismus=)

Thoroe, Carsten S.: Die europäische Agrarpolitik als Finanzausgleichsproblem, in: Probleme des Finanzausgleichs III, Finanzausgleich im Rahmen der Europäischen Gemeinschaften, hrsg. von Dieter Pohmer, Schriften des Vereins fuir Socialpolitik, N. F., Band 96/III, Berlin 1981, S. 85ff. (Zitierweise: =Europäische Agrarpolitik=) 
Thoroe, Carsten S.: Die Zucker- und Milchabgaben als EG-Produktionssteuer, in: Finanzarchiv, N. F., Band 42 (1984), S. 350ff. (Zitierweise: =EG-Produktionssteuern $=$ )

Tiebout, Charles: A Pure Theory of Local Expenditures, in: Journal of Political Economy, Vol. 64 (1956), S. $416 \mathrm{ff}$. (Zitierweise: =Local Expenditures $=$ )

Tiebout, Charles: Eine ökonomische Theorie fiskalischer Dezentralisierung, in: Föderalismus, hrsg. von Guy Kirsch, Stuttgart, New York 1977, S. 36ff. (Zitierweise: = Fiskalische Dezentralisierung $=$ )

Tietmeyer, Hans: Voraussetzungen eines Europäischen Zentralbankensystems, in: Außenwirtschaft, 45. Jg. (1990), S. $301 \mathrm{ff}$. (Zitierweise: = Voraussetzungen $=$ )

Timmann, Hans-Jörg: Die jüngste Finanzreform (1988) und ihre Umsetzung, in: Reform der EG-Finanzierung, hrsg. von Dieter Biehl und Gero Pfennig, Bonn 1990, S. 25ff. (Zitierweise: =Finanzreform=)

Tullock, Gordon: Föderalismus. Zum Problem der optimalen Kollektivgröße, in: Föderalismus, hrsg. von Guy Kirsch, Stuttgart, New York 1977, S. 27ff. (Zitierweise: $=$ Föderalismus $=$ )

Ungar, Peter: Vorschläge für eine neue EG-Finanzverfassung auf dem Prüfstand. Bericht vom Symposium "Eine neue Finanzverfassung für die EG" des Europa-Zentrums Bonn, in: Integration, Band 13 (1990), S. $126 \mathrm{ff}$. (Zitierweise: $=$ EG-Finanzverfassung $=)$

Vertrag zu Einsetzung eines gemeinsamen Rates und einer gemeinsamen Kommission der Europäischen Gemeinschaften vom 8.4.1965, in: Bundesgesetzblatt II, S. 1454ff.

Graf Vitzthum, Wolfgang: Der Föderalismus in der europäischen und internationalen Einbindung der Staaten, in: Archiv des öffentlichen Rechts, Band 115 (1990), S. 281ff. (Zitierweise: = Föderalismus=)

Walter, Ingo: International Economics of Pollution, New York, Toronto 1975. (Zitierweise: $=$ Pollution $=$ )

Weidner, Helmut; Knoepfel, Peter: Implementierungschancen der EG-Richtlinie zur $\mathrm{SO}_{2}$-Luftreinhaltepolitik. Ein kritischer Beitrag zur Internationalisierung von Umweltpolitik, in: Zeitschrift für Umweltpolitik, Jg. 4 (1981), S. 27ff. (Zitierweise: =Implementierungschancen $=$ )

Weinstock, Ulrich: Nur eine europäische Umwelt, in: Zeitschrift für Umweltpolitik, Jg. 6 (1983), S. 1ff. (Zitierweise: =Europäische Umwelt=)

Wessels, Wolfgang: Die deutschen Länder in der EG-Politik: Selbstblockierung oder pluralistische Dynamik, in: Die deutschen Länder und die Europäischen Gemeinschaften, hrsg. von Rudolf Hrbek und Uwe Thaysen, Baden-Baden 1986, S. $181 \mathrm{ff}$. (Zitierweise: = Deutsche Länder in der EGPolitik=) 
Willgerodt, Hans: Die Agrarpolitik der Europäischen Gemeinschaft in der Krise, in: Ordo, Band 34 (1983), S. $97 \mathrm{ff}$. (Zitierweise: = Agrarpolitik der $\mathrm{EG}=$ )

Williams, Alan: The Provision of Public Goods in a System of Local Government, in: Journal of Political Economy, Vol. 74 (1966), S. 18ff. (Zitierweise: $=$ Public Goods $=$ )

Willms, Manfred: Der Delors-Plan und die Anforderungen an eine gemeinsame Europäische Geldpolitik, in: Kredit und Kapital, Jg. 23 (1990), S. 30ff.

Willms, Manfred: Grundprobleme eines Europäischen Zentralbankstatuts, in: Wirtschaftsdienst, 70. Jg. (1990), S. 551ff. (Zitierweise: =Grundproble$\mathrm{me}=$ )

Wissenschaftlicher Beirat beim Bundesministerium der Finanzen: Gutachten zur Aussagefähigkeit staatswirtschaftlicher Quoten, abgedruckt in: Bulletin, hrsg. vom Presse- und Informationsamt der Bundesregierung, Nr. 90 vom 30.7.1976, S. 849 ff. (Zitierweise: = Aussagefähigkeit staatswirtschaftlicher Quoten=)

Wissenschaftlicher Beirat beim Bundesministerium für Wirtschaft: Stellungnahme zum Delors-Bericht vom 5.6.1989, Brief an den Bundesminister für Wirtschaft vom 8.6.1989. (Zitierweise: =Stellungnahme=)

Wissenschaftlicher Dienst des deutschen Bundestages: Die Harmonisierung der Mehrwert- und Verbrauchsteuern im Hinblick auf den europäischen Binnenmarkt, Materialien Nr. 106, Bonn 1989. (Zitierweise: = Harmonisierung =)

Wust, Herbert F.: Föderalismus. Grundlage für Effizienz in der Staatswirtschaft, Göttingen 1981. (Zitierweise: = Föderalismus=)

Zimmermann, Horst: EG-Begrenzung für die deutsche Regionalpolitik. Ansätze und mögliche Konsequenzen einer EG-Konvergenzpolitik, in: Wirtschaftsdienst, 66. Jg. (1986), S. 92ff. (Zitierweise: =EG-Begrenzung für deutsche Regionalpolitik=)

Zimmermann, Horst: Gewichtsverlagerung im föderativen Staatsaufbau unter EG-Einfluß3?, in: Wirtschaftsdienst, Jg. 70 (1990), S. 451-456. (Zitierweise: = Gewichtsverlagerung $=$ )

Zimmermann, Horst; Henke, Klaus-Dirk: Finanzwissenschaft. Eine Einführung in die Lehre von der öffentlichen Finanzwisssenschaft, 5., überarbeitete und ergänzte Auflage, München 1987. (Zitierweise: =Finanzwissenschaft =) 


\section{FINANZWISSENSCHAFTLICHE SCHRIFTEN}

Band 1 Werner Steden: Finanzpolitik und Einkommensverteilung. Ein Wachstums- und Konjunkturmodell der Bundesrepublik Deutschland. 1979.

Band 2 Rainer Hagemann: Kommunale Finanzplanung im föderativen Staat. 1976.

Band 3 Klaus Scherer: Maßstäbe zur Beurteilung von konjunkturellen Wirkungen des öffentlichen Haushalts. 1977.

Band 4 Brita Steinbach: "Formula Flexibility" - Kritische Analyse und Vergleich mit diskretionärer Konjunkturpolitik. 1977.

Band 5 Hans-Georg Petersen: Personelle Einkommensbesteuerung und Inflation. Eine theoretisch-empirische Analyse der Lohn- und veranlagten Einkommensteuer in der Bundesrepublik Deutschland. 1977.

Band 6 Friedemann Tetsch: Raumwirkungen des Finanzsystems der Bundesrepublik Deutschland. Eine Untersuchung der Auswirkungen der Finanzreform von 1969 auf die Einnahmenposition der untergeordneten Gebietskörperschaften und ihrer regionalpolitischen Zieladäquanz. 1978.

Band 7 Wilhelm Pfähler: Normative Theorie der fiskalischen Besteuerung. Ein methodologischer und theoretischer Beitrag zur Integration der normativen Besteuerungstheorie in der Wohlfahrtstheorie. 1978.

Band 8 Wolfgang Wiegard: Optimale Schattenpreise und Produktionsprogramme für öffentliche Unternehmen. Second-Best Modelle im finanzwirtschaftlichen Staatsbereich. 1978.

Band 9 Hans P. Fischer: Die Finanzierung des Umweltschutzes im Rahmen einer rationalen Umweltpolitik. 1978.

Band 10 Rainer Paulenz: Der Einsatz finanzpolitischer Instrumente in der Forschungs- und Entwicklungspolitik. 1978.

Band 11 Hans-Joachim Hauser: Verteilungswirkungen der Staatsverschuldung. Eine kreislauftheoretische Inzidenzbetrachtung. 1979.

Band 12 Gunnar Schwarting: Kommunale Investitionen. Theoretische und empirische Untersuchungen der Bestimmungsgründe kommunaler Investitionstätigkeit in NordrheinWestfalen 1965-1972. 1979.

Band 13 Hans-Joachim Conrad: Stadt-Umland-Wanderung und Finanzwirtschaft der Kernstädte. Amerikanische Erfahrungen, grundsätzliche Zusammenhänge und eine Fallstudie für das Ballungsgebiet Frankfurt am Main. 1980.

Band 14 Cay Folkers: Vermögensverteilung und staatliche Aktivität. Zur Theorie distributiver Prozesse im Interventionsstaat. 1981.

Band 15 Helmut Fischer: US-amerikanische Exportförderung durch die DISC-Gesetzgebung. 1981.

Band 16 Günter Ott: Einkommensumverteilungen in der gesetzlichen Krankenversicherung. Eine quantitative Analyse. 1981.

Band 17 Johann Hermann von Oehsen: Optimale Besteuerung. (Optimal Taxation). 1982.

Band 18 Richard Kössler: Sozialversicherungsprinzip und Staatszuschüsse in der gesetzlichen Rentenversicherung. 1982.

Band 19 Hinrich Steffen: Zum Handlungs- und Entscheidungsspielraum der kommunalen Investitionspolitik in der Bundesrepublik Deutschland. 1983.

Band 20 Manfred Scheuer: Wirkungen einer Auslandsverschuldung des Staates bei flexiblen Wechselkursen. 1983. 
Band 21 Christian Schiller: Staatsausgaben und crowding-out-Effekte. Zur Effizienz einer Finanzpolitik keynesianischer Provenienz. 1983.

Band 22 Hannelore Weck: Schattenwirtschaft: Eine Möglichkeit zur Einschränkung der öffentlichen Verwaltung? Eine ökonomische Analyse. 1983.

Band 23 Wolfgang Schmitt: Steuern als Mittel der Einkommenspolitik. Eine Ergänzung der Stabilitätspolitik? 1984.

Band 24 Wolfgang Laux: Erhöhung staatswirtschaftlicher Effizienz durch budgetäre Selbstbeschränkung? Zur Idee einer verfassungsmäßig verankerten Ausgabengrenze. 1984.

Band 25 Brita Steinbach-van der Veen: Steuerinzidenz. Methodologische Grundlagen und empirisch-statistische Probleme von Länderstudien. 1985.

Band 26 Albert Peters: Okonomische Kriterien für eine Aufgabenverteilung in der Marktwirtschaft. Eine deskriptive und normative Betrachtung für den Allokationsbereich. 1985.

Band 27 Achim Zeidler: Möglichkeiten zur Fortsetzung der Gemeindefinanzreform. Eine theoretische und empirische Analyse. 1985.

Band 28 Peter Bartsch: Zur Theorie der längerfristigen Wirkungen 'expansiver' Fiskalpolitik. Eine dynamische Analyse unter besonderer Berücksichtigung der staatlichen Budgetbeschränkung und ausgewählter Möglichkeiten der öffentlichen Defizitfinanzierung. 1986.

Band 29 Konrad Beiwinkel: Wehrgerechtigkeit als finanzpolitisches Verteilungsproblem. Möglichkeiten einer Kompensation von Wehrungerechtigkeit durch monetäre Transfers. 1986.

Band 30 Wolfgang Kitterer: Effizienz- und Verteilungswirkungen des Steuersystems. 1986.

Band 31 Heinz Dieter Hessler: Theorie und Politik der Personalsteuern. Eine Kritik ihrer Einkommens- und Vermögensbegriffe. 1987.

Band 32 Wolfgang Scherf: Die beschättigungspolitische und fiskalische Problematik der Arbeitgeberbeiträge zur Rentenversicherung. Eine Auseinandersetzung mit der Kritik an der lohnbezogenen Beitragsbemessung. 1987.

Band 33 Andreas Măstle: Die Steuerunion. Probleme der Harmonisierung spezifischer Gütersteuern. 1987.

Band 34 Günter Ott: Internationale Verteilungswirkungen im Finanzausgleich der Europäischen Gemeinschaften. 1987.

Band 35 Heinz Haller: Zur Frage der zweckmäßigen Gestalt gemeindlicher Steuern. Ein Diskussionsbeitrag zur Gemeindesteuerreform. 1987.

Band 36 Thomas Kuhn: Schlüsselzuweisungen und fiskalische Ungleichheit. Eine theoretische Analyse der Verteilung von Schlüsselzuweisungen an Kommunen. 1988.

Band 37 Walter Hahn: Steuerpolitische Willensbildungsprozesse in der Europäischen Gemeinschaft. Das Beispiel der Umsatzssteuer-Harmonisierung. 1988.

Band 38 Ulrike Hardt: Kommunale Finanzkraft. Die Problematik einer objektiven Bestimmung kommunaler Einnahmemöglichkeiten in der gemeindlichen Haushaltsplanung und im kommunalen Finanzausgleich. 1988.

Band 39 Jochen Michaelis: Optimale Finanzpolitik im Modell überlappender Generationen. 1989.

Band 40 Bernd Raffelhüschen: Anreizwirkungen der sozialen Alterssicherung. Eine dynamische Simulationsanalyse. 1989.

Band 41 Berend Diekmann: Die Anleihe- und Darlehenstransaktionen der Europäischen Gemeinschaften. 1990.

Band 42 Helmut Kaiser: Konsumnachfrage, Arbeitsangebot und optimale Haushaltsbesteuerung. Theoretische Ergebnisse und mikroökonometrische Simulation für die Bundesrepublik Deutschland. 1990. 
Band 43 Rüdiger von Kleist: Das Gramm-Rudman-Hollings-Gesetz. Ein gescheiterter Versuch der Haushaltskonsolidierung. 1991.

Band 44 Rolf Hagedorn: Steuerhinterziehung und Finanzpolitik. Ein theoretischer Beitrag unter besonderer Berücksichtigung der Hinterziehung von Zinserträgen. 1991.

Band 45 Cornelia S. Behrens: Intertemporale Verteilungswirkungen in der gesetzlichen Krankenversicherung der Bundesrepublik Deutschland. 1991.

Band 46 Peter Saile: Ein ökonomischer Ansatz der Theorie der intermediären Finanzgewalten Die Kirchen als Parafisci. 1992.

Band 47 Peter Gottfried: Die verdeckten Effizienzwirkungen der Umsatzsteuer. Eine empirische allgemeine Gleichgewichtsanalyse. 1992.

Band 48 Andreas Burger: Umweltorientierte Beschäftigungsprogramme. Eine Effizienzanalyse am Beispiel des "Sondervermögens Arbeit und Umwelt". 1992.

Band 49 Jeanette Malchow: Die Zuordnung verteilungspolitischer Kompetenzen in der Europäischen Gemeinschaft. Eine Untersuchung aufgrund einer Fortentwicklung der ökonomischen Theorie des Föderalismus. 1992.

Band 50 Barbara Seidel: Die Einbindung der Bundesrepublik Deutschland in die Europäischen Gemeinschaften als Problem des Finanzausgleichs. 1992.

Band 51 Ralph Wiechers: Markt und Macht im Rundfunk. Zur Stellung der offentlich-rechtlichen Rundfunkanstalten im dualen Rundfunksystem der Bundesrepublik Deutschland. 1992. 


\section{Günter Ott}

\section{Internationale Verteilungswirkungen im Finanzausgleich der Europäischen Gemeinschaften}

Frankfurt/M., Bern, New York, Paris, 1987. VI. 323 S.

Finanzwissenschaftliche Schriften. Bd. 34

ISBN 3-8204-0196-2

br. DM 80.--

Ein seit langem ungelöstes Problem der EG ist eine allgemein akzeptierte Verteilung der Finanzierungslasten und Ausgaben auf die Mitgliedsstaaten. Dabei sind die Fragen nach den Größenordnungen der Distributionswirkungen sowie nach einem normativen Rahmen immer wieder von aktuellem politischen Interesse. Die vorliegende Studie versucht nun, zum einen genauere und umfassende empirische Ergebisse als bisher zu liefern. Zum anderen werden diese Verteilungswirkungen anhand der für die Finanzwirtschaft der Gemeinschaft ableitbaren Zielsetzungen und postulierbaren normativen Maßstäbe beurteilt und wird die Richtung möglicher Korrekturen aufgezeigt.

Aus dem Inhalt: Theoretisch-methodische Grundfragen der Inzidenzanalyse - Empirische Analyse der zwischenstaatlichen Verteilungswirkungen - Normative Ansätze zur Beurteilung der Verteilungswirkungen

"Wer sich über den europäischen Finanzausgleich, über die Töpfchenwirtschaft der europäischen Institutionen ein Bild machen will, findet in der OTTschen Arbeit reichhaltiges Anschauungsmaterial."

Gerold Krause-Junk in Finanzarchiv

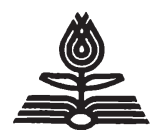

Verlag Peter Lang Frankfurt a.M. Berlin · Bern · New York • Paris • Wien Auslieferung: Verlag Peter Lang AG, Jupiterstr. 15, CH-3000 Bern 15 Telefon (004131) 9411122, Telefax (004131) 9411131 
Barbara Pflugmann-Hohlstein - 978-3-631-75187-9 Downloaded from PubFactory at 01/11/2019 07:20:05AM

via free access 